\title{
MEASUREMENT OF THE STRANGE QUARK CONTRIBUTION TO PROTON
} STRUCTURE THROUGH PARITY VIOLATING ELECTRON-PROTON SCATTERING

\author{
BY \\ KAZUTAKA NAKAHARA \\ Bachelor of Science, Carnegie-Mellon University, 2000
}

\section{DISSERTATION}

Submitted in partial fulfillment of the requirements for the degree of Doctor of Philosophy in Physics in the Graduate College of the University of Illinois at Urbana-Champaign, 2006

Urbana, Illinois 


\section{Abstract}

The $G^{0}$ (G-Zero) forward angle experiment completed in Hall $\mathrm{C}$ of the Thomas Jefferson National Accelerator Facility (TJNAF) has measured the parity violating asymmetries in elastic electronproton scattering over a $Q^{2}$ range of $0.12<Q^{2}<1.0(\mathrm{GeV} / \mathrm{c})^{2}$. A linear combination of the strange electric $\left(G_{E}^{s}\right)$ and magnetic $\left(G_{M}^{s}\right)$ form factors calculated from these asymmetries indicate a nonzero contribution of the strange quark to the charge and magnetization structure of the proton in the above kinematic range at a $89 \%$ confidence level. The results show a previously unmeasured $Q^{2}$ dependence of the strange form factors. Combining the $G^{0}$ results with previous parity violating experiments show that at $Q^{2}=0.1(\mathrm{GeV} / \mathrm{c})^{2}$

$$
\begin{gathered}
G_{M}^{s}=0.62 \pm 0.31 \\
G_{E}^{s}=-0.013 \pm 0.028
\end{gathered}
$$

At intermediate $Q^{2}$ of about $0.23(G e V / c)^{2}$, a consistent value of $G_{M}^{s}$ is seen compared to previous experiments, together with a measurement that may imply a negative value of $G_{E}^{s}$. For

$Q^{2}$ above $0.5(\mathrm{GeV} / \mathrm{c})^{2}$ a consistently positive value for the linear combination of the strange form factors is seen. 


\section{Acknowledgments}

It has been a great pleasure to work with those in the $G^{0}$ collaboration as well as the staff members at the Thomas Jefferson National Accelerator Facility. There are countless people who have helped me over the past few years, and I write this fully aware that a short acknowledgement would not do justice.

I would like to thank my advisor, Doug Beck, for the guidance, support, and friendship throughout my graduate career. Perhaps the greatest experience I take away from my graduate career is the opportunity I had to see physics done at many different levels, from a smaller university setting to that at a large national laboratory. I appreciate Doug's efforts and advice in allowing me to pursue this rich experience.

I would like to acknowledge Steve Williamson, Damon Spayde, and Andy Kenyon who have guided me through the testing and commissioning of the $G^{0}$ magnet here at Illinois as well as at JLab. Mark Pitt has given me great advice during the development of the $G^{0}$ beam, and the subsequent maintenance of its stability. His insight and daily advice during that process is greatly appreciated. Retief Neveling has made the source studies between the first and second commissioning runs truly enjoyable. Betsy Beise, Dave Armstrong, Serge Kox, Jacques Arvieux, and countless others have given great advice to the postdocs and graduate students throughout the course of the experiment, and were essential in guiding the experiment to a successful conclusion. I'm sure I will never forget the sound of the "whip" that Serge would unleash on us to analyze the data.

Julie Roche, Paul King, Damon Spayde, Dave Gaskell, as well as countless other postdocs and faculty were instrumental in guiding the analysis of the $G^{0}$ data, and keeping the experiment going during the run.

I have had the privilege to work with many $G^{0}$ graduate students (Guillaume Batigne, Silviu 
Covrig, Benoit Guillon, Lars Hannelius, Jianglai Liu, Sarah Phillips, Tanya Horn, and many others) from many different backgrounds. In particular, I would like to thank Jianglai ("it's a GOAT!") Liu for all his help involving various projects, and giving me a better understanding of the experiment. I hope he will never forget the loud clicking sounds that came from two cubicles over while he was at the counting house. I enjoyed the times (and the near-daily tea breaks) I spent with Silviu while he was writing his thesis. His philosophical insight into various matters of life were truely fascinating. Lars and Benoit have been instrumental in understanding the background with their systematic studies involving simulations. Sarah, with whom I have a standing bet to take over the world, has always made the experiment enjoyable. The times we spent travelling in Colorado (with Itaru and Sean) were alot of fun. I would like to thank Tanya for taking countless shifts for G0 while she was herself working on her own thesis experiment. My only regret is that I was, in the end, not able to get her to take a sip of coffee.

I extend my thanks to the Hall $\mathrm{C}$ and Accelerator personnel for assisting and actively contributing in the successful $G^{0}$ run. The beam development at JLab involved extensive effort by the accelerator group. I would like to thank Matt Poelker, Joe Grames, and the source group for their continuous effort in assuring delivery of the beam. Members of CASA, including Yu-Chiu Chao, Lia Merminga, Reza Kazimi, Swapan Chattopadhyay, Andrew Hutton, and the entire accelerator group worked tirelessly to improve and maintain beam quality, and it has been an enlightening experience to work with them.

Finally, my parents have, from the time I was little, stressed the importance of education, and I am grateful for their support throughout my years in school and for encouraging me to pursue my dreams.

This work is supported in part by CNRS (France), DOE (U.S.), NSERC (Canada), and NSF (U.S.). 


\section{Table of Contents}

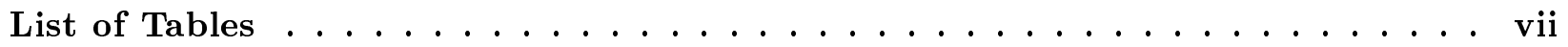

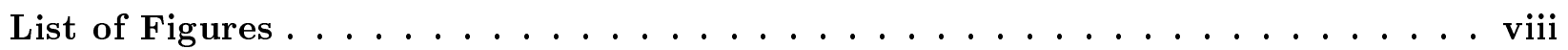

Chapter 1 Introduction ........................ 1

1.1 The Strange Sea . . . . . . . . . . . . . . . . . . . 2

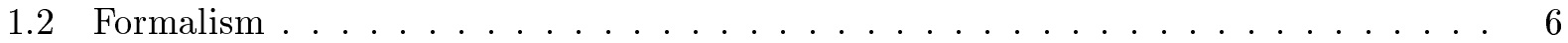

1.2.1 Parity-Violating Asymmetry . . . . . . . . . . . . . . . . 13

1.2.2 Electroweak Radiative Corrections . . . . . . . . . . . . . . . . . . . 14

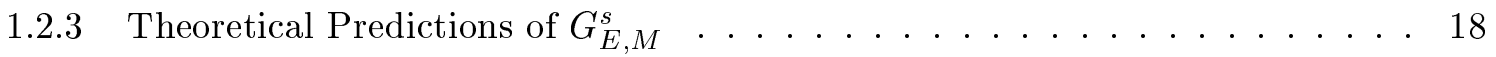

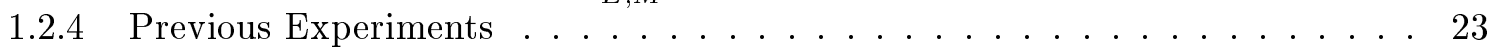

Chapter 2 G0 Experimental Apparatus . . . . . . . . . . . . . 30

2.1 The $G^{0}$ Polarized Electron Beam . . . . . . . . . . . . . . . . . . . 32

$2.2 G^{0}$ Beam Properties $\ldots \ldots \ldots \ldots \ldots \ldots$

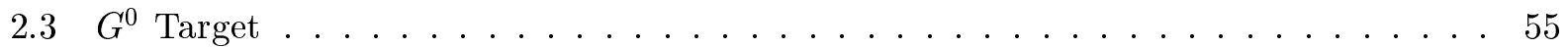

2.3.1 Target Systematics . . . . . . . . . . . . . . . . . . 59

$2.4 G^{0}$ Superconducting Magnet System . . . . . . . . . . . . . . . . . . . . 61

$2.5 G^{0} \mathrm{FPD}$ and Electronics . . . . . . . . . . . . . . . . 67

Chapter 3 Analysis ......................... 74

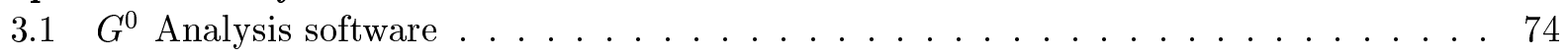

3.1 .1 ROOT files and the Database . . . . . . . . . . . . . . 74

3.1.2 Data Selection . . . . . . . . . . . . . . . . 75

3.2 Raw Proton Asymmetry . . . . . . . . . . . . . . . . . . 76

3.3 G0GEANT simulation . . . . . . . . . . . . . . . . 80

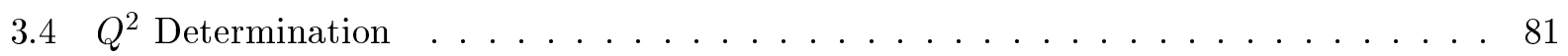

3.5 Leakage correction . . . . . . . . . . . . . . . . . . . . . . 85

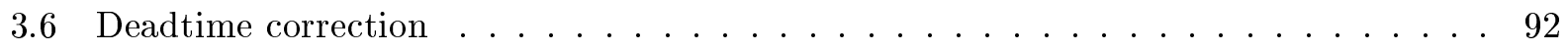

3.7 Linear Regression correction . . . . . . . . . . . . . . . . . . . . 96

3.8 Background correction . . . . . . . . . . . . . . . . . . 101

3.9 Polarization correction . . . . . . . . . . . . . . . . . . . 125

3.10 Radiative corrections . . . . . . . . . . . . . . . . . . . 130

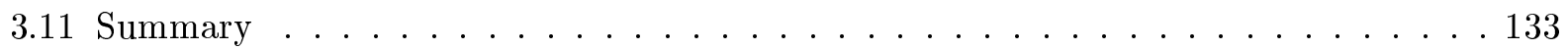

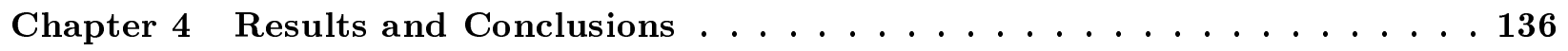




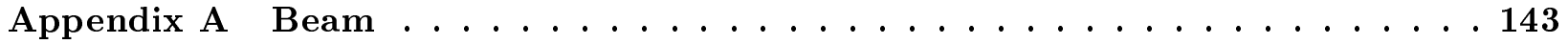

A.1 Lasertable Studies . . . . . . . . . . . . . . . . . . . . . . . . . . 143

A.2 Beam Orthogonality . . . . . . . . . . . . . . . . . . . . 144

A.3 Quadrupole Adjustments . . . . . . . . . . . . . . . . . 146

A.4 Beam Correlations . . . . . . . . . . . . . . . . . . . . 148

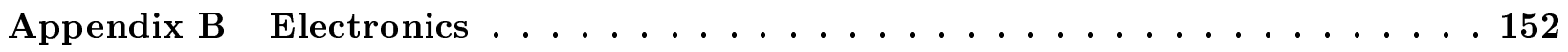

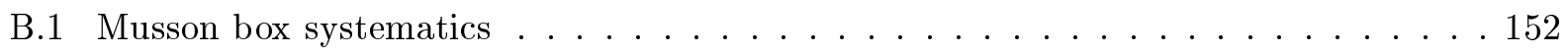

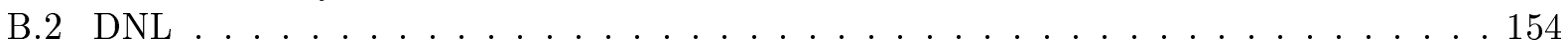

B.3 Electronics Efficiency . . . . . . . . . . . . . . . . . 155

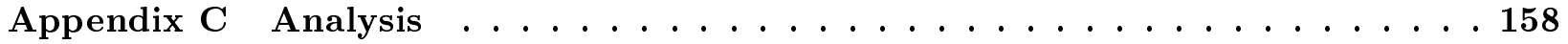

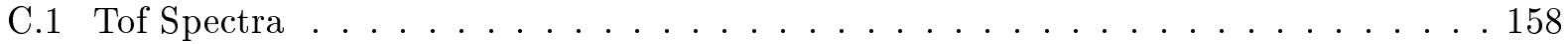

C.2 Linear Regression Slopes . . . . . . . . . . . . . . . . . . 167

C.3 Systematics . . . . . . . . . . . . . . . . . . . 169

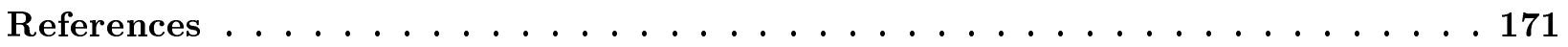

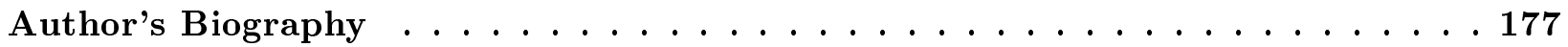




\section{List of Tables}

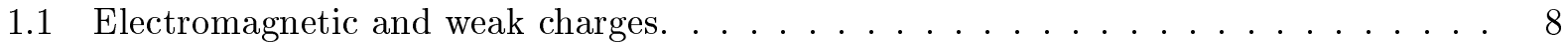

1.2 Values of vector and axial weak charges. . . . . . . . . . . . . . . 17

1.3 Terms involved in electroweak radiative corrections. . . . . . . . . . . . . . . 18

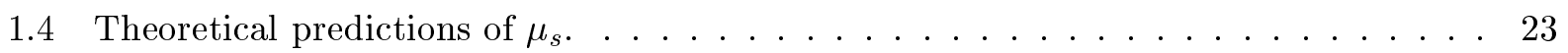

2.1 Resolutions of HC beam parameters seen in the experiment. . . . . . . . . . . . . 43

2.2 Helicity-correlated beam parameters achieved during the $G^{0}$ forward angle produc-

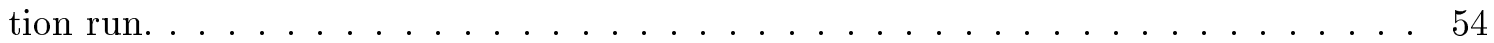

3.1 Rates and yields observed due to leakage and $G^{0}$ beams for the elastic cut and cut0. 88

3.2 Total false asymmetries calculated from multiple linear regression. . . . . . . . . . 102

3.3 Reduced $\chi^{2}$ from the asymmetry fits for detectors $1-14$ a . . . . . . . . . . . . 115

3.4 Contributions to the the systematic uncertainties for detectors 1-14a. . . . . . . . 118

3.5 Reduced $\chi^{2}$ for the two-step fitting technique and the detector-interpolation technique. The $\chi^{2}$ are for all 8 octants combined. . . . . . . . . . . . . . . 121

3.6 Møller systematics. . . . . . . . . . . . . . . . . . . . . . . . . 128

3.7 Measured and corrected asymmetries. . . . . . . . . . . . . . 135

4.1 Final results of the $G^{0}$ forward angle measurement. . . . . . . . . . . . . . . . 137

C.1 IHWP OUT linear regression slopes for the transverse positions and angles averaged over all octants. . . . . . . . . . . . . . . . . . . 167

C.2 IHWP OUT linear regression slopes for energy and beam current averaged over all

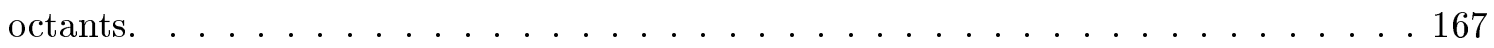

C.3 IHWP IN linear regression slopes for the transverse positions and angles averaged over all octants. . . . . . . . . . . . . . . . . . . 168

C.4 IHWP IN linear regression slopes for energy and beam current averaged over all

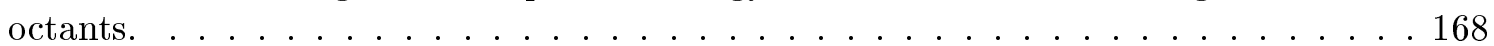

C.5 Table of correction factors. . . . . . . . . . . . . . . . . . . 169

C.6 Table of systematic uncertainties. . . . . . . . . . . . . . . . 170 


\section{List of Figures}

1.1 A phenomenological view of the $q \bar{q}$ sea within the proton. . . . . . . . . . . 3

1.2 Tree-level amplitudes for elastic electron-proton scattering. . . . . . . . . . . . . 6

1.3 One-quark electroweak radiative corrections. . . . . . . . . . . . . . . . 15

1.4 Multi-quark electroweak radiative corrections. . . . . . . . . . . . . . 15

1.5 Examples of hadronic models used to compute the strangeness content of the proton. 19

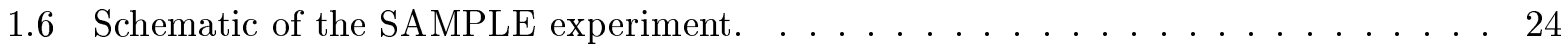

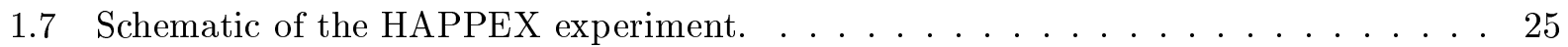

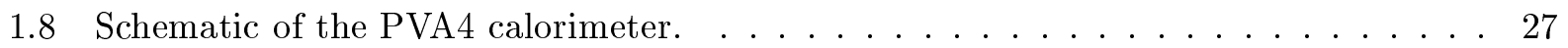

1.9 Summary of previous experiments conducted at $Q^{2}=0.1(\mathrm{GeV} / \mathrm{c})^{2}$. . . . . . . 28

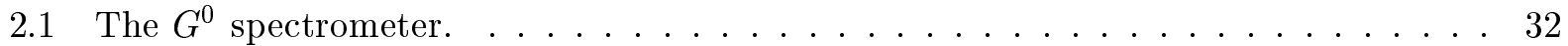

2.2 A schematic of the JLab accelerator from the source to the three halls. . . . . . . 33

2.3 A schematic of the injector laser table setup . . . . . . . . . . . . . . 34

2.4 Energy differences between the valence and conduction bands for the bulk (a) and strained (b) GaAs crystals. . . . . . . . . . . . . . . . . . 36

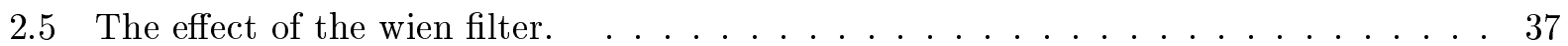

$2.6 G^{0}$ luminosity detector. The upstream detectors are in the even octants and are at a scattering angle of $2.0^{\circ}$. The downstream detectors are in the odd octants and are at a scattering angle of $1.2^{\circ} \ldots \ldots \ldots \ldots \ldots \ldots$

2.7 Plot showing the analyzing power of the cathode. . . . . . . . . . . . . 45

2.8 Adiabatic damping factor seen for different locations along the accelerator and in Hall C. . . . . . . . . . . . . . . . . . . . . . 46

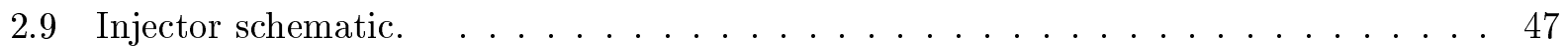

2.10 Phasespace ellipse. . . . . . . . . . . . . . . . . . . . 48

2.11 Laser intensity modulation through the use of an IA cell. . . . . . . . . . . . . 50

2.12 Schematic of the beam feedback system. . . . . . . . . . . . . . . 51

$2.13 \mathrm{HC}$ differences integrated over several hours. . . . . . . . . . . . . . . . 54

2.14 Final parity quality results for the $G^{0}$ forward angle measurement. . . . . . . . . . 55

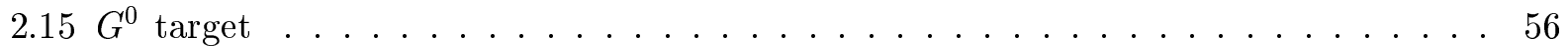

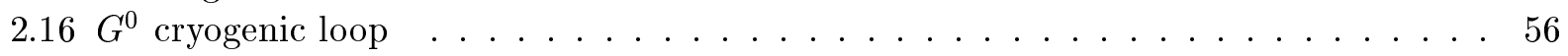

$2.17 G^{0}$ target manifold $\ldots \ldots \ldots \ldots \ldots \ldots \ldots \ldots$

2.18 FPD asymmetry width as a function of raster size. . . . . . . . . . . . 60

$2.19 G^{0}$ SMS cryogenic circuit. . . . . . . . . . . . . . . . 64

$2.20 G^{0}$ SMS electrical circuit. . . . . . . . . . . . . . . . . . . 65

2.21 Location of feedthrough ports. . . . . . . . . . . . . . . . 67

2.22 SMS control system. . . . . . . . . . . . . . . . . . . 68

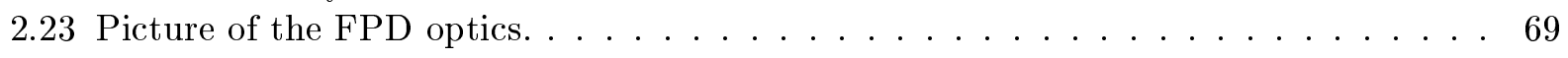


2.24 Data flowchart through the NA and FR electronics. . . . . . . . . . . . . 71

3.1 Sample tof spectra for octant 2 detector $8 \ldots \ldots \ldots \ldots$

3.2 Ratio of measured asymmetry width $\left(\sigma_{\text {meas }}\right)$ and the width calculated from counting

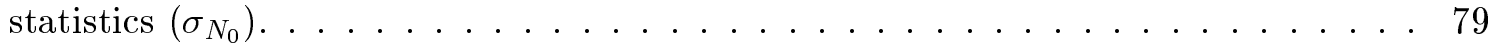

3.3 Tracking of elastic proton scattering through the G0GEANT simulation. . . . . . . 81

3.4 Comparison of detector rates between simulation and data for magnet current at 5015 A. . . . . . . . . . . . . . . . . . . 82

3.5 Detectors 15 and 16 elastic proton rate vs magnet current. . . . . . . . . . . 83

3.6 Comparison of pion-elastic proton tof separation as a function of detector. . . . . . 84

3.7 Leakage rates (blue) compared to the overall $G^{0}$ rates (black) . . . . . . . . . . . 88

3.8 Comparison of deduced and measured leakage current and asymmetry. . . . . . . . 90

3.9 Leakage current (top) and charge asymmetry (bottom) for the forward angle production run. . . . . . . . . . . . . . . . . . . . 91

3.10 Correlation plots for $A_{\text {meas }}$ and $A_{\text {false }}$ for the elastic proton cut and cut3. $\quad \ldots . .92$

3.11 Leakage position differences vs run-number. . . . . . . . . . . . . 93

3.12 Correlation between the measured asymmetry and the charge asymmetry. . . . . . 95

3.13 NA deadtime corrections. . . . . . . . . . . . . . . . . . 96

3.14 Fractional yield vs beam position. . . . . . . . . . . . . . . . . . . . 99

3.15 Linear regression slopes calculated from both NBM (Natural Beam Motion) and CP

(Coil Pulsing) runs for both IHWP IN and OUT. . . . . . . . . . . . . . 100

3.16 False asymmetries from multiple linear regression of $\mathrm{HC}$ beam fluctuations. . . . . . 101

3.17 Detectors 1 and 14 flyswatter and tungsten radiator data. . . . . . . . . . . . 103

3.18 Detectors 1 and 14 aluminum frame data. . . . . . . . . . . . . . . . 104

3.19 Rates vs. W seen from the MAID simulation. . . . . . . . . . . . . . 105

3.20 Detectors 1 and 14 empty target data and simulation. . . . . . . . . . . . . 106

3.21 LH2 asymmetries as a function of detector. . . . . . . . . . . . . . 107

3.22 Empty target and aluminum frame data. . . . . . . . . . . . . . . . . . . . . . . . . . . . . . . . . .

3.23 Comparison between simulation and SAPHIR data. . . . . . . . . . . . . . 109

3.24 Proton rate from hyperon decay simulated through G0GEANT. . . . . . . . . . . 110

3.25 Raw measured asymmetries and simulated hyperon asymmetries. . . . . . . . . . 111

3.26 Yield fit for octant 2, detector $8 \ldots \ldots \ldots \ldots \ldots$

3.27 Asymmetry fit for octant 2, detector 8. . . . . . . . . . . . . . 114

3.28 A lozenge shape is chosen as a conservative estimate of the upper and lower bounds

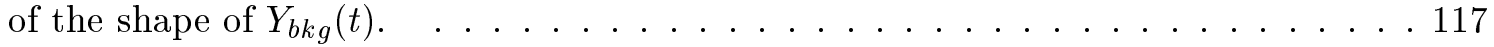

3.29 Asymmetry fit for octant 2 , detector $15 \ldots \ldots \ldots$. . . . . . . . . . 120

3.30 Background asymmetries for detectors 12 through $14 \mathrm{a}$, and detector 16 . . . . . . 121

3.31 Detector 15 elastic asymmetry vs octant. . . . . . . . . . . . . . . . . . . 122

3.32 Polarization vs run during the $G^{0}$ forward angle measurement. . . . . . . . . . . 129

3.33 Transverse asymmetry measurement. . . . . . . . . . . . . . . . 131

3.34 Amplitudes for virtual internal radiative corrections. . . . . . . . . . . . . . . . 132

3.35 Amplitudes for real internal radiative corrections. . . . . . . . . . . . . . . 133

3.36 Effect of radiative corrections to the measured asymmetry. . . . . . . . . . . . 134

3.37 Measured and corrected asymmetries. . . . . . . . . . . . . . . 135

$4.1 G_{E}^{s}+\eta G_{M}^{s}$ vs $Q^{2} \ldots \ldots \ldots \ldots \ldots \ldots \ldots \ldots \ldots$

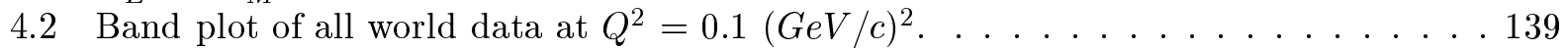

4.3 Band plot of all world data at $Q^{2}=0.23(\mathrm{GeV} / \mathrm{c})^{2} \ldots \ldots \ldots \ldots$ 
4.4 Band plot of all world data at $Q^{2}=0.477(\mathrm{GeV} / \mathrm{c})^{2} \ldots \ldots \ldots \ldots 14 \ldots$

A.1 The Testlab lasertable setup. . . . . . . . . . . . . . . . . . . . . 144

A.2 PZT slopes measured over time on the Testlab laser table. . . . . . . . . . . . . . 145

A.3 Beam orthogonality as seen in the injector. . . . . . . . . . . . . 146

A.4 Orthogonality of the PZT signals as seen in Hall C. . . . . . . . . . . . . . . . . 147

A.5 Variation of the width of the y position differences as two quadrupole magnets (3C17 and $3 \mathrm{C} 19)$ are adjusted. . . . . . . . . . . . . . . 148

A.6 Correlation between $\mathrm{x}$ and $\mathrm{y}$ position differences and charge asymmetry for an IA and an RHWP scan. . . . . . . . . . . . . . . . . . . . . . . 149

A.7 Feedback performance before the correction of the feedback matrix. . . . . . . . 150

A.8 Feedback performance after the correction of the feedback matrix. . . . . . . . . 151

B.1 $Y_{0}$ vs Beam current. No visible correlation is seen. . . . . . . . . . . . . 153

B.2 $Y_{0}$ vs Beam position. No visible correlation is seen. . . . . . . . . . . 153

B.3 White noise spectra for one of the NA detectors. . . . . . . . . . . . . 154

B.4 Improvement of DNL from the first to the second engineering run. . . . . . . . . 155

B.5 Example of a spike in the FR electronics. . . . . . . . . . . . . 156

B.6 FR and NA detector efficiency. . . . . . . . . . . . . . . . . 157

C.1 Octant 2 Detector 1. Asymmetries (black), and yields (blue) . . . . . . . . 158

C.2 Octant 2 Detector 2. Asymmetries (black), and yields (blue). . . . . . . . . . 159

C.3 Octant 2 Detector 3. Asymmetries (black), and yields (blue). . . . . . . . . . . 159

C.4 Octant 2 Detector 4. Asymmetries (black), and yields (blue) . . . . . . . . . . 160

C.5 Octant 2 Detector 5. Asymmetries (black), and yields (blue). . . . . . . . . . 160

C.6 Octant 2 Detector 6. Asymmetries (black), and yields (blue) . . . . . . . . . . . 161

C.7 Octant 2 Detector 7. Asymmetries (black), and yields (blue) . . . . . . . . . . . 161

C.8 Octant 2 Detector 8. Asymmetries (black), and yields (blue) . . . . . . . . . . 162

C.9 Octant 2 Detector 9. Asymmetries (black), and yields (blue) . . . . . . . . . . 162

C.10 Octant 2 Detector 10. Asymmetries (black), and yields (blue). . . . . . . . . 163

C.11 Octant 2 Detector 11. Asymmetries (black), and yields (blue). . . . . . . . . 163

C.12 Octant 2 Detector 12. Asymmetries (black), and yields (blue). . . . . . . . . . . 164

C.13 Octant 2 Detector 13. Asymmetries (black), and yields (blue). . . . . . . . . . . 164

C.14 Octant 2 Detector 14. Asymmetries (black), and yields (blue). . . . . . . . . . 165

C.15 Octant 2 Detector 15. Asymmetries (black), and yields (blue). . . . . . . . . 165

C.16 Octant 2 Detector 16. Asymmetries (black), and yields (blue). . . . . . . . . 166 


\section{Chapter 1}

\section{Introduction}

Since the discovery of the nucleus by Rutherford, significant strides have been made in advancing one of the primary goals of physics; to understand the nature of matter at smaller and smaller distance scales. This breakthrough discovery in 1911 has eventually fostered the understanding that what surrounds us in everyday life is a composition of structures at many scales. From the probing of the internal structure of atoms and nucleons to the eventual discovery of quarks, a standard model (SM) of physics has emerged which, albeit incomplete, attempts to unify the vast variety of fundamental and composite particles that have been observed and the laws that govern their interactions.

Since the advent of the electroweak theory in the 1970's, which describes the electromagnetic and weak interactions of fundamental and composite particles alike, significant efforts have been made to understand the strong interaction which binds hadronic matter together. Quantum chromodynamics (QCD) attempts to do this by postulating that quarks interact with each other through the exchange of color gauge fields, or gluons. Furthermore, a quark-gluon sea exists within hadronic matter, where quarks and antiquarks can fluctuate in and out of existence by pair-producing from the gluon fields. The theory is capable of describing the interactions of quarks at short distance scales (high energy) using a converging perturbation expansion. However, at large distance scales (low energy) of order the size of a nucleon, the strong coupling constant $\alpha_{s}$ is too large for the perturbation expansion to converge. This non-perturbative nature of the interaction of quarks and gluons has not yet been fully understood, and stands in the path of revealing some of the most fundamental properties of the internal structure of the nucleon. The work presented here will give insight into the structure of the nucleon based on measurements of the role of strange quarks to the 
charge and magnetization distribution of the proton. This measurement gives direct insight into the properties of the quark-gluon sea which to this date is not well understood.

The rest of this chapter will discuss the formalism of parity-violating electron scattering and its connection to the strange quark contribution to proton structure, various theoretical calculations of strange form factors, and descriptions of previous parity-violating experiments.

\subsection{The Strange Sea}

The first indication that the proton may have some internal structure came when Frisch and Stern made a measurement of the proton's magnetic moment [1]. It was believed until then that as a point-like Dirac particle, the proton's magnetic moment is $\mu_{p}=\frac{e}{2 m_{p}}$, defined as one nuclear magneton (n.m.). The fact that their results showed a proton magnetic moment to be between 2 and 3 n.m. challenged the preconception that the proton is a point-like particle.

The present day description of the proton is that of a composite particle consisting of three valence quarks (two up quarks and a down quark) interacting through the exchange of gluons. The quarks are thought to be point-like particles, each having a magnetic moment $\mu_{u / d}=Q_{u, d} \frac{e}{2 m_{u, d}}$. Calculations of the proton magnetic moment have been made within the framework of the quark model. However, the precision attained through the various theoretical calculations lags behind in comparison to the value of $\mu_{p}$ determined through experiment. The problem resides, again, in the difficulty of calculating low-energy properties of hadrons in QCD, due to its large coupling constant at that energy scale.

The valence quarks are surrounded by a quark-gluon sea where quark-antiquark pairs can be created and annihilated through pair production in the gluon field. Any strange quark that may contribute to the structure of the proton must originate from this sea. A schematic of this process can be seen in Fig. 1.1.

Apart from experiments based on parity-violation, which is the topic of this dissertation, there have been numerous other experiments that probe the contribution of strange quarks to proton structure. The analysis of the pion-nucleon $\sigma$ term [2] is one of the original motivations for probing the strange sea within the nucleon. In this analysis, the nucleon mass is separated into each of its 


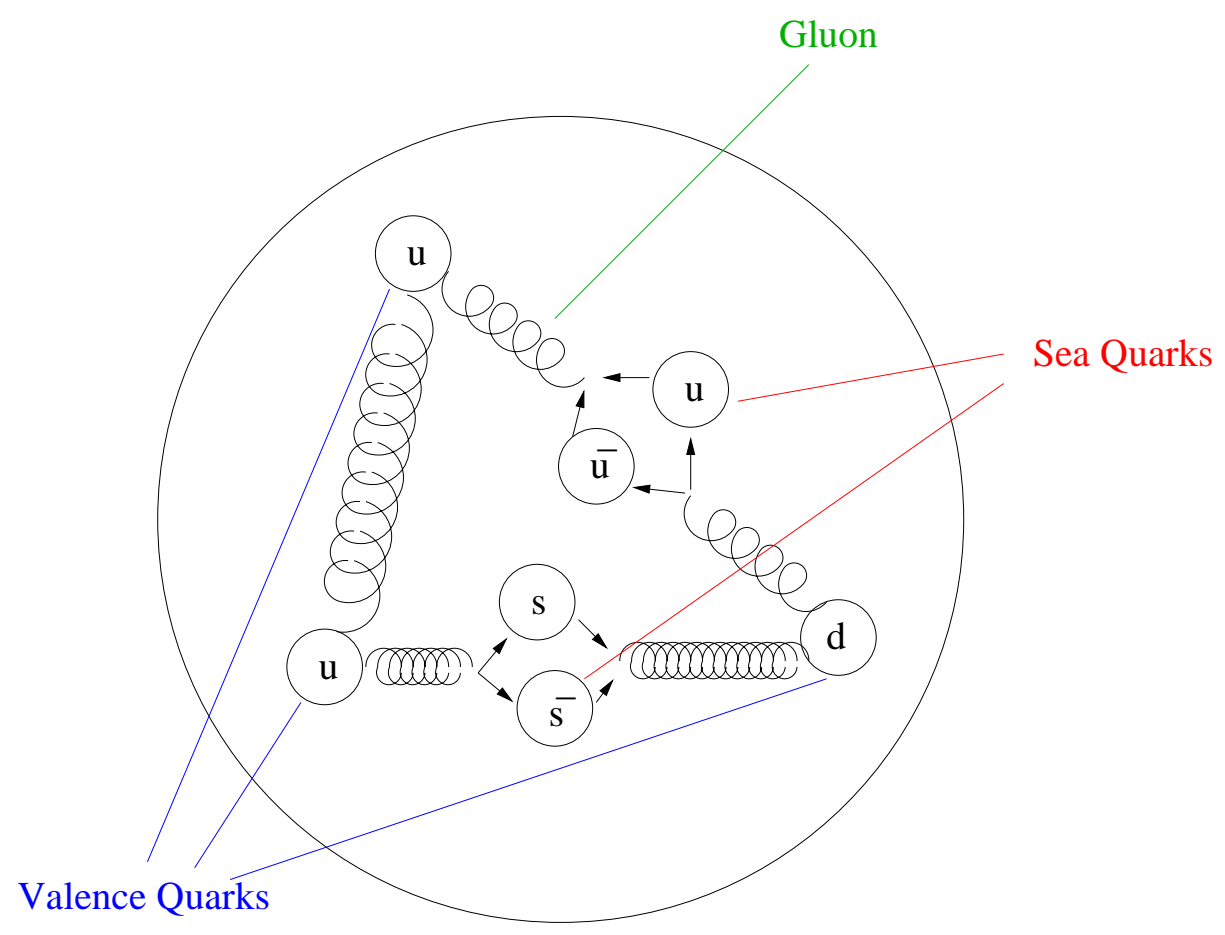

Figure 1.1: A schematic view of the $q \bar{q}$ sea within the proton. The pair produced(and subsequently annihilated) $q \bar{q}$ pairs may contribute significantly to the electromagnetic properties of the proton.

valence and non-valence quark components

$$
M_{N}=M_{0}+\sigma_{s}+\sigma
$$

where $M_{N}$ is the nucleon mass, $M_{0}$ is the mass of the gluon sea and the $q \bar{q}$ condensate, and

$$
\begin{gathered}
\sigma_{s}=\frac{1}{2 M_{N}}\left\langle N\left|m_{s} s \bar{s}\right| N\right\rangle \\
\sigma=\frac{1}{2 M_{N}}\left\langle N\left|\frac{m_{u}+m_{d}}{2}(u \bar{u}+d \bar{d})\right| N\right\rangle
\end{gathered}
$$

where $m_{u}, m_{d}$, and $m_{s}$ are the $\mathrm{u}, \mathrm{d}$, and s quark masses. Two constraints can be imposed on the above relations. One is that $\sigma$ can be determined from $\pi \mathrm{N}$ scattering. The other is that the hyperon masses give insight into the contributions from the quark masses. In the latter constraint, if one were to assume $\langle N|s \bar{s}| N\rangle=0$, then the two constraints would give equivalent results. The fact that they do not agree indicates the existence of the $s \bar{s}$ contribution to nucleon structure, with an 
overall strange quark contribution of

$$
\frac{\langle N|s \bar{s}| N\rangle}{\langle N|(u \bar{u}+d \bar{d})| N\rangle} \simeq 0.2
$$

However, it should be added that the results from the pion-nucleon $\sigma$ term has evolved over time, and represents a work still in progress.

During the 1980's, the European Muon Collaboration (EMC) [3] measured the spin contribution of constituent quarks to the overall spin of the proton. The measurement, combined with measurements performed at SLAC [4] found that the three lightest quarks contribute to proton spin by determining the spin-dependent structure function, $g_{1}^{p}$, of the proton,

$$
\int_{0}^{1} g_{1}^{p} d x=0.126 \pm 0.010 \pm 0.015
$$

which is in contradiction with the value of $0.189 \pm 0.005$ calculated from the Ellis-Jaffe sum rule. However, the calculation through the Ellis-Jaffe sum rule neglects contributions from the strange quark.

From the EMC/SLAC measurements, the strange quark contribution to the proton spin can be extracted, assuming $S U(3)_{f}$ symmetry. By relating the structure function in Eqn. 1.5 to the antisymmetric (F) and symmetric (D) SU(3) couplings, the individual quark contributions to proton spin are found

$$
\begin{aligned}
& \left\langle S_{z}\right\rangle_{u} \frac{1}{2}(\Delta u+\Delta \bar{u})=0.391 \pm 0.016 \pm 0.023 \\
& \left\langle S_{z}\right\rangle_{d} \frac{1}{2}(\Delta d+\Delta \bar{d})=-0.236 \pm 0.016 \pm 0.023 \\
& \left\langle S_{z}\right\rangle_{s} \frac{1}{2}(\Delta s+\Delta \bar{s})=-0.095 \pm 0.016 \pm 0.023
\end{aligned}
$$

The result shows that the strange quarks are polarized anti-parallel to the proton spin, and contribute about $20 \%$ in magnitude of the total proton spin of $\frac{1}{2}$.

Another probe of strange quarks within the nucleon is that from deep-inelastic neutrino scattering. The NuTeV [5] experiment detects final states with oppositely charged muons (dimuons) to study charm production as well as the strange sea. The $s(x)$ and $\bar{s}(x)$ momentum distributions 
were parametrized in such a way as to assure that it does not violate the flavor sum rule. This is achieved by inserting a point of zero-crossing, $x_{0}$, into the parametrization to enforce the condition that $\int(s(x)-\bar{s}(x)) d x=0$. The resulting $s(x)$ and $\bar{s}(x)$ momentum distributions are

$$
\begin{aligned}
s^{+}\left(x, Q_{0}\right) & =\kappa^{+}(1-x)^{\alpha^{+}} x^{\gamma^{+}}\left[\bar{u}\left(x, Q_{0}\right)+\bar{d}\left(x, Q_{0}\right)\right] \\
s^{-}\left(x, Q_{0}\right) & =s^{+}(x) \tanh \left[\kappa^{-}(1-x)^{\alpha^{-}} x^{\gamma^{-}}\left(1-\frac{x}{x_{0}}\right)\right] \\
s & =\frac{s^{+}+s^{-}}{2} \quad \bar{s}=\frac{s^{+}-s^{-}}{2}
\end{aligned}
$$

where $Q_{0}$ is the momentum transfer, $x=\frac{-q^{2}}{2 p \cdot q}$ is the fraction of the nucleon momentum carried by the quark in the infinite momentum frame, and $\kappa^{+,-}, \alpha^{+,-}$, and $\gamma^{+,-}$are constants associated with the parameterization. From the above parameterizations, the asymmetry in the $s(x)$ and $\bar{s}(x)$ momentum distributions is found to be

$$
\int x[s(x)-\bar{s}(x)] d x=-0.0009 \pm 0.0014
$$

which is consistent with zero.

In addition to $\mathrm{NuTeV}$, which parameterizes the strange quark's momentum distribution, and polarized DIS experiments which probe the strange quark's spin contribution, there is a class of experiments which attempt to determine the strange quark's contribution to the charge $\left(G_{E}^{s}\right)$ and magnetization $\left(G_{M}^{s}\right)$ distributions within the proton. The form factors, $G_{E}^{s}$ and $G_{M}^{s}$, will be discussed further in the next section. The $G^{0}$ experiment and numerous other parity-violating (PV) electron-proton scattering experiments directly quantify these distributions by determining the elastic response of the proton to a neutral weak probe [6] [7] [8]. Neglecting heavy quarks, the u, $\mathrm{d}$, and s quark contributions to nucleon structure are separated by measuring the weak properties of the nucleon, and comparing it with the well-known electromagnetic properties. PV experiments measure the asymmetry in elastic electron-proton scattering for opposing incident electron helicity states

$$
A=\frac{\sigma_{+}-\sigma_{-}}{\sigma_{+}+\sigma_{-}}
$$

where $\sigma_{+(-)}$denote the cross sections for the positive(negative) electron helicities, and $A$ is the 
asymmetry. The asymmetry measured in PV experiments at forward scattering angles can measure a linear combination of the charge and magnetization components of the strange quark contribution. This dissertation will focus on the forward angle measurement of the $G^{0}$ experiment which determines the linear combination over a range of length scales within the nucleon.

\subsection{Formalism}

This section will focus on the theoretical framework upon which PV experiments are based. The various e-p scattering amplitudes that contribute to the measurement will be described, and the hadronic and quark form factors which characterize the charge and magnetic structure of the nucleon will be introduced.

At tree level, PV electron-proton scattering can be described by a photon $(\gamma)$ and a neutral weak boson $(\mathrm{Z})$ exchange as seen in Fig. 1.2. The interaction of the electron and the proton is given
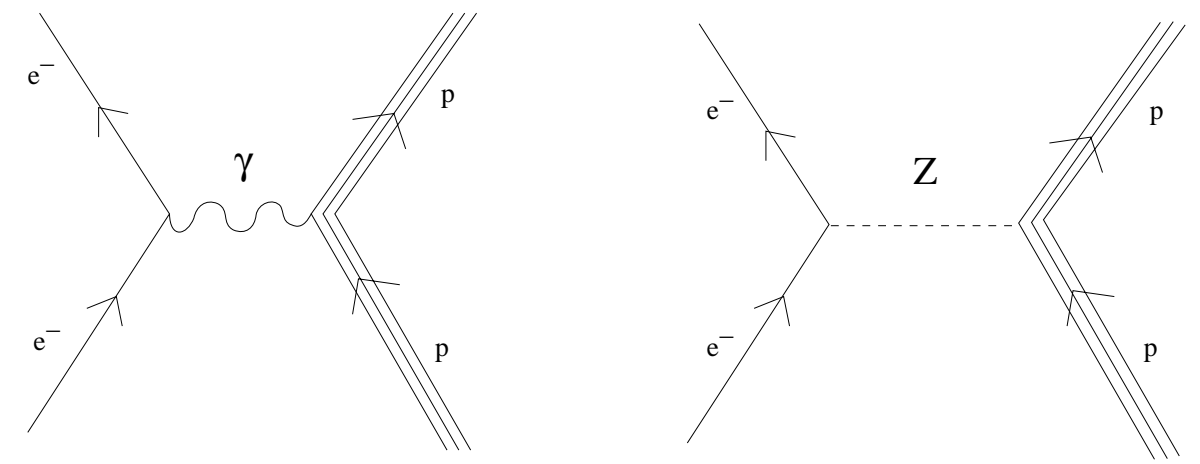

Figure 1.2: Tree-level amplitudes for elastic electron-proton scattering. The photon and the neutral weak boson exchange are the basic modes of interaction.

by their respective vector and axial vector $(\mathrm{V}-\mathrm{A})$ coupling to these neutral currents. The invariant amplitudes for these tree level diagrams can be written by combining the coupling of the neutral currents to a point-like Dirac fermion $\left(j_{\gamma}^{\mu}\right.$ and $\left.j_{Z}^{\mu 5}\right)$ such as an electron, and to a composite particle $\left(J_{\gamma}^{\mu}\right.$ and $\left.J_{Z}^{\mu 5}\right)$ like a proton [10] [12]. Here, the superscript $\mu$ is associated with the vector current, and $\mu 5$ denotes an axial-vector current. The electromagnetic amplitude can be written in terms of the leptonic and hadronic vector currents

$$
M_{\gamma}=\left\langle\psi_{e, f}\left|e j_{\gamma}^{\mu}\right| \psi_{e, i}\right\rangle \frac{g_{\mu \nu}}{Q^{2}}\left\langle\psi_{p, f}\left|e J_{\gamma}^{\nu}\right| \psi_{p, i}\right\rangle=\frac{4 \pi \alpha g_{\mu \nu}}{Q^{2}} j_{\gamma}^{\mu} J_{\gamma}^{\nu}
$$


where $e^{2}=4 \pi \alpha, g_{\mu \nu}$ is the metric tensor, $j_{\gamma}^{\mu}=\left\langle\psi_{e, f}\left|j_{\gamma}^{\mu}\right| \psi_{e, i}\right\rangle$ is the lepton electromagnetic current, and $J_{\gamma}^{\mu}=\left\langle\psi_{p, f}\left|J_{\gamma}^{\mu}\right| \psi_{p, i}\right\rangle$ is that for a proton. The fine structure constant, $\alpha$, characterizes the strength of the electromagnetic amplitude. For a point-like Dirac fermion such as a lepton, the transition can be written as a vector current

$$
j_{\gamma}^{\mu}=\bar{u} \gamma^{\mu} u
$$

$\bar{u}$ and $u$ are the lepton spinors. In the case of a proton, which is not a point-like particle, the current is written in terms of its form factors

$$
J_{\gamma}^{\mu}=\bar{U}\left(F_{1}^{\gamma}\left(q^{2}\right) \gamma^{\mu}+\frac{\kappa}{2 M} F_{2}^{\gamma}\left(q^{2}\right) i \sigma^{\mu \nu} q_{\nu}\right) U
$$

where $F_{1}^{\gamma}\left(q^{2}\right)$ and $F_{2}^{\gamma}\left(q^{2}\right)$ are the Dirac and Pauli electromagnetic form factors, respectively, and $\bar{U}$ and $U$ are the nucleon spinors. These form factors can be written in terms of the individual quark flavors, as will be shown later. They characterize the structure of the hadron in question, and are dependent on the invariant four momentum transfer, $Q^{2}$. The $F_{1}$ form factor converges to one, and the $F_{2}$ converges to $\frac{1}{\tau}$ as $Q^{2} \rightarrow 0$, since the compositeness of the proton cannot be seen at such large distance scales.

In the case of the neutral weak interaction, the invariant amplitude in Eqn. 1.14 can be re-written with the electromagnetic currents replaced by the neutral weak V-A currents. Furthermore, the electromagnetic coupling constant, $\alpha$, is replaced by the weak coupling constant, $G_{F}$, and the electromagnetic currents, $j_{\gamma}^{\mu}$ and $J_{\gamma}^{\nu}$ are replaced by the neutral weak currents, $j_{Z}^{\mu 5}$ and $J_{Z}^{\nu 5}$

$$
M_{Z}=-\frac{G_{F}}{2 \sqrt{2}} g_{\mu \nu} j_{Z}^{\mu 5} J_{Z}^{\nu 5}
$$

where $g_{\mu \nu}$ is the metric tensor. As in the case with the photon exchange, the neutral weak current of leptons and hadrons, $j_{Z}^{\mu 5}$ and $J_{Z}^{\nu 5}$, can be expressed with the relevant $\gamma$-matrices

$$
j_{Z}^{\mu 5}=\bar{u} \frac{1}{2}\left(g_{V} \gamma^{\mu}-g_{A} \gamma^{\mu} \gamma_{5}\right) u
$$

where $g_{V}$ and $g_{A}$ are the vector and axial vector weak charges. The electromagnetic and weak 
charge [10] for a few of the fundamental fermions can be seen in Table 1.1. For the hadron

\begin{tabular}{|c|c|c|c|}
\hline Particle & $e^{\gamma}$ & $g_{V}^{Z}$ & $g_{A}^{Z}$ \\
\hline$e^{-}$ & -1 & $-1+4 \sin ^{2} \theta_{w}$ & 1 \\
\hline $\mathrm{u}, \mathrm{c}, \mathrm{t}$ & $\frac{2}{3}$ & $1-\frac{8}{3} \sin ^{2} \theta_{w}$ & -1 \\
\hline $\mathrm{d}, \mathrm{s}, \mathrm{b}$ & $-\frac{1}{3}$ & $-1+\frac{4}{3} \sin ^{2} \theta_{w}$ & 1 \\
\hline
\end{tabular}

Table 1.1: Electromagnetic and weak charges.

$$
J_{Z}^{\mu}=\bar{U}\left(F_{1}^{Z}\left(q^{2}\right) \gamma^{\mu}+\frac{\kappa}{2 M} F_{2}^{Z}\left(q^{2}\right) i \sigma^{\mu \nu} q_{\nu}+G_{A}^{e} \gamma^{\mu} \gamma_{5}\right) U
$$

where in addition to the weak counterparts, $F_{1}^{Z}$ and $F_{2}^{Z}$, to the Dirac and Pauli form factors, the neutral weak axial form factor, $G_{A}^{e}$, is introduced, and is associated with the axial vector component of the current. The overall tree-level amplitude for electron-proton scattering is simply the sum of the electromagnetic $\left(M_{\gamma}\right)$ and weak amplitudes $\left(M_{Z}\right)$

$$
M=M_{\gamma}+M_{Z}
$$

as shown in Fig. 1.2. PV electron-proton scattering is sensitive to the neutral current amplitude, as will be shown later in the discussion of the $\mathrm{PV}$ asymmetry. The form factors $F_{1}$ and $F_{2}$ are often re-written to separate the electric and magnetic components (in both the electromagnetic and neutral weak cases) [10]

$$
\begin{aligned}
G_{E, p}^{\gamma, Z} & =F_{1, p}^{\gamma, Z}-\tau F_{2, p}^{\gamma, Z} \\
G_{M, p}^{\gamma, Z} & =F_{1, p}^{\gamma, Z}+F_{2, p}^{\gamma, Z}
\end{aligned}
$$

where $\tau=\frac{Q^{2}}{4 M_{p}}, F_{1, p}^{\gamma, Z}$ and $F_{2, p}^{\gamma, Z}$ are the electromagnetic and weak Dirac and Pauli form factors for the proton, and $G_{E, p}^{\gamma, Z}$ and $G_{M, p}^{\gamma, Z}$ are called the Sachs form factors.

The Sachs form factors describe the electric and magnetic structure of the proton, and are of primary importance in parity-violating experiments. It is often useful to describe these form factors in the Breit frame, where $p_{i}=-p_{f}$, and no energy is transferred to the proton. In this frame, the Sachs form factors are, roughly speaking ${ }^{1}$, the Fourier transforms of the charge and magnetization

\footnotetext{
${ }^{1}$ There is some ambiguity due to the presumably highly relativistic motion of the quarks in the proton - the
} 
distributions.

In the limit $Q^{2} \rightarrow 0$, the Sachs form factors represent the static electric, magnetic, and axial properties of the proton

$$
\begin{aligned}
& G_{E, p}^{\gamma, Z}\left(Q^{2}=0\right)=q_{p}^{\gamma, Z} \\
& G_{M, p}^{\gamma, Z}\left(Q^{2}=0\right)=\mu_{p}^{\gamma, Z} \\
& G_{A}^{e}\left(Q^{2}=0\right)=q_{A}^{Z}
\end{aligned}
$$

where $q_{p}^{\gamma, Z}, \mu_{p}^{\gamma, Z}$, and $q_{A}^{Z}$ are the electric and weak charge, the magnetic moment, and the axial charge of the proton, respectively. Furthermore, when the electromagnetic Sachs form factors, $G_{E, p}^{\gamma}$ and $G_{M, p}^{\gamma}$, are expanded in series assuming low $Q^{2}$, the mean square proton charge and magnetization radius, $\left\langle r_{E, M}^{2}\right\rangle$, can be calculated from the linear term in the expansion

$$
\begin{aligned}
& \left\langle r_{E}^{2}\right\rangle=-\left.6 \frac{d G_{E}^{\gamma}}{d Q^{2}}\right|_{Q^{2}=0} \\
& \left\langle r_{M}^{2}\right\rangle=-\left.6 \frac{d G_{M}^{\gamma}}{\mu_{p} d Q^{2}}\right|_{Q^{2}=0}
\end{aligned}
$$

For the proton, one of the more recent measurements of the charge radius is $0.895 \pm 0.018 \mathrm{fm}[9]$.

The objective of the various parity-violating experiments is to separate these Sachs form factors into the respective contributions from each of the quark flavors, $G_{E, M}^{u, \gamma}, G_{E, M}^{d, \gamma}, G_{E, M}^{s, \gamma}$. The flavor decomposition of the form factors can be deduced from the decomposition of the individual quark currents. Since quarks are point-like Dirac fermions, their coupling to both the electromagnetic and weak currents do not involve any additional form factors

$$
\begin{aligned}
& J_{p, \gamma}^{\mu}=\Sigma_{f} e_{f}^{\gamma} \overline{u_{f}} \gamma^{\mu} u_{f} \\
& J_{p, Z}^{\mu}=\Sigma_{f} g_{f}^{Z, V} \overline{u_{f}} \gamma^{\mu} u_{f}+g_{f}^{Z, A} \overline{u_{f}} \gamma^{\mu} \gamma_{5} u_{f}
\end{aligned}
$$

where the subscript $f$ indicates the three lightest quark flavors, $u_{f}$ and $\overline{u_{f}}$ are quark spinors, and $e_{f}^{\gamma}, g_{f}^{Z, V}$, and $g_{f}^{Z, A}$ are the electromagnetic charge and weak vector and axial charges. The sum over the quark flavors implicitly includes both quarks and their anti-quarks. The electromagnetic non-relativistic Fourier transform is not really appropriate. 
form factors can then be divided up into a sum of the quark contributions

$$
\begin{aligned}
& G_{E, M}^{\gamma, p}=\Sigma_{f} e_{f}^{\gamma} G_{E, M}^{f} \\
& G_{E, M}^{Z, p}=\Sigma_{f} g_{f}^{Z, V} G_{E, M}^{f} \\
& G_{A}^{e, p}=\Sigma_{f} g_{f}^{Z, A} G_{A}^{f}
\end{aligned}
$$

where $G_{E, M}^{f}$ is the electromagnetic quark "form factor", and $G_{A}^{f}$ is the axial quark form factor. The quark form factors shown here do not imply the existence of any internal structure to the quarks, as the electromagnetic proton form factors do for the protons. This can be seen from the fact that the quark currents do not include any form factors which could account for any compositeness. Rather, the quark form factors merely show the contributions that each quark makes to the overall structure of the proton.

The asymmetry measured in parity-violation is sensitive to the product of $G_{E, M}^{\gamma, p}$ and $G_{E, M}^{Z, p}$. Since $G_{E, M}^{\gamma, p}$ is well known, parity-violation therefore measures $G_{E, M}^{Z, p}$. The electromagnetic form factor for the proton, $G_{E, M}^{\gamma, p}$, is well known from previous experiments. From charge symmetry, the neutron electromagnetic form factor, $G_{E, M}^{\gamma, n}$, can be determined by interchanging the $\mathrm{u}$ and d flavors in $G_{E, M}^{\gamma, p}$. This is under the assumption that the $\mathrm{u}$ quark distribution in the proton is identical to the $\mathrm{d}$ quark distribution in the neutron, and vice-versa. Charge symmetry breaking occurs due to the differing masses of the $\mathrm{u}$ and $\mathrm{d}$ quarks and to electromagnetic effects (since the charges are different), but this effect is seen to be less than a $1 \%$ effect [11] and is deemed tolerable since the uncertainty for the $G^{0}$ experiment is between 5 and $10 \%$.

With the proton and neutron electromagnetic form factors $\left(G_{E, M}^{\gamma, p}, G_{E, M}^{\gamma, n}\right)$ known, and the neutral weak proton form factor $\left(G_{E, M}^{Z, p}\right)$ to be measured through parity-violation, three values will be known that are necessary to separate the three quark flavor form factors. These proton form factors can 
be explicitly written in terms of the quark form factors

$$
\begin{aligned}
G_{E, M}^{\gamma, p} & =\frac{2}{3} G_{E, M}^{u, p}-\frac{1}{3} G_{E, M}^{d, p}-\frac{1}{3} G_{E, M}^{s, p} \\
G_{E, M}^{\gamma, n} & =\frac{2}{3} G_{E, M}^{d, p}-\frac{1}{3} G_{E, M}^{u, p}-\frac{1}{3} G_{E, M}^{s, p} \\
G_{E, M}^{Z, p} & =\left(1-\frac{8}{3} \sin ^{2} \theta_{w}\right) G_{E, M}^{u, p}-\left(1-\frac{4}{3} \sin ^{2} \theta_{w}\right)\left(G_{E, M}^{d, p}+G_{E, M}^{s, p}\right) \\
G_{A}^{e}= & -\left(G_{A}^{u}-G_{A}^{d}\right)+G_{A}^{s}
\end{aligned}
$$

where $\theta_{w}$ is the Weinberg mixing angle, $G_{E, M}^{u, p}, G_{E, M}^{d, p}$, and $G_{E, M}^{s, p}$ are the u,d, and s quark contributions to the proton's electromagnetic form factors, and $G_{A}^{u}, G_{A}^{d}, G_{A}^{s}$ represent their contributions to the axial form factor. Inverting the above relations give the quark vector form factors

$$
\begin{aligned}
& G_{E, M}^{u}=\left(3-4 \sin ^{2} \theta_{w}\right) G_{E, M}^{\gamma, p}-G_{E, M}^{Z, p} \\
& G_{E, M}^{d}=\left(2-4 \sin ^{2} \theta_{w}\right) G_{E, M}^{\gamma, p}+G_{E, M}^{\gamma, n}-G_{E, M}^{Z, p} \\
& G_{E, M}^{s}=\left(1-4 \sin ^{2} \theta_{w}\right) G_{E, M}^{\gamma, p}-G_{E, M}^{\gamma, n}-G_{E, M}^{Z, p}
\end{aligned}
$$

Determining the strange electromagnetic form factors, $G_{E, M}^{s}$, is the ultimate goal of this dissertation.

The strange form factor can be analyzed in a manner similar to the electromagnetic proton form factor by observing how it behaves in the limit $Q^{2} \rightarrow 0$

$$
\begin{aligned}
& \left\langle r_{s}^{2}\right\rangle=-\left.6 \frac{d G_{E, M}^{s}}{d Q^{2}}\right|_{Q^{2}=0} \\
& \mu_{s}=G_{M}^{s}\left(Q^{2}=0\right)
\end{aligned}
$$

where $\left\langle r_{s}^{2}\right\rangle$ and $\mu_{s}$ are the strangeness radius and magnetic moment, respectively. In the limit where $Q^{2} \rightarrow 0, G_{E}^{s}=0$ since the total charge of the strange quarks/antiquarks in the proton is zero. These represent the strange quark contribution to the proton's charge radius and magnetic moments seen in Eqn. 1.27. They represent the static properties $\left(Q^{2}=0\right)$ of the proton, and theoretical calculations quantifying the strangeness properties of the proton often attempt to predict $\left\langle r_{s}^{2}\right\rangle$ and $\mu_{s}$. 


\section{$G^{0}$ : The Flavor Singlet Form Factor}

The flavor decomposition of the neutral weak proton form factor can also be re-written in terms of the $\mathrm{SU}(3)$ flavor basis. This allows a clear distinction of what new information is contained in the

measurement of $G_{E, M}^{Z, p}$. In the $\mathrm{SU}(3)$ flavor basis, the proton electromagnetic form factor can be written as a sum of its isovector and octet terms

$$
G_{E, M}^{\gamma, p}=G_{E, M}^{3, p}+\frac{1}{3} G_{E, M}^{8, p}
$$

where $G_{E, M}^{3, p}$ and $G_{E, M}^{8, p}$ are the isovector and octet form factors, respectively. These form factors in turn can be written in terms the quark contributions,

$$
\begin{aligned}
G_{E, M}^{3, p} & =\frac{1}{2}\left(G_{E, M}^{u, p}-G_{E, M}^{d, p}\right) \\
G_{E, M}^{8, p} & =\frac{1}{2 \sqrt{3}}\left(G_{E, M}^{u, p}+G_{E, M}^{d, p}-2 G_{E, M}^{s, p}\right)
\end{aligned}
$$

With the above relations, $G_{E, M}^{Z, p}$ as seen in Eqn. 1.33 can be re-written in terms of the electromagnetic form factor, $G_{E, M}^{\gamma, p}$, and a term containing the new information

$$
\begin{aligned}
G_{E, M}^{Z, p} & =\left(\frac{1}{2}-\sin ^{2} \theta_{w}\right) G_{E, M}^{3, p}+\left(\frac{1}{2 \sqrt{(3)}}-\frac{1}{3} \sin ^{2} \theta_{w}\right) G_{E, M}^{8, p}-\frac{1}{4} G_{E, M}^{0, p} \\
& =\left(\frac{1}{2}-\sin ^{2} \theta_{w}\right) G_{E, M}^{\gamma, p}-\frac{1}{4} G_{E, M}^{0, p}
\end{aligned}
$$

where $G_{E, M}^{0, p}$ is the flavor singlet proton form factor, which contains the strange quark form factor. In terms of the quark contributions, $G_{E, M}^{0, p}$ takes the following form

$$
G_{E, M}^{0, p}=\frac{1}{3}\left(G_{E, M}^{u, p}+G_{E, M}^{d, p}+G_{E, M}^{s, p}\right)
$$

Therefore, the $G^{0}$ experiment is sensitive to this flavor singlet proton form factor which is the additional information probed through parity-violation. 


\subsubsection{Parity-Violating Asymmetry}

The electromagnetic properties of the proton, as described by the photon-exchange amplitude in Eqn. 1.20 are well known, while the asymmetry in Eqn. 1.13 gives insight into the neutral weak amplitude. Vector currents such as photons conserve parity, while the neutral weak current, carrying equal parts vector and axial vector components, violate parity (space inversion) maximally. This property of the neutral weak interaction is of main interest to PV experiments. The factors in Eqn. 1.17, 1.18, and 1.19 that violate parity are the terms involving vector coupling of the $\mathrm{Z}$ to the electron and axial coupling to the proton, and vice-versa. However, the degree of parity violation seen in electron-proton scattering is diluted significantly by the electromagnetic amplitude. The cross section in e-p scattering is given by $d \sigma \propto\left|M_{\gamma}+M_{Z}\right|^{2}$. Thus, the asymmetry measured through parity violation is reduces to [13]

$$
A_{p}=\frac{\sigma_{+}-\sigma_{-}}{\sigma_{+}+\sigma_{-}}=\frac{M_{\gamma} M_{Z}}{\left|M_{\gamma}\right|^{2}}
$$

where $M_{\gamma}$ is larger than $M_{Z}$ by about a factor of $10^{5}$. Therefore, the asymmetries measured in these parity-violation experiments are of order parts per million.

The asymmetry measured in Eqn. 1.42 can be written in terms of the electromagnetic and neutral weak proton form factors [13]

$$
A_{p}=-\frac{G_{F} Q^{2}}{4 \pi \alpha \sqrt{2}} \frac{\epsilon G_{E}^{\gamma, p} G_{E}^{Z, p}+\tau G_{M}^{\gamma, p} G_{M}^{Z, p}-\left(1-4 \sin ^{2} \theta_{w}\right) \epsilon^{\prime} G_{M}^{\gamma, p} G_{A}^{e}}{\epsilon\left(G_{E}^{\gamma, p}\right)^{2}+\tau\left(G_{M}^{\gamma, p}\right)^{2}}
$$

where $\epsilon=\left(1+2(1+\tau) \tan ^{2} \frac{\theta_{e}}{2}\right)^{-1}, \epsilon^{\prime}=\sqrt{\tau(1+\tau)\left(1-\epsilon^{2}\right)}$, and $\tau=\frac{Q^{2}}{4 M_{p}}$. The dependence of the asymmetry on the angular kinematics is such that the measurement is sensitive to $G_{E}^{Z, p}$ and $G_{M}^{Z, p}$ in the forward angles, and sensitive to $G_{M}^{Z, p}$ and $G_{A}^{e}$ at backward scattering angles. This feature of the asymmetry is used to kinematically separate the electric and magnetic terms by conducting parity-violation experiments at both forward and backward angles. The forward angle asymmetry measurement is able to determine the linear combination of $G_{E}^{s}$ and $G_{M}^{s}$. Two subsequent backward angle measurements on hydrogen and deuterium can separate these two form factors and the axial form factors. The hydrogen measurement is sensitive to $G_{M}^{s}$ and $G_{A}^{e}$, while the deuterium measurement tends to enhance the sensitivity to $G_{A}^{e}$. The deuterium asymmetry, $A_{d}$, can be written 
in terms of the linear combination of the individual neutron and proton asymmetries weighed by their cross sections

$$
A_{d}=\frac{A_{Q E} \sigma_{Q E}+A_{E} \sigma_{E}}{\sigma_{Q E}+\sigma_{E}}
$$

where $A_{Q E}$ and $A_{E}$ are the asymmetries for quasi-elastic and elastic scattering of electrons from deuterium, and $\sigma_{Q E}$ and $\sigma_{E}$ are the cross sections for the same two scattering processes. As can be seen, the deuterium measurement weighs the contributions from $G_{M}^{s}$ and $G_{A}^{e}$ differently compared to the backward angle deuterium measurement. This feature of the deuterium measurement is used to separate the individual contributions of $G_{M}^{s}$ and $G_{A}^{e}$. Although the $G^{0}$ experiment will perform all three measurements (forward angle hydrogen, backward angle hydrogen and deuterium), to determine the three unknowns $\left(G_{E}^{s}, G_{M}^{s}\right.$, and $\left.G_{A}^{e}\right)$, this dissertation will focus on the forward angle measurement which was completed in 2004.

\subsubsection{Electroweak Radiative Corrections}

The asymmetry in Eqn. 1.43 comes from the tree-level interactions seen in Fig. 1.2. However, contributions from heavy quark and higher order diagrams of so called "one-quark" and "multiquark" electroweak radiative corrections must be considered.

Heavy quark effects refer to the contribution of $c, b$, and $t$ quarks which were neglected in the calculation of the hadronic currents. However, when these effects are computed, they are seen to

be small, with the vector coupling $\Delta v<10^{-4}$ and the axial coupling $\Delta A<10^{-2}[16]$. These contributions are neglected due to their small size.

One-quark corrections that do not involve knowledge of quark interactions in the non-perturbative regime can be calculated through electroweak theory, and therefore have little uncertainty. The basic formalism of these one-quark corrections can be found in [13]. These corrections have the effect of correcting the tree-level weak couplings seen in Table 1.1 by taking into account higher order diagrams. Examples of such higher order one-quark diagrams can be seen in Fig. 1.3. The numerical values of the one-quark SM parameters can be found in the Particle Data Book [14], and are calculated in the $\overline{M S}$ (modified minimal subtraction) scheme.

Multi-quark corrections involve the exchange of a photon with multiple quarks within the nu- 

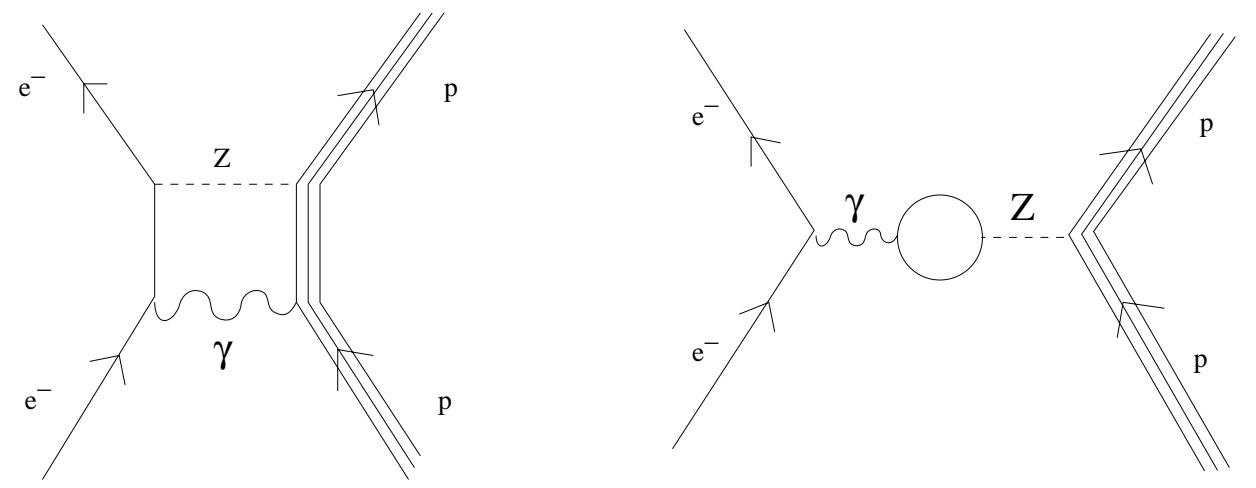

Figure 1.3: One-quark electroweak radiative corrections. On the left is a " $\gamma-\mathrm{Z}$ box", and on the right a " $\gamma$-Z mixing".

cleon interacting weakly. This weak interaction between quarks gives rise to an additional parityviolating terms called the "anapole moment". However, the interaction of quarks at distance scales of the order of the nucleon would involve non-perturbative QCD, which is not well understood. Therefore, the calculation of these multi-quark corrections involve various phenomenological hadronic models [18] [20] [21]. Some representative processes that enter the multi-quark correction are shown in Fig. 1.4. Experimental determinations of the anapole correction using parity-violation
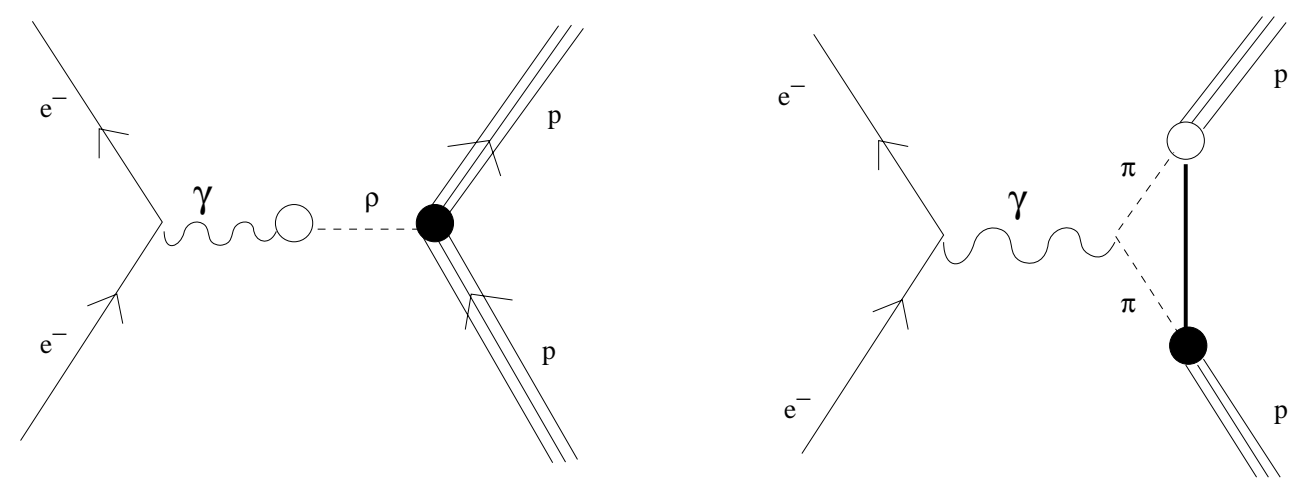

Figure 1.4: Multi-quark electroweak radiative corrections. The filled circles represent axial couplings, while the unfilled circles represent the vector couplings. The left diagram shows a Rho meson pole, while the one on the right is a pion loop diagram.

with cesium atoms [19] have shown that such higher order terms can be of the same magnitude as the tree-level $Z^{0}$ amplitude.

The contribution due to multi-quark effects modifies the electromagnetic amplitude written in 
Eqn. 1.14 by adding an anapole term to the proton current

$$
M_{\gamma}=\frac{4 \pi \alpha g_{\mu \nu}}{Q^{2}} j_{\gamma}^{\mu}\left(J_{\gamma}^{\nu}+J_{\gamma}^{\nu, \text { anapole }}\right)
$$

where $j_{\gamma}^{\mu}$ and $J_{\gamma}^{\nu}$ are the same lepton and hadron currents from Eqns. 1.15 and 1.16, and $J_{\gamma}^{\nu, \text { anapole }}$ is the anapole current [13]

$$
\left.J_{\gamma}^{\mu, \text { anapole }}=\kappa\left(\alpha_{s} F_{s}^{\text {anapole }}\left(Q^{2}\right)+\alpha_{v} F_{v}^{\text {anapole }}\left(Q^{2}\right)\right) \overline{u_{x}}\left[\left(q^{2} \gamma^{\mu}-q^{\mu} \Gamma\right) \gamma_{5}\right)\right] u_{x}
$$

where $F_{s}^{\text {anapole }}\left(Q^{2}\right)$ and $F_{v}^{\text {anapole }}\left(Q^{2}\right)$ are scalar and vector anapole form factors, $\alpha_{s}$ and $\alpha_{v}$ are the scalar and vector contributions to the anapole moment, and $\kappa$ is a numerical constant which will allow to normalize the different theoretical calculations of the anapole moment according to the lagrangians that are used. The anapole contribution to the asymmetry is folded into the axial term in Eqn. 1.43. The electroweak radiative corrections from one- and multi-quark corrections are characterized by a series of small factors, $R$, which enter in the asymmetry as follows [17]

$$
\begin{aligned}
A_{p}= & -\frac{G_{F} Q^{2}}{4 \pi \alpha \sqrt{2}}\left(\left(1-4 \sin ^{2} \theta_{w}\right)\left(1+R_{V}^{p}\right)\right) \\
& +\left(\frac{G_{F} Q^{2}}{4 \pi \alpha \sqrt{2}}\right) \frac{\left(1+R_{V}^{n}\right)\left(\epsilon G_{E}^{\gamma, p} G_{E}^{\gamma, n}+\tau G_{M}^{\gamma, p} G_{M}^{\gamma, n}\right)}{\epsilon\left(G_{E}^{\gamma, p}\right)^{2}+\tau\left(G_{M}^{\gamma, p}\right)^{2}} \\
& +\left(\frac{G_{F} Q^{2}}{4 \pi \alpha \sqrt{2}}\right) \frac{\left(1+R_{V}^{0}\right)\left(\epsilon G_{E}^{\gamma, p} G_{E}^{s}+\tau G_{M}^{\gamma, p} G_{M}^{s}\right)}{\epsilon\left(G_{E}^{\gamma, p}\right)^{2}+\tau\left(G_{M}^{\gamma, p}\right)^{2}} \\
& +\left(\frac{G_{F} Q^{2}}{4 \pi \alpha \sqrt{2}}\right) \frac{\left(1-4 \sin ^{2} \theta_{w}\right) \epsilon^{\prime} G_{M}^{\gamma, p} G_{A}^{e}}{\epsilon\left(G_{E}^{\gamma, p}\right)^{2}+\tau\left(G_{M}^{\gamma, p}\right)^{2}}
\end{aligned}
$$

where $R_{V}^{n}, R_{V}^{p}$, and $R_{V}^{0}$ come from one-quark corrections, and the axial term, $G_{A}^{e}$, now includes both the one-quark and the multi-quark radiative correction terms

$$
G_{A}^{e}=G_{A}^{p, Z}+\eta F_{A}+R_{e}
$$

where $G_{A}^{p, Z}$ is the proton axial form factor, $\eta=\frac{8 \pi \sqrt{2} \alpha}{1-4 \sin ^{2} \theta_{W}}, F_{A}$ is the nucleon anapole form factor, and $R_{e}$ is a radiative correction term. In the limit where no radiative corrections are included (i.e. $R_{V}^{n, p, 0}=0$ and $\left.F_{A}=0\right)$ one can see that Eqn. 1.48, when combined with the relation for $G_{E, M}^{s}$ 
in Eqn. 1.34, reduces to Eqn. 1.43. The separation of $F_{A}$ and $R_{e}$ in Eqn. 1.48 depends upon the choice of gauge, but they roughly represent the anapole contribution. The axial form factor, $G_{A}^{p, Z}$, is expressed in a dipole form with radiative correction terms $\left(R_{A}^{T=1}, R_{A}^{T=0}, R_{A}^{(0)}\right)$ of the axial weak charge

$$
\begin{aligned}
& G_{A}^{p, Z}=-g_{A}\left[1+R_{A}^{T=1}-R_{A}^{T=0} \frac{a_{8}}{2 a_{3}}\right] G_{A}^{D}\left(Q^{2}\right)+\left(1+R_{A}^{(0)} \Delta s G_{A}^{D}\left(Q^{2}\right)\right) \\
& G_{A}^{D}\left(Q^{2}\right)=\frac{1}{\left(1+\frac{Q^{2}}{M_{A}^{2}}\right)^{2}}
\end{aligned}
$$

where $M_{A}^{2}$ is the axial mass, and $\Delta s$ is the strangeness content of the nucleon.

The relation of the various radiative corrections terms, $R_{V, A}^{(0), T=0,1}$, to the axial and vector weak couplings can be seen below [17]

$$
\begin{aligned}
& R_{V}^{p}=\frac{2 g_{V}^{u}+g_{V}^{d}}{1-4 \sin ^{2} \theta_{w}}-1 \\
& R_{V}^{n}=-\left(1+g_{V}^{u}+2 g_{V}^{d}\right) \\
& R_{V}^{T=1}=\frac{g_{V}^{u}-g_{V}^{d}}{2\left(1-2 \sin ^{2} \theta_{w}\right)}-1 \\
& R_{V}^{T=0}=-\frac{-3\left(g_{V}^{u}+g_{V}^{d}\right)}{4 \sin ^{2} \theta_{w}}-1 \\
& R_{V}^{(0)}=-\left(1+g_{V}^{u}+g_{V}^{d}+g_{V}^{s}\right) \\
& R_{A}^{T=1}=-\frac{g_{A}^{u}-g_{A}^{d}}{2}-1 \\
& R_{A}^{T=0}=g_{A}^{u}+g_{A}^{d} \\
& R_{A}^{(0)}=g_{A}^{u}+g_{A}^{d}+g_{A}^{s}-1
\end{aligned}
$$

At tree level, the values of $g_{V, A}^{u}, g_{V, A}^{d}$, and $g_{V, A}^{s}$ are given in Table 1.1. The radiative correction terms in Eqn. 1.58 result from the deviation of these vector and axial weak charges from tree level when higher order terms are included. The SM values can be seen in Table 1.2

\begin{tabular}{|c|c|c|}
\hline Particle & $g_{V}^{Z}$ & $g_{A}^{Z}$ \\
\hline $\mathrm{u}, \mathrm{c}, \mathrm{t}$ & 0.377296 & -0.953567 \\
\hline $\mathrm{d}, \mathrm{s}, \mathrm{b}$ & -0.682753 & 0.700907 \\
\hline
\end{tabular}

Table 1.2: Values of vector and axial weak charges. 
The numerical values of various correction factors and constants that enter into Eqn. 1.48 and 1.50 can be seen in Table 1.3.

\begin{tabular}{|c|c|}
\hline Term & Correction \\
\hline$R_{V}^{p}$ & -0.0447091 \\
\hline$R_{V}^{n}$ & -0.0117890 \\
\hline$R_{V}^{T=1}$ & -0.0140914 \\
\hline$R_{V}^{T=0}$ & -0.0091121 \\
\hline$R_{V}^{(0)}$ & -0.0117890 \\
\hline$R_{A}^{T=1}$ & -0.1727633 \\
\hline$R_{A}^{T=0}$ & -0.2526596 \\
\hline$R_{A}^{(0)}$ & -0.5517526 \\
\hline$a_{3}$ & $1.267 \pm 0.035$ \\
\hline$a_{8}$ & $0.585 \pm 0.025$ \\
\hline$\Delta \mathrm{s}$ & -0.084 \\
\hline$M_{A}$ & $1.00 \pm 0.02\left(\mathrm{GeV} / \mathrm{c}^{2}\right)$ \\
\hline$\alpha$ & $7.29735 \times 10^{-3}$ \\
\hline$M_{p}$ & $938.272 \times 10^{-3}\left(\mathrm{GeV} / \mathrm{c}^{2}\right)$ \\
\hline$\mu_{p}$ & 2.792847351 \\
\hline$\mu_{n}$ & -1.9130427 \\
\hline $\sin ^{2} \theta_{w}$ & 0.23120 \\
\hline$G_{F}$ & $1.16639 \times 10^{-5}$ \\
\hline
\end{tabular}

Table 1.3: Terms involved in electroweak radiative corrections. These terms can be found in the Particle Data Book [14].

As can be seen in Eqn. 1.48, the asymmetry written with the electroweak radiative corrections are dependent on the strange form factors. The asymmetry calculated with $G_{E}^{s}=G_{M}^{s}=0$ is called the "no-vector-strange" asymmetry, $A_{N V S}$ where strange quarks do not contribute to proton structure. The asymmetry measured in the $G^{0}$ experiment can be compared to $A_{N V S}$ to see the degree to which strange quarks contribute to the proton's charge and magnetization distribution.

\subsubsection{Theoretical Predictions of $G_{E, M}^{s}$}

A full non-perturbative QCD calculation of $G_{E, M}^{s}$ remains very difficult to this day. However, attempts have been made to quantify these values at $Q^{2}=0$, corresponding to the strangeness radius, $r_{s}$, and the strange magnetic moment, $\mu_{s}$. Most theoretical models concentrate on the calculation of $\mu_{s}$, whereas some also attempt to determine $r_{s}$. There have been various attempts to determine the strange magnetic moment of the proton through various theoretical models. The models used 
can roughly be separated into two groups. One is the hadronic model, which employs an intuitive phenomenological approach to modeling the strangeness content within the nucleon. The other is a more first-principle approach of describing the existence of the $s \bar{s}$ pair through gluon splitting. This section will provide an overview of the various attempts at quantifying $\mu_{s}$.

\section{Hadronic models}

A variety of hadronic models have been used to calculate $\mu_{s}$ to date. These models make use of strange intermediate hadronic states to approximate the strangeness content of the proton. Hadronic models that make use of loops and poles will be discussed here. A phenomenological view of the two types of models can be seen in Fig. 1.5.
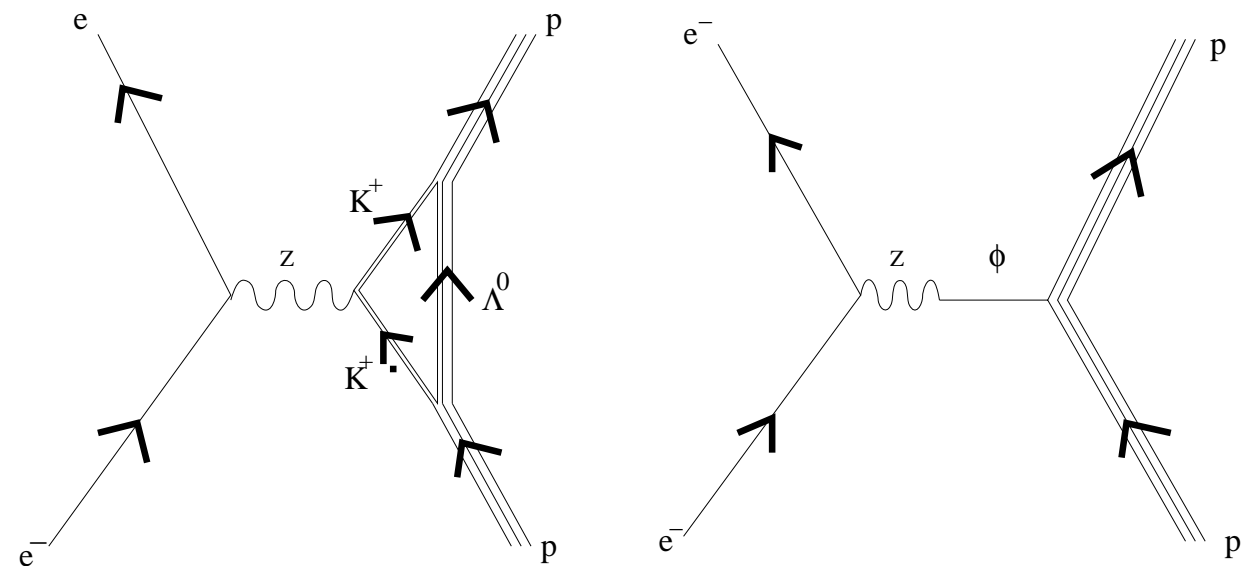

Figure 1.5: Examples of hadronic models used to compute the strangeness content of the proton. They are the "loop" (left) and "pole" (right) models.

The loop model is often used in the context of chiral perturbation theory (CHPT), where the QCD lagrangian possess an exact $S U(3)_{L} \times S U(3)_{R}$ symmetry in the limit where the light quark masses vanish [22]. The basic strategy of CHPT is to exploit this chiral symmetry to relate one set of observables to another, or to draw on one set of measured quantities to predict another [23]. However, this framework breaks down in the flavor singlet $\left(G^{0}\right)$ channel, since shortdistance hadronic effects cannot be determined from existing measurement using chiral symmetry. Although the long-distance contributions are calculable, there is no reason to believe that they are the dominant effects over the chiral counter-terms that arise at the same or lower order. Therefore, to determine the strangeness contribution requires the a-priori knowledge of the strangeness radius 
and magnetic moment. The theory, thus, relies on experiment to supply these quantities, and has no predictive power of the strange quark contribution to proton structure [24]. In order to make predictions for the nucleon's strangeness moments, model dependent assumptions must be invoked to go beyond the framework of CHPT.

One such model is the kaon loop model (or kaon cloud dominance), which abandons the requirement of a consistent chiral expansion, and includes a kaon loop contribution [25] [27] [28] [29]. There are two main motivations for choosing such a model. The first comes from a geometric interpretation of the nucleon charge radius, where the model characterizes a spatial asymmetry in the charge distribution of $s$ and $\bar{s}$. In the loop model, the strangeness of the proton is described through a strange meson-hyperon intermediate state with which either the exchange photon or Z interacts. Typically, the proton is modeled to fluctuate into the lightest strange meson, a kaon, and a $\Lambda$ hyperon. The $s \bar{s}$ quarks then annihilate to leave the original proton. Shown in Fig. 1.5 is a proton fluctuating into a $K^{+} \Lambda^{0}$ intermediate state. This intermediate state allows for the $s$ and $\bar{s}$ to spatially separate due to the differing masses of the two intermediate state particles. The result is an asymmetric and non-zero strange charge and magnetization distribution within the proton. The second motivation comes from the success of the pion loop model in calculating the nucleon's electromagnetic form factors by Bethe and DeHoffman [30]. In this model, the pion cloud dominates the nucleon's isovector electromagnetic moments, and a one loop calculation sufficiently incorporates the physics of the pion cloud. At the time this was reported ${ }^{2}$, the results were in surprising agreement with the experimental values seen for the nucleon's charge radius. If this situation were to persist in the strangeness sector, a kaon cloud may give the dominant contribution to the strangeness moments, and a one-loop calculation would suffice to give their correct magnitude and sign.

An example of a one-loop calculation is one made by Musolf and Burkardt [25], which uses a nonlinear $\mathrm{SU}(3) \sigma$ model, and introduces a form factor at the hadronic vertices to ensure the desired strangeness radius is not UV divergent. They chose a simple monopole form for the hadronic form factor

$$
F(k)=\left(\frac{m_{K}^{2}-\Lambda^{2}}{k^{2}-\Lambda^{2}}\right)
$$

\footnotetext{
${ }^{2}$ Later studies by Drell and Pagels [31] showed that the hadronic expansion does not converge for the calculation of the anomalous moments of the proton.
} 
to include in the chiral loop integrals involving baryons, where $m_{K}$ is the kaon mass, $\Lambda^{2}$ is the momentum cutoff of the loop integration, and $k$ is the momentum of the kaon at the KN $\Lambda$ vertex. Various kaon cloud models differ, in part, through the choice of form for $F(k)$ and the value of the cutoff parameter. For example, Donoghue and Holstein [26] chose a dipole form

$$
F\left(q^{2}\right)=\left(\frac{\Lambda^{2}}{\Lambda^{2}-q^{2}}\right)^{2}
$$

and showed that this retains the underlying chiral structure. Furthermore, the introduction of these form factors necessitates the introduction of "seagull" diagrams in the loop integrals to preserve the Ward-Takahashi identities so as to ensure gauge invariance.

Another loop calculation is one by Koepf, Henley and Pollock [27], which uses the cloudy bag model $(\mathrm{CBM})$ in order to estimate the strangeness moments. The CBM is similar to the MIT bag model, where the valence quarks are allowed to exist inside "bags" of perturbative vacuum. However, the MIT bag model does not maintain chiral symmetry. The CBM model improves upon this by including the interaction of the confined quarks with the meson fields on the bag surface, thereby imposing chiral symmetry. The resulting strange matrix element is similar to the simple kaon loop picture. Several other attempts to make use of a kaon loop picture are noted in [24].

In the pole model [32] [33], as opposed to the loop model, the strangeness comes when the exchange photon fluctuates into a strange meson. The basic approach is to model the spectral functions of the isoscalar form factors via a dispersion relation calculation. The $F_{1}^{T=0}$ and $F_{2}^{T=0}$ $\left(F_{i}^{T=0}=\frac{F_{i}^{p}-F_{i}^{n}}{2}, \mathrm{i}=1,2\right)$ form factors, which are measured through experiment, are fitted with a linear combination of vector meson poles

$$
\begin{aligned}
& F_{1}^{T=0}\left(q^{2}\right)=F_{1}^{T=0}+\Sigma_{V} \frac{q^{2} a_{V}^{T=0}}{m_{V}^{2}-q^{2}} \\
& F_{2}^{T=0}\left(q^{2}\right)=\Sigma_{V} \frac{m_{V}^{2} b_{V}^{T=0}}{m_{V}^{2}-q^{2}}
\end{aligned}
$$

where $m_{V}$ is the mass of the vector meson in question. The vector mesons used for the fit involved the $\omega$ and $\phi$ mesons as well as a higher mass vector meson $V^{\prime}$. At least two poles are needed to obtain a dipole dependence of the form factors, while a third pole is included to achieve a reasonable $\chi^{2}$ for the fit. 
Numerous other models have been tried to calculate the strangeness moment of the nucleon, and their results are shown in Table 1.4. The constituent quarks model treats the nucleon's strangeness matrix elements as arising from the strangeness "content" of constituent U and D quarks. Constituent quark strangeness current operators are derived, and the matrix elements are computed from those operators using the appropriate bound state wave function. The Nambu-Jona-Lasinio (NJL) model [34] uses such an approach to compute the constituent quark strangeness radii. Another alternative is to adopt a chiral quark model [24] [35], where the constituent quark strangeness currents arise from fluctuations of constituent $\mathrm{U}$ and $\mathrm{D}$ quarks into a kaon plus a constituent $\mathrm{S}$ quark. The contributions from the individual $\mathrm{U}$ and $\mathrm{D}$ quarks are added to give the total nucleon strangeness matrix element using a quark model spin-space-flavor wave function. Although this model does not suffer from the lack of convergence that is seen in traditional HBCHPT, the ambiguities associated with the chiral counter-terms remain.

\section{Lattice QCD}

Apart from the various hadronic models used to calculate the strangeness moment, there are first principle approaches to determining $\mu_{s}$, such as lattice QCD (LQCD). LQCD is a non-perturbative computational method, where QCD is applied on a lattice of space-time points. Although this may be less susceptible to the model dependence seen in the various hadronic models, the application of LQCD to computing the contributions from $q \bar{q}$ loops and using quarks with their physical masses is too computationally expensive. Therefore, many of the LQCD methods use the quenched approximation, where quarks are assumed not to be dynamical. The various calculations within LQCD have resulted in values of $\mu_{s}$ ranging from $-0.36 \pm 0.20$ [36] to $0.04 \pm 0.04$ [37]. One of the most recent LQCD calculations by Leinweber et al attempt to improve upon this situation by combining the constraints of charge symmetry and new chiral extrapolation techniques, to account for the difference between the quark masses in the calculation and their physical values, with quenched LQCD simulations to determine a strangeness moment of $-0.046 \pm 0.019$ n.m [38]. However, this result, as will be shown, is inconsistent with the most recent experimental results, including those from the $G^{0}$ forward angle measurement. 


\section{Summary}

There are a number of other approaches to calculating $\mu_{s}$ which differ in detail from those described above, some of which are shown in Table 1.4. As can be seen, there is some variation to the re-

\begin{tabular}{|c|c|}
\hline Type of calculation & $\mu_{s}$ (n.m.) \\
\hline Poles [32] & $-0.31 \pm 0.09$ \\
\hline Kaon Loops [25] & $-0.31 \rightarrow-0.40$ \\
\hline Kaon Loops [27] & -0.026 \\
\hline Loops [28] & 0.035 \\
\hline SU(3) Skyrme (broken) [39] & -0.13 \\
\hline SU(3) chiral hyperbag [40] & 0.42 \\
\hline NJL soliton [34] & $-0.05 \rightarrow 0.25$ \\
\hline QCD equalities [41] & $-0.75 \pm 0.30$ \\
\hline Dispersion relations [42] & $-0.10 \rightarrow-0.14$ \\
\hline Chiral quark [35] & -0.04 \\
\hline Lattice QCD [38] & $-0.46 \pm 0.019$ \\
\hline
\end{tabular}

Table 1.4: Theoretical predictions of $\mu_{s}$.

sults obtained from the differing models, signifying the difficulty of the task. Overall, however, the predictions seem to indicate a preference for a negative value of $\mu_{s}$. As will be shown in the next section, this is in contradiction with the experimental determinations which show that $\mu_{s}$ seems to be positive at low $Q^{2}$.

\subsubsection{Previous Experiments}

While the apparent difficulty of creating a consistent model for calculating $\mu_{s}$ has produced a multitude of theoretical predictions, experimental efforts were underway to empirically measure this value. Parity-violating experiments have been performed at MIT-Bates, at MAMI, and at Jefferson Lab, and have produced consistent determinations of $G_{M}^{s}$ at low $Q^{2}$. This section will focus on previous experimental efforts to determine the strange form factors of the proton.

\section{The SAMPLE Experiment}

The first experiment that measured the strange magnetic form factor of the proton was the SAMPLE experiment, conducted at MIT-BATES [43] [44]. A $40 \mu \mathrm{A}$ longitudinally polarized electron beam was incident on a liquid hydrogen target. The SAMPLE experiment measured PV asym- 
metries at backward angles by detecting back-scattered electrons with an array of air Cerenkov detectors (Fig. 1.6). Initial measurements were taken at a beam energy of $200 \mathrm{MeV}$ and a $Q^{2}$ of

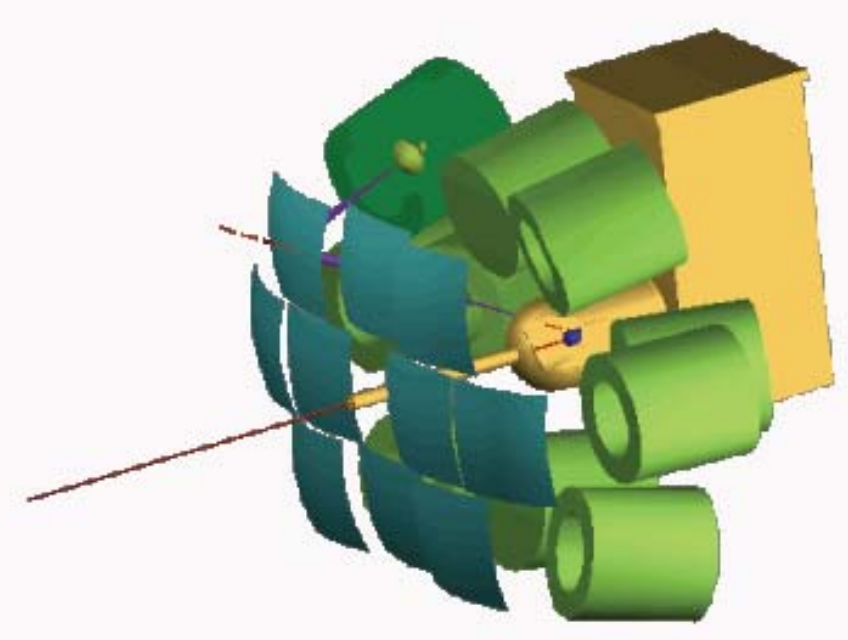

Figure 1.6: Schematic of the SAMPLE experiment. Air Cherenkov detectors at a scattering angle of about $147^{\circ}$ detect the backscattered electrons, whose Cherenkov light is reflected off of a set of ellipsoidal mirrors facing ten PMTs. The signals in each PMT are then integrated.

$0.1(\mathrm{GeV} / \mathrm{c})^{2}$. This measurement taken in the summer of 1998, was on a liquid hydrogen target. The second, in the summer of 1999, was on a liquid deuterium target. A final measurement was taken during the winter of 2001-2002 with a beam energy of $125 \mathrm{MeV}$ on a liquid deuterium target. As discussed previously, at backward angles, the asymmetry is sensitive to the linear combination of $G_{M}^{s}$ and $G_{A}^{e}$. The strange quark contribution to the proton magnetic form factor and the isovector portion of the axial form factor can be determined by combining the proton and deuteron asymmetries

$$
\begin{aligned}
& G_{M}^{s}\left(Q^{2}=0.1\right)=0.28 \pm 0.45 \pm 0.39 \pm 0.03 n . m . \\
& G_{A}^{e}(T=1)=-0.66 \pm 0.57 \pm 0.50 \pm 0.12
\end{aligned}
$$

where the uncertainties are statistical, systematic, and electroweak radiative corrections, respectively. These results can be compared with the theoretical predictions for the strange magnetic 
moment, although there are uncertainties associated with the extrapolation of the results to zero momentum transfer, since the $Q^{2}$ dependence of $G_{M}^{s}$ is not known. A prediction based on heavy baryon chiral perturbation theory indicates the $Q^{2}$ extrapolation could shift $G_{M}^{s}\left(Q^{2}=0.1\right)$ by about 0.2 n.m in either direction. Therefore, the extrapolated value of $\mu_{s}$ would have similar magnitude compared to many of the theoretical models, but there seems to be a disagreement in the sign between theory and experiment.

\section{The HAPPEX Experiment}

The HAPPEX experiment [45] [46] [47], which runs at Jefferson Lab, measures PV asymmetries at forward scattering angles. Scattered electrons are focused by two high-resolution spectrometers onto lead-lucite Cherenkov detectors. The signals from the detectors are collected by PMTs and integrated in order to accommodate high counting rates. A schematic of the experiment from the source to the detectors can be seen in Fig. 1.7.

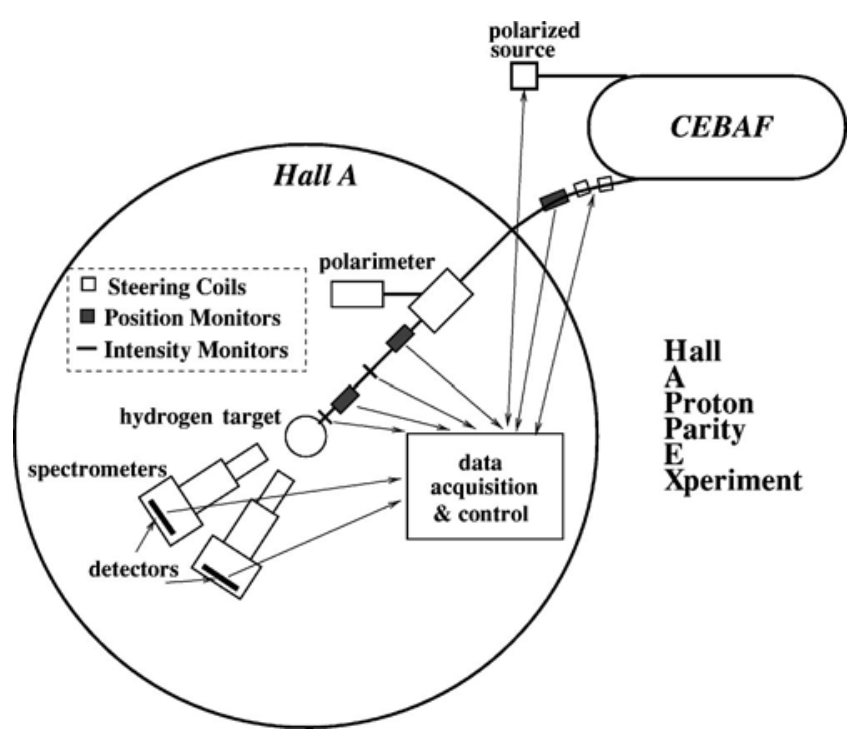

Figure 1.7: Schematic of the HAPPEX experiment. The electron beam from the source scatters off the target, and are detected by a set of lead-lucite Cherenkov detectors.

To date, the experiment has made three measurements. The first experimental data, taken in two separate runs in 1998 and 1999 in Hall A, measured the PV asymmetry at an electron scattering angle of $\left\langle\theta_{e}\right\rangle=12.3^{\circ}$ and a beam energy of $3.2 \mathrm{GeV}$. This corresponds to a $Q^{2}$ of $0.477(\mathrm{GeV} / \mathrm{c})^{2}$. 
At forward angles, the asymmetry is sensitive to the linear combination of $G_{E}^{s}$ and $G_{M}^{s}$

$$
\left(G_{E}^{s}+0.392 G_{M}^{s}\right)\left(Q^{2}=0.477\right)=0.014 \pm 0.020 \pm 0.010
$$

The result is consistent with zero. However, this does not rule out the possibility that $G_{E}^{s}$ and $G_{M}^{s}$ are non-zero and may be canceling each other out. A backward angle measurement at this kinematic point will be necessary to separate the two strange form factors.

The other two HAPPEX measurements were taken in 2004 on a liquid hydrogen and a ${ }^{4} \mathrm{He}$ target. The hydrogen measurement was at a forward electron scattering angle of $\left\langle\theta_{e}\right\rangle=6.0^{\circ}$ and a beam energy of $3.03 \mathrm{GeV}$. The ${ }^{4} \mathrm{He}$ measurement was at $\left\langle\theta_{e}\right\rangle=5.7^{\circ}$ and the same beam energy as for the hydrogen measurement. These kinematics correspond to a $Q^{2}$ of $0.099(\mathrm{GeV} / \mathrm{c})^{2}$ and $0.091(\mathrm{GeV} / \mathrm{c})^{2}$, respectively. Although the ${ }^{4} \mathrm{He}$ measurement was taken at forward angles, the asymmetry for ${ }^{4}$ He scattering turns out to be insensitive to $G_{M}^{s}$, and therefore allows for the direct measurement of $G_{E}^{s}$

$$
\begin{aligned}
& \left(G_{E}^{s}+0.080 G_{M}^{s}\right)\left(Q^{2}=0.099\right)(H)=0.030 \pm 0.025 \pm 0.006 \pm 0.012 \\
& \left(G_{E}^{s}\right)\left(Q^{2}=0.091\right)\left({ }^{4} H e\right)=-0.038 \pm 0.042 \pm 0.010
\end{aligned}
$$

The errors on the hydrogen measurement are statistical, systematic, and the error arising from the neutron electromagnetic form factor, respectively. The two errors from the ${ }^{4} \mathrm{He}$ measurement are the statistical and systematic uncertainties. The advantage of measuring both on ${ }^{4} \mathrm{He}$ and hydrogen is that this allows for the complete separation of $G_{E}^{s}$ and $G_{M}^{s}$. Furthermore, it is possible to combine the above measurements with the backward angle SAMPLE results, which is very close in $Q^{2}$ to the HAPPEX $\mathrm{H}$ and ${ }^{4} \mathrm{He}$ measurements, thereby improving the statistics on the final separation. The two measurements on hydrogen and ${ }^{4} \mathrm{He}$ at $Q^{2} \approx 0.1(\mathrm{GeV} / \mathrm{c})^{2}$ are on-going. The results above reflect only a fraction of the total statistics that the HAPPEX collaboration expects to achieve. The future developments of the HAPPEX experiment will be discussed later in the conclusions section. 


\section{The PVA4 Experiment}

The PVA4 experiment [48] [49] takes place at MAMI in Mainz, scattering longitudinally polarized electrons off unpolarized protons. The experiment detects forward scattered electrons with a total absorption calorimeter that consists of 1022 individual lead fluoride crystals placed in 7 rings and 146 rows. This covers a range of scattering angles from $30^{\circ}<\theta_{e}<40^{\circ}$, and a full $360^{\circ}$ azimuthal range, although only half of this range, or 511 detector channels, were operational for the initial measurement. A schematic of the PVA4 detector can be seen in Fig. 1.8.

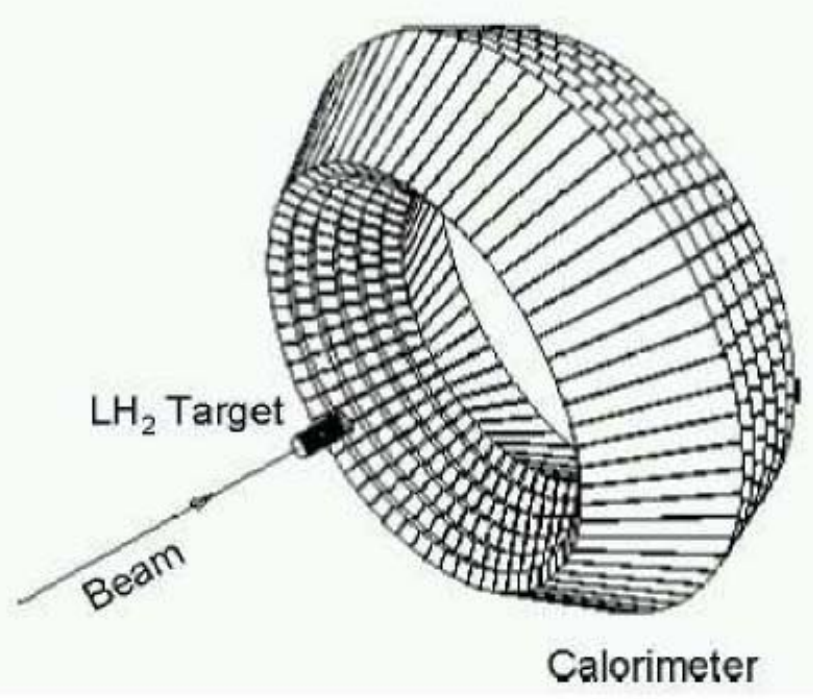

Figure 1.8: Schematic of the PVA4 calorimeter. The electrons are detected by an array of lead fluoride crystals.

The PVA4 experiment has so far made two measurements of the PV asymmetry; one at a $Q^{2}$ $=0.230(\mathrm{GeV} / \mathrm{c})^{2}$ with half of the detectors operational, and another at $0.108(\mathrm{GeV} / \mathrm{c})^{2}$ with all 1022 detectors and full azimuthal range. The forward angle results measure a linear combination of $G_{E}^{s}$ and $G_{M}^{s}$ at both $Q^{2}$

$$
\begin{aligned}
& \left(G_{E}^{s}+0.106 G_{M}^{s}\right)\left(Q^{2}=0.108\right)=0.071 \pm 0.036 \\
& \left(G_{E}^{s}+0.225 G_{M}^{s}\right)\left(Q^{2}=0.230\right)=0.039 \pm 0.034
\end{aligned}
$$


As with the HAPPEX results, the PVA4 results at $Q^{2}=0.1$ can be combined with previous measurements to constrain both $G_{E}^{s}$ and $G_{M}^{s}$ at this $Q^{2}$.

\section{Summary of Previous Experiments}

The various PV experiments conducted to date have concentrated on $Q^{2} \approx 0.1(\mathrm{GeV} / \mathrm{c})^{2}$. The results of these experiments can be seen in Fig. 1.9, shown in a $G_{E}^{s}$ vs $G_{M}^{s}$ plot. All experiments

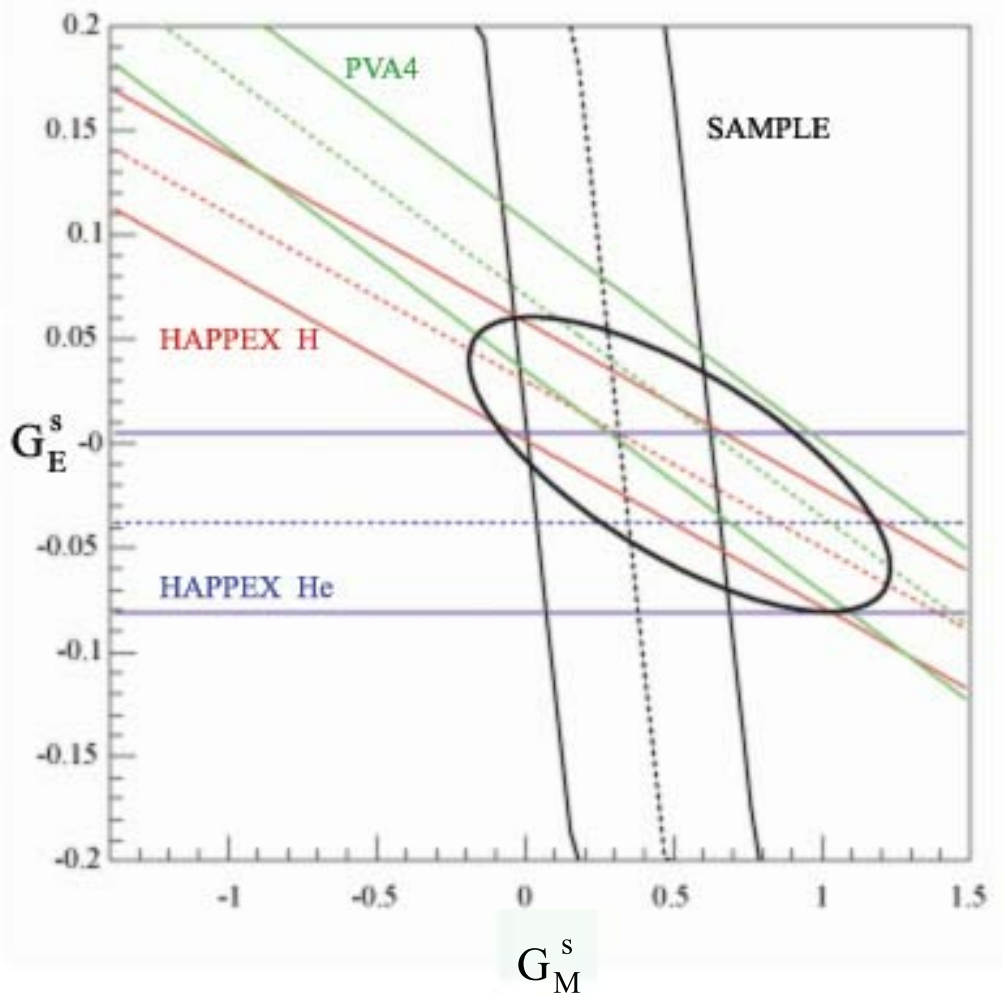

Figure 1.9: Summary of previous experiments conducted at $Q^{2}=0.1(G e V / c)^{2}$. The different slopes for each experiment results from the differing angular kinematics and beam energy of each experiment. The ellipse represents a $95.5 \%$ confidence interval with central values of $G_{E}^{s}=-0.01$ \pm 0.03 and $G_{M}^{s}=0.55 \pm 0.28$.

conducted to date at this $Q^{2}$ show consistent results, and favor a positive value for $G_{M}^{s}$. As previous discussed, the extrapolation of $G_{M}^{s}$ to the static limit of $Q^{2}=0$ involves some uncertainty, and requires knowledge of its $Q^{2}$ dependence. However, even with this uncertainty, inconsistencies exist between experiment and theory. 
Only a few of the experiments discussed here have taken measurements at higher $Q^{2}$. The $G^{0}$ experiment, as will be discussed in greater detail in subsequent sections, is able to measure the strange quark contributions to the proton form factors over a range of $0.1<Q^{2}<1.0(\mathrm{GeV} / \mathrm{c})^{2}$, separated into 18 individual $Q^{2}$ bins. The forward angle results of the $G^{0}$ experiment shows a clear, non-trivial $Q^{2}$ dependence of the linear combination of $G_{E}^{s}$ and $G_{M}^{s}$. 


\section{Chapter 2}

\section{G0 Experimental Apparatus}

The $G^{0}$ experiment [50] [51], unlike other parity-violation experiments, attempts to separate the strange electric and magnetic form factors over a range of $Q^{2}$. In order to accomplish this, a forward angle measurement, detecting recoiled elastic protons at angles ranging from $52^{\circ}$ to $76^{\circ}$, is made to determine the linear combination of the two form factors over the range of $0.12<$ $Q^{2}<1.0(\mathrm{GeV} / \mathrm{c})^{2}$. The beam energy for the experiment is $3.028 \mathrm{GeV}$ at $40 \mu \mathrm{A}$, with a target luminosity of $2.1 \times 10^{18} \mathrm{~cm}^{-1} \mathrm{~s}^{-1}$ and a total acceptance of $0.9 \mathrm{sr}$. Subsequent backward angle runs are needed for each $Q^{2}$ for which we wish to separate the two form factors. At backward angles, we detect back-scattered elastic and quasi-elastic electrons, using both a hydrogen and a deuterium target, respectively. The parity-violating asymmetries are determined by measuring the cross section of elastic electron-proton scattering for positive and negative helicity incident electron beams. Therefore, the asymmetry can be written as

$$
A_{P V}=\frac{\sigma_{+}-\sigma_{-}}{\sigma_{+}+\sigma_{-}}
$$

where $\sigma_{+(-)}$is the elastic cross section for positive(negative) helicity states. Experimentally, the cross section is simply the elastic proton rate measured in the detectors. However, the asymmetry is constructed from the "normalized yield" which is the rate normalized to the beam current, since the rate can vary linearly with the intensity of the beam. Therefore, Eqn. 2.1 can also be written as

$$
A_{P V}=\frac{Y_{+}-Y_{-}}{Y_{+}+Y_{-}}
$$


where $Y_{+(-)}$is the normalized yield seen in the detector for the positive(negative) state. The asymmetries measured in the forward angle phase of the experiment range from $10^{-5}$ to $10^{-6}$. The goal is to measure these asymmetries with overall uncertainties of $5 \%$ to $10 \%$ of the physics asymmetry, which means systematic uncertainties must be carefully controlled to levels of $10^{-6}$ to $10^{-7}$. The sources and the methods employed to reduce these systematic uncertainties will be discussed in the analysis section.

The $G^{0}$ experiment takes place in Hall C of Jefferson Lab [52] located in Newport News, VA. A visualization of the $G^{0}$ experiment can be seen in Fig. 2.1. The apparatus involved in the experiment is the JLab accelerator, a superconducting toroidal magnet, a high power target system, and a timeencoding electronics/detector system capable of time of flight (tof) particle identification. A $40 \mu \mathrm{A}$ longitudinally polarized electron beam is delivered from the JLab accelerator, and is incident on a liquid hydrogen target. In the forward angle mode, the parity-violating asymmetry is determined by detecting the recoil protons with an array of 16 scintillator detectors placed on the focal surface of the magnet, allowing for $Q^{2}$ separation using individual scintillator segments of the detector array. Therefore, each of the 16 detectors measures an asymmetry for a particular $Q^{2}$. The exceptions are detectors 14,15 , and 16. Detector 14 has two elastic proton peaks. Detector 15 has a relatively large kinematic range and is thus binned into three separate $Q^{2}$ values. Detector 16 is outside the elastic proton acceptance, and is used to measure the background. Therefore, the $G^{0}$ forward angle experiment measures asymmetries for 18 separate $Q^{2}$ values. The electronics separates the slow-moving protons from the fast moving background such as pions and gammas using tof. This chapter will focus on providing a more detailed description of the apparatus as well as the overall performance of each of the experimental subsystems by following the path of the data from the source of the electron beam to the elastic proton tof determination in the electronics.

The experimental apparatus was installed during the summer-fall of 2002 and tested during a winter 2002-2003 commissioning run as well as during a subsequent second engineering run of winter 2003-2004. The forward angle production data were accumulated during the spring of 2004. 


\section{Go Experiment}

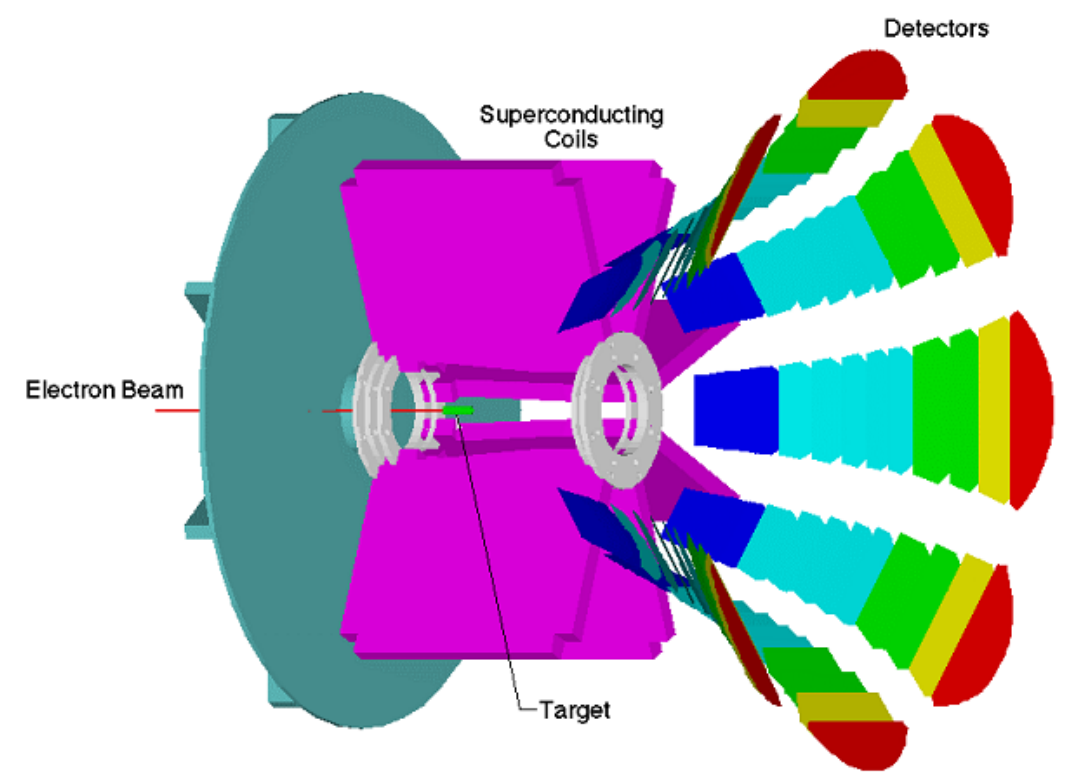

Figure 2.1: The $G^{0}$ spectrometer.

\subsection{The $G^{0}$ Polarized Electron Beam}

The experimental site consists of two linear accelerators (linacs) capable of delivering beam to three experimental halls (Halls A, B, and C) simultaneously. A schematic of the JLab accelerator can be seen in Fig. 2.2.

The accelerator is roughly composed of the polarized source where the beam originates, the injector section where the beam is bunched before acceleration, two linear accelerators (north and south linacs), the return arcs where the beam is recycled into the linacs for multiple acceleration, and the beam switch-yard where the beam is steered into one of the three halls.

\section{Polarized Source}

The JLab polarized source delivers electron beam that is longitudinally polarized up to 85\% [53]. This is achieved via the photo-electric effect by using a circularly polarized laser incident on a strained GaAs cathode [54] ${ }^{1}$. A schematic of the injector source table can be seen in Fig. 2.3. The

\footnotetext{
${ }^{1}$ Recent developments in polarized source have resulted in the use of a "super-lattice" photo-cathode, which can deliver higher polarization than the strained GaAs cathode.
} 


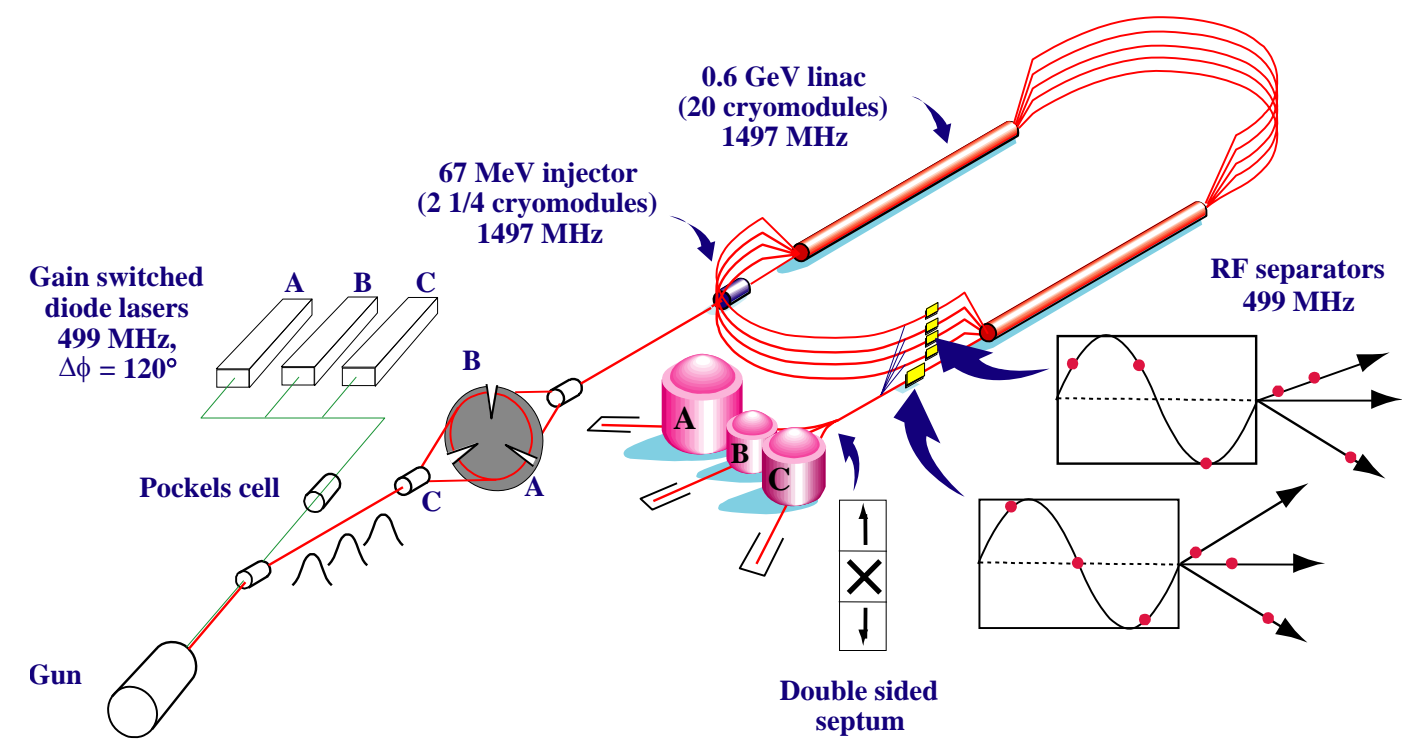

Figure 2.2: A schematic of the JLab accelerator from the source to the three halls.

circularly polarized light for Halls A and C originate from a commercially available Ti-Sapphire laser developed by Time Bandwidth Inc [55]. This Ti-Sapphire laser is preferred, over the pulsed diode laser used previously at JLab to produce $499 \mathrm{MHz}$ repetition rate beams, for its higher power and faster shut-off time [58]. The faster shut-off time for the Hall A laser in particular is a crucial improvement needed for $G^{0}$, as it has the effect of reducing the amount of beam current leaking from Hall A into Hall C. The Hall B beam originates from a diode laser. Although this laser has a slower shut-off time, Hall B typically receives beam currents of order 10's to 100's of nA (whereas Halls A and C typically receive anywhere from 10's up to $100 \mu \mathrm{A}$ ). The leakage due to this small beam current is of the same order as that from Hall A. The effect of leakage current from Halls A and B into Hall $\mathrm{C}$ will be discussed in the analysis section.

Since both the quantum efficiency $(Q E)$ and the beam polarization depends on the wavelength of the laser incident upon the cathode, the wavelength must be chosen to maximize the figure of 


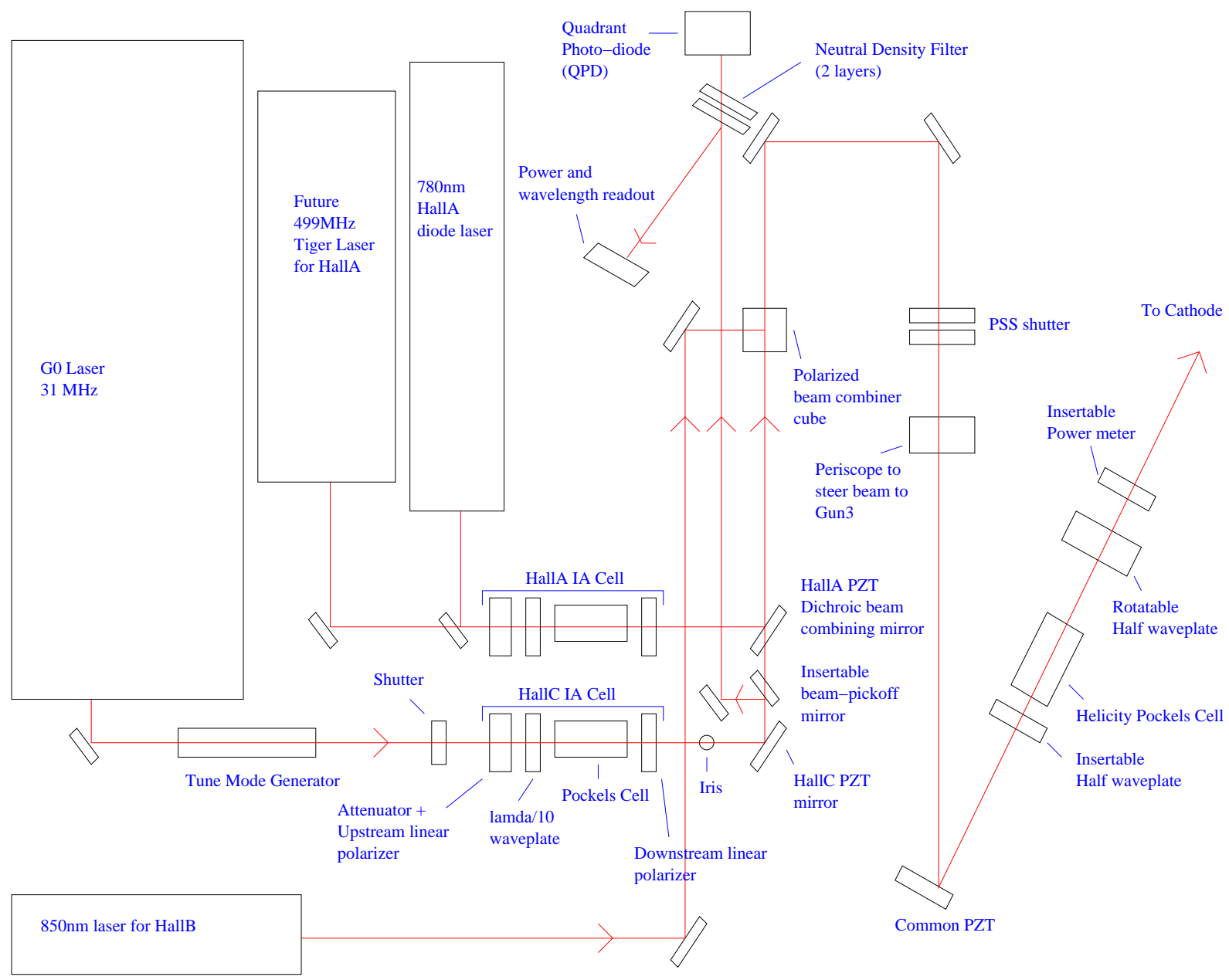

Figure 2.3: A schematic of the injector laser table setup

merit [57]

$$
\text { Figure of merito } \propto E \times P^{2}
$$

where $Q E$ is the quantum efficiency, and $P$ is the polarization ${ }^{2}$. The wavelength of the laser was consequently chosen to be $848 \mathrm{~nm}$. This amounts to a $Q E$ of about $0.2 \%$ and a polarization of about $75 \%$.

The laser incident on the cathode must be circularly polarized for the cathode to emit polarized electrons. In order to produce left and right circularly polarized light, the laser is passed through a helicity Pockels cell (HPC) with a quarter-wave voltage applied to it. The active material in

\footnotetext{
${ }^{2}$ The available beam current is proportional to the $Q E$, and the statistics on parity-violating e-p scattering is diluted as $P^{2}$.
} 
the Pockels cell is the birefringent crystal $\mathrm{KD}^{*} \mathrm{P}$, whose indices of refraction $\left(n_{x}\right.$ and $\left.n_{y}\right)$ along perpendicular axes can be changed by the application of an electric field [59]. The axis with the higher index of refraction is called the slow axis, whereas the one with the lower index is the fast axis. When linearly polarized light which, oriented $45^{\circ}$ from these axes, is passed through the crystal, the light acquires a $\frac{\pi}{2}$ phase shift, causing it to become circularly polarized. The handedness of the circular polarization can be changed by reversing the voltage across the cell. In this way, a Pockels cell can act as a variable phase retarder, which is more precisely tunable to give a higher circular polarization than a quarter-wave plate. Typical circular polarization achieved at the injector laser table was $99.98 \%$.

The circularly polarized light is incident on a strained GaAs crystal which can emit electrons with a theoretical maximum beam polarization of $100 \%$ compared to the conventional bulk GaAs crystal which can in principle yield up to $50 \%$ beam polarization. Fig. 2.4 shows a comparison of the $P_{\frac{3}{2}}$ valence and $S_{\frac{1}{2}}$ conduction bands of the two crystals. The reason for the $50 \%$ polarization limitation on the bulk crystal comes from the fact that the energy difference between the valence and conduction bands are degenerate (Fig. 2.4 a) for the left and right circularly polarized excitations [60]. The Clebsch-Gordon Coefficients for this process favor the $m_{j}=\frac{-3}{2} \rightarrow \frac{1}{2}$ and $m_{j}=\frac{3}{2} \rightarrow \frac{-1}{2}$ transitions over the $m_{j}=\frac{-1}{2} \rightarrow \frac{1}{2}$ and $m_{j}=\frac{1}{2} \rightarrow \frac{-1}{2}$ transitions by a factor of 3 . Therefore, in the case of a left circularly polarized excitation, as shown in the figure, the relative strengths of the left and right handed transitions is 3:1. The maximum polarization achievable is thus $50 \%$.

When a biaxial-compressive stress is applied by growing the GaAs on GaAsP, the degeneracy of the valence states is broken (Fig. $2.4 \mathrm{~b}$ ). Tuning the wavelength of the laser to match the new band gap results in a theoretical maximum polarization of $100 \%$. However, in practice, the achieved polarization is typically $<80 \%$ due to limited band splitting and relaxations in the strain of the crystal. Further de-polarization occurs depending on the depth below the surface of the cathode at which the electrons originate (since the electrons have a non-zero probability of flipping sign as they scatter to the surface) [60] [61].

\section{Beam Polarization}

The polarized electron beam from the source must be longitudinally polarized when it reaches the 
a. Bulk GaAs
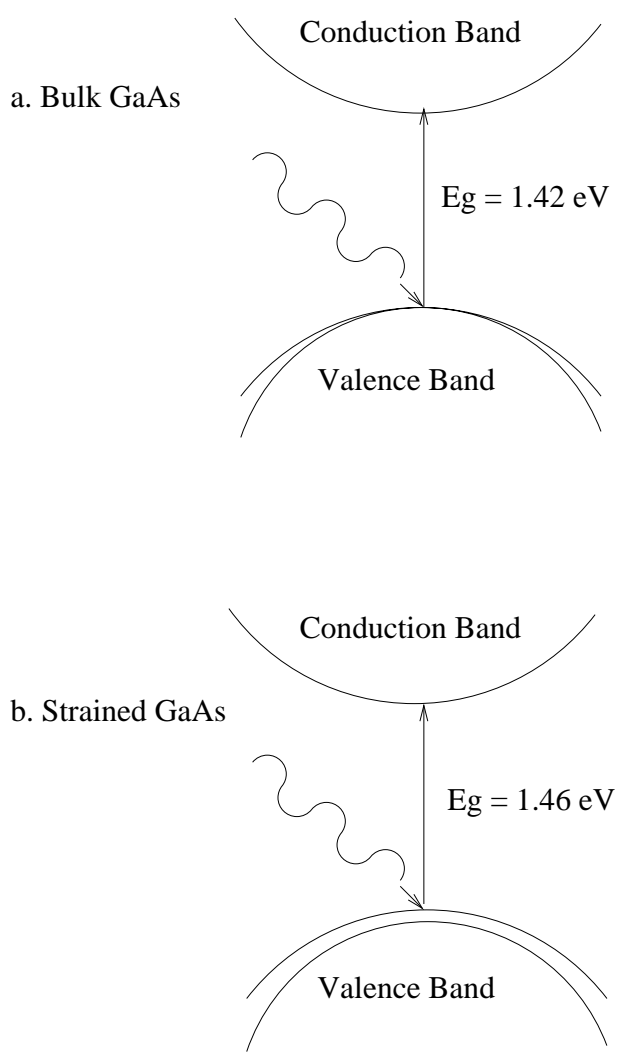
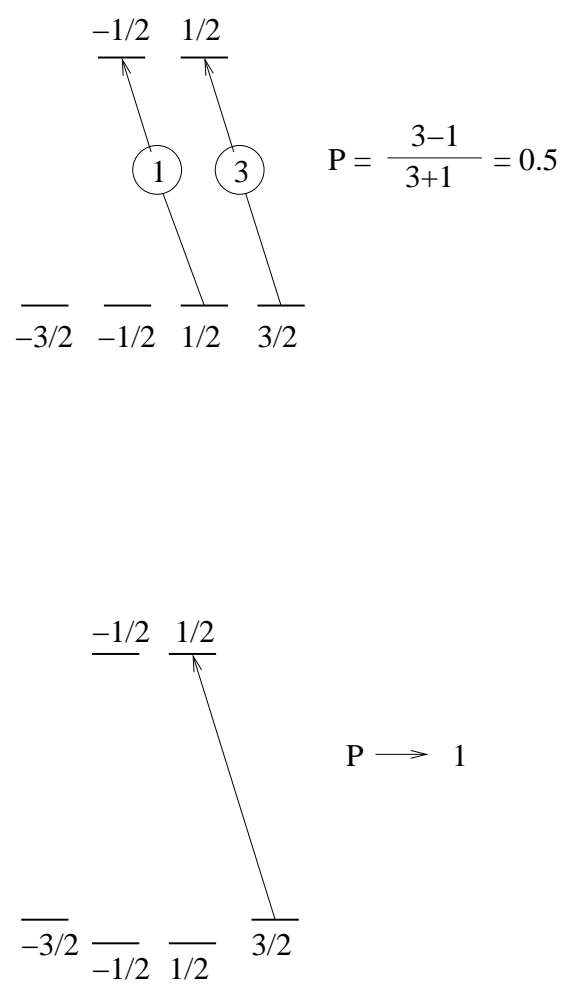

Figure 2.4: Energy differences between the valence and conduction bands for the bulk (a) and strained (b) GaAs crystals. The bulk GaAs shows a degenerate valance state, which limits the polarization to $50 \%$. Shown here are the left circularly polarized excitations. The circled numbers indicate the relative transition strengths.

$G^{0}$ target. Therefore, the spin must be rotated to be parallel or anti-parallel to the direction of motion of the beam when it hits the target, and not necessarily when it is being accelerated. The precession of the beam polarization occurs during transport due to the dipole magnetic fields, and is proportional to the beam's energy and bend angle

$$
\phi_{\text {spin }}=\frac{g-2}{2 m_{e}} \times E_{\text {beam }} \times \theta_{\text {bend }}
$$

where $g-2$ is the electron's anomalous magnetic moment, $m_{e}$ is the electron mass, $E_{b e a m}$ is the beam energy, and $\theta_{\text {bend }}$ is the bend angle. The rotation to compensate for this precession takes place in the injector region of the accelerator through the use of a Wien filter. A Wien filter consists of crossed electric and magnetic fields whose forces on the electron are equal and opposite, but produce a net rotation of the electron spin. Fig. 2.5 shows a simple schematic of what the wien 
filter does.
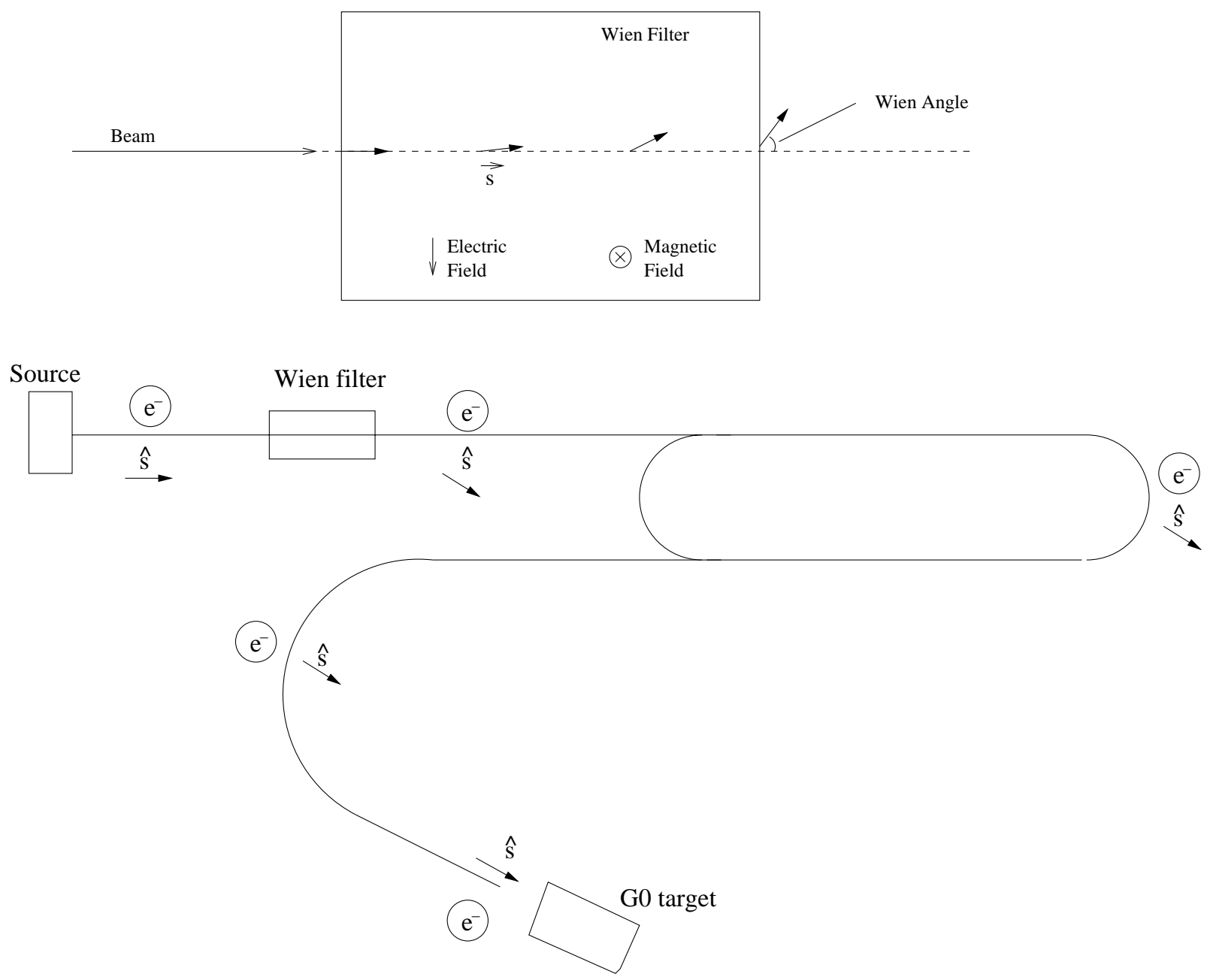

Figure 2.5: The effect of the wien filter.

The polarization of the beam is determined in Hall $\mathrm{C}$ by using the Møller polarimeter to measure the asymmetry in polarized $e^{-}-e^{-}$(Møller) scattering by detecting electrons scattered at $90^{\circ}$ center of mass when the beam strikes a thin magnetized iron target. This is possible due to the fact that this asymmetry is calculable using QED. The outer shell electrons in the iron atoms are polarized to be parallel or anti-parallel to the direction of motion of the beam by a $4 \mathrm{~T}$ solenoidal magnetic field. The asymmetry comes from the fact that the Møller scattering probability differs when the beam and target electron polarizations are aligned parallel or anti-parallel. The Møller asymmetry 
at a scattering angle of $90^{\circ}$ center of mass can be written as [64]

$$
A_{M}=-\frac{7}{9} P_{z}^{B} P_{z}^{T}+\frac{1}{9} P_{y}^{B} P_{y}^{T}-\frac{1}{9} P_{x}^{B} P_{x}^{T}
$$

where $P_{x}^{B}, P_{y}^{B}$ and $P_{z}^{B}$ are the beam polarizations along coordinates $x, y$, and $z$, and $P_{x}^{T}, P_{y}^{T}, P_{z}^{T}$ and are those of the target polarization. The target polarization is measured to be $8.04 \pm 0.02 \%$, contributing $0.2 \%$ to the overall Møller systematics. The Møller measurements are performed at a current of $2 \mu \mathrm{A}$, and thus needs to be extrapolated to the experimental condition of $40 \mu \mathrm{A}$. The summary of the overall beam polarization and its uncertainty contribution will be discussed in the analysis section.

\section{$G^{0}$ Beam Structure}

The polarized beam generated at the source is then accelerated to an energy of $3 \mathrm{GeV}$ before hitting the $G^{0}$ target. The superconducting radio-frequency $(\mathrm{RF})$ cavities used to accelerate the electrons operate at $1499 \mathrm{MHz}$, with alternating beam delivery to each hall at a rate of $\frac{1499}{3}=499 \mathrm{MHz}$. An $\mathrm{RF}$ separator is used to give the beam a transverse kick in order to steer it into one of the three halls or to recycle it back into the linacs for further acceleration. However, since the kick induced by the separator is relatively small, a Lambertson magnet, which is a three-channel septum magnet, is used to magnify the kick into one of the three halls. The simultaneous three hall delivery of beam is achieved using three different lasers (one for each hall) incident on a common GaAs cathode. The accelerator is designed such that it is able to deliver a maximum of $200 \mu \mathrm{A}$ of electron beam current at energies up to $6 \mathrm{GeV}$. The $G^{0}$ experiment ran with a $3 \mathrm{GeV}$ electron beam with an intensity of $40 \mu \mathrm{A}$.

The repetition rate of the $G^{0}$ beam is significantly different from what is usually delivered at JLab. With the conventional JLab beam structure of $499 \mathrm{MHz}$, each hall would receive a beam pulse every 2 ns. However, since the $G^{0}$ spectrometer identifies particles through tof separation, we require enough time separation between the pulses in order to detect the elastic protons. Therefore, the $G^{0}$ experiment requires a special beam structure with a pulse repetition rate of $31 \mathrm{MHz}$, or $32 \mathrm{~ns}$ between beam pulses. This gives adequate time to detect all particles, with the elastically scattered protons being detected between 18 and $25 \mathrm{~ns}$ after the beam pulse hits the target. 
The elastic proton yield must then be measured for both the + and - helicity states in order to determine the asymmetry. To this end, the helicity of the incoming beam is chosen to be flipped approximately every $\frac{1}{30}$ of a second, which we define to be a "macropulse" (MPS). Each MPS is exactly $\frac{1}{30}$ of a second (to cancel $30 \times \mathrm{n} \mathrm{Hz}$ noise), and the helicity Pockels cell takes $500 \mu$ s to flip the helicity. The helicity state of any given MPS is chosen such that every four MPS would form a "quartet" pattern of +-++ or -++- , with the first MPS of every quartet chosen pseudorandomly. The quartet structure is chosen such that the positive and negative helicity pulses are measured at the same average time, thereby eliminating the effect of linear drifts. The asymmetry is then calculated quartet-by-quartet

$$
\begin{aligned}
A_{q r t}^{a} & =\frac{Y_{1+}+Y_{4+}-Y_{2-}-Y_{3-}}{Y_{1+}+Y_{2-}+Y_{3-}+Y_{4+}} \\
A_{q r t}^{b} & =\frac{Y_{2+}+Y_{3+}-Y_{1-}-Y_{4-}}{Y_{1-}+Y_{2+}+Y_{3+}+Y_{4-}}
\end{aligned}
$$

where $Y$ is the yield, and the indices 1 through 4 represent the four MPS within a quartet, the indices $a$ and $b$ on the asymmetry represent the two types of quartets, and the $+(-)$ represent the positive(negative) helicity states. The final measured asymmetry is the mean of all quartet asymmetries measured over the entire run.

As a check of systematics, an insertable half-wave plate (IHWP) is located on the injector laser table. This is a half-wave plate that is remotely controlled through EPICS (see section 2.2) to be inserted/retracted from the laser path, and has the effect of flipping the handedness of the circular polarization and thus the beam helicity. This manual exchange of handedness is used to eliminate false asymmetries from the electronics. All data from the experiment are tagged as IHWP $=$ OUT or IN. When interchanging the IHWP state, the real physics asymmetry changes sign, but any false asymmetries from the electronics will not. Therefore, the sum of the asymmetries measured in the two states should be zero if there are no electronic false asymmetries. The voltage across the helicity Pockels cell, for example, will not change signs when the IHWP state is altered. If $A_{I N}+A_{O U T} \neq 0$, this suggests that the helicity signal is leaking from the Pockels cell into the experiment, resulting in false asymmetries.

The choice of the helicity pattern or the use of the IHWP and the luminosity monitors (discussed 
in the next section), however, will not eliminate all beam systematics. False asymmetries can arise from fluctuations in beam parameters that are correlated with the helicity. These helicitycorrelated (HC) beam parameter fluctuations induce changes in detector yield. If, for example, the beam position at the target changes when the helicity state is flipped, and the spectrometer is not symmetrically aligned around the target, this could cause a false asymmetry which can contaminate the measured physics asymmetry. Achieving the so-called parity quality of the beam with small helicity-correlated differences will impact several sources of false asymmetry, as will be discussed later in the analysis section. The next section will detail how various beam properties are measured and controlled.

\section{$2.2 \quad G^{0}$ Beam Properties}

\section{Luminosity Monitors}

In order to probe the effect of beam systematics, luminosity monitors are installed at small forward electron scattering angles. Four monitors are installed at an angle of $1.2^{\circ}$ and another four at 2.0 $0^{\circ}$ The azimuthal locations of the luminosity monitors can be seen in Fig. 2.6. The luminosity

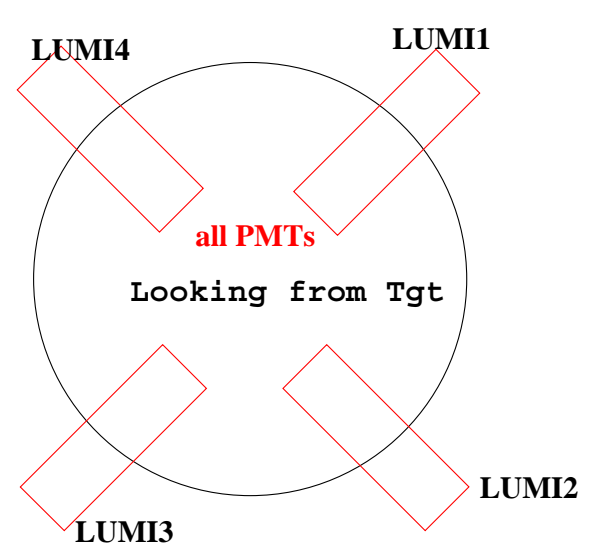

UPSTREAM

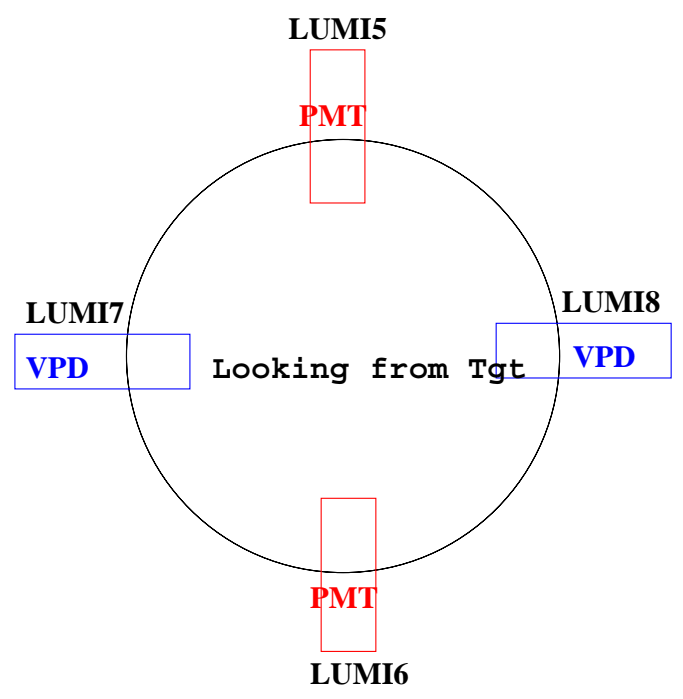

DOWNSTREAM

Figure 2.6: $G^{0}$ luminosity detector. The upstream detectors are in the even octants and are at a scattering angle of $2.0^{\circ}$. The downstream detectors are in the odd octants and are at a scattering angle of $1.2^{\circ}$ 
monitors are quartz detectors with dimensions of $3.5 \times 3.5 \times 7.6 \mathrm{~cm}$, all embedded in the beamline downstream of the magnet. Monitors 1 through 6 have PMTs to amplify the signal, whereas monitors 7 and 8 are vacuum photo-diodes (VPDs) which have low-noise pre-amps instead of PMTs.

These monitors are installed to detect electrons scattered into small forward angles. The electrons originate from elastic e-p scattering and polarized Møller (e-e) scattering off of orbital electrons, with both processes contributing about half of the total electron rate. One of the advantages that the luminosity monitors have over the FPDs (Focal Plane Detectors - see Section 2.5) is that the former have a considerably smaller asymmetry width. This is due to the fact that the counting rate is about $100 \times$ larger compared to the elastic proton rates seen by the FPDs, and hence the asymmetry width is about $10 \times$ smaller. Whereas the FPDs have a width of over $3000 \mathrm{ppm}$, the luminosity monitors show widths of about $300 \mathrm{ppm}$. This allows for a measurement of asymmetries with much higher precision. At small scattering angles, the asymmetry expected to be measured

by the luminosity monitor is $<0.1 \mathrm{ppm}$. With an asymmetry width of $300 \mathrm{ppm}$, the asymmetry can be measured with an uncertainty of about $0.1 \mathrm{ppm}$ over the entire forward angle production run; therefore, deviations from zero are due to false asymmetries. The luminosity monitors have another advantage that beam charge monitors (BCM) and beam position monitors (BPM) do not have, where they can detect electron scattering rates from small beam currents that BCMs and BPMs cannot see. This property of the luminosity monitors is useful in probing the effect of leakage beam from Halls A and B into Hall $\mathrm{C}$ as will be discussed in the analysis section.

\section{Parity Quality Beam}

The beam parameters monitored for helicity-correlation involve beam charge, $x$ and $y$ beam posi- 
tion, $x$ and $y$ beam angle, and beam energy

$$
\begin{aligned}
& \text { Charge asymmetry }=A_{q}=\frac{I_{1}+I_{4}-I_{2}-I_{3}}{I_{1}+I_{2}+I_{3}+I_{4}} \\
& \text { x position differences }=\delta_{x}=\frac{x_{1}+x_{4}-x_{2}-x_{3}}{2} \\
& \text { y position differences }=\delta_{y}=\frac{y_{1}+y_{4}-y_{2}-y_{3}}{2} \\
& \text { x angle differences }=\delta \theta_{x}=\frac{\theta x_{1}+\theta x_{4}-\theta x_{2}-\theta x_{3}}{2} \\
& \text { y angle differences }=\delta \theta_{y}=\frac{\theta y_{1}+\theta y_{4}-\theta y_{2}-\theta y_{3}}{2} \\
& \text { Energy differences }=\delta E=\frac{E_{1}+E_{4}-E_{2}-E_{3}}{2}
\end{aligned}
$$

where the indices 1 through 4 indicate the four MPS within a quartet. These definitions of the helicity-correlated beam parameter differences are for the IHWP IN state. For the IHWP OUT state, the above definitions need to be multiplied by -1 .

The false asymmetry induced by HC differences is

$$
A_{\text {false }}=\frac{1}{2 Y} \Sigma_{i} \frac{d Y}{d P_{i}} \delta_{P i}
$$

where the slope $\frac{d Y}{d P_{i}}$ represents the response of the measured detector yield to changes in the six beam parameters, and the $\delta_{P i}$ 's are the six HC beam parameters.

The beam parameters are measured using a set of beam charge monitors (BCMs) (RF cavities) and stripline beam position monitors (BPMs) [62] in Hall C. The $\mathrm{x}$ and $\mathrm{y}$ positions and $\mathrm{x}$ and $\mathrm{y}$ angles at the target are determined using two such BPMs (IPM3HG0 and IPM3HG0B) a few meters upstream of the target. Each BPM is capable of measuring both the $\mathrm{x}$ and $\mathrm{y}$ position of the beam, so the two transverse positions and two angles of the beam at the target can be extrapolated from those measurements. There are no optical elements between these two BPMs or between the BPMs and the target that can focus or steer the beam. The energy of the beam is measured by a BPM (IPM3HC12) located near the entrance of the hall in a dispersive section of the beamline. The sensitivities of the various HC beam parameters can be found in Table 2.1.

Two methods are used to measure the slopes in Eqn. 2.14. One is to simply use the natural 


\begin{tabular}{|c|c|}
\hline Beam parameter & Resolution \\
\hline Charge & $1000 \mathrm{ppm}$ \\
\hline Beam Position & $30 \mu \mathrm{m}$ \\
\hline Beam Angle & $3 \mu \mathrm{m}$ \\
\hline Beam Energy & $20 \mathrm{keV}$ \\
\hline
\end{tabular}

Table 2.1: Resolutions of HC beam parameters seen in the experiment. The resolution does not come solely from the BCMs and BPMs, but is also dependent on the tune of the beam itself. Therefore, these resolution varied in time. Shown above are typical values seen over the production run.

beam motion (NBM) to see how much the rates in the detectors change as a function of the beam parameters. However, during the first engineering run, the slopes measured through NBM were seen to be unstable over time, although this problem was not observed during the production run, presumably due to better tuning of the beam. For this reason, a set of steering coils are used upstream of the target to artificially dither the beam by larger amounts in order to increase the dynamic range of the beam parameters. These runs are designated to be coil pulsing (CP) runs. The slopes and their false asymmetries will be discussed later in the analysis section.

The other contribution to the false asymmetry in Eqn. 2.14 is the helicity-correlation (HC) seen in the beam parameters. There can be several mechanisms that can cause these helicity-correlations. However, all are tied in some way to the helicity Pockels cell since this is the optical element which determines the polarization state of the electron beam. One way in which the HPC can induce HC beam parameter fluctuations is simply through steering. Since all crystalline materials exhibiting a Pockels effect are also piezo-electric, the application of a voltage across such a crystal can cause its shape to distort. Such distortions can cause the laser to refract off of the desired beam axis by different amounts for each of the two polarization states. If the cell causes the laser beam passing through it to be steered differently between the two helicity states, the laser will hit the cathode in different locations between the two states, and the electron beam will have two different initial positions, causing HC position differences. Furthermore, if the cathode itself has a significant $Q E$ gradient across its surface, it can photo-emit different amounts of charge depending on where the laser hits. This mechanism will convert HC steering into helicity-correlated charge asymmetry.

Another source of charge asymmetry comes from the fact that the HPC does not create a perfectly circularly polarized light, and that the cathode itself has some analyzing power. With a 
$99.98 \%$ circularly polarized light, the residual linear polarization is about $1.4 \%$. Since this residual linear polarization will rotate when the handedness of the circular polarization is flipped, the analyzing power of the cathode can cause a helicity-correlated photo-emission difference. The analyzing power of the strained GaAs crystal is measured in the injector by measuring the beam current as the rotating half-wave plate (RHWP) is rotated. The RHWP, located on the injector laser table, is a half-waveplate mounted on a stand which can rotate the waveplate about the laser beam axis. Rotating the RHWP has no effect on the circular polarization but rotates any residual linear polarization. The analyzing power is measured while the helicity Pockels cell is turned off such that the laser hitting the cathode is linearly polarized. The intensity of the beam would then

modulate as $\cos ^{2}(\theta)$ (Malus' Law), where $\theta$ is the angle between the linear polarization and the analyzing power of the cathode. The measurement is done in the injector using the Polarized Cup (PCUP) which is a Faraday cup (measuring total charge by absorption). The measurement can be seen in Fig. 2.7. The analyzing power is defined as the amplitude of the sine oscillation, and is measured to be $11 \%$.

\section{Beam Transport and Adiabatic Damping}

Although the helicity-correlation in the beam has its origins at the helicity Pockels cell, how this correlation manifests itself at the $G^{0}$ target is further complicated by the transport of the beam, especially the transport through the injector.

Toward the beginning of the run, it was thought that HC position differences would be suppressed by "adiabatic damping" of beam motion. Adiabatic damping describes the reduction in transverse motion of the beam as it is accelerated. It originates in the relative reduction of the transverse momentum components compared to the longitudinal momentum (the longitudinal momentum is increased by the acceleration). The equation of motion governing this property is

$$
x^{\prime \prime}(s)+\left(\frac{p^{\prime}}{p}\right) x^{\prime}(s)+K(s) x(s)=0
$$

where $x$ is the transverse position of the beam, $s$ is the distance along the accelerator, and $K(s)$ 


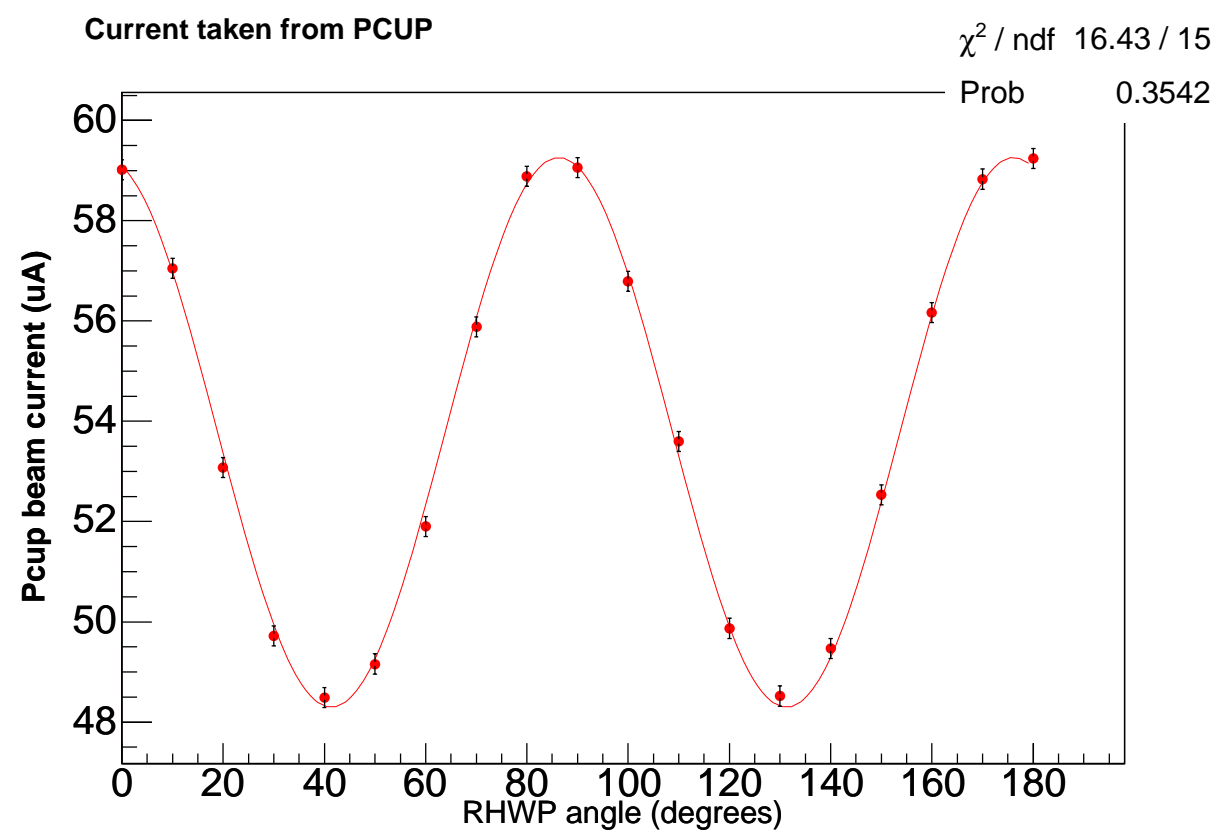

Figure 2.7: Plot showing the analyzing power of the cathode. The analyzing power is calculated as the amplitude of the sine oscillation.

reflects the transverse focusing primarily due to quadrupole fields. The solution to this equation is

$$
x(s)=\sqrt{\epsilon} \sqrt{\beta(s)} \sqrt{\frac{p_{0}}{p}} \cos (\Psi(s)+\phi)
$$

where $\epsilon$ is the transverse emittance of the beam, $\beta(s)$ is the beta function, $\Psi(s)$ is the betatron phase advance (both governed by the optical layout of the beamline), and $\phi$ is the launch phase. The transverse fluctuation of the beam position should decrease as $\sqrt{\frac{p_{0}}{p}}$. The kinetic energy of the electron beam at injection is $100 \mathrm{keV}$, with a final hall energy of $3 \mathrm{GeV}$. Therefore, the total damping from injection to hall should be $\sqrt{\frac{335 \mathrm{keV}}{3 \mathrm{GeV}}}=0.01057$, or a suppression factor of about 95 . However, tests during the fall-winter of 2003 showed that the damping factor was significantly less than this theoretical value. Fig. 2.8 shows the damping factor seen for different sections of the injector.

The maximum damping seen from these tests is $<30$. In particular, there is little adiabatic damping seen in the injector (where the damping factor should be $\sqrt{\frac{335 \mathrm{keV}}{60 M e V}}=0.0747$, or a sup- 

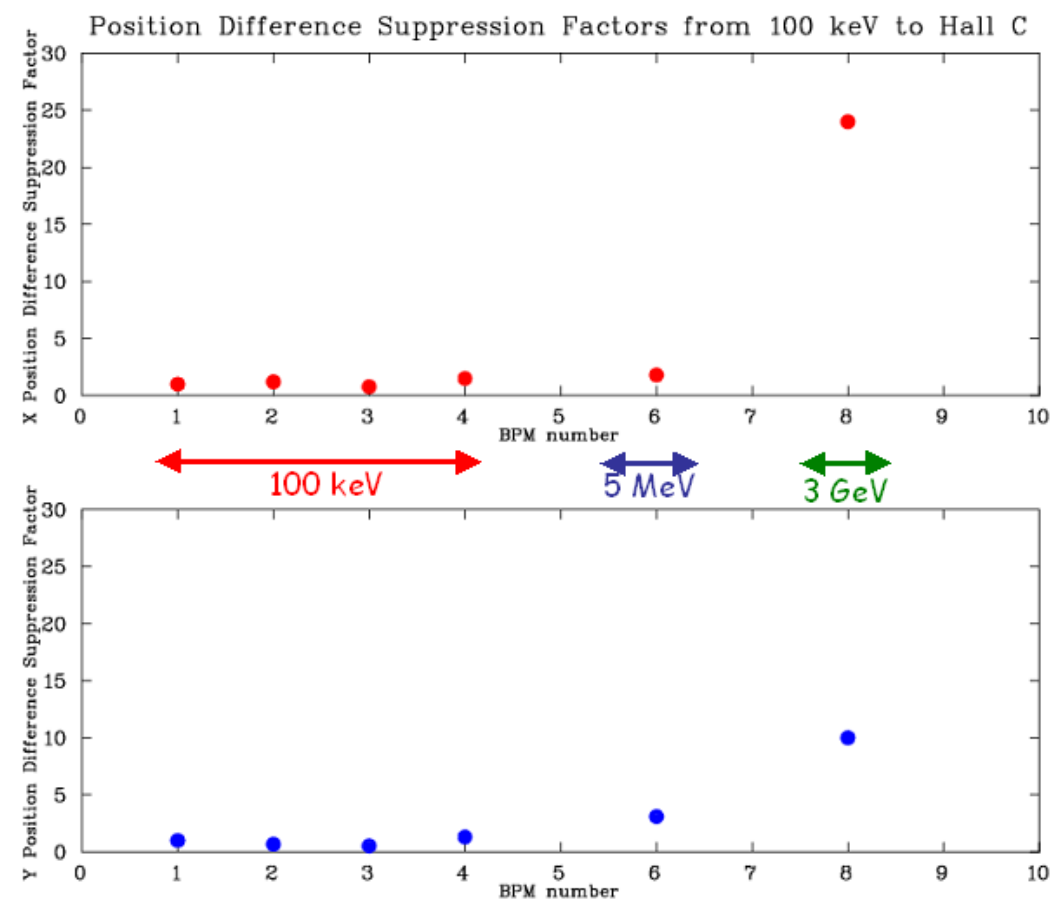

Figure 2.8: Adiabatic damping factor seen for different locations along the accelerator and in Hall C. Each point represents the ratio of position differences measured at a particular BPM and the first BPM in the injector (BPM0L02). The $100 \mathrm{keV}$ region is the front end of the injector, and 3 $\mathrm{GeV}$ corresponds to measurements made in Hall $\mathrm{C}$ near the $G^{0}$ target.

pression factor of about 13), which is contributing significantly to the overall lack of damping.

One possible reason for the lack of adiabatic damping is the fact that the beam is scraping in the injector due to the unique $G^{0}$ beam structure [63]. A property of the $31 \mathrm{MHz} G^{0}$ beam structure that is different from the conventional $499 \mathrm{MHz}$ beam is that in order to achieve a high beam current of $40 \mu \mathrm{A}$ with $\frac{31 \mathrm{MHz}}{499 \mathrm{MHz}}=\frac{1}{16}$ the pulse frequency, more electrons need to be squeezed into each beam bunch. The bunch charge of the $G^{0}$ beam is $1.6 \mathrm{pC}$, which is an order of magnitude larger than what is usually delivered at JLab. This creates problems in terms of transport loss of the electron beam through the $10 \mathrm{~m}$ long, $100 \mathrm{keV}$ section of the injector. A schematic of the injector from the $100 \mathrm{keV}$ region to the $45 \mathrm{MeV}$ region can be seen in Fig. 2.9. Transmission loss appears when the Coulomb repulsion from the high bunch charge results in the transverse and longitudinal lengthening of the beam bunch. Typically, transmission loss occurs at two circular apertures (called A1 and A2) and the chopper slit. The A1 and A2 apertures are 4 and $6 \mathrm{~mm}$ diameter holes which limit the transverse emittance of the beam before it is accelerated through the RF cavities. The 


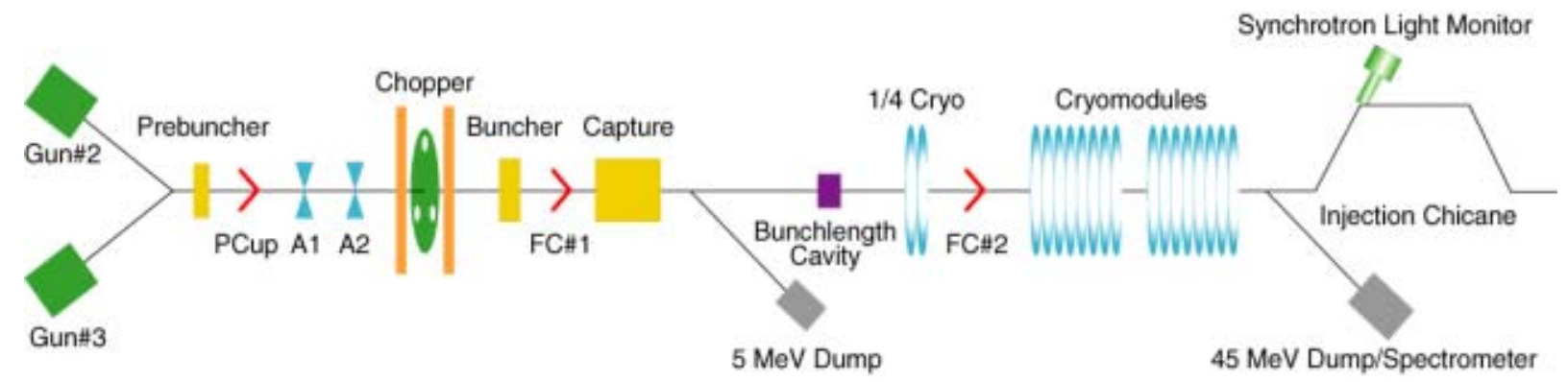

Figure 2.9: Injector schematic.

chopper system is used to assure initial timing and longitudinal structure of the beams for all three halls. It is a rotating disc with three apertures, each of which allows the passage of one of the three hall beams. The system operates at $499 \mathrm{MHz}$, and is timed such that it defines a phase acceptance from 0 to $110 \mathrm{ps}$ for each beam. Any beam outside this window would not be synchronized to the RF cavity frequency, and thus will not go through the bunching and acceleration process properly. Therefore any such beam is stopped by the choppers. Thus, while A1 and A2 are spatial apertures that limit the transverse emittance, the chopper can be thought of as a temporal aperture which limits the longitudinal emittance of the beam. In order to compensate for the bunch lengthening due to high bunch charge, a pre-buncher cavity is placed before the chopper to rebunch the beam so that its length at the chopper remains within the longitudinal acceptance. Further adjustments such as changing the focusing and laser spot size to reduce initial bunch charge density are done to mitigate the Coulomb repulsion after the chopper. The beam is then bunched again before it is sent through the acceleration process.

With the usual $499 \mathrm{MHz}$ beam structure, the transmission is typically 100\% through these beam elements. However, with the large bunch charge beam structure, the transmission for the forward angle $G^{0}$ beam was about $75 \%$ throughout the run. This transmission loss due to scraping is thought to be one of the reasons for the poor adiabatic damping with scraped particles being transported at the edge of the acceptance of the accelerator. Significant effort was made in part by both the $G^{0}$ experiment and by the JLab accelerator group to understand the lack of adiabatic damping in the injector, but a solution has not yet been found.

Another potential source of the lack of adiabatic damping comes from betatron mismatch. The 
transport from any point within the accelerator to another downstream point in the JLab injector and accelerator can be described by a $6 \times 6$ linear transport matrix

$$
\left(\begin{array}{c}
x_{f} \\
x_{f}^{\prime} \\
y_{f} \\
y_{f}^{\prime} \\
l_{f} \\
\delta_{f}
\end{array}\right)=\left(\begin{array}{llllll}
m_{11} & m_{12} & m_{13} & m_{14} & m_{15} & m_{16} \\
m_{21} & m_{22} & m_{23} & m_{24} & m_{25} & m_{26} \\
m_{31} & m_{32} & m_{33} & m_{34} & m_{35} & m_{36} \\
m_{41} & m_{42} & m_{43} & m_{44} & m_{45} & m_{46} \\
m_{51} & m_{52} & m_{53} & m_{54} & m_{55} & m_{56} \\
m_{61} & m_{62} & m_{63} & m_{64} & m_{65} & m_{66}
\end{array}\right)\left(\begin{array}{c}
x_{i} \\
x_{i}^{\prime} \\
y_{i} \\
y_{i}^{\prime} \\
l_{i} \\
\delta_{i}
\end{array}\right)
$$

where $x_{i}, x_{i}^{\prime}, y_{i}$, and $y_{i}^{\prime}$ are the transverse positions and angles, $l_{i}$ is the phase difference between the two longitudinal points, $\delta$ is the momentum spread, and the subscripts $i$ and $f$ refer to the initial and final longitudinal states of the beam. Although the beam can be represented by the above $6 \times 6$ matrix in the most general case, if one assumes decoupled motion, it can be represented by a set of courant-snyder (or twiss) parameters. These twiss parameters can be used to describe the decoupled motion in the form of a phasespace ellipse as seen in Fig. 2.10. Ideally, the phasespace would

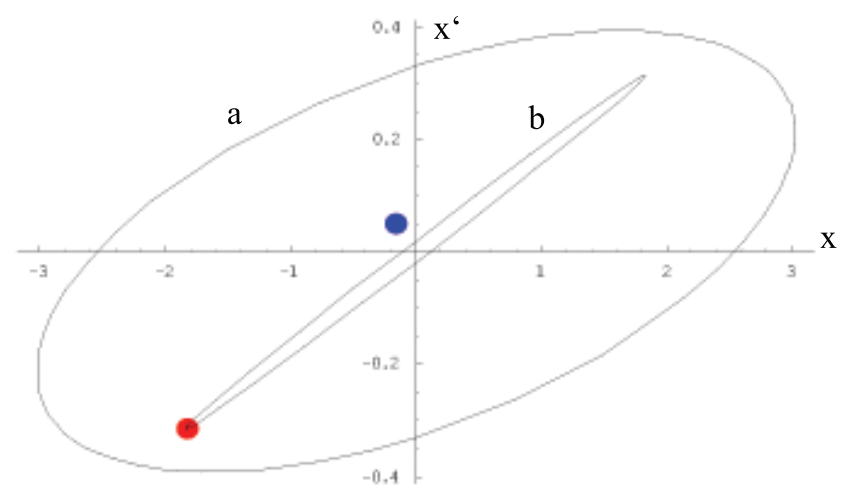

Figure 2.10: Phasespace ellipse. This ellipse is taken from a study of the beam orbit using a PZT mirror (common PZT instead of the Hall C PZT). In (a), the orbit is reasonable, while (b) clearly shows a large correlation between the transverse position and angle.

appear circular (similar to orbit (a) in Fig. 2.10). However, certain conditions such as mismatches in the beam optics from deviations in magnetic elements from design, or unaccounted for focusing from RF couplers can skew the phase-space (as in orbit (b)). This skewness, or "stretching" of the ellipse, may result in orbit "blow-up", which can counter the effect of damping. The examination 
of this orbit blow-up continues to be studied at JLab.

\section{Beam Feedback}

In order to control the HC beam parameters, a beam feedback mechanism is implemented as seen in Fig. 2.12. The correction is done at the source by a set of three optical elements; the RHWP, the IA (intensity asymmetry) cell, and the PZT (piezo-electric transducer) mirror. The RHWP aligns the residual linear polarization of the two helicity states to be at equal angles, to the extent possible, with respect to the axis of the analyzing power of the crystal. Thus, both helicity states will have equal photo-emission, again from $I \cos ^{2}(\theta)$, nulling the charge asymmetry coming from the residual linear polarization component.

The IA cell is located on the injector laser table (Fig. 2.3), and is used as a charge feedback system to null any remaining charge asymmetry seen in the beam. The IA changes the laser intensity (incident on the photo-cathode) by acting as a variable neutral density filter, and thus changing the total charge emitted from the cathode. The feed back device uses the IA and a BCM in Hall $\mathrm{C}$ to feedback on the beam charge by modulating the laser intensity during helicity-reversal. The elements (starting from the most upstream element) are: upstream linear polarizer, waveplate $\left(\frac{\lambda}{10}\right)$, Pockels cell, and a downstream linear polarizer. The light incident on the Pockels cell has an elliptical polarization due to the waveplate. This is preferred over linearly polarized light, since the elliptical polarization would assure that a fraction of the light is not aligned along either the fast or slow axis (which would induce no phase retardation). Fig. 2.11 shows how the laser's intensity is modulated by manipulating its polarization state.

Voltage is applied to the Pockels cell at $30 \mathrm{~Hz}$, with zero in one helicity state and $\mathrm{V}$ in the other helicity state. By doing this, the Pockels cell can induce a different relative phase on the light depending on the helicity state. The DSLP (downstream linear polarizer) then acts as an analyzer to allow light of different intensities to pass through depending on the phase of the incident light. The above IA cell can thus be used to correct the beam charge in a helicity-dependent manner.

The PZT mirror is driven with a Thorlabs KC1-PZ piezo-electric transducer [56], and used on the injector laser table as a position feedback system to null any position differences present in the beam. It is used to modulate the position of the laser on the cathode at helicity-reversal by 


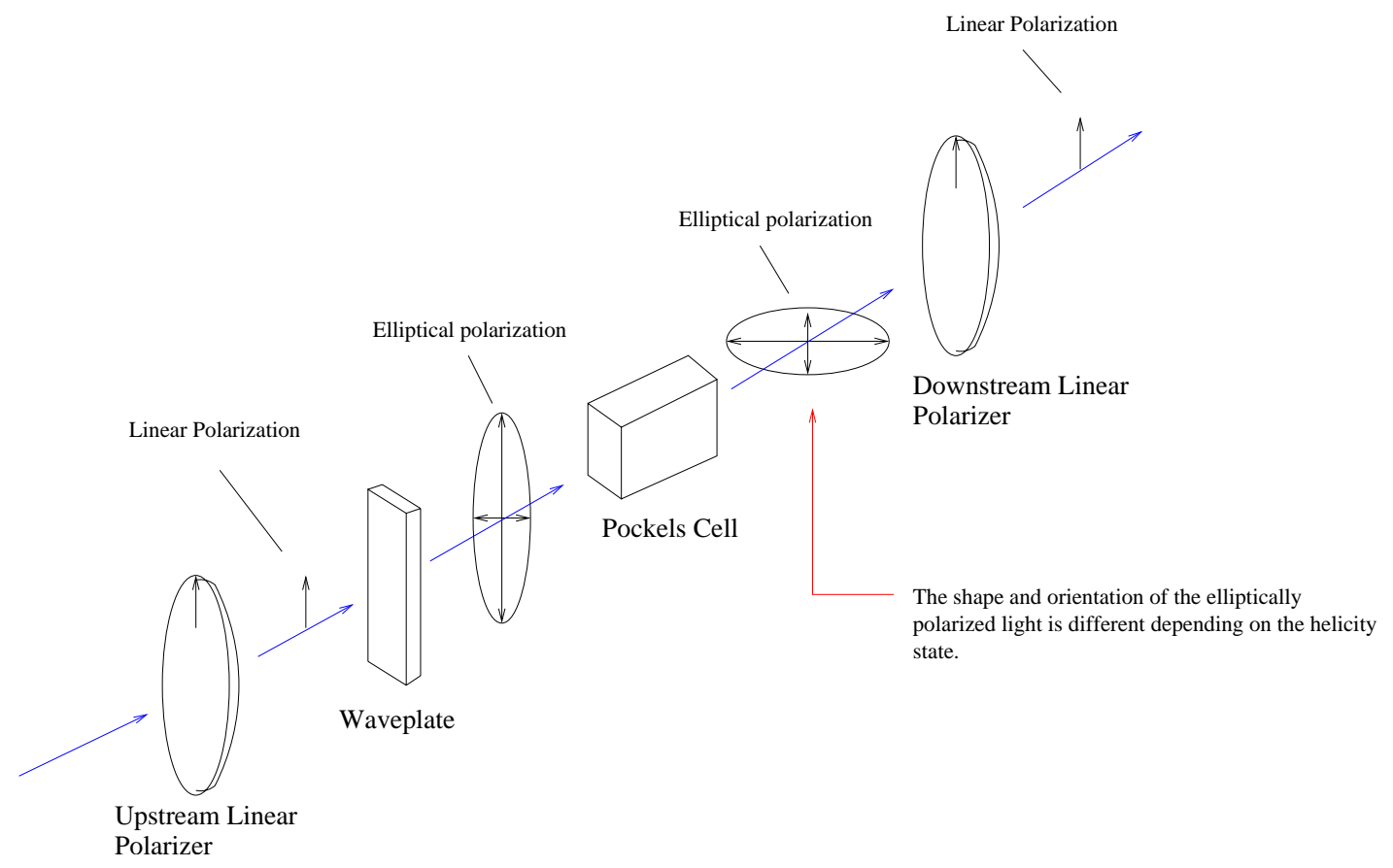

Figure 2.11: Laser intensity modulation through the use of an IA cell. The waveplate induces a slight elliptical polarization to shift the laser polarization off the pockels cell's fast and slow axes. If the waveplate is not present, and the linearly polarized light is incident on the pockels cell exactly aligned to one of these axes, then applying the voltage across the cell would not induce a relative phase retardation between the two helicity states.

applying different voltages for each helicity state.

In order to use the IA and the PZT properly as a feedback device, one needs to know how much charge asymmetry and position difference can be induced as a function of the voltage applied to each of these devices. The slopes of the charge asymmetry and position differences with respect to the IA and PZT voltages are determined by making measurements of $A_{q}$ and $\delta_{x(y)}$ at the two extreme voltage values as well as at their median values. We call these calibrations the IA scan and the PZT scan. The voltage range applicable across the IA is 0 to $150 \mathrm{~V}$, and across the PZT is -12 to $12 \mathrm{~V}$ along both $\mathrm{x}$ and $\mathrm{y}$. Therefore, an IA scan involves taking measurements at 0,75 , and 150 $\mathrm{V}$ (which correspond to ADC inputs of 0,5 , and $9 \mathrm{~V}$ ), while a PZT scan is a set of measurements at $-12,0$, and $12 \mathrm{~V}$ (again, corresponding to ADC inputs of 0,5 , and $9 \mathrm{~V}$ ). Setting the RHWP to null the charge asymmetry is the last step prior to engaging the feedback. Since the RHWP can rotate $360^{\circ}$ around the laser path, it is calibrated by measuring the charge asymmetry at different values of the RHWP angle. We call this an RHWP scan. The RHWP is then placed at an angle 
which shows a zero-crossing in charge asymmetry.

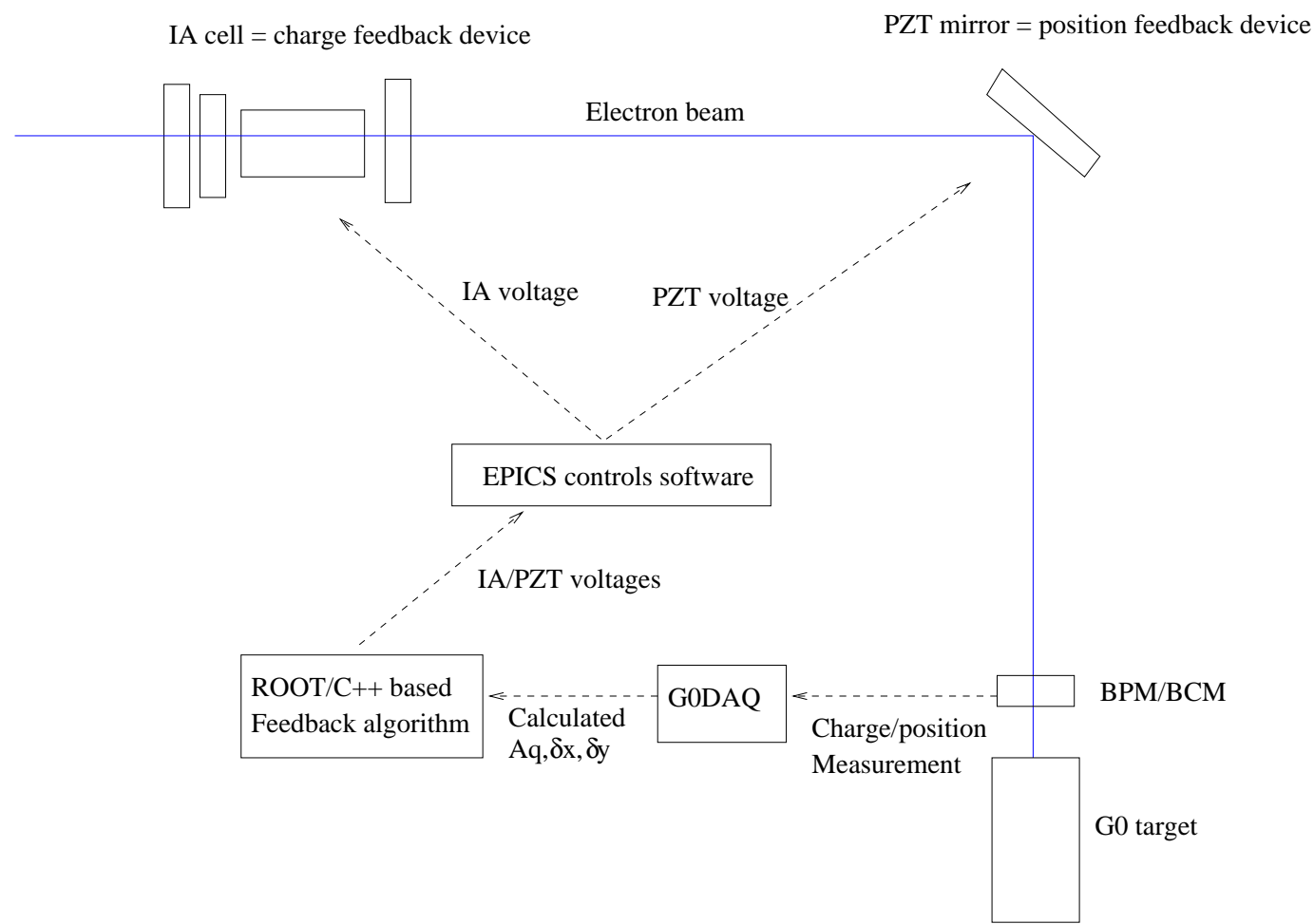

Figure 2.12: Schematic of the beam feedback system. HC charge asymmetry and position differences are measured near the target, and are sent back to the IA and PZT mirrors at the source to null out the helicity-correlation.

There are two main control systems used for the feedback. One is primarily used to control the accelerator and source hardware, while another is used primarily by $G^{0}$. The various optical elements in the injector as well as the accelerator are controlled through the Experimental Physics and Industrial Control System (EPICS) and its GUI extension called the Motif Editor and Display Manager (MEDM). The $G^{0}$ part of the feedback system is controlled through ROOT/C++. The HC beam parameters are measured, and are processed through a ROOT-based analysis code to determine what the proper IA/PZT voltages are to feed back. These voltages are then sent through the accelerator EPICS system to the IA and PZT devices. The basic flow of the feedback scheme can be seen in Fig. 2.12.

The feedback algorithm must be described with a $3 \times 3$ matrix because the effects of the IA and PZT mirror turn out to be coupled. The voltages required for the IA and PZT to null out a certain 
amount of charge asymmetry and position difference can be calculated from

$$
\left(\begin{array}{c}
A_{q, \text { meas }} \\
\delta_{x, \text { meas }} \\
\delta_{y, \text { meas }}
\end{array}\right)=-\left(\begin{array}{ccc}
\frac{d A_{q}}{d I A} & \frac{d \delta_{x}}{d I A} & \frac{d \delta_{y}}{d I A} \\
\frac{d A_{q}}{d P Z T_{x}} & \frac{\delta_{x}}{d P Z T_{x}} & \frac{\delta_{y}}{d P Z T_{x}} \\
\frac{d A_{q}}{d P Z T_{y}} & \frac{\delta_{x}}{d P Z T_{y}} & \frac{\delta_{y}}{d P Z T_{y}}
\end{array}\right)\left(\begin{array}{c}
I A_{\text {corrected }} \\
P Z T_{x, \text { corrected }} \\
P Z T_{y, \text { corrected }}
\end{array}\right)
$$

where the $3 \times 3$ matrix shows the slopes determined from the IA and PZT scans, $A_{\text {qmeas }}, \delta_{x m e a s}$, and $\delta_{y m e a s}$ are the measured charge asymmetry and position differences that must be nulled, and $V_{I A}$ and $V_{P Z T X(Y)}$ are the voltages that should be applied across the IA and PZT devices in order to null out the helicity-correlation. In the course of testing the IA/PZT feedback system during the first engineering run, it was observed that the slopes shown in Eqn. 2.18 tend to change over time. This change was seen on timescales of hours to days. In order to probe the source of this instability, an independent study of these two devices was done on a test laser table. A description and the test and the conclusion drawn from it can be found in Appendix A.1. The test showed that the time-variation of the IA and PZT responses did not originate from the devices themselves, which indicate that the problem existed in the transport of the electron beam from the cathode to the hall.

The $\mathrm{x}$ and $\mathrm{y}$ position differences of the beam are primarily controlled by the $2 \mathrm{x} 2 \mathrm{PZT}$-position difference submatrix, $\left(\begin{array}{cc}\frac{d P Z T_{x}}{\delta_{x}} & \frac{d P Z T_{x}}{\delta_{y}} \\ \frac{d P Z T_{y}}{\delta_{x}} & \frac{d P Z T_{y}}{\delta_{y}}\end{array}\right)$. However, in order for the position feedback to be effective, the PZT motions of the mirror in the $\mathrm{x}$ and $\mathrm{y}$ directions must generate motions of the beam at the target, $\delta_{x}$ and $\delta_{y}$, that are large enough to correct the possible errors. If, for example, the dominant motions of the beam from the $\mathrm{x}$ and $\mathrm{y}$ motion of the mirror are in the $\mathrm{y}$ direction (because of coupling of the $\mathrm{x}$ and $\mathrm{y}$ directions in the accelerator, whose magnitude unfortunately changes in time), then the PZT mirror would not be able to correct for $\mathrm{x}$ position differences. This characteristic of the feedback system is abbreviated as the "beam orthogonality". Measurements show breakdown of orthogonality as the beam travels down the injector. However, although orthogonality is broken in the hall, there is usually (see below) sufficient dynamic range in $\delta_{x}$ and $\delta_{y}$ to null out the helicity-correlation in charge and position. The measurement of this orthogonality is summarized in Appendix A.2. 
There were instances where the dynamic range of the PZT was not sufficient to feedback on the position differences. In such cases, quadrupole magnets in the Hall are adjusted to reduce the position differences and increase the dynamic range of the PZT. The quadrupole magnets also have the effect of reducing the widths seen in the position differences. The position differences are accumulated over a finite amount of time before they are sent as inputs to the feedback algorithm, such that the values are known with a minimum amount of statistics. When the noise associated with the position differences become too large to effectively feedback on a reasonable timescale, the quadrupoles can be used to improve the statistical widths. The effect of adjusting the quadrupole fields can be seen in Appendix A.3.

An additional complication that arose when operating the feedback was a correlation between $A_{q}$ and $\delta_{x(y)}$ that is independent of either the IA or the PZT, presumably caused by scraping of the electron beam at apertures in the accelerator - although this was not proven. This correlation was seen during calibrations of both the IA and RHWP. Since the IA and the RHWP modulate the beam intensity through completely independent mechanisms, the fact that the correlation exists in both scans indicate that the source of this correlation is independent of both devices. Since the feedback matrix in Eqn. 2.18 assumes no direct correlation between $A_{q}$ and $\delta_{x(y)}$ other than what is induced by the IA or the PZT, this additional correlation can cause the feedback system to malfunction. However, a solution to this problem was found and is outlined in Appendix A.4. The solution regresses the effect of this additional correlation out of the feedback matrix by directly measuring this correlation using the RHWP. An example of the overall performance of the feedback system can be seen in Fig. 2.13.

The frequency of feedback is chosen such that enough statistics can be accumulated to know the central values of $A_{q}$ and $\delta_{x(y)}$ to within 10's of ppm and 100's of nm, and to assure that the average $\mathrm{HC}$ differences can be nulled to within the specifications in a reasonable amount of time (hours, instead of days). The IA fed back on the charge asymmetry every 5 minutes, while the PZT fed back every 30 minutes. The feedback of the two systems are synchronized such that every 30 minutes, both the IA and the PZT would do a full 3x3 feedback.

The HC beam parameters can be seen in Fig. 2.14 plotted against run number. The specifications for parity quality beam outlined for the $G^{0}$ forward angle measurement, as well as the final 

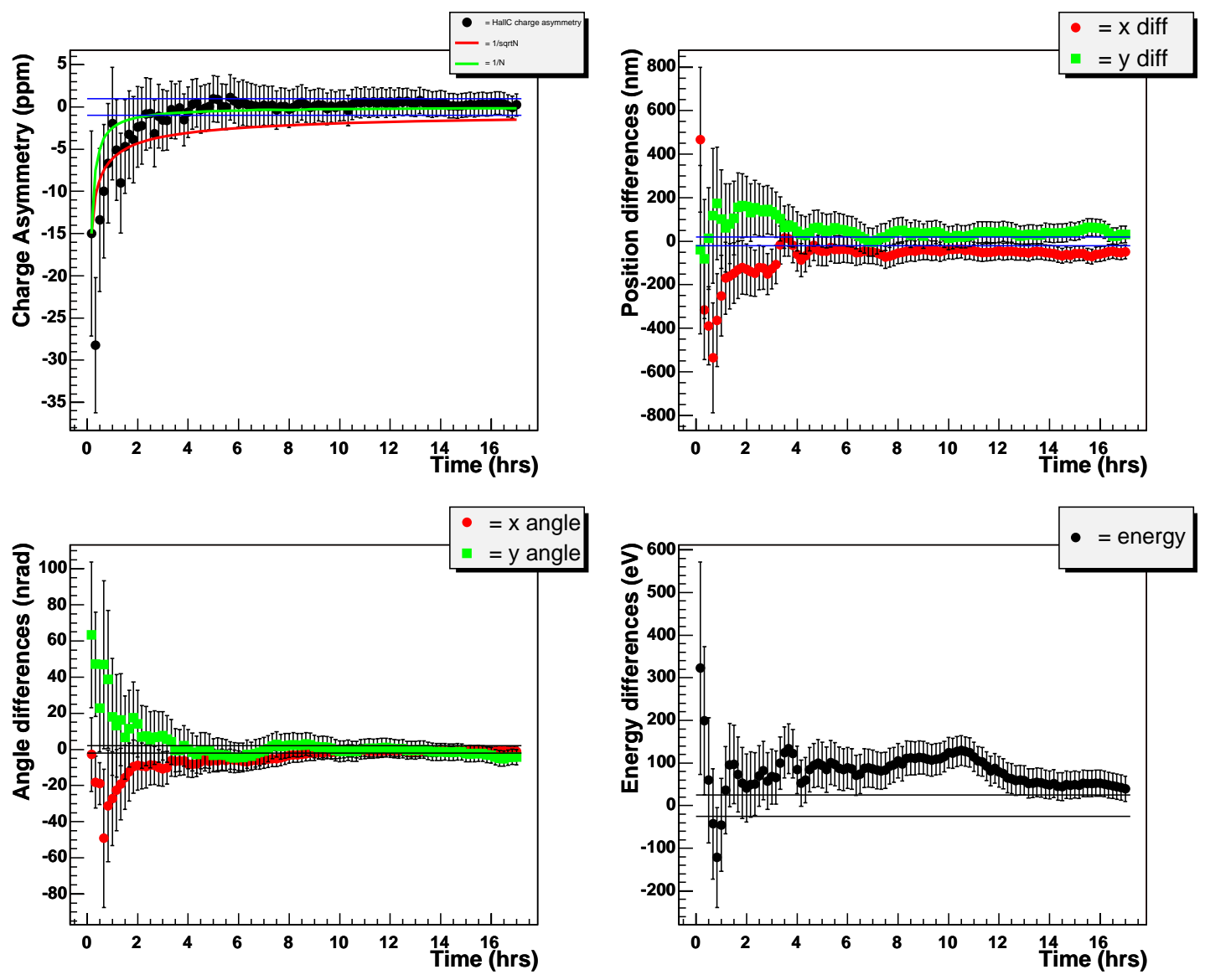

Figure 2.13: HC differences integrated over several hours. Each point represents 5 minutes worth of quartets. The green line shows the theoretical convergence rate if the feedback worked perfectly. The red line shows the $\frac{1}{\sqrt{N}}$ convergence to see if the feedback is beating the statistics. The blue horizontal lines indicate the specifications to within which the HC parameters need to be reduced.

achieved values can be found in Table 2.2. The achieved values are the averages over all good runs defined later in the analysis section. Without the feedback, the HC differences are seen to be of

\begin{tabular}{|c|c|c|}
\hline HC parameter & Achieved $\left(\frac{I N-O U T}{2}\right)$ & Specifications \\
\hline Charge asymmetry $(\mathrm{ppm})$ & $-0.14 \pm 0.32$ & 1 \\
\hline x position difference $(\mathrm{nm})$ & $3 \pm 4$ & 20 \\
\hline y position difference $(\mathrm{nm})$ & $4 \pm 4$ & 20 \\
\hline x angle difference $(\mathrm{nrad})$ & $1 \pm 1$ & 2 \\
\hline y angle difference $(\mathrm{nrad})$ & $1.5 \pm 1$ & 2 \\
\hline Energy difference $(\mathrm{eV})$ & $29 \pm 4$ & 75 \\
\hline
\end{tabular}

Table 2.2: Helicity-correlated beam parameters achieved during the $G^{0}$ forward angle production run. The middle column shows the achieved values, while the right-most column shows the specifications to within which 

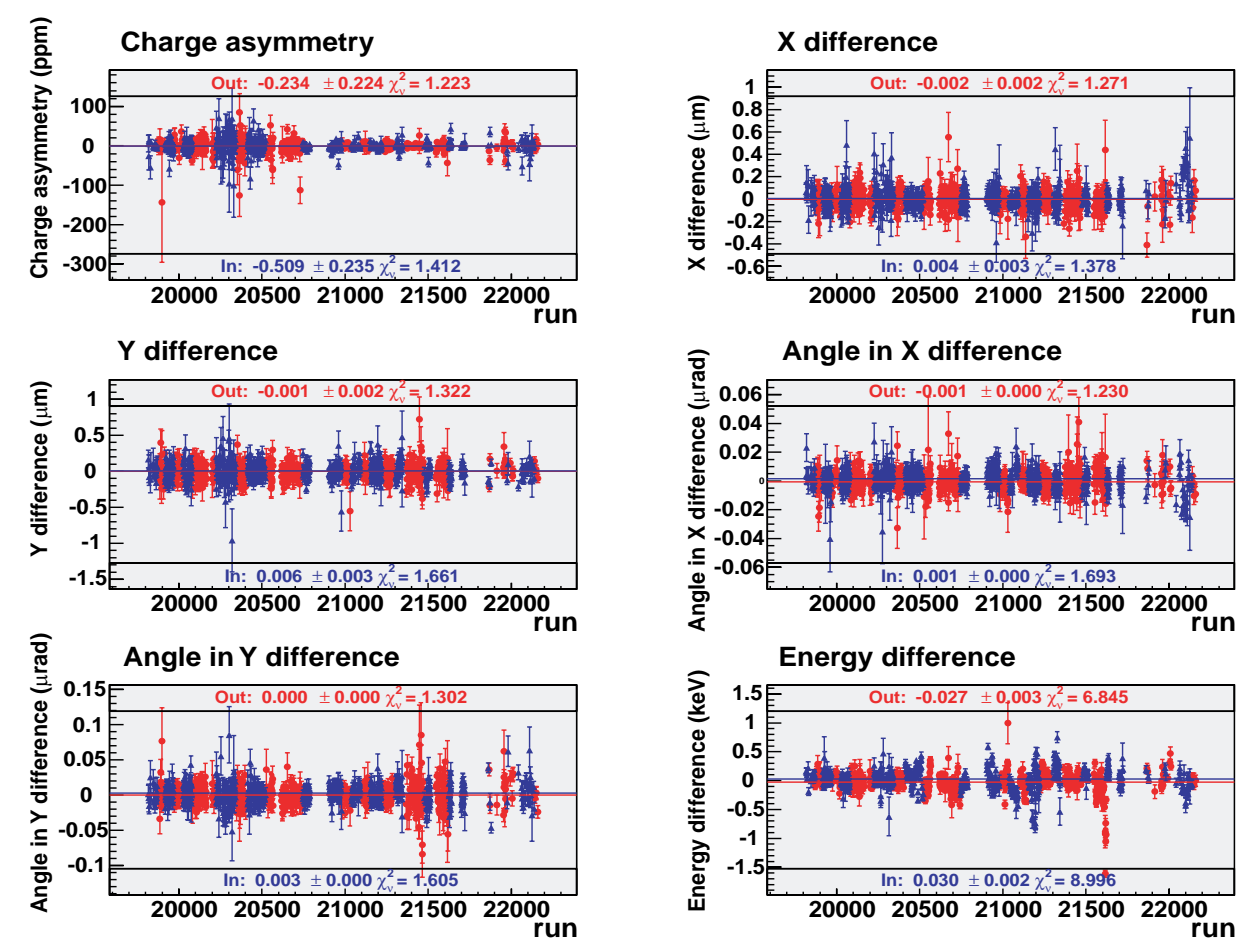

Figure 2.14: Final parity quality results for the $G^{0}$ forward angle measurement. Left to right, and top to bottom, they are charge asymmetry, $\mathrm{x}$ position difference, $\mathrm{y}$ position difference, $\mathrm{x}$ angle difference, y angle difference, and energy difference. The two colors represent the two IHWP states.

order 10's of ppm for the charge asymmetry, and position differences of 100's of nm. The false asymmetry determined from the above parity quality beam is discussed in the analysis section.

\section{$2.3 \quad G^{0}$ Target}

The polarized electron beam is incident on a $20 \mathrm{~cm}$ liquid hydrogen target. The target system can be seen in Fig. 2.15 [65]. It is composed of the cryogenic loop which houses the LH2 target, the target service module which the loop is mounted on, and the target control system.

The cryogenic loop seen in Fig. 2.16 consists of the target manifold and the cryogenic system. The target manifold is constructed from Al-6061 T6, and is connected to the main target hydrogen cell and a helium cell through stainless-Al flanges. The aluminum hydrogen target cell is a cylindrical tube $8 \mathrm{~mm}$ in diameter, and holds 25 liters of liquid hydrogen maintained at $19 \mathrm{~K}$ at 1.7 atm. The thickness of the target in these conditions is $1.44 \mathrm{~g} / \mathrm{cm}^{2}$ which, combined with a beam current of $40 \mu \mathrm{A}$ gives a luminosity of about $2 \times 10^{38} \mathrm{~cm}^{-2} \mathrm{~s}^{-1}$. As seen in Fig. 2.17, the hydrogen 


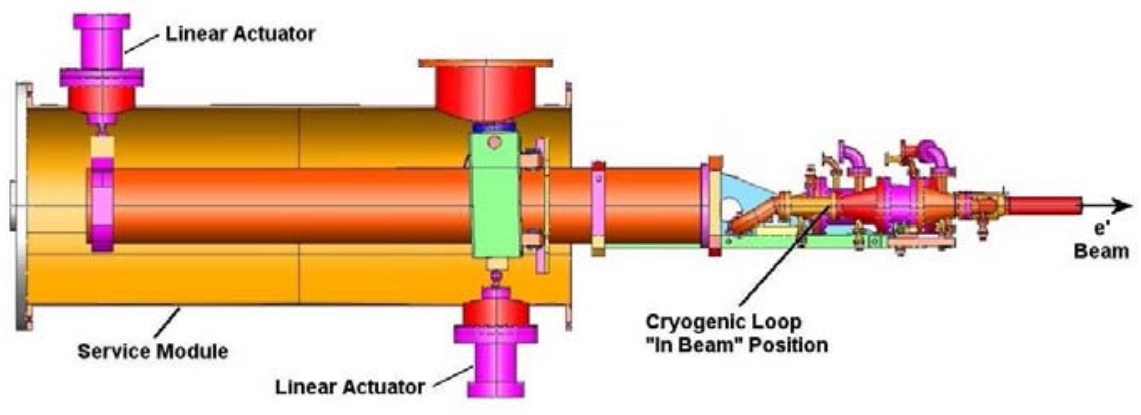

Figure 2.15: $G^{0}$ target

flows in through the inner cone of the hydrogen cell, and exits out between the cone and the outer cell wall. During the first engineering run, the entrance and exit windows of the cell were 0.007"

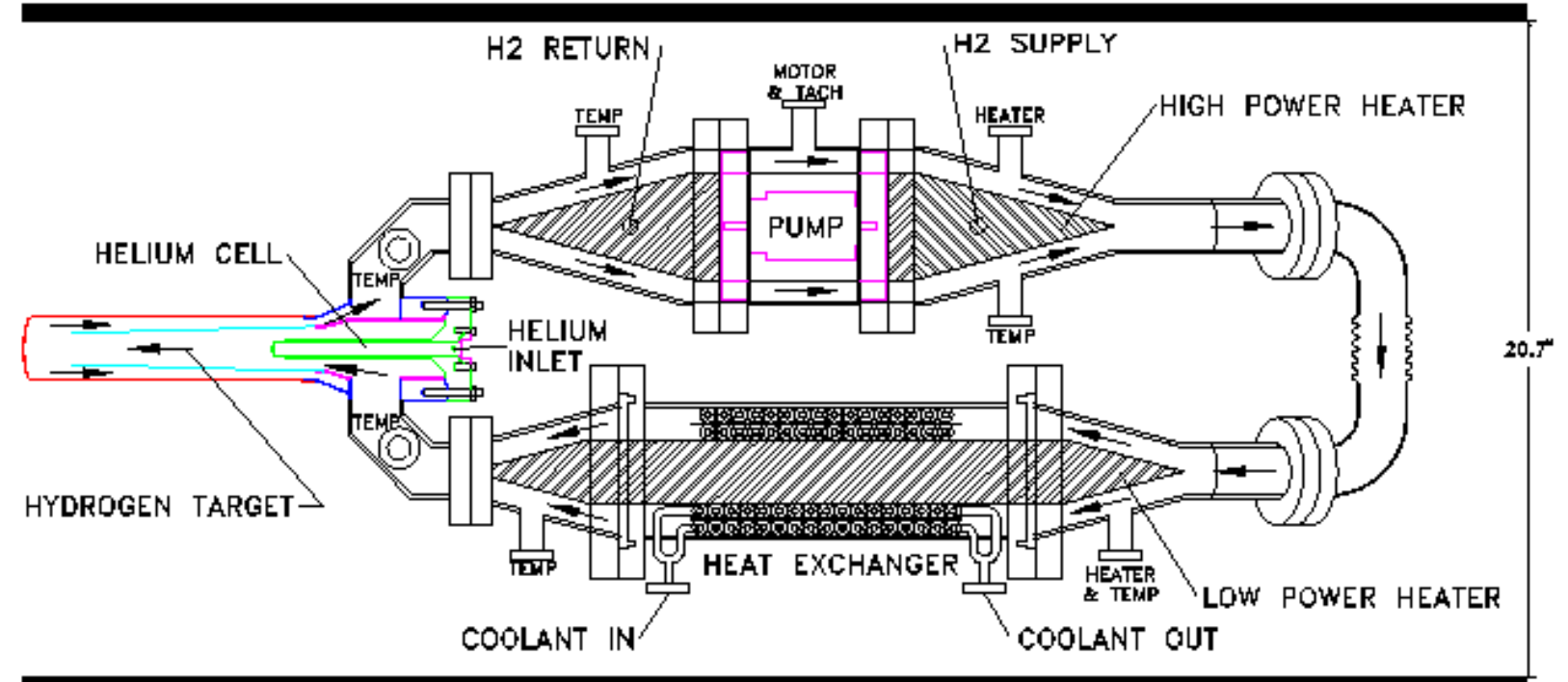

NOTE: The port postitions for electrical and rransducer feedthroughs may be rotated into other planes.

Figure 2.16: $G^{0}$ cryogenic loop

and 0.011 " respectively. However, for the 2nd engineering run and the forward angle production run, the exit window thickness was thinned down to 0.003 " in the central region of the exit window (3/8 in. diameter) in order to reduce the background coming from inelastic photo-production. A $16 \mathrm{~cm}$ long helium cell is located upstream of the hydrogen cell. The exit window of the helium cell is located $20 \mathrm{~cm}$ upstream of the hydrogen cell exit window, and this length defines the liquid 


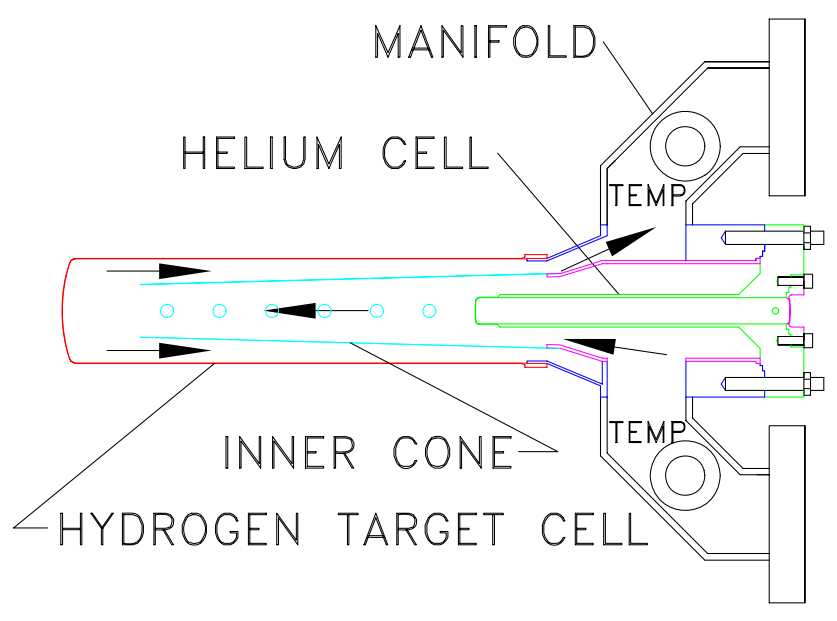

Figure 2.17: $G^{0}$ target manifold

hydrogen target. The exit windows of the two cells through which the beam passes have the same convex shape such that parallel beam drifts (angular changes are relatively much smaller) do not change the beam path-length within the cell. Both cells are maintained at equal pressures of 1.7 atm such that the thin helium exit window will not deform.

In order to keep the density of the hydrogen stable, the temperature of the target is regulated by the target cryogenic loop to be about $19 \mathrm{~K}$ with temperature excursions of about $0.2 \mathrm{~K}$. At 40 $\mu \mathrm{A}$, the energy deposited in the target is about $320 \mathrm{~W}$, whereas the cryogenic loop is designed to be able to remove up to $1000 \mathrm{~W}$ of heat from the liquid hydrogen. On one of the legs of the cryogenic loop, the cryogenic pump is installed (Fig. 2.16) to ensure the high flow-rate of about $8 \mathrm{~m} / \mathrm{s}$ of liquid hydrogen. The pump is a vane-axial design with two impellers in series. The motor is a Barber-Nichols Inc. [66] custom made DC cryogenic motor. A tachometer is installed downstream of the pump to measure the rotation frequency of the pump. The downstream impeller of the pump has a permanent dipole magnet attached to it. When the impeller rotates, a varying magnetic flux through the coil caused by the magnet induces a varying electric field with the same frequency as the rotational frequency of the impeller. During the forward angle production run, the nominal rotational frequency of the pump was $31 \mathrm{~Hz}$.

The $G^{0}$ beam is nominally about 100 microns in both $\mathrm{x}$ and $\mathrm{y}$, which not only boils the target liquid, but could also damage the target cell as it has power densities in the range of $\mathrm{kW} / \mathrm{mm}^{2}$. In order to mitigate this, the beam is rastered uniformly over a larger area. For the $G^{0}$ experiment, 
the raster shape is a square, with side dimensions of $2 \times 2 \mathrm{~mm}^{2}$. The raster design is based on an H-bridge technique, and generates a saw-tooth waveform.

A double coil, counterflow, high power heat exchanger is installed (Fig. 2.16) on the leg opposite to that of the pump to remove the heat deposited in the hydrogen by the beam. The JLab End Station Refrigerator supplies $15 \mathrm{~K} / 12 \mathrm{~atm}$ gaseous helium, which cools the heat exchanger. Since the freezing point of hydrogen is $13.8 \mathrm{~K}$, and the boiling point is $22.21 \mathrm{~K}$ at $1.7 \mathrm{~atm}$, the cryogenic loop must operate in a way that would keep the target temperature stable despite fluctuations in heat load that may occur due to beam intensity changes. To this end, a high powered heater $(\mathrm{HPH})$ is installed near the pump, and is controlled through a Proportional-Integral-Differential (PID) loop to adjust the heat load on the liquid hydrogen. The HPH is made of three independent Ni-Cr alloy ribbon coils, each with a resistance of about $3.5 \Omega$. To assure efficient heat transfer or removal, flow diverters are placed inside the cryogenic loop for the purpose of creating turbulent flow with a high Reynolds number. The Reynolds number for the hydrogen inside the loop was measured to be in excess of $10^{6}$.

The heat exchanger houses a single coil low power heater (LPH) at the upstream end. The LPH was initially installed to mitigate long term temperature fluctuations due to the numerous beam trips the accelerator experience every day. It can output a maximum of $80 \mathrm{~W}$ and worked as part of a feedback loop. However, since the HPH was sufficient in regulating the target temperature, the LPH was never used during the experiment.

In addition to the main liquid hydrogen target used in the experiment, the $G^{0}$ target system also has five other targets used for tests, calibrations, and diagnostics. These targets include the small and large "hole" targets, the aluminum frame target, the flyswatter target, and the tungsten (W) radiator. In order to install the hole targets, the aluminum frame target, and the tungsten radiator, a horizontal aluminum frame is mounted across the target cell manifold upstream of the target cell sitting at the edge of the spectrometer acceptance. The three targets are then installed on the aluminum frame, with the aluminum target being a part of the frame itself. This aluminum frame is used to study the effect of background as well as for the purpose of detector and beam studies. The small and large hole targets are empty $5.46 \mathrm{~mm}$ and $11.05 \mathrm{~mm}$ diameter holes in the target frame which are primarily used to ensure that the beam halo does not strike the target outside 
the helium cell. The flyswatter and the $\mathrm{W}$ targets are used to study and simulate the background produced by the aluminum entrance and exit windows of the hydrogen cell. The $\mathrm{W}$ target is placed $38.5 \mathrm{~cm}$ upstream of the entrance window, and has the same radiation length (thickness) as the liquid hydrogen target. The flyswatter is an aluminum foil target located downstream of the hydrogen cell and mounted on a bendable elbow such that it can be inserted and retracted when needed. However, early in the second engineering run, the bendable elbow malfunctioned, making the flyswatter inoperable.

The target is mounted on the end of a $24 \mathrm{in}$. diameter cylindrical vacuum vessel called the "target service module", and is inserted into the superconducting magnet system in such a way that the target cell is the most downstream target element. Fig. 2.15 shows the target encased within the service module. The service module is connected to the upstream end of the magnet with the cryogenic loop and the target manifold protruding into the magnet cryostat. The service module not only provides mechanical support for the cryogenic loop, but also acts as an interface for electrical lines from vacuum and cryogens to the outside world.

\subsubsection{Target Systematics}

There are several sources of uncertainty associated with the $G^{0}$ target that must be addressed. These include vibrations of the target due to the rotation of the pump, target instabilities from beam trips, target density fluctuations, and global density reductions of the hydrogen. The effect of target vibration due to the pump at the full rotation speed of $75 \mathrm{~Hz}$ is measured to be below 0.01 mm. This is smaller than the natural motion of the beam as well as the size of the beam spot, and is negligible. Furthermore, since the operating pump speed is $31 \mathrm{~Hz}$, which is slightly different from the approximate helicity reversal frequency, this vibration is not expected to be helicity-correlated. The temperature and density instabilities due to a beam trip are mitigated by the PID-operated HPH. Furthermore, events immediately following a beam trip are removed during analysis to reduce the effect of target density and temperature changes, as will be discussed in the analysis section. The temperature excursions are measured to be less than $0.2 \mathrm{~K}$, and the density change is about $0.3 \%$. 


\section{Target Density Fluctuations}

Density fluctuations are random changes in the liquid density driven mainly by fluctuations in beam properties. The mechanism for density fluctuations are complex and are not understood quantitatively. For example, small bubbles may form due to the heating of the hydrogen target, changing the effective target length to fluctuate. The mechanism of target density fluctuations manifests itself by enlarging the asymmetry width. This increased width, of course, translates into a larger statistical uncertainty in the measured asymmetry.

The effect of target density fluctuations is studied by independently varying the raster size, target temperature, and the cryogenic pump rotation speed. The raster is varied to sizes of 0.64 , 2.9, 4, and $9 \mathrm{~mm}^{2}$ at a constant pump speed of $31 \mathrm{~Hz}$, and the LUMI monitor asymmetry width is measured at each setting. The result shows a non-linear behavior of the measured width on the raster size. Although the exact analytical dependence of the width to the raster size is unknown, the above measurements are sufficiently fit with an inverse power law and extrapolated to the operational raster size of $4 \mathrm{~mm}^{2}$. The resulting contribution of target density fluctuations to the asymmetry width is determined to be about $238 \mathrm{ppm}$, which is negligible when added in quadrature to the typical FPD asymmetry width of about $1200 \mathrm{ppm}$. A plot of the width and the extrapolation to the operating point can be seen in Fig. 2.18.

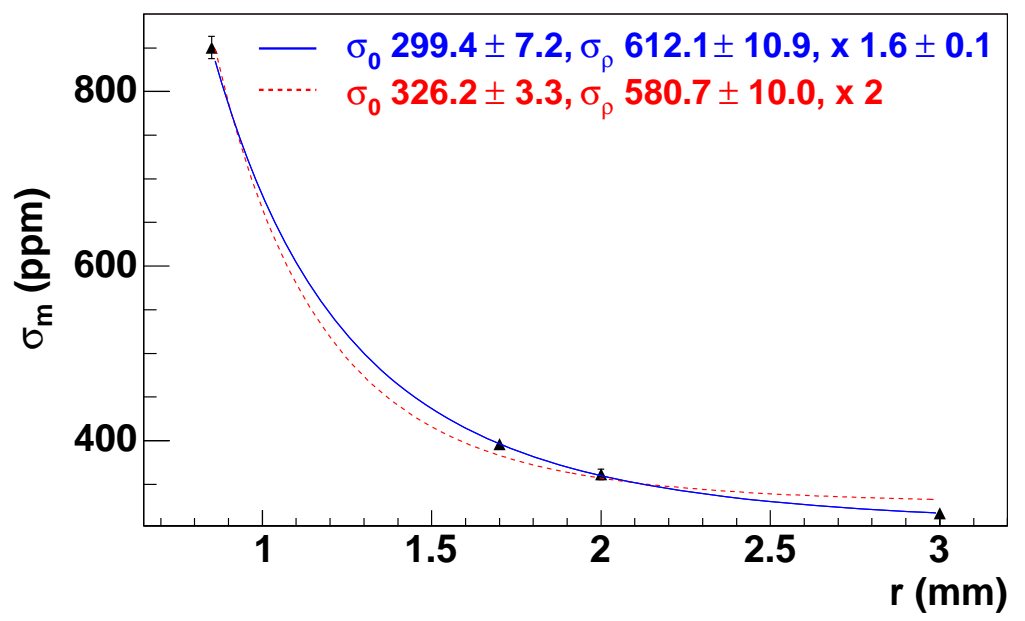

Figure 2.18: FPD asymmetry width as a function of raster size. The width is measured with varying raster sizes, and extrapolated to the operating raster size of $4 \mathrm{~mm}^{2}$. 
The variation of the width is also measured at pump speeds of 31 and $42 \mathrm{~Hz}$. Although there is not enough data to make a model extraction, the widths show a significant drop of about 100 ppm at the higher pump speed. The LUMI widths are also monitored while the target temperature is varied between 18 and $21 \mathrm{~K}$. This resulted in a $3 \%$ change in the widths, which is negligible, especially considering that typical temperature excursions during ordinary experimental conditions is only about $0.2 \mathrm{~K}$.

\section{Target Density Reduction}

Target density reduction refers to the effect where the density of the target is reduced whenever there is beam on target. The result is a reduction in the detector yield, and hence a reduction in the overall statistics accumulated in a given period of time. The study of target density reduction, therefore, is a measure of how the detector yields change as a function of beam current. In order to extract the yield reduction as a function of beam current, while avoiding deadtime corrections, data at the same beam current and different raster sizes are extrapolated to nominal raster size and different beam currents. This approach would keep the deadtime effect the same for each measurement by maintaining the same beam current. Furthermore, the power density of each measurement is the same, since the data are extrapolated to the same raster size of $2 \times 2 m^{2}$. Thus, the yield is directly correlated to only the beam current. A similar study is conducted with the LUMI detectors, but by directly varying the beam current since the (integrating) LUMI detectors are not susceptible to deadtime effects. These measurements are consistent with each other, and show that the overall detector yield reduction at a pump frequency of $31 \mathrm{~Hz}$ is less than $1.5 \%$ at $40 \mu \mathrm{A}$, and $0.4 \%$ for the LUMIs.

\section{$2.4 G^{0}$ Superconducting Magnet System}

The $G^{0}$ Superconducting Magnet System (SMS) [67] is composed of eight azimuthally symmetric octants, forming a toroidal magnetic field in order to bend the forward scattered particles into the detectors. A set of lead and aluminum collimators define the acceptance of the scattered particles. The eight coils are made of superconducting Niobium-Titanium (Ni-Ti) alloys cooled down to $4.5 \mathrm{~K}$ using liquid helium. The toroidal winding is such that the magnetic field lines are drawn 
counter-clockwise looking downstream. The coils are enclosed in a liquid nitrogen shield, which is at a temperature of 90 to $130 \mathrm{~K}$. This shield acts as an intermediary between room temperature and LHe temperature in order to reduce the cooling power needed at $4 \mathrm{~K}$. Vacuum of order $10^{-6}$ Torr is maintained inside the cryostat in order to minimize heat transfer.

\section{SMS Cryogenic System}

The basic cryogenic circuit of the SMS can be seen in Fig. 2.19. 4.5 K liquid helium is used to cool the superconducting coils. There are two separate modes of cycling the helium through the magnet. One is to supply the helium directly to the coils (referred to as forced-flow mode). The other is to send the LHe into a reservoir, which in turn supplies the coils with the coolant (referred to as thermal-siphon mode). The reservoir is in place such that in the event that the LHe supply stops, the coils can still receive a steady flow of helium while the magnet is powered down. During normal running of the experiment, thermal-siphon mode is used, while cooldown from room temperature to superconducting temperature requires running at forced-flow mode. The LN2 circuit also has a reservoir, although the supply of liquid nitrogen is generally more stable. The flow of both helium and nitrogen is regulated using a set of Joule-Thompson (JT) valves. The amount of cryogen in the reservoirs is monitored with a set of level sensors, and the JT valves which are automatically controlled via a PID loop regulates the flow of cryogens to keep both the LHe and LN2 reservoirs full.

The temperature sensors used are platinum resistance thermometers (PT-102), ruthenium-oxide (ROX), and CERNOX sensors. In order to monitor the temperature of the SMS, these temperature sensors are placed at each of the coils, the nitrogen shield, the LHe and LN2 inlets, and the collimators. This gives a global view of the thermal state of the SMS at any given time. A pair of PT and a pair of ROX sensors are attached to each coil. The PT sensors are capable of monitoring temperatures ranging between 30 and $300 \mathrm{~K}$, whereas the ROX can be used for ranges between 4 and $25 \mathrm{~K}$. Therefore, the PT sensors are primarily used for monitoring the temperature of the coils while the SMS is being cooled from $300 \mathrm{~K}$ to about $30 \mathrm{~K}$, while the ROX are used during normal running at $4.5 \mathrm{~K}$. The CERNOX sensors, capable of monitoring temperatures between $4 \mathrm{~K}$ and 300 $\mathrm{K}$, are used to monitor the temperatures of the collimators, and were added after initial testing of 
the magnet system.

\section{SMS Vacuum System}

The cold mass, which includes the coils, is under vacuum in order to minimize heat transfer from the outside world. The vacuum system of the SMS is connected to that of the accelerator, and extends without interruption to the injector. Valves are located at the upstream end of the target and the downstream end of the beam line to allow the vacuum volume associated with the magnet and the target to be isolated from the accelerator. With the valves closed, the magnet and target can be moved out of the beam line. Two $1000 \mathrm{l} / \mathrm{s}$ turbo pumps are installed to keep the magnet under vacuum. One is mounted on the pump-out port below the magnet, and another is placed on the downstream end of the exit beamline. While the magnet is at room temperature, the vacuum is primarily maintained by the turbo pumps at about $10^{-5}$ Torr. When it is cooled to superconducting temperatures, however, the vacuum is cryopumped, and is of order $10^{-6}$ Torr.

A set of pressure gauges are installed to measure the vacuum in the magnet. A cold-cathode gauge is installed at the " 0 " port at the top of the magnet, a thermocouple gauge is located on the "cryobox", and a thermocouple and a cold-cathode gauge are mounted at the bottom of the magnet on the shear-pin port (see Fig. 2.21).

\section{SMS Electrical System}

For the forward angle run, the SMS operates at a current of $5000 \mathrm{~A}$, or at a magnetic field integral of 1.6 Tesla-meters. The total energy stored in the coils and the fields at this current is 6.7 MJ. The SMS electrical circuit can be seen in Fig. 2.20. The current is provided by a Dynapower 8000 A silicon-controlled-rectifier (SCR) [68] based supply, jumpered for $20 \mathrm{~V}$ output. It supports bidirectional power flow allowing the magnet to be both charged from and discharged slowly to the utility power grid. A zero-field current transducer (ZFCT) is used to measure the current supplied to the magnet and to provide feedback to the power supply for current regulation. The current from the power supply is transmitted to the SMS coils with 600 and 1000 MCM (seen in Fig. 2.20) flexible water-cooled cables. These cables are the only components in the SMS power system which

serves as the interconnection between the room-temperature power supply and the superconducting 


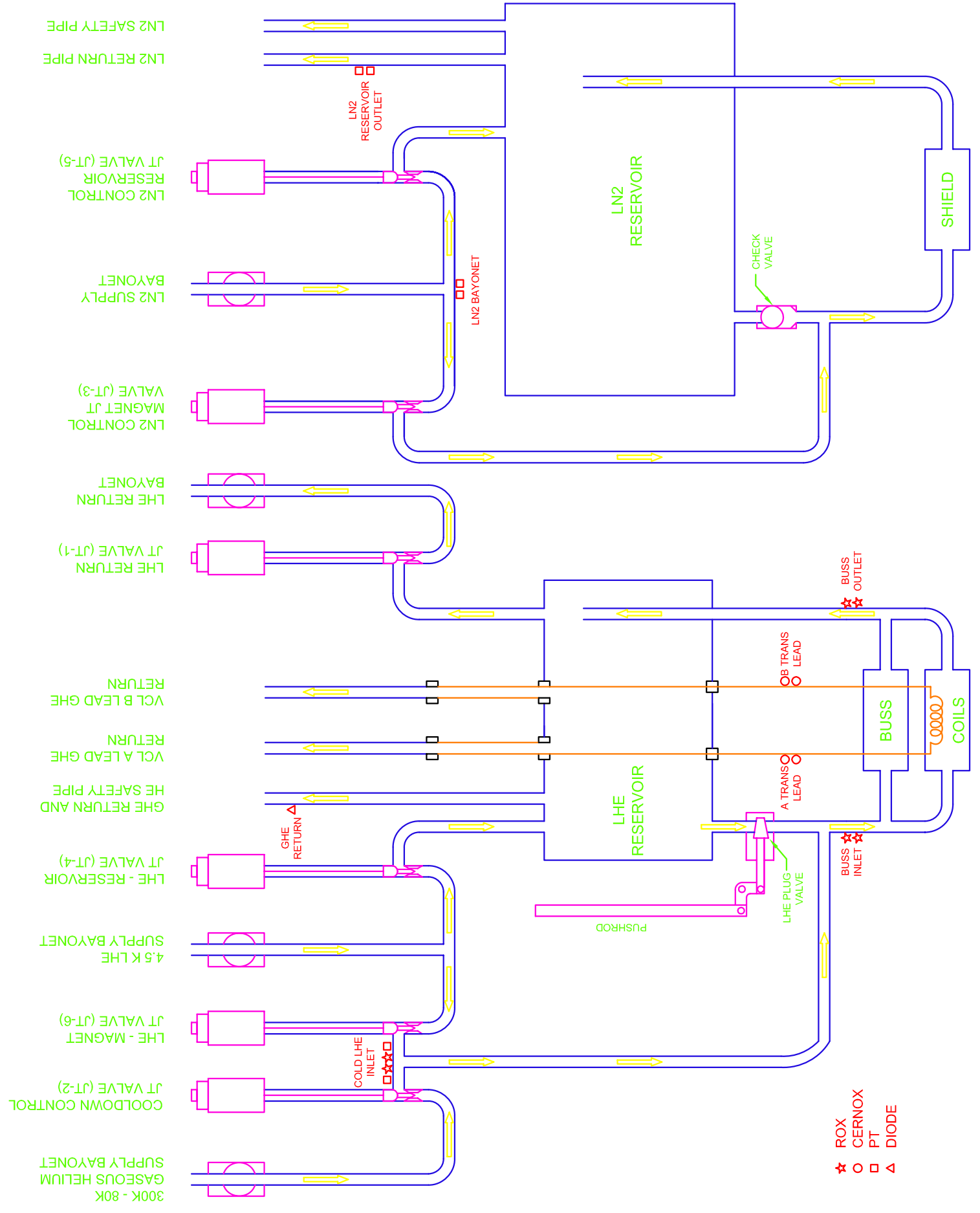

Figure 2.19: $G^{0}$ SMS cryogenic circuit. The cryogenic system is separated into the LN2 circuit which supplies the liquid nitrogen shield with coolant, and the LHe circuit which supplies liquid helium to the superconducting coils. 


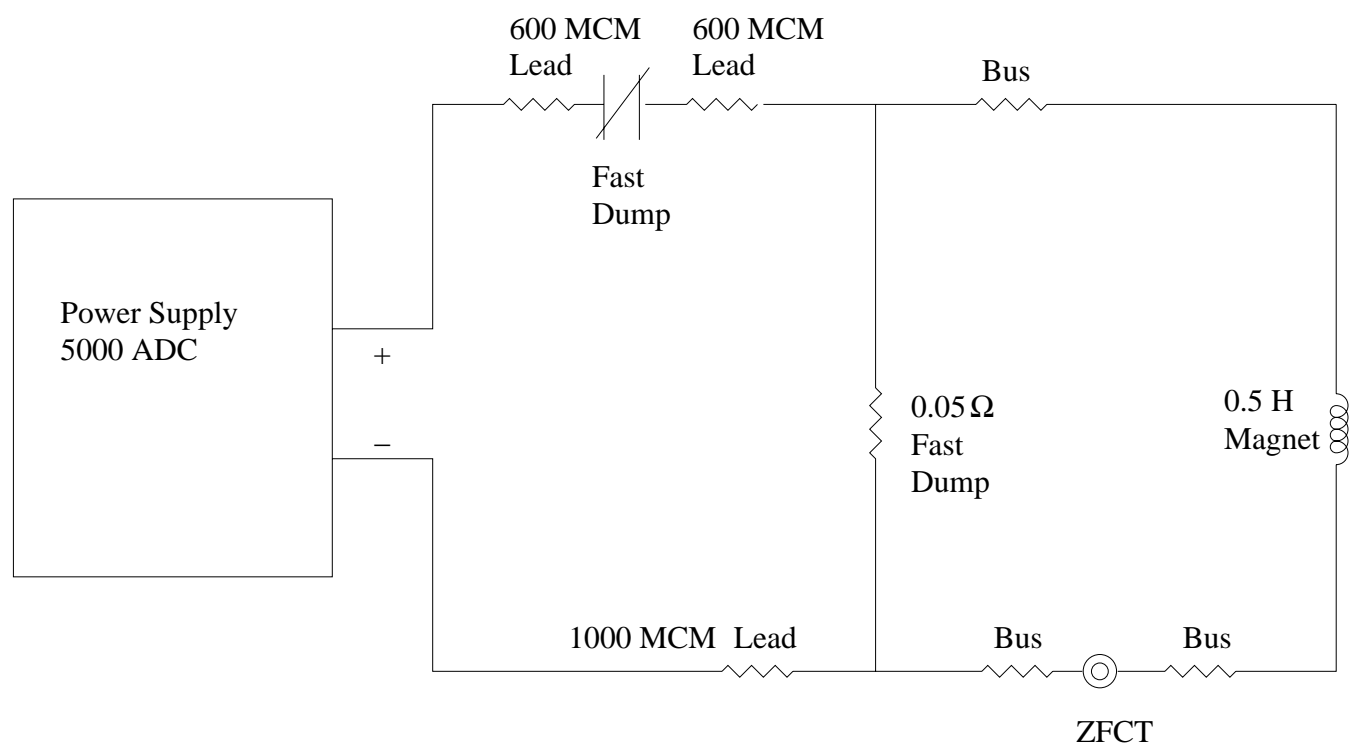

Figure 2.20: $G^{0}$ SMS electrical circuit. A 5000A power supply distributes current through the leads to the SMS coils. In the event of a quench, a circuit breaker cuts the connection to one of the leads, and dissipates the current through a $0.05 \Omega$ resistor.

coils. The $1000 \mathrm{MCM}$ cables run from the power supply to the magnet, while the short $600 \mathrm{MCM}$ cables are used as "jumper" leads between the dump switch, dump resistor, and power supply.

The water-cooled cables are connected to the superconducting buss via vapor cooled leads. These leads are cooled with helium vapor which originates from the LHe reservoir. Thus, the water-cooled (which must be carefully kept above freezing point) and LHe vapor-cooled regions of the leads serve as a gradual transition from room temperature to superconducting temperature.

In the event that the CHL stops supplying helium, the coils have the danger of reverting to a resistive state while still running at $5000 \mathrm{~A}$. In order to avoid this, two separate systems are put in place to remove the stored energy from the coils in this situation. One is the so-called "slow dump", where the current output by the power supply is reversed and the $5000 \mathrm{~A}$ is removed from the coils in about 900 seconds. This mode of removing the energy is used mainly in the case of a power outage or a CHL crash where the reservoir continues to supply helium, but the main JLab coolant supply has ceased. In this mode, the reservoir supply keeps the coils at $4.5 \mathrm{~K}$ long enough that a slower current depletion will not cause thermal shock to the coils. However, in the case the coils revert to a resistive state (a "quench"), the current must be removed immediately. In this case of a "fast dump", the power supply is disconnected from the magnet with a Scheron SA 
high-speed circuit breaker (rated for $6000 \mathrm{~A}$ and $1 \mathrm{kVDC}$ ) and the energy in the magnetic field is dissipated in a $0.5 \Omega$ Post-Glover air-cooled high power resistor. This dump resistor has minimal effect on the circuit (due to its high resistance) when it is in parallel with the superconducting coils. The stored energy of $6.7 \mathrm{MJ}$ is dissipated by the "fast dump" in about 10 seconds. In order to detect when the coils return to a resistive state, a quench detection system is in place. If a non-zero voltage is measured across any of the coils, indicating a quench, the current is "fast dumped". The fast dump of the magnet current, however, generates large eddy currents in the coil cases, in turn producing enough heat to vaporize the liquid helium in the reservoir. The rapid evaporation of the LHe in a fast dump requires a pressure relief mechanism to prevent the LHe system pressure from exceeding 25 psia. An 8 in. diameter parallel plate relief valve is used for this purpose. It should be pointed out, however, a magnet quench occurred only once (during testing), likely due to mechanical relaxation in the superconducting coils or buss. Subsequent fast dumps are a result of electronic failures associated with the control system due to radiation damage.

\section{SMS Control System}

Fig. 2.22 shows the general schematic of the control system. The SMS control system monitors the status of the magnet continuously throughout the run. The system can be roughly separated into the sensor system, the PLC and the temperature controllers, and the control system software.

The sensor system, which includes the temperature sensors, voltage taps, and pressure gauges, continuously read out critical magnet parameters. All temperature and voltage sensor cables are fed through multi-pin Ceramaseal connector ports located on various parts of the magnet as seen in Fig. 2.21. In order to measure the flow rate through the helium vapor-cooled part of the transition cables, a pair of MKS [69] 1599 flow meters are installed, while that of the water-cooled cables are measured by Proteus [70] flow meters. The MKS flow meters are located at the top of the magnet on a rack panel near the magnet end of the leads, while the water flow meters are located at the power supply end of the leads.

The signals from the various sensors and valves are sent to a Direct Logic DL405 programmable logic controller (PLC) by Automation Direct [71], which has an independent CPU and memory to interface with and store readouts from these sensors. The exceptions are the temperature readouts 


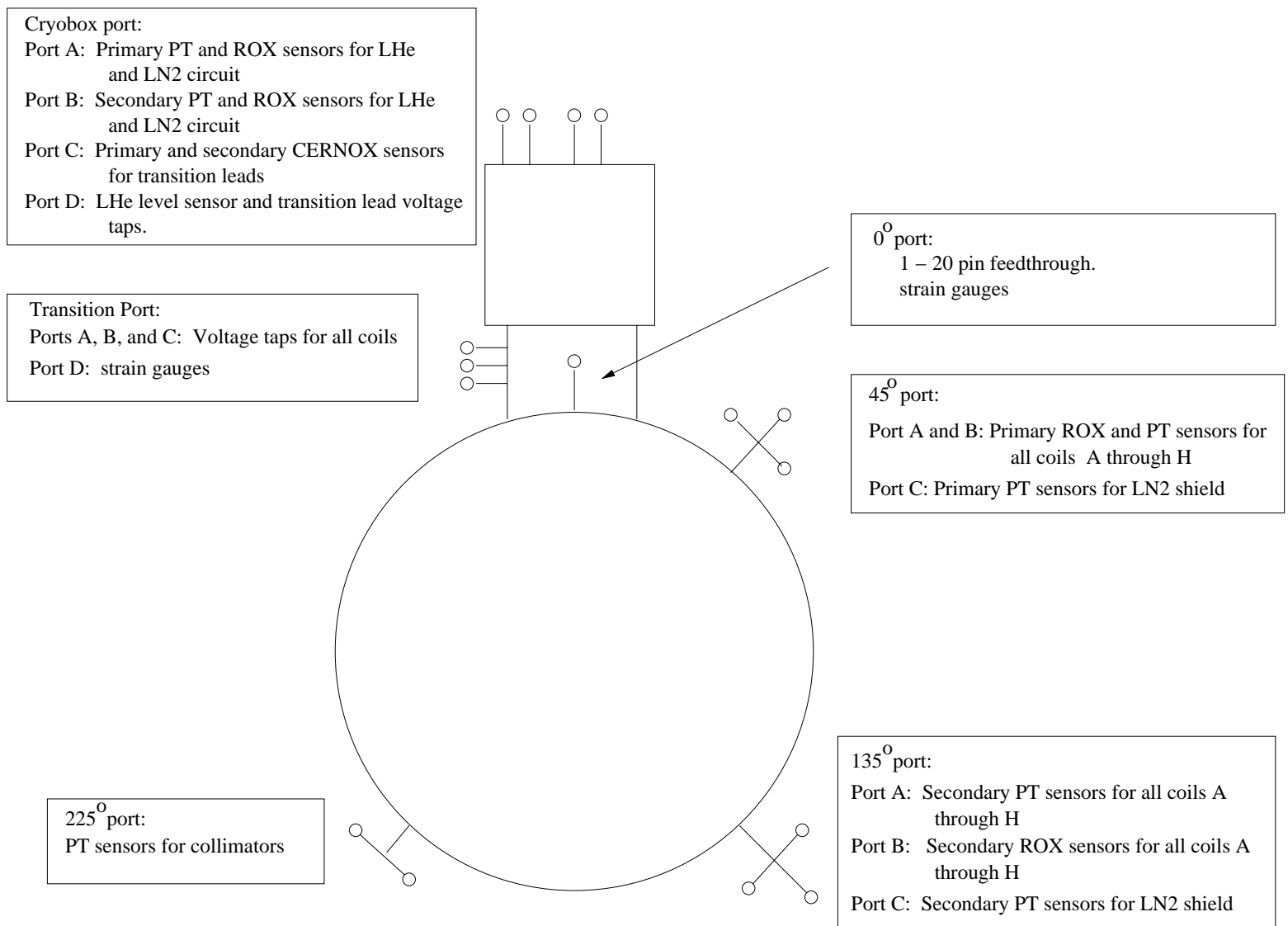

Figure 2.21: Location of feedthrough ports.

which are sent to a set of eight-input temperature controllers manufactured by Lakeshore Inc [72]. The readouts from the PLC and the temperature controllers are then accessed and controlled through a Labview-like control system software called LookoutDirect (National Instruments) which runs on a Windows operating system.

\section{$2.5 \quad G^{0}$ FPD and Electronics}

\section{$G^{0}$ Focal Plane Detectors}

The $G^{0}$ Focal Plane Detectors (FPDs) are 16 sets of double-layered scintillator detectors placed on the focal surface of the SMS. The scintillators are arc shaped such that their elastic proton acceptance is iso- $Q^{2}$. The detector shapes, which take into account the fact that the field integral is larger as one moves closer to the coils, are based on ray-tracing simulations using TOSCA. The 


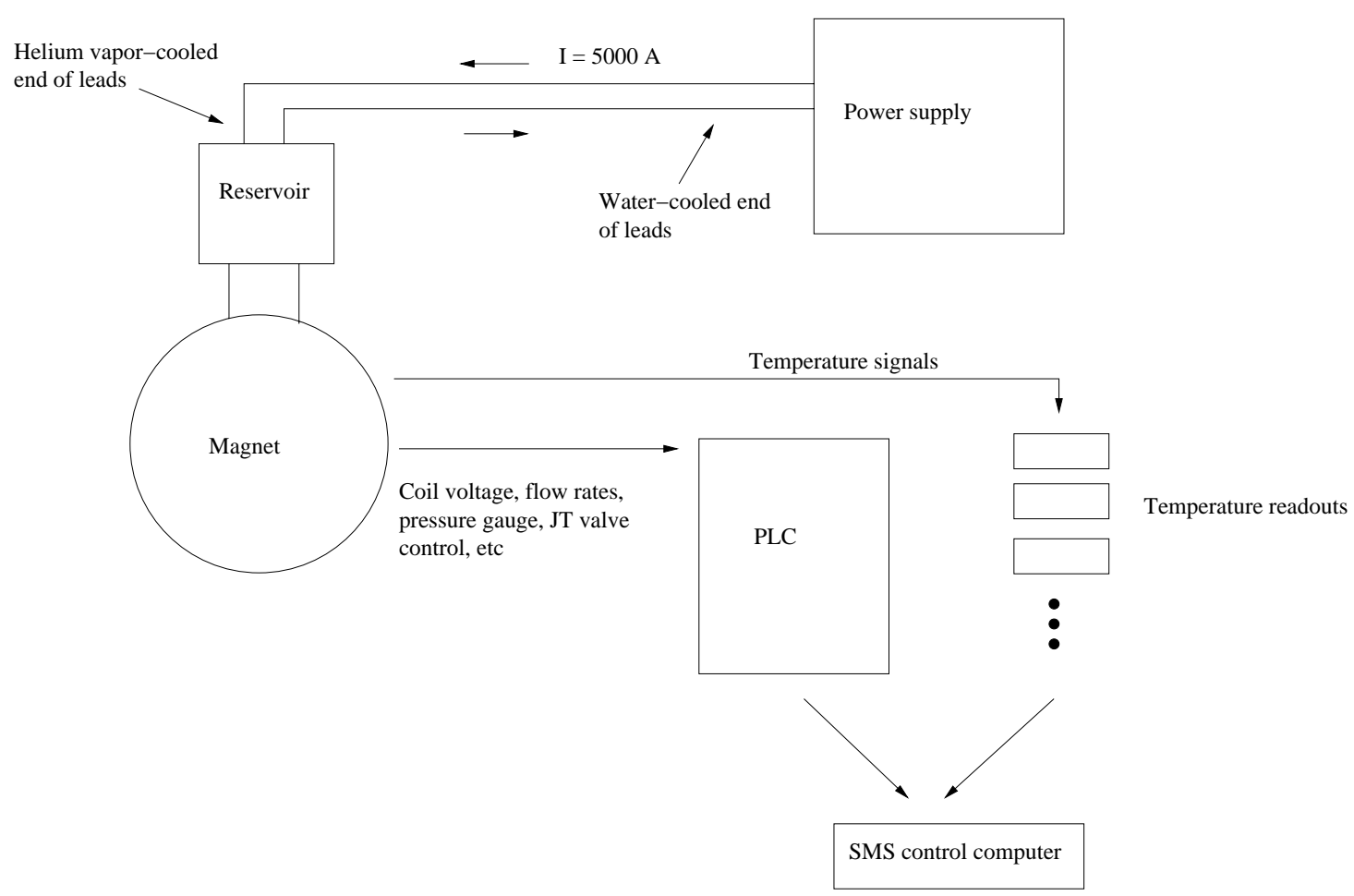

Figure 2.22: SMS control system.

solid angle acceptance of the entire FPD array is about $0.9 \mathrm{sr}$, with an angular acceptance of elastic protons between $52^{\circ}$ and $76^{\circ}$. Fig. 2.23 shows the optics of the $G^{0}$ spectrometer. The recoil protons with low momentum and larger scattering angle are bent more by the magnetic field of the SMS and end up in lower detectors, while the higher momentum protons hit the higher detectors.

The FPDs are placed on an azimuthally symmetric aluminum "ferris wheel" with eight individual octants. There are two different sets of FPDs; one manufactured by the North American collaborators (NA FPDs), and those made by the French collaborators (FR FPDs). There are four octants each of the NA and FR FPDs. The octants are numbered from one to eight, with octant one starting from -22.5 degrees to 22.5 degrees, where 0 degrees is vertically up from the beamline. Looking downstream along the beamline, the octants are counted from one to eight in a counter clockwise manner. Odd numbered octants contain NA FPDs, and the even ones have FR FPDs.

The entire FPD array is sensitive to a kinematic range of $0.12<Q^{2}<1.0(\mathrm{GeV} / \mathrm{c})^{2}$. Detectors 1 through 14 contain $Q^{2}$ bins from 0.12 to $0.41(\mathrm{GeV} / \mathrm{c})^{2}$, whereas detector 15 contains within itself the range from 0.41 to $0.9(\mathrm{GeV} / \mathrm{c})^{2}$. In general, the $Q^{2}$ increases with detector number. However, 


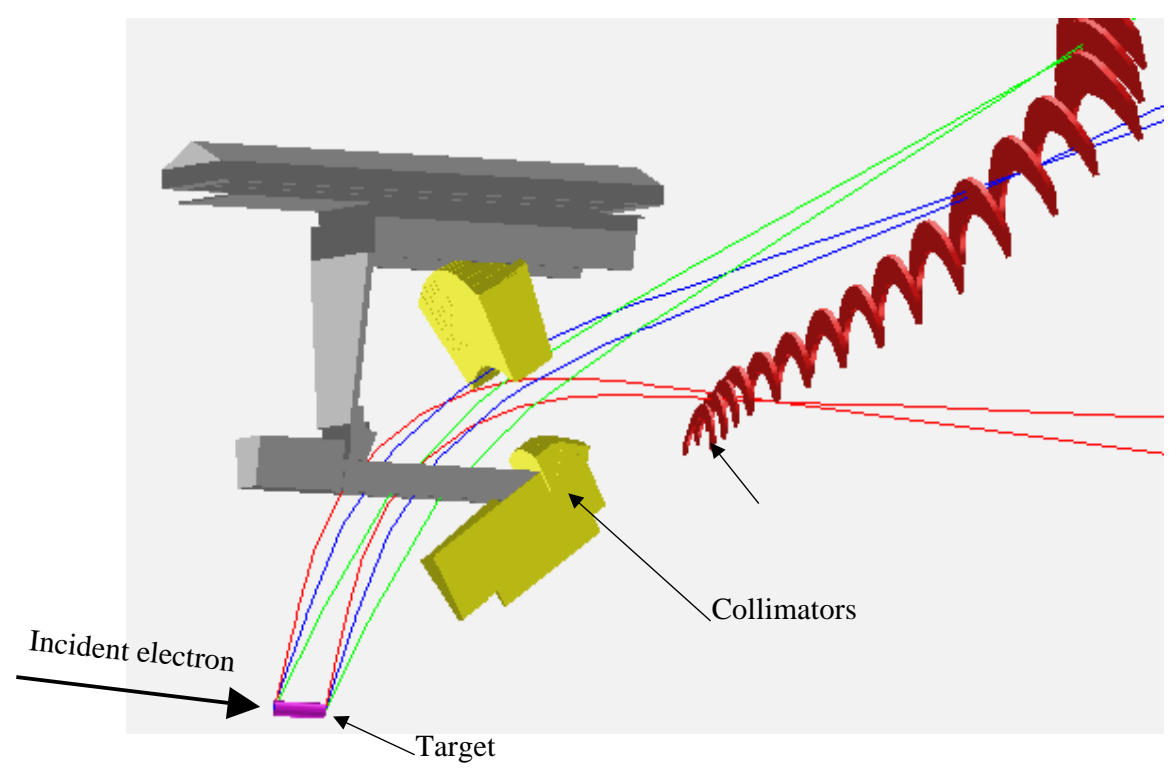

Figure 2.23: Picture of the FPD optics. The red arc-shaped array are the focal plane detectors. The particles scattered from the target are bent by the magnetic field of the SMS to pass through the acceptance-defining collimators (in yellow). The particles hitting different FPDs are separated in $Q^{2}$.

due to the optics of the $G^{0}$ spectrometer, and the fact that the scattering angle decreases as the proton momentum increases, there is a "turn-around" of the $Q^{2}$ distribution at detector 15 . The highest $Q^{2}$ elastic protons ends up in detector 14. Therefore, detector 14 contains two elastic peaks in tof, with the highest $Q^{2}$ elastic protons at a lower tof due to the fact that they arrive at the detector earlier. One peak has an average $Q^{2}$ of $0.41(\mathrm{GeV} / \mathrm{c})^{2}$, and another which has an average $Q^{2}$ of about $1.00(\mathrm{GeV} / \mathrm{c})^{2}$. Detector 16 contains no elastic events in the forward angle experiment, and is used as a check of the background contribution.

The protons deposit anywhere from 2.5 to $9.5 \mathrm{MeV}$ in the scintillators, compared to about $1 \mathrm{MeV}$ for minimum ionizing particles. The number of photo-electrons produced by this energy deposit depends on the scintillator, lightguides, and PMTs that are used in the experiment, and are calculated to be 100 photo-electrons. 
On either end of the arc shaped FPDs are lightguides which transmit the photons to PMTs. There are a total of 8 octants $\times 16 \frac{\text { detectors }}{\text { octant }} \times 2 \frac{\text { layers }}{\text { detector }} \times 2 \frac{P M T s}{\text { layer }}=512$ PMTs. The NA FPDs use Philips [73] XP2262B 12 stage PMTs with zener diode bases, while the FR FPDs use Photonis [74] XP2282B 8 stage PMTs with custom made bases which include amplifiers. Each detector segment is double layered and operate in coincidence (front and back) to improve rejection of backgrounds from neutral particles which might interact in one of the detectors but would be unlikely to interact in both. Each octant is enclosed in an overall light-tight 'box' constructed of an aluminum frame covered with Tedlar.

\section{$G^{0}$ Electronics}

The PMT signals are sent to the time-encoding electronics (TEE) that comprise the main part of the $G^{0}$ electronics [75]. It is responsible for the tof separation of the scattered particles using the passage of the beam pulse through the target as the start signal. A hit is registered by a detector segment when there is a four-fold coincidence event which fires all four PMT's that are connected to the front and back scintillator pairs of the given detector. For any given MPS, or equivalently, helicity state, the TEE counts the number of particles seen in each tof bin. The data are read out during the helicity flip period of $500 \mu$ s at the end of every MPS.

The $\pi^{+}$mesons typically take about 8 ns to reach the FPDs, while elastic protons take an average of about $20 \mathrm{~ns}$. Inelastically scattered protons also populate the tof spectrum between the pion and elastic proton peaks. Although the inelastic protons have lower energy/momentum than their elastic counterparts, these inelastics originate at smaller scattering angles, thus arriving at the detectors earlier.

Both sets of electronics have a feature called the "global buddy" system, whereby detectors that are diametrically opposite to each other are paired as "buddies" and a separate count is kept of hits occurring in a detector's buddy while the electronics of that detector is, itself, busy. Since events missed may be expected to scale like these "buddy events", this system allows corrections for helicity-correlation in deadtime effects.

Although both the NA and FR detectors are set up with time encoding electronics for tof separation, the specific implementation is different between the two. A flowchart of the how the 
data passes through the electronics can be seen in Fig. 2.24.

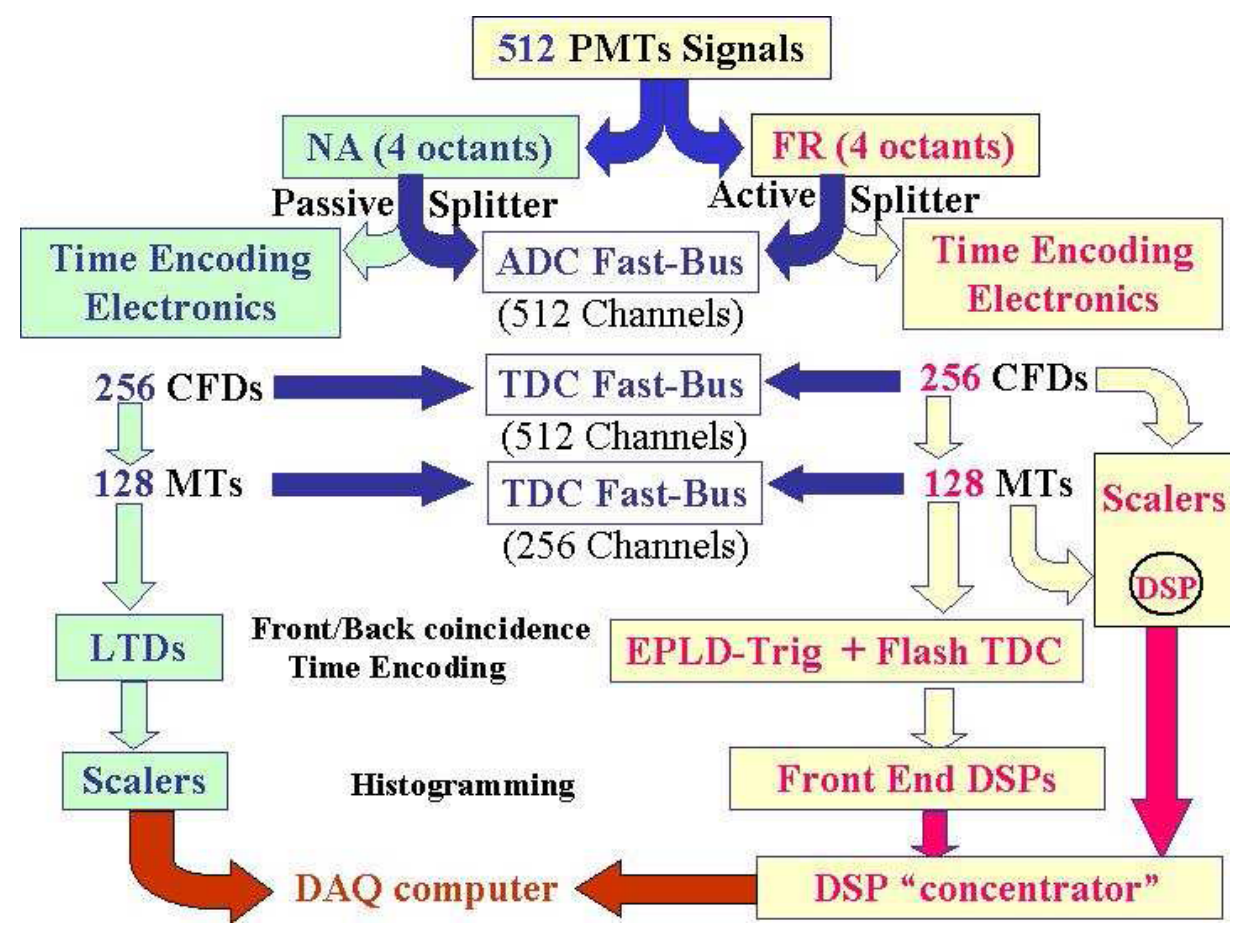

Figure 2.24: Data flowchart through the NA and FR electronics.

For the NA detectors [76], the signals from the PMTs are sent to a constant fraction discriminator (CFD). The CFD has a pulse height threshold which rejects low level noise. Its timing is also independent of the signal amplitude, which makes it suitable for constructing a tof spectrum. The signal is then passed from the CFD to a mean-timer (MT) module that finds the average time of the two PMT signals from each scintillator. The timing of an event is thus independent of where the particle hits along the dimensions of the scintillator. In order for the electronics to create a 32 ns tof distribution, it must know when the beginning of a particular 32 ns cycle begins. To this end, a so-called "Musson Box" was created, which produces two beam-synched signals, the CLK and the $Y_{0}$. Systematic checks of the Musson box signal can be found in Appendix B.1. Both signals are synchronized to the arrival of beam in Hall C, but the CLK is a $499 \mathrm{MHz}$ signal which is used to run the NA shift registers whereas the $Y_{0}$ is a $31 \mathrm{MHz}$ tof start signal. The latter is used to determine the start-time of a $32 \mathrm{~ns}$ time window, and is particularly important in terms of having a stable tof spectrum. The NA FPD signal is then time encoded using a gated clock signal 
to drive the shift registers, which every $32 \mathrm{~ns}$ turns off the master clock for $8 \mathrm{~ns}$, and turns it on for the remaining $24 \mathrm{~ns}$. In the end, the NA tof spectrum monitors $24 \mathrm{~ns}$ out of the $32 \mathrm{~ns}$ beam pulse repetition period, with a tof resolution of $1 \mathrm{~ns}$. This saves the number of scaler channels that must be used, and records hits in tof ranges that are sensitive to the physics of interest. The LTD then sends the data to be read out by scalers, which accumulate the tof histogram for each MPS.

During the first engineering run, the NA electronics showed a large and time-varying differential non-linearity (DNL) effect, where the widths of the time bins would fluctuate. The result is that the PID cuts as well as the dilution of the asymmetry by the background contribution are time dependent and would have required repetitive calibrations. Furthermore, the precision of the $Q^{2}$ determination would have been significantly reduced across all detectors. However, an improved $Y_{0}$ signal has significantly reduced this problem for the second engineering run and the production run. A study of the DNL effect in the NA electronics can be found in Appendix B.2.

In the end, coincidence between the front and back signals are formed to create an overall fourfold coincidence. The 4octants $\times 16$ detectors $=64$ tof spectra are accumulated over each beam pulse and then sent through the latching time digitizer (LTD) which bins each of the 64 signals into 1 ns time bins for input to scalers. Time-sorting through the LTD is achieved through the use of two 16-bit shift registers. The shift registers are synchronized to the $499 \mathrm{MHz}$ CEBAF master clock signal, and are shifted by $\frac{1}{499 \mathrm{MHz}}=2 \mathrm{~ns}$ every micro pulse. The two shift registers are $1 \mathrm{~ns}$ out of phase with each other, and interleaving these two shift registers produces a time resolution of $1 \mathrm{~ns}$ for the tof spectrum for the NA FPD.

The FR electronics uses a fully integrated discriminator, meantimer, time to digital converter (TDC), and histogramming (DMCH - Dedicated MAC Channel) with 16 channels per module which can serve up to 16 scintillators (eight FPD segments). The DMCH board includes programmable logical devices (EPLD-Trig) to detect coincidence hits between front and back scintillators. The arrival time of a hit is encoded by a custom numerical time encoder ASIC (Application Specific Integrated Circuit), a 9-channel $\times 128$ bin flash TDCs which by design has 0.25 ns resolution. As in the NA case, the tof histogramming requires the knowledge of the start time. Therefore, the ASIC is synchronized to the $Y_{0}$ signal. The TDCs distribute data to four front-end Digital Signal Processors (DSPs) where tof spectra are accumulated in different memory registers associated with 
each detector, from which the spectra are read out after the MPS. Compared to the NA electronics, the FR tof spectrum contains 128 bins, or 0.25 ns bin widths, over the 32 ns micro pulse window.

The FR electronics also feature the "differential buddy" system. Whereas the global buddy provides the overall number of counts integrated over an MPS, the differential buddy builds a tof spectrum of these counts allowing for deadtime corrections to be applied on a time bin-by-time bin basis.

The DMCH module can support a daughter-card, which carries 104 scaler channels. These scalers count both "direct CFD" and "direct MT" hits, where CFD signals are counted ignoring the other CFD of the same scintillator, and MT signals are counted without considering the other MT of the same front/back pair. Furthermore, this daughter-card is responsible for implementing the global buddy system in the FR electronics. Finally, "single CFD" events, which are CFD signals only when the related MT is not triggered due to singles loss, are recorded. The single CFD is useful in determining the deadtime in the MT due to single events.

Overall, the implementation of the TEE was successful, although there were instances where they would malfunction. The specific issues affecting either electronics can be found in Appendix B.3 where studies performed to determine detector efficiency are described. In addition to the time encoding electronics, auxiliary Fastbus electronics with a data acquisition rate of about $1 \mathrm{kHz}$ is used as a monitoring sub-system. ADC and TDC modules collecting signals event-by-event for a small fraction of micro pulses, and are common to both electronics. Although only a small fraction of the $G^{0}$ beam pulse is monitored, the tof resolution available from these electronics is $50 \mathrm{ps}$. The use of these electronics is mainly for diagnostic purposes, including the monitoring of deadtime, single hits, $Q^{2}$ determination, and calibrating the time encoding electronics. The effects of single events on deadtime as well as $Q^{2}$ determination are both explained in the analysis section. As seen in Fig. 2.24, the NA and FR TEE use different splitters to accommodate different signal sizes coming from the different tube/amplifier configurations. The result is that the fractional amplitude of signals sent to the TEE is $2 / 3$ for the NA, and 1 for the FR. The Fastbus TDCs receive the signals processed by both the CFDs and meantimers of the TEE. 


\section{Chapter 3}

\section{Analysis}

Once the run is complete and the data are obtained, the raw asymmetry must be corrected for various systematics in order to determine the final physics asymmetry and the strange form factors. These include beam leakage, deadtime, linear regression, background asymmetry, polarization corrections, and radiative corrections. In addition to these corrections, conditions are imposed during data selection in order to removed parts of the data compromised by malfunctioning equipment. This chapter will describe the basic function of the $G^{0}$ analysis software, data selection, the corrections made to the data, and the false asymmetry contributions of each correction to the measured asymmetry.

\section{1 $G^{0}$ Analysis software}

Once the DAQ outputs the raw data, it can be replayed/analyzed by the $G^{0}$ analysis software (called g0analysis), which is written using the $\mathrm{C}++/$ ROOT [77] physics analysis package. The data are segmented into roughly hour-long runs which are assigned individual run-numbers.

\subsubsection{ROOT files and the Database}

The g0analysis software calculates various parameters for each run. All detector and beam data are calculated MPS-by-MPS, while helicity-correlated parameters such as asymmetries and HC beam parameters are calculated quartet-by-quartet. The MPS-by-MPS and quartet-by-quartet data are stored in ROOT files. Each run outputs one ROOT file, from which any parameter calculated by g0analysis can be extracted on an MPS-by-MPS or quartet-by-quartet basis. 
However, for the purpose of any particular run, the values of interest are the average asymmetry as well as the cumulative yield over each run. These average values are stored in a MySQL (Structured Query Language) database [78]. The database is set up to store two types of information. The first is the analysis results. These include values such as average detector yields and asymmetries, beam parameters, number of MPS and quartets in the run, and values needed to apply corrections to the raw measured asymmetry. The second type of data involve values that describe the conditions under which the run is taken. These may include the magnet current, target temperatures, detector/electronics calibrations, and all other parameters that characterize the run-conditions. These run-by-run average values can then be extracted from the database, and combined to give the average and cumulative values of each parameter over the entire forward angle production run.

In order to avoid biasing the asymmetry measurements, the g0analysis code is designed to blind all calculated values. A multiplicative blinding factor in the range $0.75-1.25$ is applied to all asymmetries before the database is filled. This blinding factor is not removed until all corrections to the asymmetry are completed.

\subsubsection{Data Selection}

There are a few cuts applied to the raw data in order to assure data quality. These include,

\section{Magnet current}

2. Target temperature

3. Electronics malfunction

4. Beam trip cut

5. Parity quality cut

Most of these cuts are applied in order to remove parts of the data where one or more experimental subsystem are not functioning properly. Of the 922 hours of data on tape, $14 \%$ were cut due to cuts 1 through 4 prior to analysis, and another $11 \%$ of the remaining data were removed due to parity quality considerations. The mean value of the LH2 target temperature is cut to be between 
17.0 and $22.0 \mathrm{~K}$, while the magnet current must be between 5000 and $5003.5 \mathrm{~A}$ (the power supply regulates current at $0.01 \%$ ). Electronics malfunctions where the electronics would crash or count erroneous rates (see Appendix B.3) are removed from the data. Instances where the electron beam would cease due to beam trips must also be removed from the data. Since a finite amount of time is necessary to ramp the beam current back up to $40 \mu \mathrm{A}$, a wait-time of 500 MPS (about $17 \mathrm{~s}$ ) is used, where data within 500 MPS from the time the beam starts to ramp up are ignored. This wait-time serves not only to remove data where the beam is below $40 \mu \mathrm{A}$, but also to wait for target density fluctuations from beam current variations to settle before analysis resumes. An additional parity quality beam cut is applied where all runs that are outside of the $4 \sigma$ of the specifications of parity quality (see Section 2.2) are to be ignored.

The cuts are applied when the raw data are replayed using g0analysis. If a single MPS within a quartet fails the cut, the entire quartet is dropped. In addition to applying the various cuts to determine the "good" dataset, the g0analysis software also applies deadtime corrections to the measured elastic proton asymmetry and calculates the slopes necessary to correct for false asymmetries from HC beam parameters. The data are replayed twice. Once to determine the parameters necessary to apply corrections to the asymmetries, and another time to apply the corrections themselves. The resulting dataset includes $7.64 \times 10^{7} \mathrm{MPS}$, or $100.88 \mathrm{C}$ of parity quality data.

\subsection{Raw Proton Asymmetry}

Once the "good" dataset is defined, the detector yield for each time bin is defined as the measured number of detector rate normalized to the beam charge

$$
Y(t)=\frac{R(t)}{Q}
$$

where $Y(t)$ is the yield, $R(t)$ is the particle rate in time bin $t$, and $Q$ is the beam charge. The yield is measured for each time bin for both NA (1 ns time bins) and FR ( $0.25 \mathrm{~ns}$ time bins) detectors. 
The asymmetry is then calculated quartet-by-quartet

$$
\begin{aligned}
A^{a}(t) & =\frac{Y_{1}^{+}(t)+Y_{4}^{+}(t)-Y_{2}^{-}(t)-Y_{3}^{-}(t)}{Y_{1}^{+}(t)+Y_{2}^{-}(t)+Y_{3}^{-}(t)+Y_{4}^{+}(t)} \\
A^{b}(t) & =\frac{Y_{2}^{+}(t)+Y_{3}^{+}(t)-Y_{1}^{-}(t)-Y_{4}^{-}(t)}{Y_{1}^{-}(t)+Y_{2}^{+}(t)+Y_{3}^{+}(t)+Y_{4}^{-}(t)}
\end{aligned}
$$

where superscripts $a$ and $b$ indicate the two types of quartets that can be formed depending on the helicity state of the first MPS, +(-) refer to the positive(negative) helicity states, and indices 1 through 4 indicate the four MPS within a quartet. The asymmetries and yields for each time bin are averaged over the entire run. Since the dataset is segmented into roughly 1 hour runs, the asymmetries must be statistically combined over the entire production run. The average raw measured asymmetry for each time bin over the entire 700 hours of data is calculated by weighing the run-by-run asymmetry against the statistical uncertainty [79]

$$
<A_{\text {meas }}(t)>_{\text {all }}=\frac{\sum_{\text {runs }}\left(\frac{A_{\text {meas }}(t)}{\sigma_{A_{\text {meas }}}(t)}\right)^{2}}{\sum_{\text {runs }}\left(\frac{1}{\sigma_{A_{\text {meas }}}(t)}\right)^{2}}
$$

where $\left\langle A_{\text {meas }}(t)>\right.$ is the average asymmetry over the entire production run for each time bin, and $A_{\text {meas }}(t)$ and $\sigma_{A_{\text {meas }}}(t)$ denote the average asymmetry and uncertainty in a time bin for one run. The corresponding statistical error for each time bin over the entire run is

$$
<\sigma_{A_{\text {meas }}}(t)>_{\text {all }}=\frac{1}{\Sigma_{\text {runs }}\left(\frac{1}{\sigma_{A_{\text {meas }}}(t)}\right)^{2}}
$$

where $<\sigma_{A_{\text {meas }}}(t)>_{\text {all }}$ is the statistical uncertainty on $\left\langle A_{\text {meas }}(t)>_{\text {all }}\right.$. The result is a tof spectrum with a normalized yield and an asymmetry associated with each time bin. A sample tof spectrum can be seen in Fig. 3.1 The spectrum shows a clear separation between the pion and the elastic protons due to the fact that the more massive protons move more slowly and thus reach the detectors later compared to the pions. However, a slowly varying inelastic peak is seen to be leaking into the elastic proton peak. This will be addressed further in Section 3.8.

In order to extract the elastic proton asymmetry from the tof spectrum, a set of cuts are defined based on the type of scattering. The asymmetry is then integrated over the elastic proton cut by 


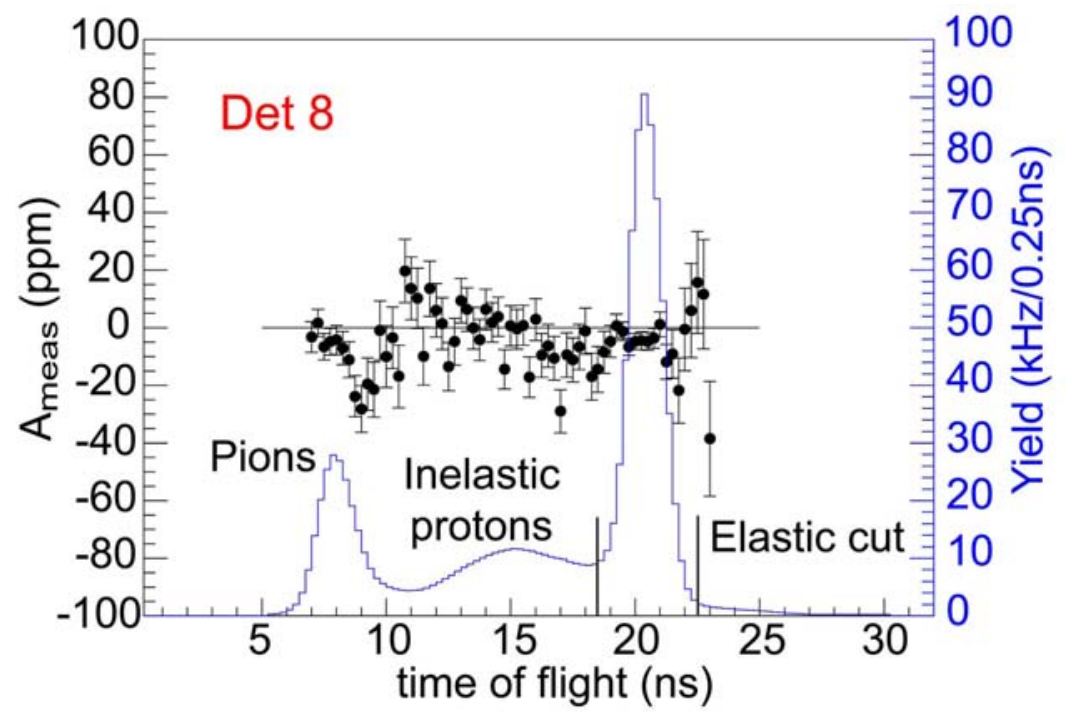

Figure 3.1: Sample tof spectra for octant 2 detector 8. The black points represent the asymmetries measured in each time bin for the entire 700 hours of data-taking. The blue curve is the yield. The first (left-most) peak in the yield curve shows the pions, the right-most peak is the elastic protons, and the small peak between the pions and the elastic protons is the inelastic protons.

weighing against the measured yield, $Y_{\text {meas }}$

$$
A_{\text {proton }}=\frac{\int<A_{\text {meas }}(t)>_{\text {all }} Y_{\text {meas }}(t)}{\int Y_{\text {meas }}(t)}
$$

where $Y_{\text {meas }}(t)$ is the measured yield per time bin, and $A_{\text {proton }}$ is the measured elastic proton asymmetry for the forward angle production run. This is the raw measured asymmetry uncorrected for any systematic effects. The statistical uncertainty is determined from counting statistics

$$
\sigma_{A_{\text {proton }}}=\frac{\sigma_{A_{\text {qrt }}}}{\sqrt{N_{\text {qrt }}}} \approx \frac{1}{\sqrt{N_{\text {proton }}}}
$$

where $\sigma_{A_{q r t}}$ is the statistical width of the asymmetry distribution, $N_{q r t}$ is the number of quartets, and $N_{\text {proton }}$ is the number of protons. Note that the statistical uncertainty calculated in Eqn. 3.5 and from counting statistics in Eqn. 3.7 are equivalent for statistical distributions. However, the 
error measured from the asymmetry width is usually larger than the width from counting statistics due to various systematic effects. In the case of $G^{0}$, the approximation in Eqn. 3.7 is seen to give similar results when one compares the two calculations. Fig. 3.2 shows the counting statistics ratio, defined to be the ratio between the measured width and the width from counting statistics. The width from counting statistics seen here is corrected for deadtime effects. In principle, in a perfect statistical distribution, the ratio would be equal to one. The ratio in the figure show agreement between the two calculations to within a few \% level with the exception of detectors 13 and 14 of octants 4 and 8 . This is thought to be a result of jitters in the elastic proton cut as well as instabilities in the $Y_{0}$. When the same study is performed using the entire tof spectrum, which is less sensitive to jitters in timing, the large excursion in these detectors disappear. In the end, counting statistics is used for the statistical uncertainty, and all other sources of error are included in the systematic uncertainty.

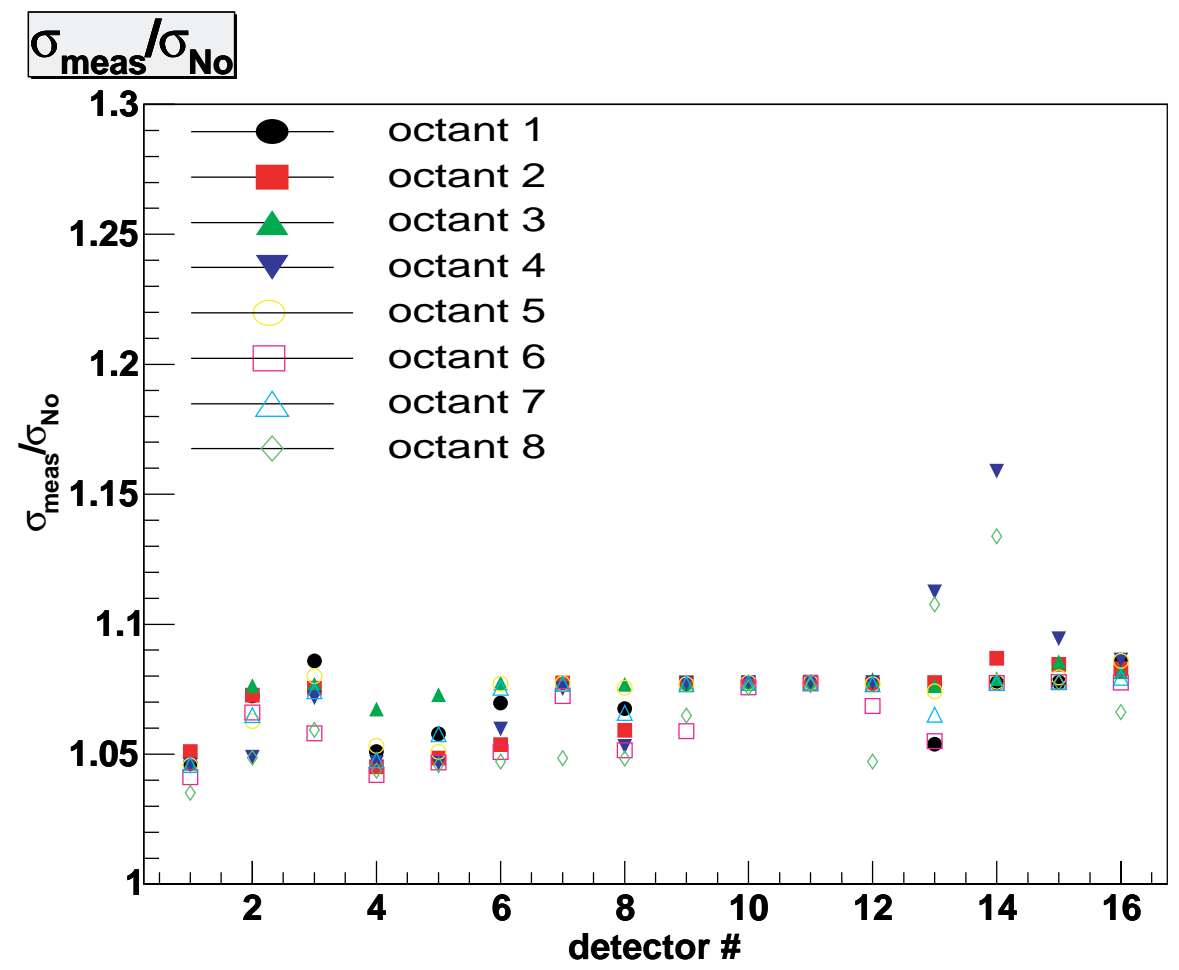

Figure 3.2: Ratio of measured asymmetry width $\left(\sigma_{\text {meas }}\right)$ and the width calculated from counting statistics $\left(\sigma_{N_{0}}\right), \sigma_{N_{0}}$ is corrected for deadtime effects. 


\section{$3.3 \quad$ G0GEANT simulation}

One of the tools used extensively in the analysis of the $G^{0}$ data is the GEANT3 simulation code. The physics behind the experiment, namely elastic e-p scattering, can be simulated using this code. The caveat is that it cannot simulate the asymmetry since the neutral weak form factor is apriori not known. However, observables such as cross sections of primary and secondary scattering, processes such as bremsstrahlung, multiple scattering, and higher order radiative effects, to name a few, can all be simulated by inserting/adding the appropriate code into GEANT3. These processes and observables can then be applied to $G^{0}$ by including the geometry of the experiment as input to the code. The result is a GEANT3-based simulation specific to $G^{0}$, called G0GEANT. The code is written in Fortran, and is used extensively for analysis purposes, but particularly for cases where it is important to know the kinematics of the particles being detected. These include $Q^{2}$ determination, background correction, and radiative corrections. Inelastic particle scattering generators are used extensively to determine what particles are contributing to the background rate, what kinematic range they are coming from, and from what physical processes they originate. This will be addressed further in the discussion of background corrections in Section 3.8. Radiative correction effects are determined by simulating how the elastic cross section change as higher order scattering processes are included in the simulation. This will be addressed in the discussion of radiative corrections in Section 3.10 .

The code is simplified such that the user can set flags as inputs when deciding whether to include certain physical processes, or to include/exclude certain kinematic regions of the experiment. Furthermore, certain geometries and objects in the experiment can be made sensitive to particle hits and turned into detectors, in order to determine the rate of particles hitting these objects. The simulation can be executed in either an interactive or a non-interactive mode. In the interactive mode, it is possible to visually track the trajectory of scattered particles to see whether certain particles are scattering off of secondary objects before hitting the FPDs. Fig. 3.3 shows an example of this particle tracking.

As a vital check of the code, the elastic proton rate in each detector is simulated. Fig. 3.4 shows a comparison of the rates between simulation and data. As will be discussed in the next section, the current through the $G^{0}$ magnet during the experiment is determined to be about 5015 


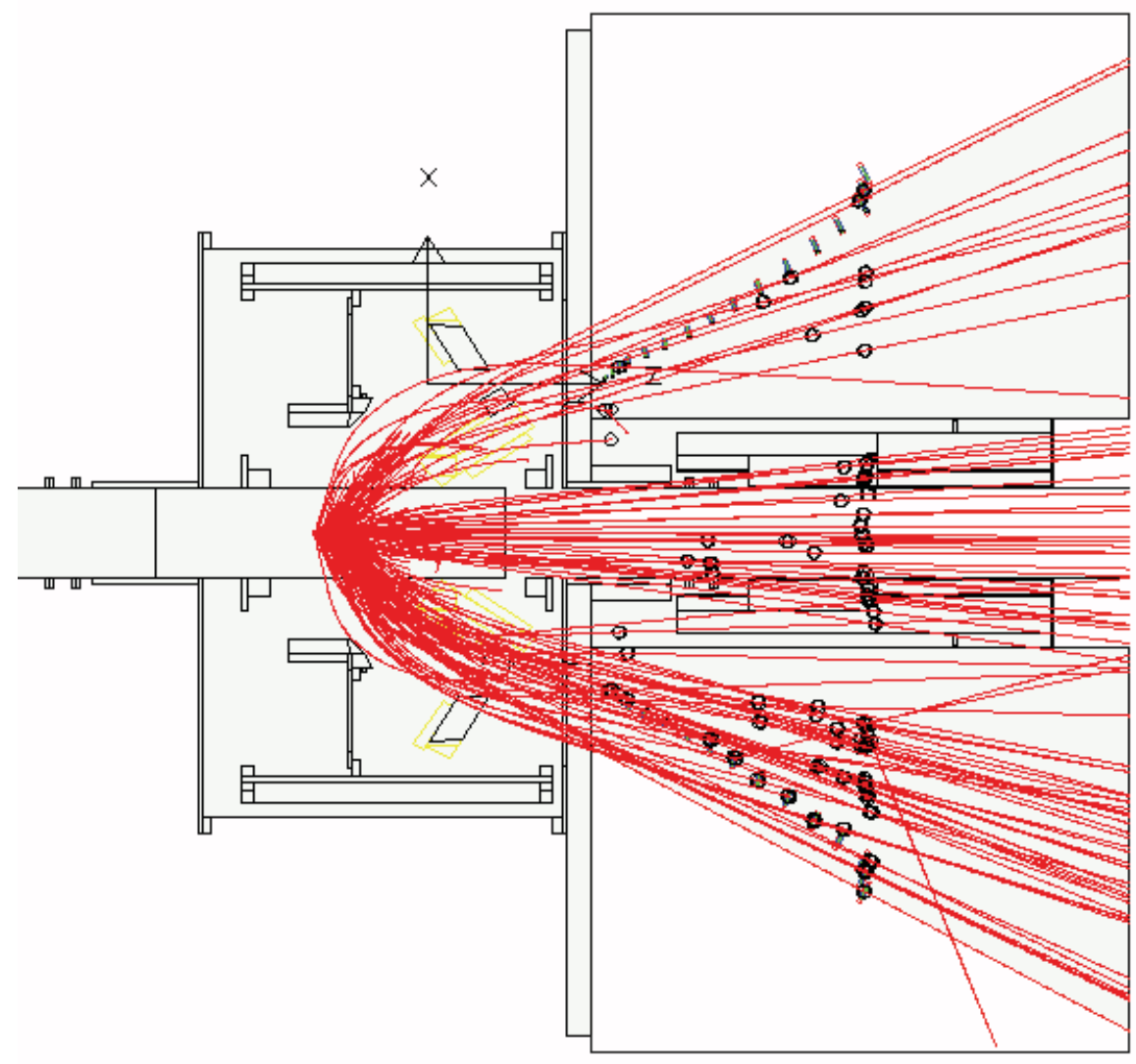

Figure 3.3: Tracking of elastic proton scattering through the G0GEANT simulation projected on the vertical plane. Circles show where the protons are hitting the FPDs. FPDs can be seen for octants 1 and 5. Where there are circles seemingly hitting nothing are actually FPD hits in octants that are out of plane. This particular simulation is for $0.5<Q^{2}<1.0 \mathrm{GeV} / \mathrm{c}^{2}$.

A instead of the nominal 5000 A.

\section{$3.4 Q^{2}$ Determination}

In order to determine the central $Q^{2}$ value of each detector, one first needs to determine the magnetic field produced by the SMS. Two methods were devised, and both were performed using the FR octants due to their greater resolution over the NA octants.

One method is to observe the elastic proton rate in detectors 15 and 16 . At the nominal current of $5000 \mathrm{~A}$, detector 16 should see no elastic rates. However, by varying the magnet current, the focusing of the spectrometer magnet can be altered to bring detector 16 into the elastic acceptance. 


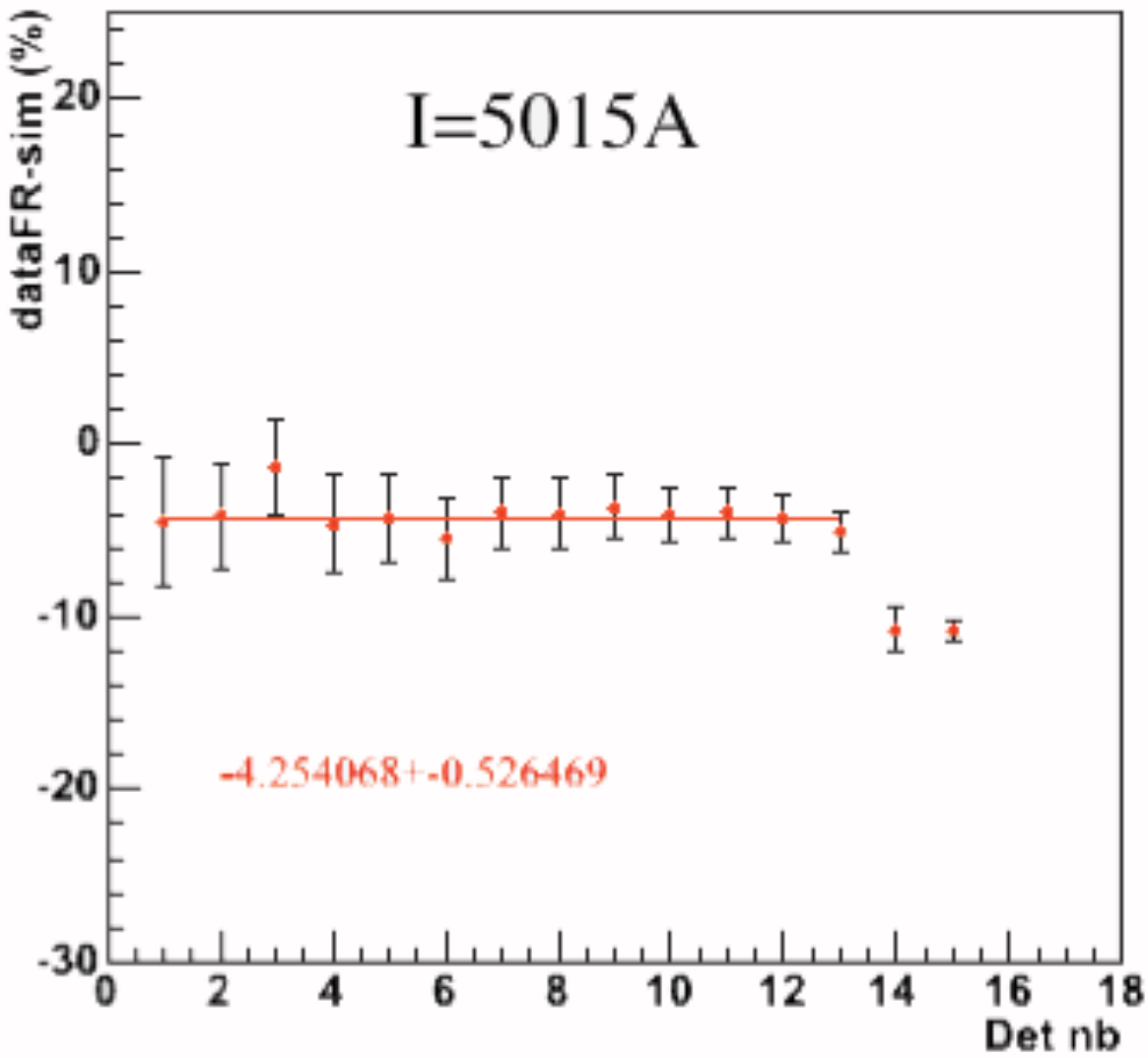

Figure 3.4: Comparison of detector rates between simulation and data for magnet current at 5015 A. Good agreement is seen between the two, with maximum excursion in detectors 14 and 15 . These excursions originate both from the difficulty in simulating the inelastic proton rates as well as from the fact that detectors 14 and 15 are sensitive to a relatively wide range of $Q^{2}$.

Runs were taken down to 4700 A, and Fig. 3.5 shows the elastic proton rate in detectors 15 and 16 as a function of the magnet current. The fit shows that the SMS current at the nominal $5000 \mathrm{~A}$ runs is closer to $4985 \pm 10 \mathrm{~A}$.

The second method is to simulate the tof separation between the elastic protons and pions, and compare to data. By adjusting the magnetic field in the simulation, the separation between the two peaks can be varied. In addition to the magnetic field strength, this method also accounts for uncertainties coming from misalignments in the detectors. Specifically, the longitudinal and radial offsets of the detectors are included in accounting for the pion-proton tof difference. For each 
Detector 15

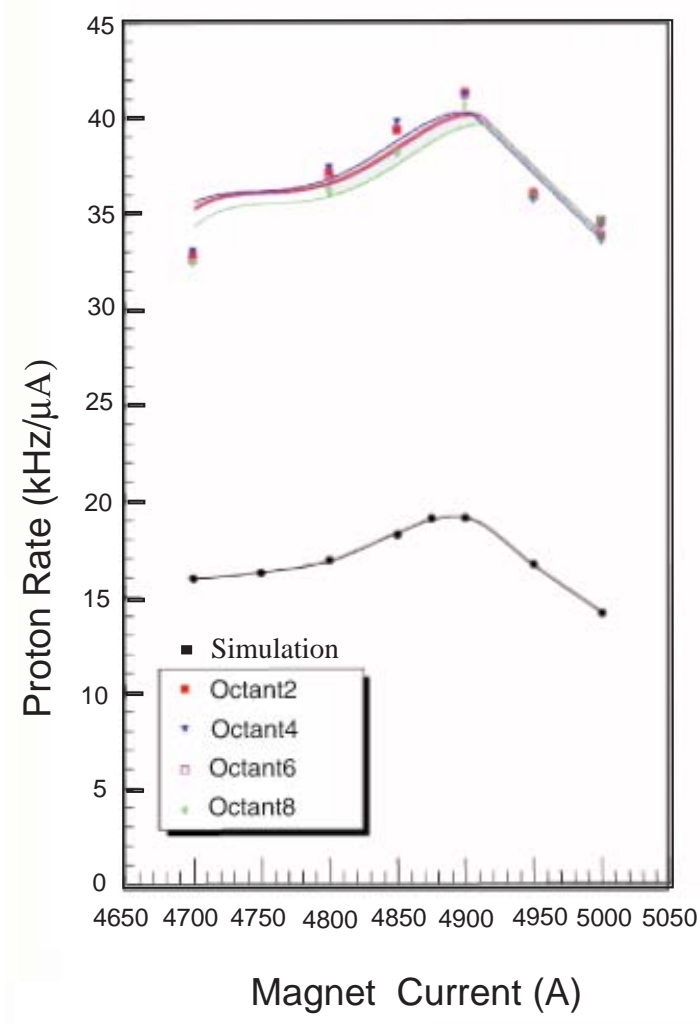

Detector 16

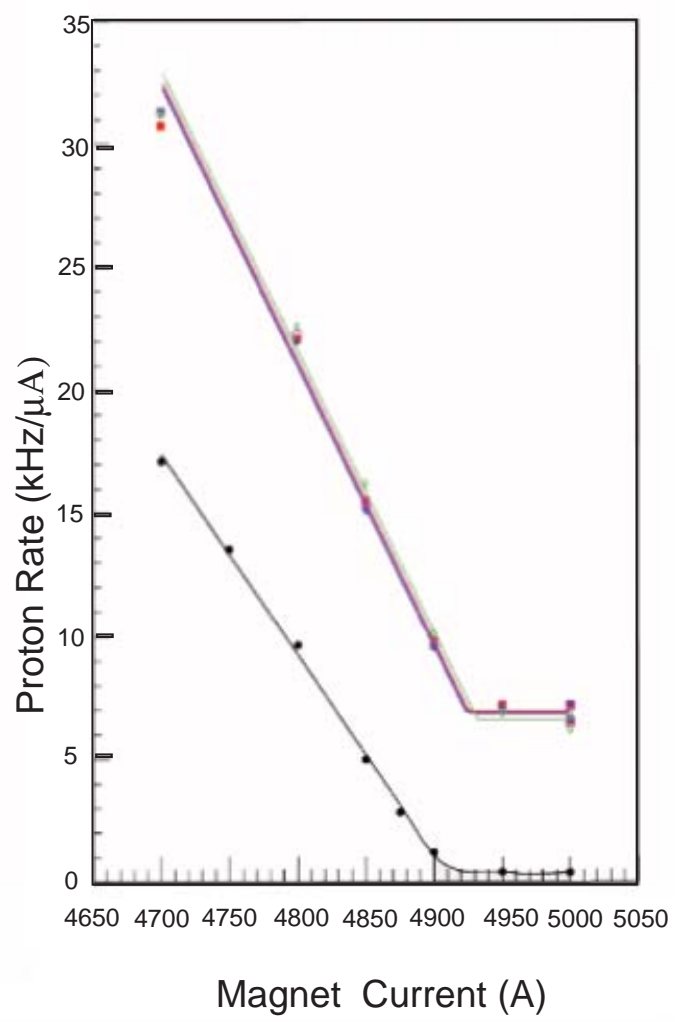

Figure 3.5: Detectors 15 and 16 elastic proton rate vs magnet current. Below 4900 A, the elastic protons can be seen hitting detector 16 .

detector, this tof separation, to first order, can be written as

$$
\Delta t=\Delta t_{\text {nominal }}+a_{\Delta t}^{B}\left(\frac{B}{B_{\text {nominal }}}-1\right)+a_{\Delta t}^{X} \Delta X+a_{\Delta t}^{Z} \Delta Z
$$

where $\Delta t_{\text {nominal }}$ and $B_{\text {nominal }}$ are the tof separation and magnetic field at $5000 \mathrm{~A}, \Delta t$ is the measured time separation, $B$ is the true field value, $\Delta X$ and $\Delta Z$ are the radial and longitudinal positions of the detectors, and $a_{\Delta t}^{B}, a_{\Delta t}^{X}$, and $a_{\Delta t}^{Z}$ are coefficients associated with the expansion. Similarly, the uncertainty in the $Q^{2}$ can be expanded as

$$
\frac{\Delta Q^{2}}{Q_{\text {nominal }}^{2}}=a_{Q^{2}}^{B}\left(\frac{B}{B_{\text {nominal }}}-1\right)+a_{Q^{2}}^{X} \Delta X+a_{Q^{2}}^{Z} \Delta Z
$$


where $\Delta Q^{2}$ is the uncertainty in the $Q^{2}$, and $Q_{\text {nominal }}^{2}$ is the $Q^{2}$ of any particular detector at 5000 A. Constraints are imposed in order to reduce the number of unknowns such that the system of linear equations can be solved. These constraints include a common $\Delta Z$ for all detectors, and that the longitudinal and radial shifts average to zero.

Shown in Fig. 3.6 is a comparison between simulation and data at a magnet current of $5000 \mathrm{~A}$. The position offsets, up to $1 \mathrm{~cm}$ radially and $1.6 \mathrm{~cm}$ longitudinally, determined from this method are too large compared to what can be expected from the mechanical structure of the ferris wheel frame. It is possible that the first order expansion in the offsets, or the constraints imposed on these offsets are incorrect or insufficient to decouple their contributions from the magnetic field strength. Therefore, what we find in the end is the overall average magnet current, and the total uncertainty from the magnetic field and any mis-alignments of the detectors. After fitting the magnet currents derived from each detector, we find an average magnet current of 5015 A with a variation of about $20 \mathrm{~A}$ around this central value. This is the value used for the determination of the $Q^{2}$ due to the fact that the method accounts for additional factors other than the magnetic field strength.

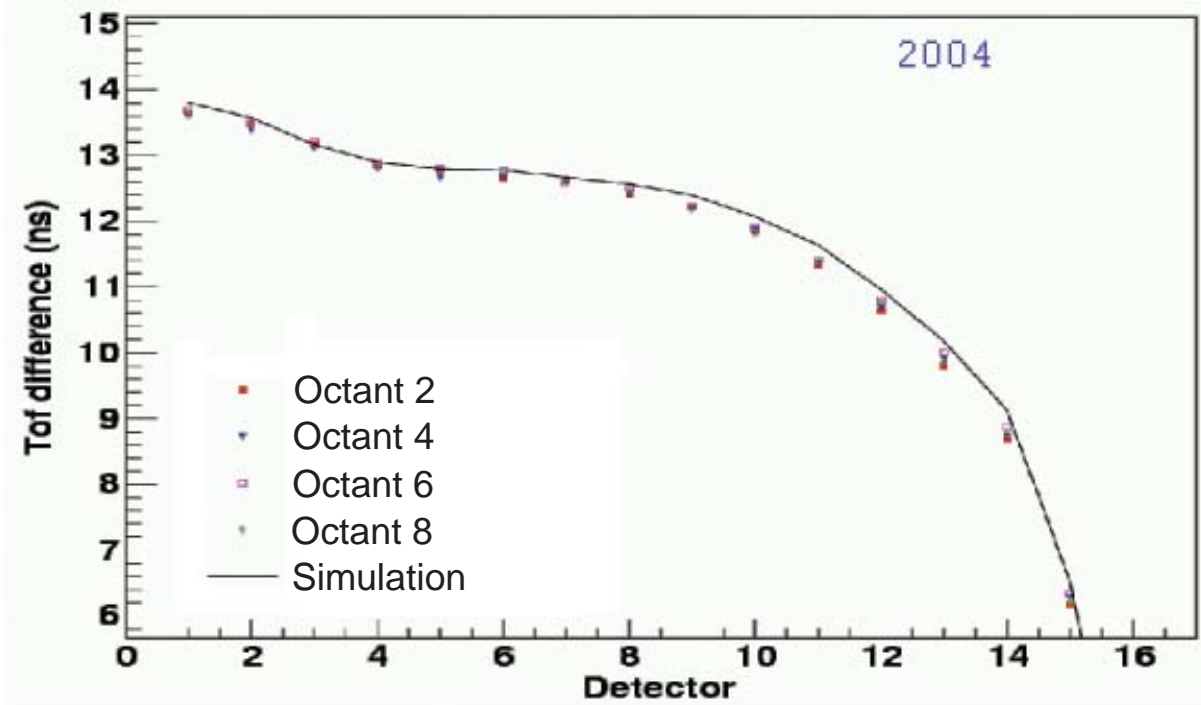

Figure 3.6: Comparison of pion-elastic proton tof separation as a function of detector. The black line indicates simulation, whereas the points represent data. This simulation is performed at a magnet current of $5000 \mathrm{~A}$.

From the simulation at $5015 \mathrm{~A}$, a $Q^{2}(t)$ spectrum for each detector is then determined, and integrated over the elastic proton peak weighing by the yield (similar to what is done in Eqn. 3.6 
for the asymmetry)

$$
<Q^{2}>_{\operatorname{det}}=\frac{\int Q^{2}(t) Y_{\text {meas }}(t)}{\int Y_{\text {meas }}(t)}
$$

where $<Q^{2}>_{\text {det }}$ is the central value of $Q^{2}$ for the elastic peak for a particular detector. The resulting $Q^{2}$ for each detector can be seen in Table 3.7 together with the parity-violating physics asymmetry.

The rest of this chapter will detail what is involved in the corrections applied to the raw proton asymmetry in order to determine the final parity-violating asymmetry for the $G^{0}$ forward angle experiment.

\subsection{Leakage correction}

As discussed in the experimental apparatus section, the JLab accelerator provides beam to all three halls simultaneously. This is accomplished by alternating beam pulse delivery to the three halls at $499 \mathrm{MHz}$. The beam for each hall is generated by a separate laser; each must turn off after a beam pulse such that each beam do not interfere with delivery to the other halls. However, since the lasers take a finite amount of time to turn off, some of the laser light leaks into the beam pulses of the other halls. Furthermore, each laser has a DC component due to their amplified spontaneous emission (ASE), which is not modulated at either $31 \mathrm{MHz}$ or $499 \mathrm{MHz}$, thus creating DC electron beams for all halls. Although these are common problems for all three halls, they are particularly problematic for the $G^{0}$ experiment due to its unique $31 \mathrm{MHz}$ beam structure and tof separation technique. The pulse frequency for $G^{0}$ at $31 \mathrm{MHz}$ is $\frac{1}{16}$ of the usual $499 \mathrm{MHz}$ JLab beam frequency. Therefore, for $G^{0}$ there should be no beam for 15 of the 16 beam buckets. A $499 \mathrm{MHz}$ leakage current (2 ns pulses) as well as a DC current would violate this requirement, and would appear as a global background underneath the $32 \mathrm{~ns}$ tof spectrum. The leakage contamination from the Halls A and B beams to the $G^{0}$ beam is about $50 \mathrm{nA}$ out of the nominal $40 \mu \mathrm{A}$.

If the only contribution is a dilution of the $G^{0}$ beam, then the false asymmetry would simply be proportional to the ratio between the $G^{0}$ and leakage beam currents. Depending on the $Q^{2}$, this would be a false asymmetry of order 1 to $10 \mathrm{ppb}$. Such a false asymmetry would be insignificant compared to the desired overall uncertainty. However, measurements of the properties of the leakage 
beam show large charge asymmetry and position differences. The parity quality of the leakage beam is poor due to the fact that the $G^{0}$ feedback scheme only corrects the $G^{0}$ beam. The IA (Intensity Asymmetry) cell and the PZT (Piezo-electric transducer) mirror can only affect the $G^{0}$ laser, but not the beam from the other two halls.

Therefore, in order to determine the contribution of the leakage current to the measured asymmetry, we consider both the size of the leakage as well as its charge asymmetry. The measured yield can be written in terms of contributions coming from the leakage and the main $G^{0}$ beam currents

$$
Y_{\text {meas }}=\frac{Y_{G^{0}} I_{G^{0}}+Y_{L} I_{L}}{I_{G^{0}}+I_{L}}
$$

where $Y_{G^{0}}$ and $Y_{L}$ are the yields from the $G^{0}$ and leakage currents, respectively, and $Y_{\text {meas }}$ is the measured yield. The asymmetry is

$$
A_{\text {meas }}=\frac{Y_{\text {meas }}^{+}-Y_{\text {meas }}^{-}}{Y_{\text {meas }}^{+}+Y_{\text {meas }}^{-}}
$$

so combining this with Eqn. 3.11, the measured asymmetry, $A_{\text {meas }}$, can be written in terms of the leakage and main $G^{0}$ beam contributions

$$
A_{\text {meas }}=\frac{R_{G^{0}}}{R_{\text {meas }}} A_{Y_{G^{0}}}+\frac{R_{L}}{R_{\text {meas }}} A_{Y_{L}}+\left(\frac{R_{G^{0}}}{R_{\text {meas }}}-\frac{I_{G^{0}}}{I_{\text {meas }}}\right) A_{I_{G^{0}}}+\left(\frac{R_{L}}{R_{\text {meas }}}-\frac{I_{L}}{I_{\text {meas }}}\right) A_{I_{L}}
$$

where $R_{G^{0}}$ and $R_{L}$ are the $G^{0}$ and leakage rates, respectively, $A_{Y_{G^{0}}}$ and $A_{Y_{L}}$ are the asymmetries of the $G^{0}$ and leakage rates as seen in the FPDs, and $A_{I_{G^{0}}}$ and $A_{I_{L}}$ are the charge asymmetries associated with the $G^{0}$ and leakage beams. At this point, a couple of approximations can be made. The charge asymmetry measured by the BCMs is a weighted average of $A_{I_{G^{0}}}$ and $A_{I_{L}}$

$$
A_{I_{\text {meas }}}=\frac{I_{G^{0}}}{I_{\text {meas }}} A_{I_{G^{0}}}+\frac{I_{L}}{I_{\text {meas }}} A_{I_{L}}
$$

Combining Eqn. 3.14 with the relation for the measured rate (similar to Eqn. 3.11), Eqn. 3.13 can 
be re-written as

$$
A_{\text {meas }}=\frac{R_{G^{0}}}{R_{\text {meas }}} A_{Y_{G^{0}}}+\frac{R_{L}}{R_{\text {meas }}} A_{Y_{L}}+\left(\frac{R_{G^{0}}}{R_{\text {meas }}} \frac{I_{\text {meas }}}{I_{G^{0}}}-1\right) A_{I_{G^{0}}}-\frac{\frac{I_{L}}{I_{G^{0}}}-\frac{R_{L}}{R_{G^{0}}}}{1+\frac{R_{L}}{R_{G^{0}}}} A_{I_{L}}
$$

Furthermore, since the leakage current, and hence the detector rate, is three orders of magnitude smaller than that from the main $G^{0}$ beam current, $R_{G^{0}} \approx R_{\text {meas }}, R_{L}<<R_{G^{0}}$, and $R_{L}<<R_{\text {meas }}$. Therefore, $\frac{R_{G^{0}}}{R_{\text {meas }}} \frac{I_{\text {meas }}}{I_{G^{0}}}-1 \approx 0$, and $\frac{R_{L}}{R_{\text {meas }}} \approx 0$. So, Eqn. 3.13 can be further simplified to

$$
A_{\text {meas }} \approx A_{Y_{G^{0}}}-\frac{I_{L}}{I_{G^{0}}}\left(1-\frac{Y_{L}}{Y_{G^{0}}}\right) A_{I_{L}}
$$

The false asymmetry caused by the charge asymmetry of the leakage beam can thus be written in terms of the leakage $\left(I_{L}\right)$ and $G^{0}\left(I_{G^{0}}\right)$ beam currents

$$
A_{\text {false }}^{\text {charge }}=\frac{I_{L}}{I_{G^{0}}}\left(1-\frac{Y_{L}}{Y_{G^{0}}}\right) A_{I_{L}}
$$

where $A_{\text {false }}^{\text {charge }}$ is the false asymmetry caused by the leakage charge asymmetry.

\section{The Cuto Technique}

The measurement of helicity-correlation in the leakage beam is not straightforward. Since the $G^{0}$ DAQ monitors the sum of the $G^{0}$ and leakage currents, monitoring the BCMs and BPMs would not give the parity quality of the pure leakage beam. Turning off the Hall $\mathrm{C}$ laser to measure the pure Hall A and B leakage is not possible with BCMs, as $50 \mathrm{nA}$ is less than the precision of these monitors. The leakage charge asymmetry is therefore determined by the luminosity monitors by measuring the asymmetry in its rates. Such dedicated leakage runs are not, however, sufficient to determine the leakage beam quality for the entire forward angle measurement. In order to get a run-by-run assessment of the leakage beam quality, the asymmetry measured in the region of the tof spectrum dominated by the leakage current is used.

The tof spectrum due to the leakage compared to the nominal $40 \mu \mathrm{A}$ data can be seen in Fig. 3.7. There are typically six cuts monitored in a tof spectrum. Two of these are the particle cuts defined by the pion and elastic peaks. Cuts 1 through 3 shown in Fig. 3.7 monitor parts of the tof spectrum 


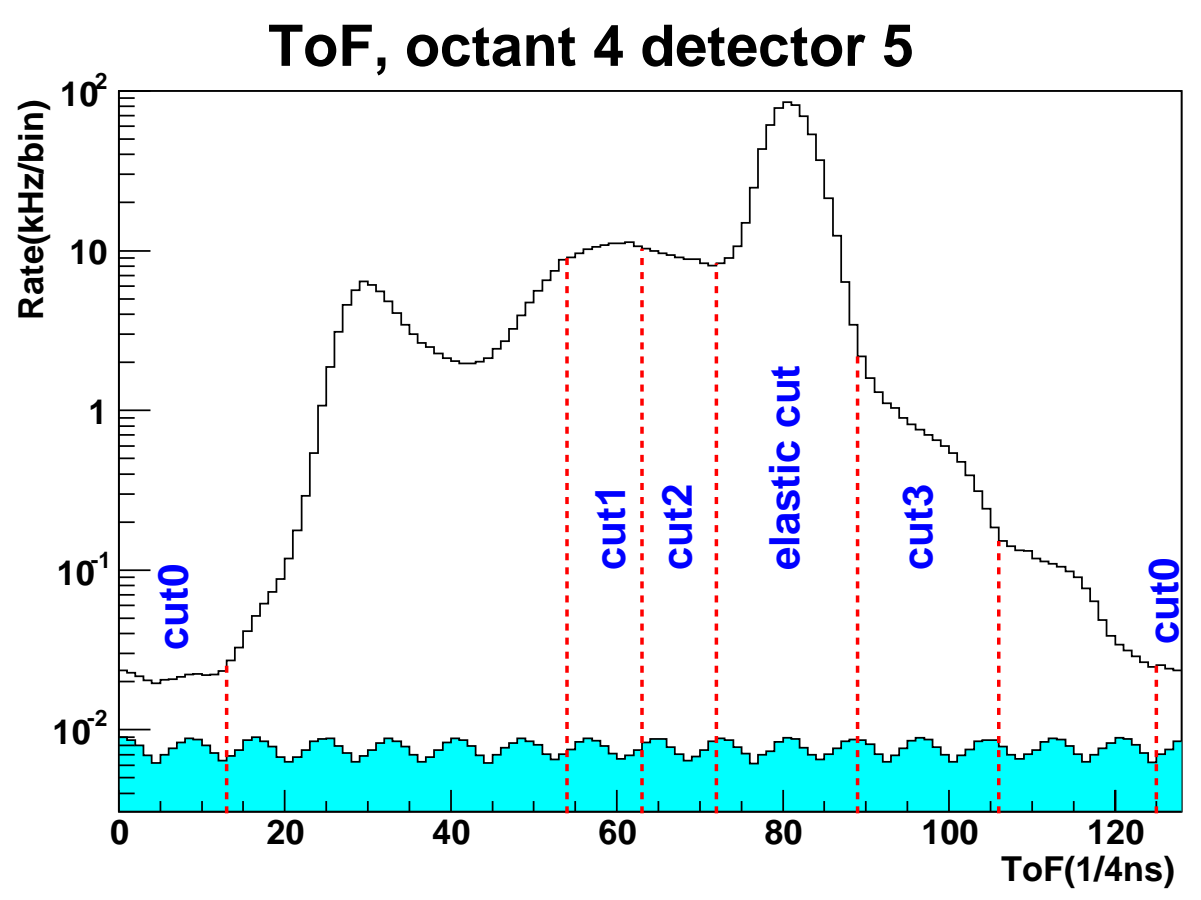

Figure 3.7: Leakage rates (blue) compared to the overall $G^{0}$ rates (black). The leakage rates are actual measured rates. The shape of the leakage spectrum originates from the time structure of the leakage current. Each 2 ns leakage pulse creates its own tof spectrum, shifted 2 ns with respect to the previous 2 ns pulse. The sum of all 16 leakage pulses creates a modulated shape seen here.

with differing contributions from the inelastically scattered protons. The region defined as cut0 is the part of the spectrum consisting of rates from the leakage beam and background rates from the main $G^{0}$ beam, and is crucial in measuring the effects of the leakage current. Typical values associated with the leakage and $G^{0}$ beams can be found in Table 3.1.

\begin{tabular}{|c|c|c|c|c|c|}
\hline Cuts & $R_{\text {meas }}(k H z)$ & $R_{L}(k H z)$ & $R_{G^{0}}(k H z)$ & $Y_{G^{0}}(k H z / \mu A)$ & $Y_{L}(k H z \mu A)$ \\
\hline Elastic Protons & 647.1 & 0.2054 & 646.9 & 16.19 & 4.11 \\
\hline Cut0 & 5.97 & 2.47 & 3.5 & 0.09 & 49.4 \\
\hline
\end{tabular}

Table 3.1: Rates and yields observed due to leakage and $G^{0}$ beams for the elastic cut and cut0.

From cut0, we wish to deduce two values; the leakage current, and the leakage asymmetry. This allows us to monitor these values on a run-by-run basis through cut0. The rate measured in cut0 can be written as the sum of the leakage and $G^{0}$ background components as

$$
R_{\text {meas }, c u t 0}=R_{L, c u t 0}+R_{G^{0}, b k g}
$$


where $R_{\text {meas,cut } 0}$ and $R_{L, c u t 0}$ are the measured and leakage rates in cut0, and $R_{G^{0}, b k g}$ is the background rate from the main $G^{0}$ beam. These contributions can be empirically determined by turning off the $G^{0}$ laser, and observing the rates coming from the Hall A and B lasers. Furthermore, the contribution from the DC component (coming from the ASE) can be determined by passing the $G^{0}$ beam through the Hall A chopper slit and observing the "leakage" into Hall C. From these measurements, the total $G^{0}$ background rate of $3.5 \pm 0.17 \mathrm{kHz} G^{0}$ is determined in cut0 according to Eqn. 3.18.

Once $R_{G^{0}, b k g}$ is determined, the leakage rate can be deduced on a run-by-run basis in cut0. The leakage current can in turn be deduced as

$$
I_{L, \text { deduced }}=\frac{\frac{R_{L, c u t 0}}{w_{\text {cut } 0}}}{Y_{G^{0}}}=\frac{\frac{R_{\text {meas }, \text { cut } 0}-3.5 k \mathrm{~Hz}}{w_{c u t} 0}}{Y_{G^{0}}}
$$

where $w_{\text {cut } 0}$ is the total width of cuto, and $Y_{G^{0}}$ is the tof-averaged $G^{0}$ yield.

The charge asymmetry of the leakage beam in cut0 can be related to the asymmetry of the rates $[84]$

$$
\begin{gathered}
A_{I_{L}}=\frac{R_{\text {meas }, \text { cut } 0}}{R_{L, c u t 0}} A_{\text {cut } 0}=\frac{R_{\text {meas }, \text { cut } 0}}{R_{\text {meas }, \text { cut } 0}-3.5 k H z} A_{\text {cut } 0} \\
\sigma_{A_{I_{L}}}=\frac{R_{\text {meas }, \text { cut } 0}}{R_{\text {meas }, \text { cut } 0}} R_{\text {meas }, \text { cut } 0}-3.5 k H z \sigma_{A_{\text {cut } 0}}
\end{gathered}
$$

where $A_{c u t 0}$ is the measured cut0 asymmetry, and $A_{I_{L}}$ is the leakage charge asymmetry.

\section{Cuto Certification}

The verification of the cut0 technique involves comparing the leakage current and asymmetry values deduced through Eqn. 3.19 and 3.20 with those measured by turning off the Hall C laser and directly observing the leakage beam. Fig. 3.8 shows this comparison through a correlation plot. We can see that the correlation is near $1: 1$.

Fig. 3.9 shows the leakage current and charge asymmetry during the forward angle production run as deduced from the cut0 yields and asymmetries. The resulting parity quality of the leakage beam shows a charge asymmetry of about $350 \mathrm{ppm}$.

In order to determine the systematic uncertainty from the leakage correction, we note from 

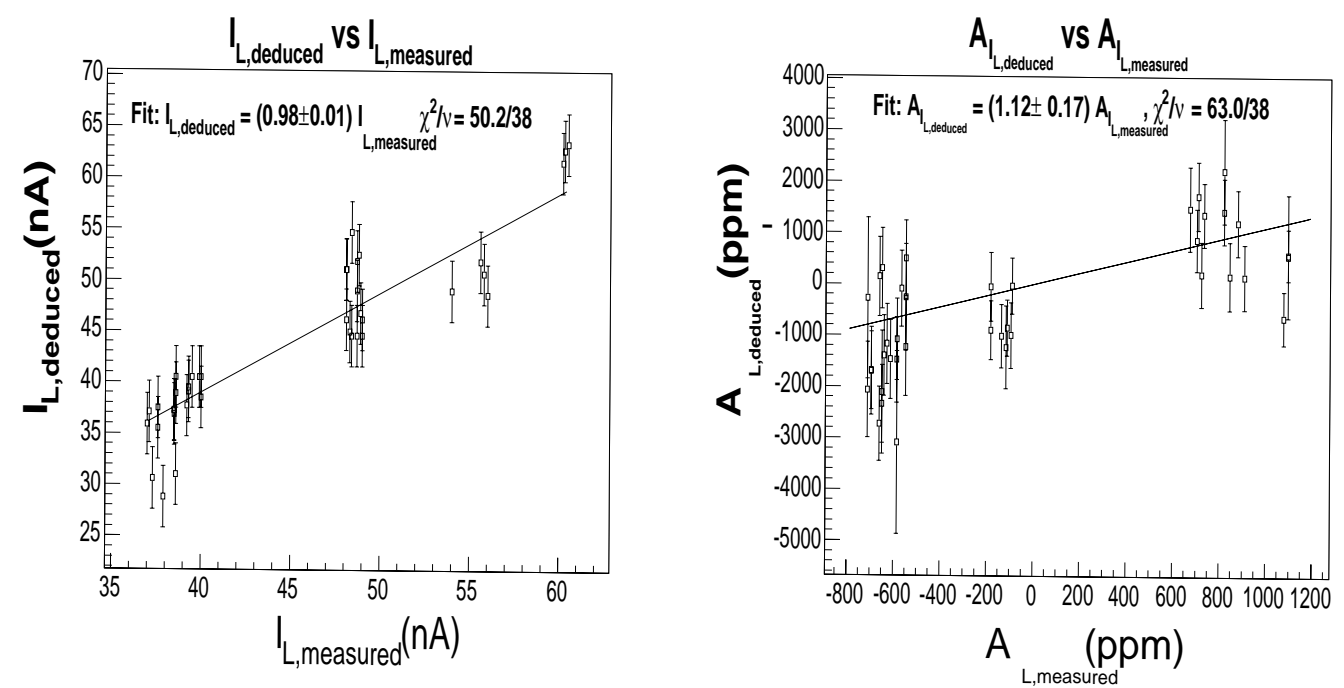

Figure 3.8: Comparison of deduced and measured leakage current and asymmetry. The two values agree well over a range of beam currents and charge asymmetries.

Eqn. 3.16 that the correction itself is additive where Eqn. 3.17 shows the false asymmetry. Therefore, if one were to plot the measured asymmetry, $A_{\text {meas }}$, against the false asymmetry, the slope should be -1 in the case the correction is perfect. A slope more negative than -1 means that we have under-corrected, while a less negative slope would imply over-correction. Fig. 3.10 shows such a plot for the elastic proton cut and cut3. A linear fit on the elastic proton cut agrees well with -1, while that from cut3 agrees to within $20 \%$, but may suggest some under-correction.

In addition, an empirical approach has also been taken to determine the uncertainty in the leakage correction. The leakage correction scheme is applied for data taken at different beam currents (at 10, 20, and $40 \mu \mathrm{A}$ ). We make the assumption that at $40 \mu \mathrm{A}$, the corrected asymmetry approaches the physics asymmetry (since the leakage contribution is fractionally lower than for lower beam currents). Then, for lower beam currents, the false asymmetry calculated from Eqn. 3.17 can be compared to what correction is needed to bring the measured asymmetry to the physics asymmetry. The two calculations agree to $\sim<20 \%$ in all cases. We therefore assign an uncertainty of $20 \%$ to the false asymmetry from the leakage correction.

In the end, we wish to know the uncertainty and false asymmetry contribution to the elastic physics asymmetry, $A_{e l}$. In order to obtain $A_{e l}$, we must make an additional background correction to remove the contamination from the inelastic protons from underneath the elastic peak. Details of 

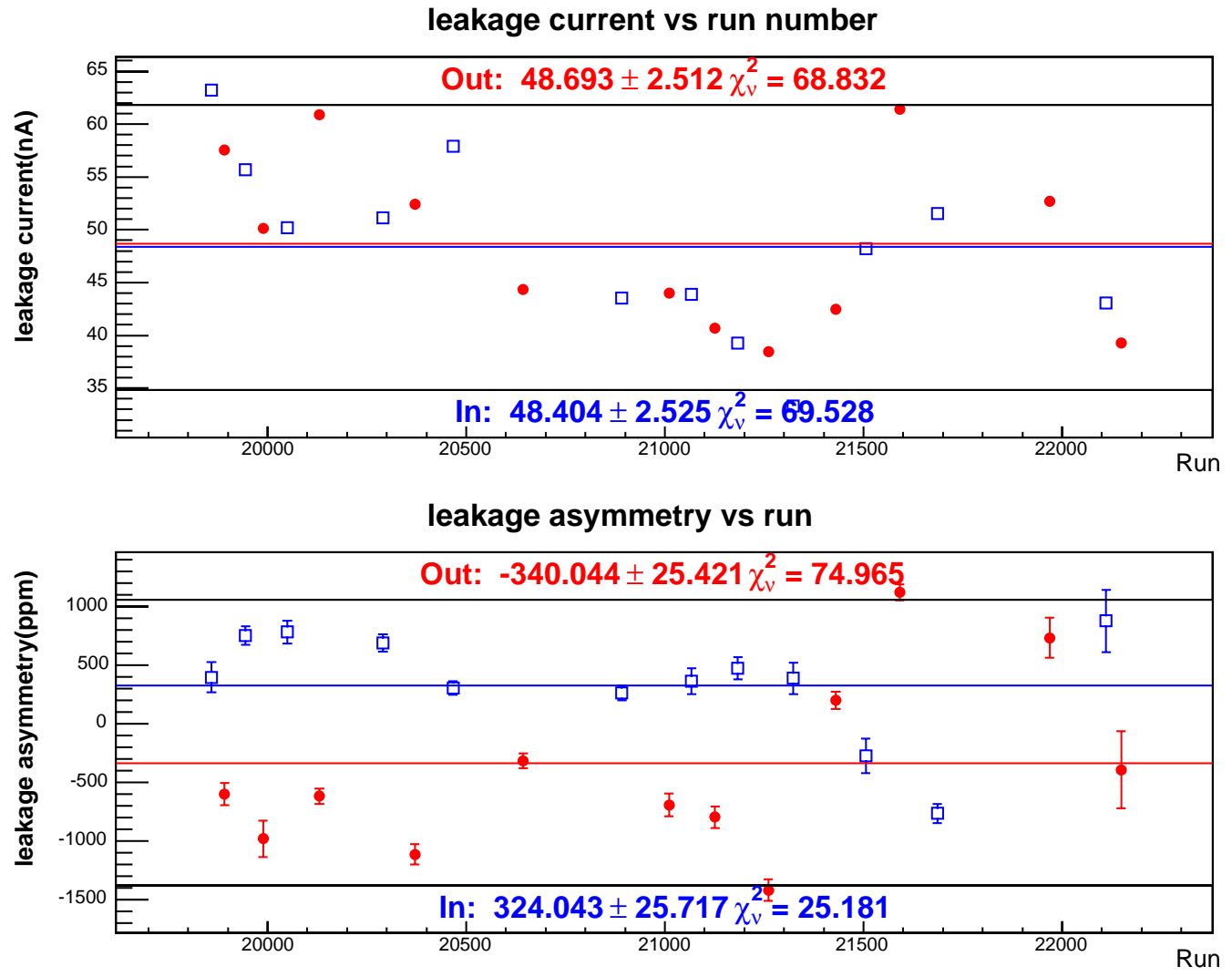

Figure 3.9: Leakage current (top) and charge asymmetry (bottom) for the forward angle production run. Each point represents the integrated charge asymmetry and average current over several weeks.

the background correction scheme can be found in Section 3.8. For the purpose of the leakage correction, we apply this background correction to data that are corrected $\left(A_{e l, c o r r}\right)$ and un-corrected $\left(A_{e l, r a w}\right)$ for the leakage. The difference between the two values is the leakage contribution to the elastic asymmetry. The false asymmetry from the leakage charge asymmetry is $0.71 \pm 0.14 \mathrm{ppm}$.

The false asymmetry from the leakage position differences is similar, except the sensitivity of the detectors to changes in beam position must be known

$$
A_{\text {false }}^{\text {position }}=\frac{I_{L}}{I_{G^{0}}} \frac{d Y}{d P} \delta P_{\text {leak }}
$$

where $\delta P_{\text {leak }}$ is the leakage position difference, and $\frac{d Y}{d P}$ is the sensitivity of the $G^{0}$ spectrometer to the leakage beam position. This sensitivity slope of the $G^{0}$ spectrometer to beam position is safely estimated to be the same for that of the standard $G^{0}$ beam, or about $0.1 \% / \mathrm{mm}$ averaged over all 

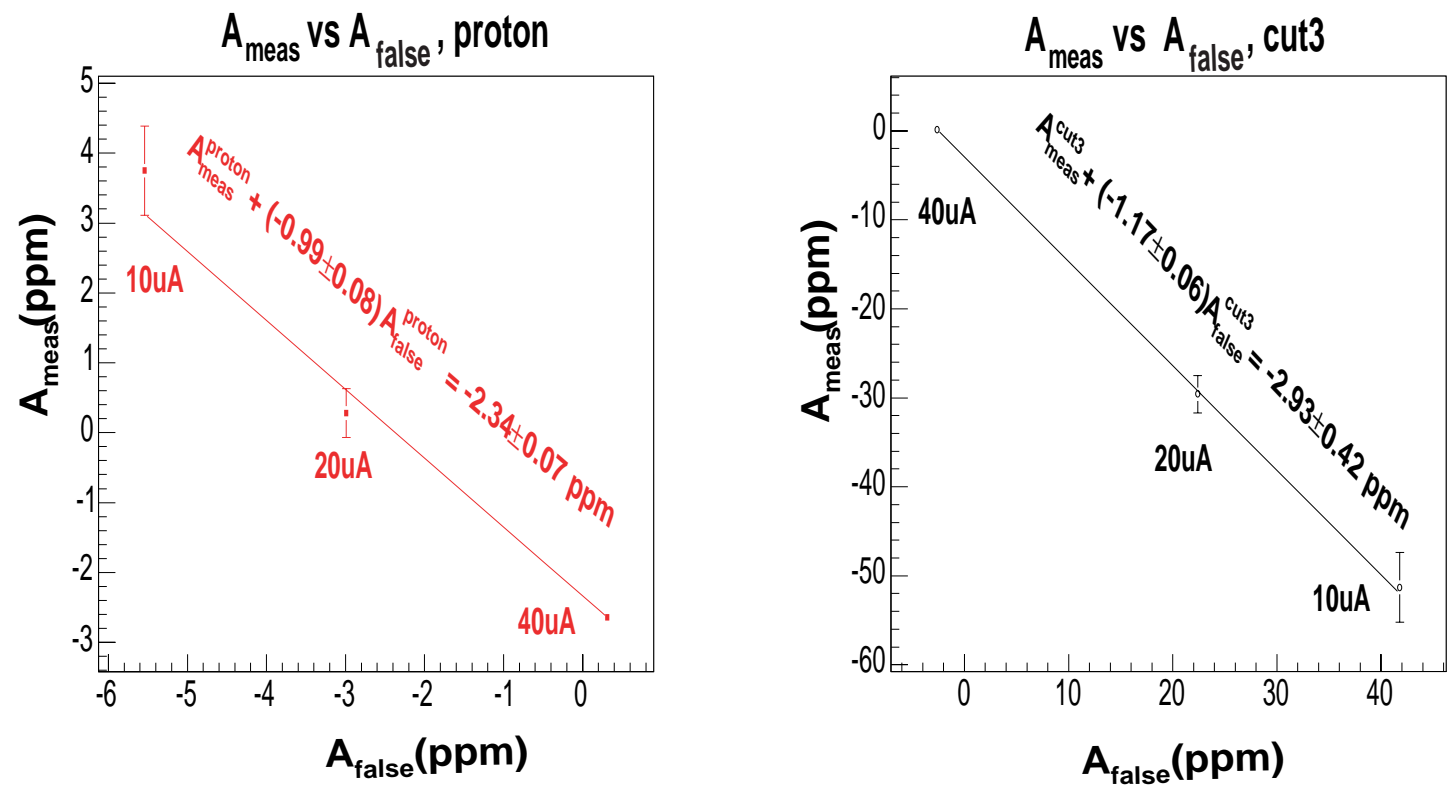

Figure 3.10: Correlation plots for $A_{\text {meas }}$ and $A_{\text {false }}$ for the elastic proton cut and cut3. The elastic slope is -1 as expected, while that of cut3 agrees with -1 to within $20 \%$.

octants, given the size of the potential correction and the fact that the sensitivity is a characteristic of the spectrometer which is independent of the source of the beam. Fig. 3.11 shows the position differences of the leakage current as a function of run-number. The leakage position differences are seen to be 100's of nm, which is two orders of magnitude larger than what is seen for the main $G^{0}$ beam. However, even if one were to assume a leakage position difference of $1 \mu m$, the false asymmetry comes out to be of order $1 \mathrm{ppb}$, which is much less than the precision of the physics asymmetry measurements in $G^{0}$, and thus can be safely neglected.

\subsection{Deadtime correction}

Deadtime corrections are an inherent systematic in parity-violation counting experiments such as $G^{0}$. The $G^{0}$ electronics counts individual protons detected by the FPDs at a rate of about $1 \mathrm{MHz}$. However the electronics for any given FPD segment requires a finite amount of time to process the detection of a particle. During this time (deadtime), the electronics are effectively blind to any particle that may strike the FPD. Since any proton hitting the FPD during this time is not recorded, deadtime has the effect of altering the counting rate and ultimately the measured parity-violating 


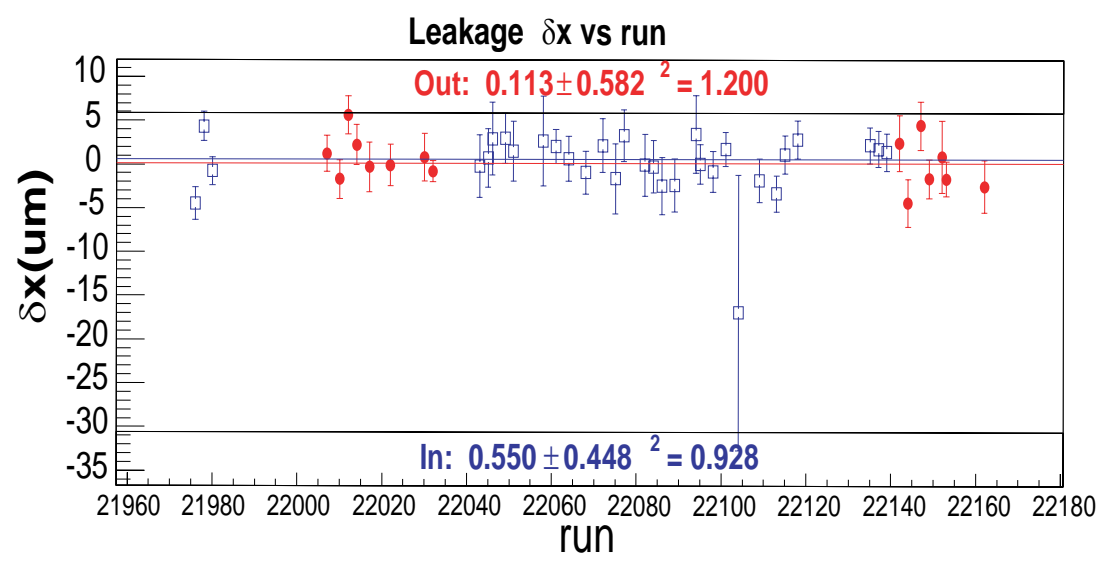

Leakage $\delta$ y v run

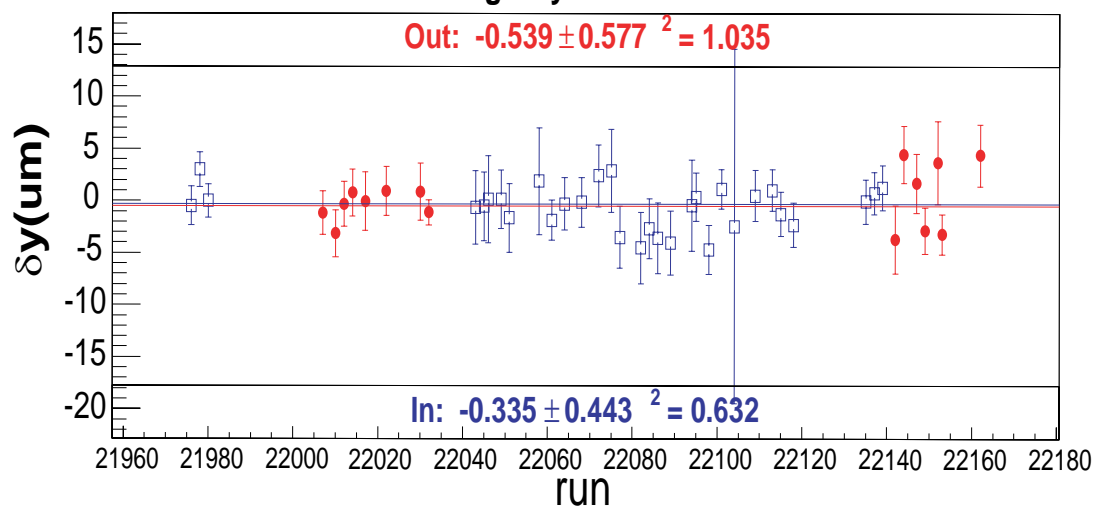

Figure 3.11: Leakage position differences vs run-number. The position differences are large but within $1 \mu \mathrm{m}$.

asymmetry.

The effect of deadtime is addressed in part by a next pulse neutralization (NPN) scheme. In this scheme, if an event produced by beam pulse $\mathrm{n}$ triggers the electronics, any data produced by the next beam pulse (32 ns later), $n+1$, for the same channel are ignored. This artificial deadtime of known length is included in both the NA and FR electronics. In addition to the NPN scheme, the global and differential buddy systems (see Section 2.5) serve to monitor helicity-correlation in the deadtime effects.

The probability or the deadtime fraction, $f_{D T}$, of any particular channel being dead is proportional to the event rate

$$
f_{D T}=\tau R_{\text {meas }}
$$

where $R_{\text {meas }}$ is the measured detector rate, $\tau$ is the deadtime, which in the NPN scheme is the 
time-length of a $32 \mathrm{~ns}$ beam pulse. Thus, to first order, the measured rate will be related to the true rate through the probability $f_{D T}$

$$
R_{\text {meas }}=\left(1-f_{D T}\right) R_{\text {true }}
$$

where $R_{\text {true }}$ is the true rate. The measured charge normalized yield, therefore, will be related

$$
Y_{\text {meas }}=\left(1-f_{D T}\right) Y_{\text {true }}
$$

to the true yield, $Y_{\text {true }}$, in the same manner. Since the rate is directly proportional to the beam current, any charge asymmetry in the beam can cause the deadtime effect to induce false asymmetries. The true asymmetry can be calculated from this deadtime fraction

$$
A_{\text {corr }}=\frac{A_{\text {meas }}}{1-f_{D T}}-f_{D T} A_{q}
$$

where $f_{D T}$ is the deadtime fraction, $A_{\text {meas }}$ is the measured asymmetry, $A_{q}$ is the charge asymmetry, and $A_{\text {corr }}$ is the asymmetry corrected for deadtime. As can be seen in Eqn. 3.26, the deadtime fraction, $f_{D T}$, is the correlation between the measured asymmetry, and the charge asymmetry.

The effect of deadtime on the measured asymmetry can be determined directly by measuring this correlation, and examining how they change once the corrections are applied. Once the correlation (or equivalently, the deadtime fraction) is determined, the measured asymmetry can be corrected according to Eqn. 3.26. An intensity asymmetry scan (see Section 2.2) artificially induces a large charge asymmetry to see how the measured asymmetry changes. Fig. 3.12 shows this correlation for each detector. The scans show a deadtime fraction of about $10 \%$ in the NA electronics, and $15 \%$ in the FR electronics, and a residual deadtime of about $1 \%$ is seen after the correction. With a charge asymmetry of $0.14 \mathrm{ppm}$ as shown in Table 2.2 , the false asymmetry from this residual deadtime is less than $0.01 \mathrm{ppm}$.

In order to determine the validity of the above method, a helicity-averaged test is conducted. The beam current is varied from 5 to $40 \mathrm{uA}$, and the variation of the measured yield due to deadtime is determined. This measured deadtime would be sufficient to correct the asymmetry; in addition, 


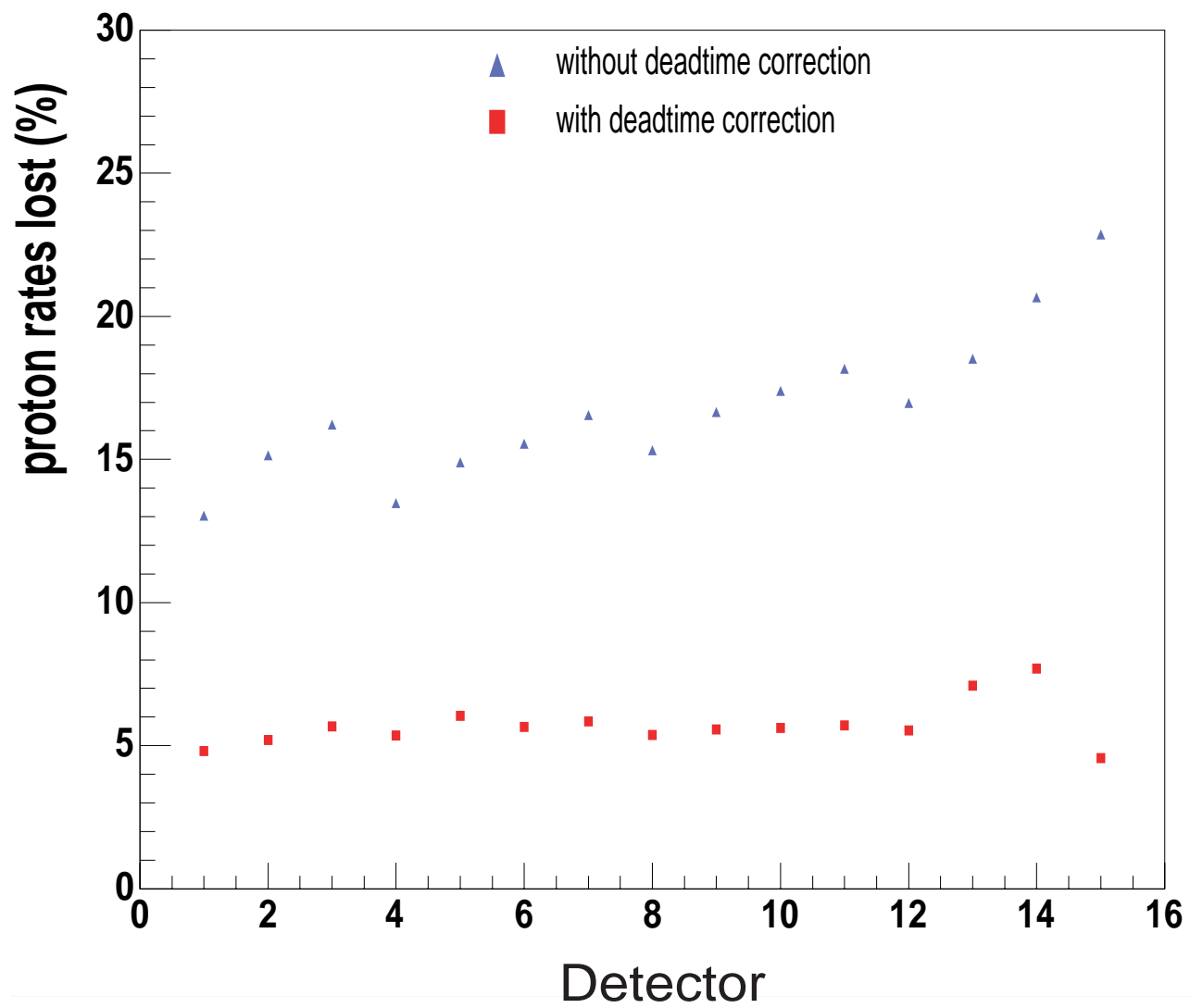

Figure 3.12: Correlation between the measured asymmetry and the charge asymmetry. The black points show the correlation before the correction, and the red points show the deadtime-corrected asymmetries. The derivatives shown here are the deadtime fraction, $f_{D T}$ seen in Eqn. 3.26.

we have modeled this deadtime using supplemental measurements to determine contributions to the overall effect from various stages of the electronics. The first correction applied is through the evaluation of the deadtime due to four-fold coincidence rates. The second correction does the same, except for "singles" rates, where 1, 2, or 3 PMTs detect an event. Since TEE's (time-encoding electronics) record all four-fold coincidence events, both NA and FR electronics use the TEE's for the first step, while the singles events are determined from the fastbus datastream. Fig. 3.13 shows the measured and corrected deadtime fraction for both the NA and FR electronics. The initial deadtime fraction is seen to be about 10 to $15 \%$ depending on the detector, consistent with what is seen in Fig. 3.12. The correction scheme is seen to reduce but undercorrect the yield, with the best correction giving a residual deadtime fraction of about $1 \%$, in agreement with the method of directly measuring the correlation. 


\section{$1 / \mathrm{YO} \mathrm{dY} / \mathrm{dl}{ }^{*} \mathbf{4 0} \%$}

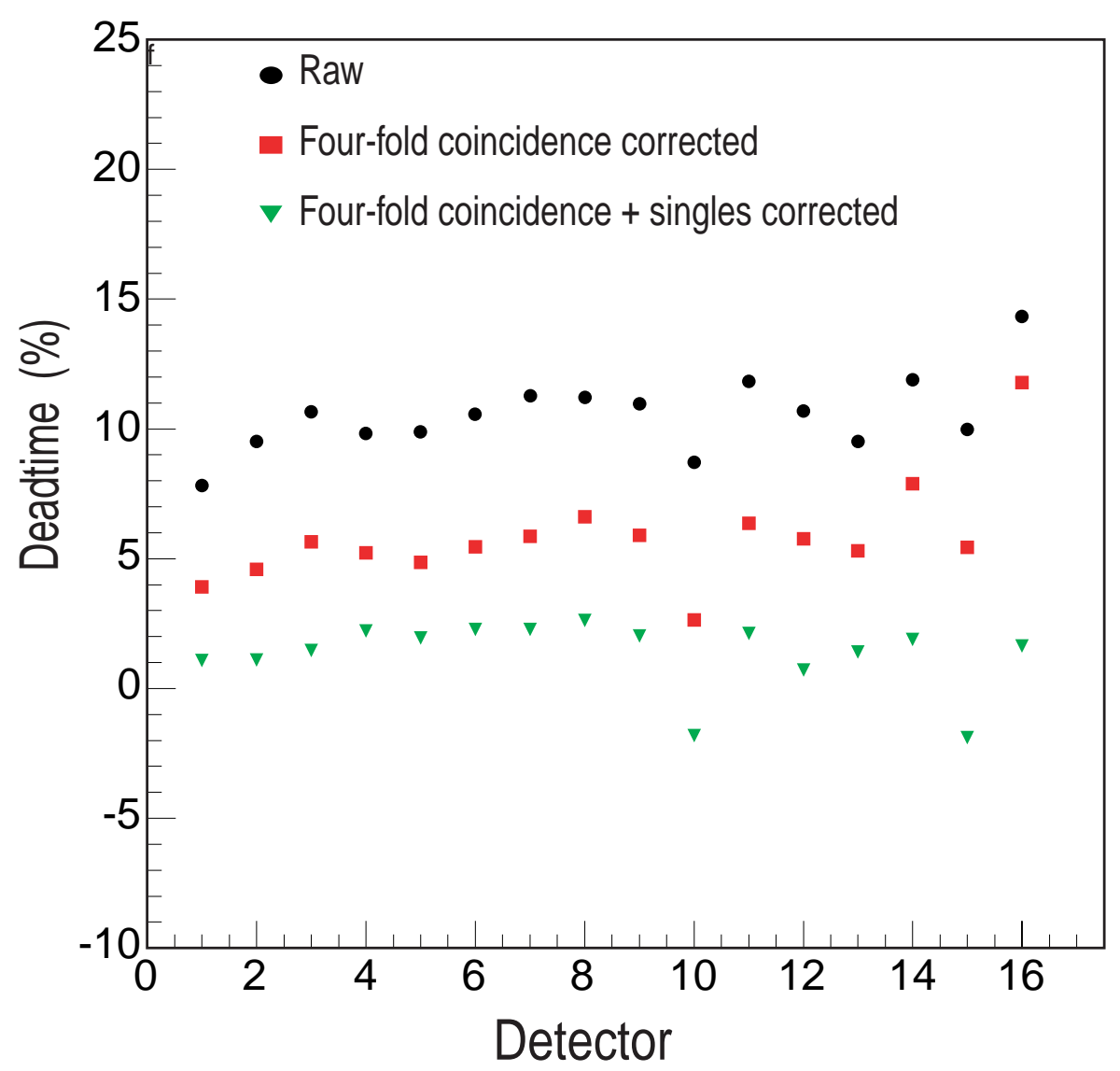

Figure 3.13: NA deadtime corrections. The uncorrected data (black) shows 10-15\% deadtime, while he four-fold and singles corrected data (green) shows residual deadtime of about $1 \%$.

\subsection{Linear Regression correction}

As mentioned in the beam section, helicity-correlated differences in various beam parameters can cause false asymmetries, as seen in Eqn. 2.14. It should be noted that although both the deadtime and linear regression corrections remove false asymmetry contributions from charge asymmetry, the two corrections are distinctly different. The effect of deadtime, as seen in Eqn. 3.26, is non-linear in $f_{D T}$ (and to the beam current) and is dependent on the tolerance of our electronics to high rates. However, the linear regression correction corrects effects where the spectrometer sensitivity couples to the helicity-correlation in the beam. Furthermore, because the helicity-correlated differences are 
very small, the response of the detector yield to these differences is assumed to be linear.

Therefore, the true yield in the detectors can be related to the measured yield as a linear combination of the beam parameters

$$
Y_{\text {meas }}=Y_{\text {corr }}+\Sigma_{i} \frac{d Y}{d P_{i}} P_{i}
$$

where $Y_{\text {corr }}$ is the true yield that is not contaminated by changes in beam parameters, $P_{i}$ represents the six beam parameters monitored for helicity-correlation, and $\frac{d Y}{d P_{i}}$ is the sensitivity of the detector yield to variations in beam parameter $P_{i}$. Forming an asymmetry using the relation in Eqn. 3.27, the asymmetry corrected for HC beam parameters can be related to the raw asymmetry. Eliminating factors of order $\Sigma_{i} \frac{d Y}{d P_{i}}$ from the denominator

$$
A_{\text {corr }}=A_{\text {meas }}+A_{\text {false }} \approx A_{\text {meas }}+\frac{1}{2<Y>} \Sigma_{i} \frac{d Y}{d P_{i}} \delta_{P_{i}}
$$

where $A_{\text {meas }}$ is the measured asymmetry, $\langle Y\rangle$ is the helicity-averaged yield, $A_{\text {false }}$ is the false asymmetry induced by the HC beam properties, $A_{\text {corr }}$ is the asymmetry corrected for such false asymmetries, and $\delta_{P_{i}}$ is the $\mathrm{HC}$ difference in the beam parameter. The overall $\mathrm{HC}$ differences in the beam parameters, averaged over the entire experiment, can be seen in Table 2.2 in the Section 2.2.

The slopes were calculated using three different methods. Two are based on measurements of the yield using natural beam motion (NBM) and coil pulsing (CP) as mentioned in the Section 2.2. The slopes calculated from NBM are used to determine the false asymmetry, while CP data are used to cross-check the NBM slopes. The position slopes are also calculated using a G0GEANT simulation.

The slopes are calculated from the data using standard multiple linear regression technique, taking into account the correlations between the six beam parameters and the yields as well as between the six beam parameters themselves

$$
\left(<\delta Y * d P_{i}>\right)=\left(<d P_{i} * d P_{j}>\right) \frac{d Y}{d P_{i}}
$$

where $<\delta Y * d P_{i}>$ is the vector representing the covariance between the yields and the beam 
parameters, while $<d P_{i} * d P_{j}>$ is a $6 \mathrm{x} 6$ matrix representing the correlation between the beam parameters. In the analysis we calculate the covariance terms involving parameter differences in terms of the parameter averages

$$
\begin{gathered}
<\delta Y * d P_{i}>=<Y * P_{i}>-<Y><P_{i}> \\
<d P_{i} * d P_{j}>=<P_{i} * P_{j}>-<P_{i}><P_{j}>
\end{gathered}
$$

for each run. Consequently, the slopes can be determined on a run-by-run basis. In order to fully implement the linear regression scheme, the data must be replayed twice. This is because the slopes cannot be determined until the mean values in the preceding equations are calculated first. Once the covariance terms are calculated, the slopes can be determined simply by inverting the 6x6 matrix in Eqn. 3.29.

The statistical uncertainty can be calculated in quadrature from the covariance terms

$$
\begin{aligned}
\sigma_{\frac{d Y}{d P_{i}}}^{2} & =<Y^{2}>-<Y>^{2} \\
& -2 * \Sigma_{j} \frac{d Y}{d P_{j}}\left(<Y * P_{j}>-<Y><P_{j}>\right) \\
& +\Sigma_{j} \Sigma_{k} \frac{d Y}{d P_{j}} \frac{d Y}{d P_{k}}\left(<P_{j} * P_{k}>-<P_{j}><P_{k}>\right)
\end{aligned}
$$

where $<Y^{2}>-<Y>^{2}$ and $<P_{j} * P_{k}>-<P_{j}><P_{k}>$ are the variance and covariance terms of the yield and the beam parameters, and $\left.\left\langle Y * P_{j}\right\rangle-<Y><P_{j}\right\rangle$ are the cross-terms. However, due to the high rate seen in the $G^{0}$ detectors, the statistical uncertainty in the slopes is five to six orders of magnitude smaller than the slopes themselves. The uncertainty in the slopes are therefore dominated by systematics that may come from instabilities in the beam as well as our apparatus. We therefore assign the standard deviation of the slopes over the entire production run as its uncertainty. A table of slopes for both insertable half waveplate states averaged over octants can be found in Appendix C.2.

After the slopes are calculated, the data are replayed for the second time to use the slopes to correct the yields. The primary motive of the second replay is to correct the asymmetry for HC beam properties. During the second replay, the corrections to the yields are made on an MPS-by- 

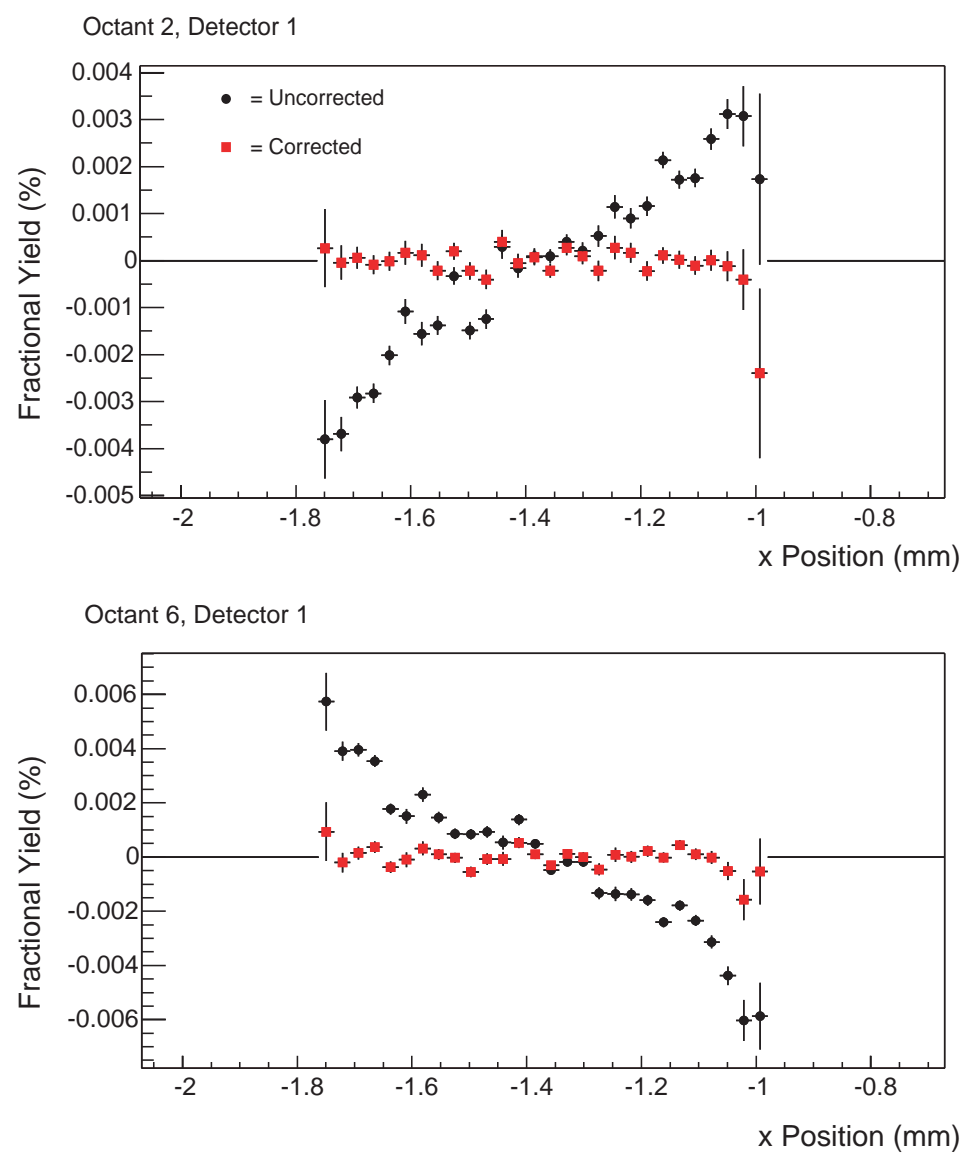

Figure 3.14: Fractional yield vs beam position. The detector's sensitivity to the beam position is corrected according to Eqn. 3.33. The corrected yield shows no sensitivity to beam fluctuations. Octants 2 and 6 , which are diametrically opposite octants, show sensitivities that are opposite in sign.

MPS basis independent of the helicity state (i.e. we assume the slopes are helicity independent)

$$
Y_{\text {corr }}^{M P S}=Y_{\text {meas }}^{M P S}-\Sigma_{i} \frac{d Y}{d P_{i}} * d P_{i}^{M P S}
$$

where $Y_{c o r r}^{M P S}$ and $Y_{m e a s}^{M P S}$ are the corrected and measured yields, respectively, of each MPS, $d P_{i}^{M P S}$ are the six beam parameters for the same MPS, and $\frac{d Y}{d P_{i}}$ are the six slopes calculated from the first replay. Fig. 3.14 shows an example of how the corrections procedure removes the beam position sensitivity of the yield for two particular detectors.

One of the characteristics of the yield distributions seen in Fig. 3.14 is that the uncorrected slopes have opposite signs depending on the octant. Since the spectrometer is azimuthally symmetric, 
diametrically opposite octants would have sensitivities to beam position and angle that are equal in magnitude but opposite in sign. This characteristic can be seen clearly when the slopes from the first replay are plotted against octant as in Fig. 3.15. The position and angle slopes show a
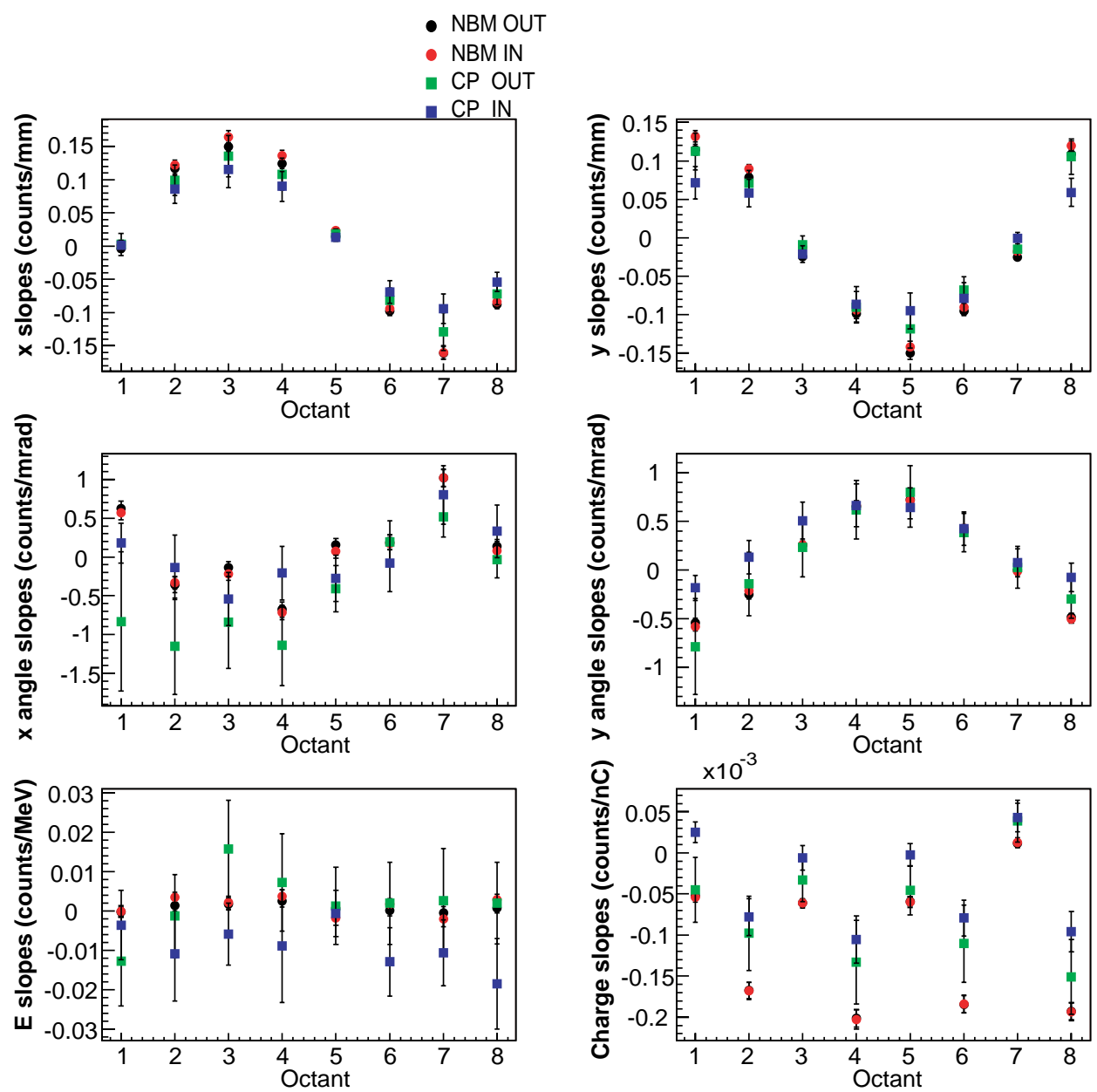

Figure 3.15: Linear regression slopes calculated from both NBM (Natural Beam Motion) and CP (Coil Pulsing) runs for both IHWP IN and OUT. Shown are slopes from detector 1.

clear octant dependence due to the azimuthally symmetric nature of the $G^{0}$ detectors. The charge slopes see a NA vs FR detector dependence due to the differing electronics deadtimes in the two sets of octants.

Due to the azimuthal symmetry, the false asymmetry contribution to the experiment is suppressed when the slopes are summed over all the octants. By combining the measured beam parameter differences with the octant-summed slopes as in Eqn. 3.28, the false asymmetry induced by the HC beam parameters can be determined. Fig. 3.16 shows the total false asymmetry induced 
by helicity-correlated beam parameter differences as a function of FPD number for the $G^{0}$ forward angle measurement, and Table 3.2 shows the false asymmetries in tabular form.

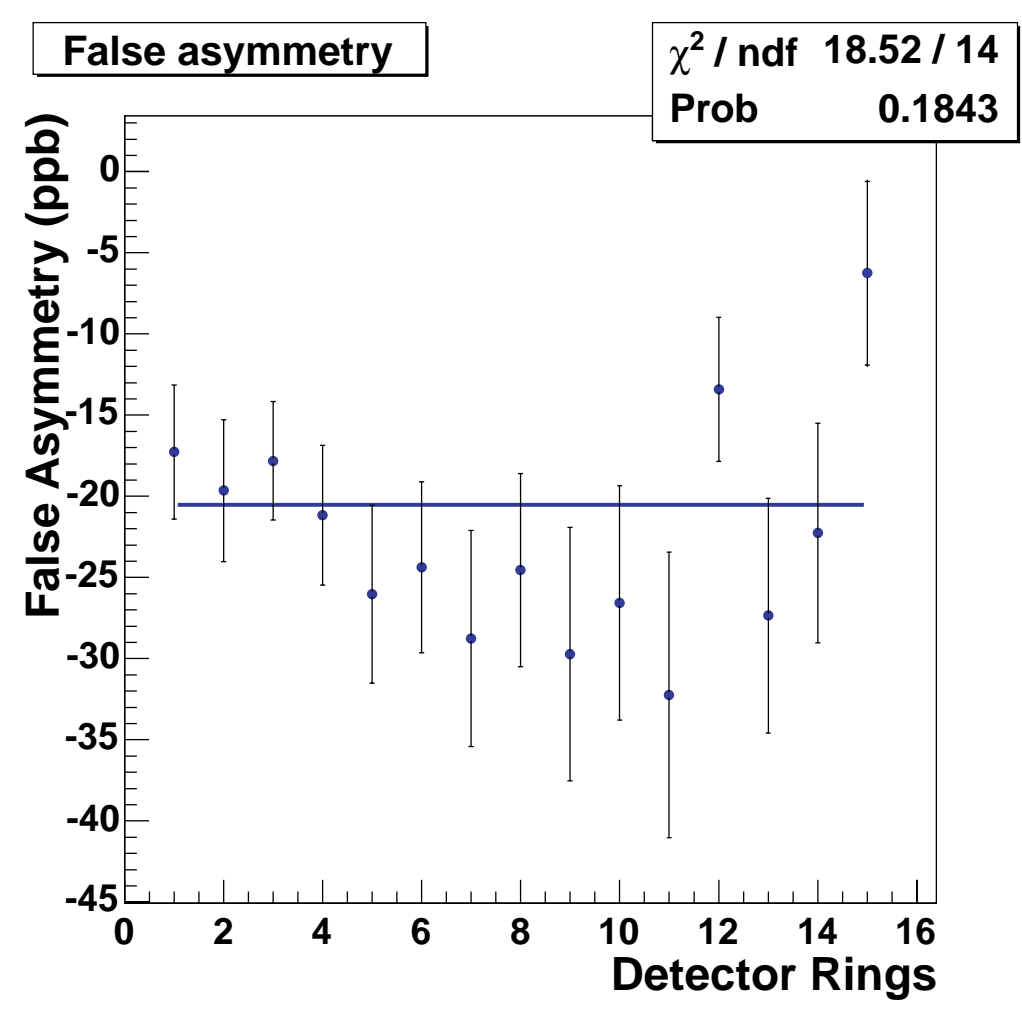

Figure 3.16: False asymmetries from multiple linear regression of HC beam difference. The slopes used to determine the false asymmetry are summed over octants.

The linear regression corrections give an average false asymmetry over all detectors of about -20 ppb, with an error of less then $10 \mathrm{ppb}$ for each detector. These corrections as well as their contributions to the overall uncertainty of the measured asymmetry are negligible.

\subsection{Background correction}

There is a significant amount of background in the region of the proton elastic peak in the tof spectrum due to inelastically scattered protons from the hydrogen target, and quasi-elastic and inelastic protons from the aluminum entrance and exit windows of the target. Typical tof asymmetry and yield spectra for all detectors in octant 2 can be seen in Appendix C.1.

Two separate studies were conducted for the purpose of background correction. First, simula- 


\begin{tabular}{|c|c|}
\hline Detector & False asymmetry (ppb) \\
\hline 1 & $-17 \pm 4$ \\
\hline 2 & $-19 \pm 4$ \\
\hline 3 & $-18 \pm 4$ \\
\hline 4 & $-21 \pm 4$ \\
\hline 5 & $-26 \pm 6$ \\
\hline 6 & $-24 \pm 5$ \\
\hline 7 & $-28 \pm 7$ \\
\hline 8 & $-24 \pm 6$ \\
\hline 9 & $-29 \pm 8$ \\
\hline 10 & $-26 \pm 7$ \\
\hline 11 & $-32 \pm 9$ \\
\hline 12 & $-13 \pm 4$ \\
\hline 13 & $-27 \pm 7$ \\
\hline 14 & $-22 \pm 7$ \\
\hline 15 & $-6 \pm 6$ \\
\hline
\end{tabular}

Table 3.2: Total false asymmetries calculated from multiple linear regression.

tion and experiment are used to understand the source of the background. Second, the data are corrected using both the measured background yield and its asymmetry.

\section{Background Contributions from the Target Cell}

In order to understand the source of the background, we have studied the backgrounds using simulations and data-taking on targets other than the standard liquid hydrogen [80] [81] [82]. The purpose is to determine both the yield and asymmetry contributions from the target cell and windows to the background yield and asymmetry.

The simulation is based on G0GEANT, and includes contributions from such in-beam elements as the entrance and exit windows of the target cell, the upstream helium cell window, and the inelastic protons from the hydrogen itself. Three types of inelastic generators were employed. One is a Lightbody and O'Connell (LBOC) [83] version modified using MAID 2003. The second is the so called "Orsay generator", developed in Orsay, France. Another generator is based on photoproduction data from the GRAAL (Grenoble Anneau Accelerateur Laser) experiment, which studies meson photo-production and the decay of excited states of the nucleon.

Data are taken with a number of auxiliary targets during the experiment. A combination of measurements is used to determine the contribution of aluminum target cell and He cell contribu- 
tions to the yield. The tof spectra are then compared to those from the simulation to ensure that the individual contributions from the various in-beam elements are understood. Once the yield contributions are understood, the asymmetry contributions from each are studied.

The tungsten radiator (equivalent in terms of radiation-length-thickness to the hydrogen target upstream exclusive of the downstream window) is used in conjunction with the flyswatter target (see Section 2.3 for description of various targets) to investigate the particular contribution from the downstream aluminum window. In this study, the electro- and photo-production of protons from the aluminum target are the same as those from the downstream window. Fig. 3.17 shows the data from the flyswatter and tungsten radiators. The simulation generally shows the correct shape, but seems to underestimate the data in the higher detectors, particularly in ranges of tof where the elastic peak usually reside (about 18 to $24 \mathrm{~ns}$, or time bins 72 to 96 ).

Detector 1

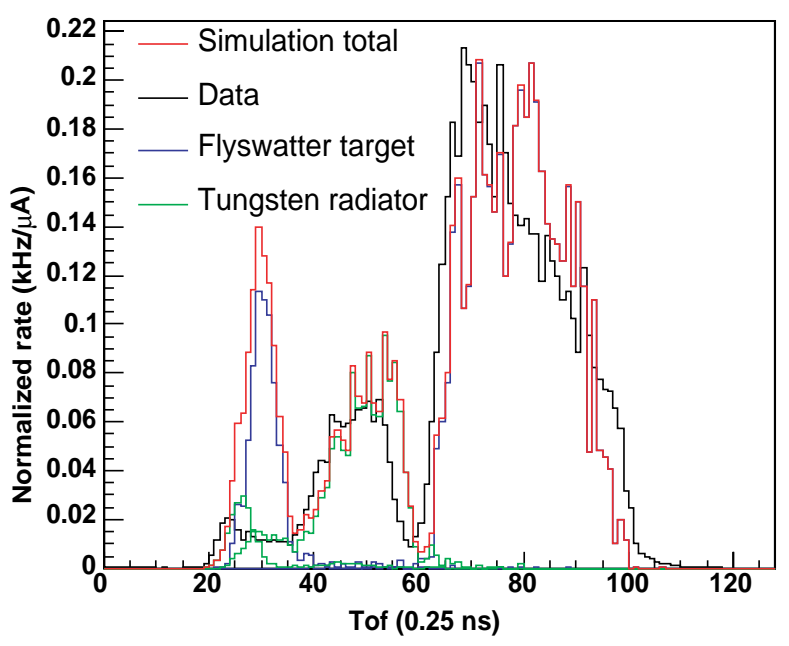

\section{Detector 14}

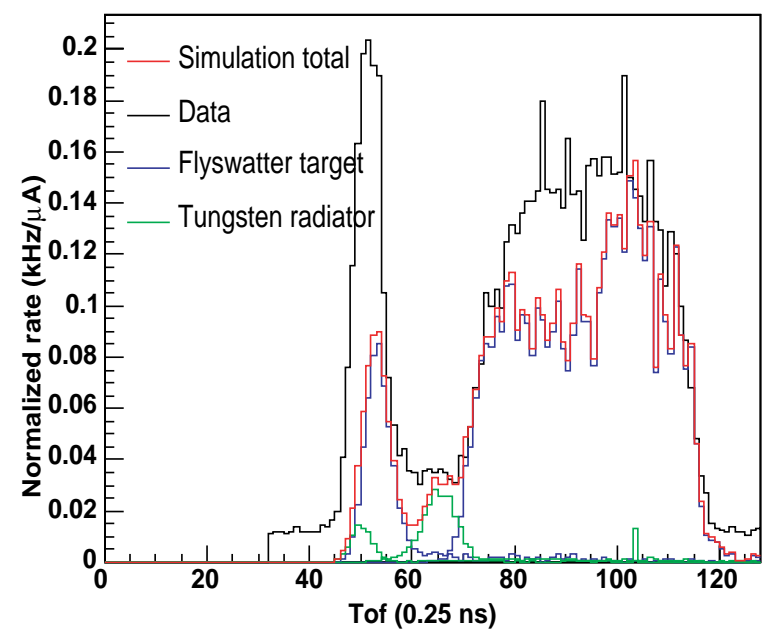

Figure 3.17: Detectors 1 and 14 flyswatter and tungsten radiator data. Simulation shows the individual contributions from the flyswatter and the tungsten radiator.

After the failure of the flyswatter target mechanism during the second commissioning run, an aluminum target near the upstream target cell window was used to measure the asymmetry of the overall proton yield from aluminum. Fig. 3.18 shows the aluminum frame data together with its simulation. The aluminum simulation looks at the individual contributions from delta production as well as quasi-deuteron (e,e'd) and quasi-free (e,e'n) particles. 
Detector 1

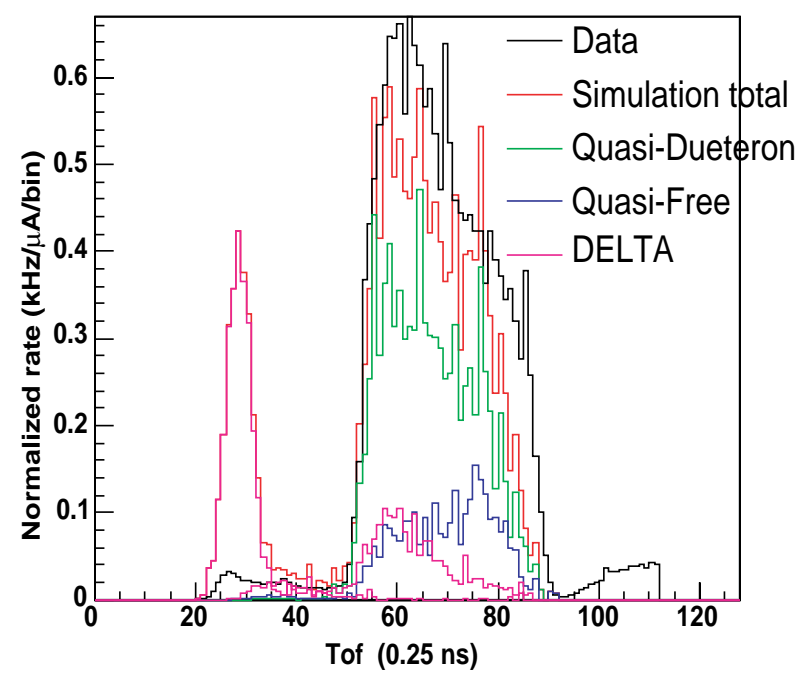

Detector 14

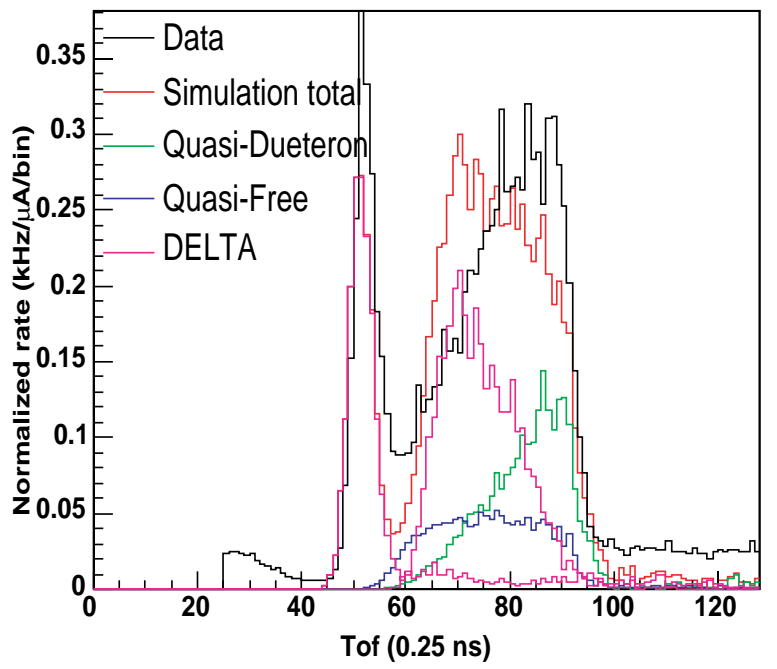

Figure 3.18: Detectors 1 and 14 aluminum frame data. In addition to the full simulation, contributions from each physical process that were investigated are shown.

"Empty" target data were taken with two different pressures of hydrogen gas in place of the liquid hydrogen in the main target cell. The merit of this type of data is that it gives insight into yield and asymmetry contributions from everything except the liquid hydrogen. The comparison between data and simulation shows that the background yield under the elastic peak is dominated by inelastic protons up to about the middle of the elastic peak, and proton electro-/photo-production (associated with pion production) from the target windows. However, the prediction of the background yield from simulation is not perfect. In particular, rates in the higher detectors are over-predicted in the LBOC generator. This is thought to originate from the fact that the MAID model is valid only for $\mathrm{W}<2 \mathrm{GeV}$, and the LBOC implementation extrapolates the MAID parameterization into a poorly measured regime. The rates seen from the MAID simulation as a function of the invariant mass, W, can be seen in Fig. 3.19. In contrast, the Orsay generator tends to underestimate the inelastic rates in the lower detectors - probably as a result of the underestimated multiple pion production - although the shapes of the tof spectra are in good agreement. Fig. 3.20 shows the LBOC and Orsay simulations in comparison with empty target data. The data shown here are determined by subtracting the target window contributions, and fitting the remaining tof spectra. The fit utilized here is the same used for the two-step fitting procedure, which will be explained 


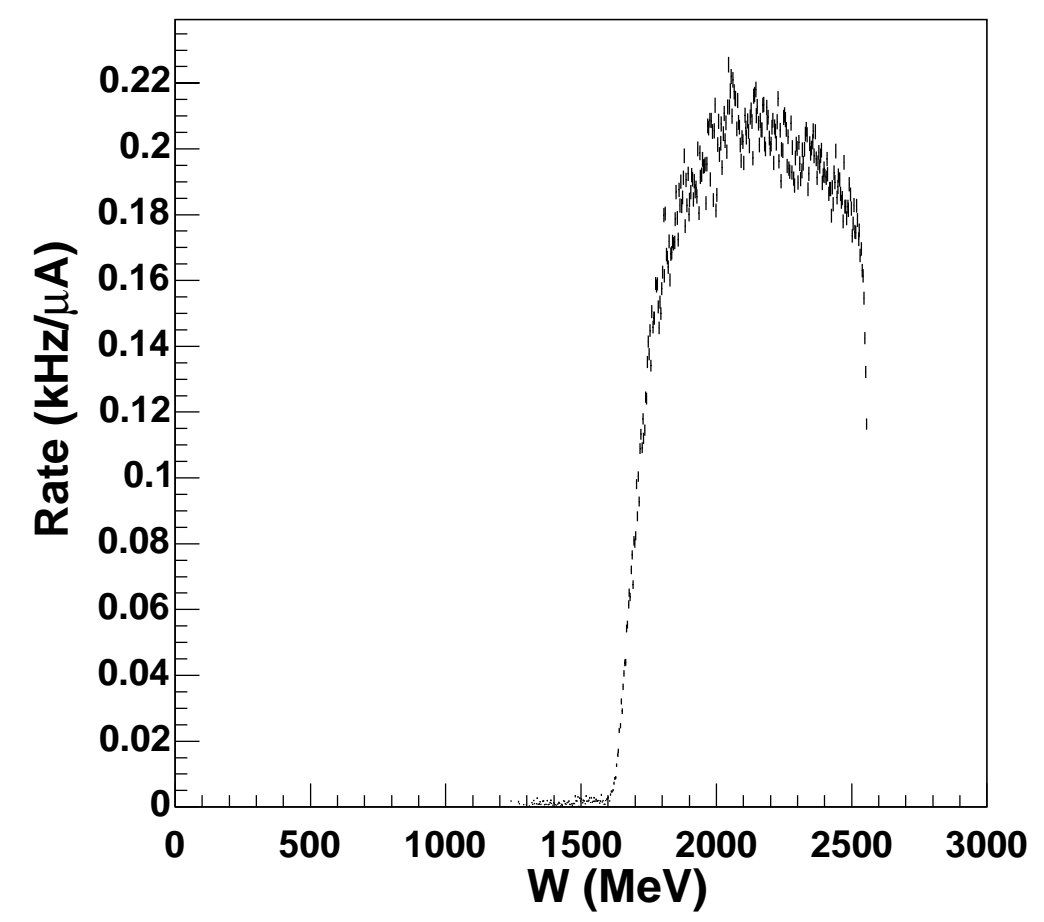

Figure 3.19: Rates vs. W seen from the MAID simulation. Detector 1 starts at the lower end (in W) of the spectrum, with Detector 10 located at about $2 \mathrm{GeV}$. The higher detectors are beyond the applicable range of MAID, and is an extrapolation from lower $\mathrm{W}$.

later in the discussion of background corrections.

The asymmetries of interest from these background studies are those under the elastic peak. Although these asymmetries cannot be simulated, their sign and size can be interpolated by observing the background asymmetries around the elastic peak (cuts 1 through 3 in Fig. 3.7). Furthermore, the asymmetries in the elastic cut for the empty target, $\mathrm{Al}$ frame, and flyswatter + tungsten radiator data give insight into their respective contributions to the background asymmetry. Fig. 3.21 shows the average asymmetries in each of these cuts for the LH2 target. The data shows positive asymmetries in cuts 1 and 2 for higher detectors, suggesting that this large positive background asymmetry is leaking into the elastic cut.

The initial hypothesis as to the source of this positive background asymmetry was the contribution of inelastic protons from the target cell and windows. This can be directly studied by taking data after removing the LH2 (as was done in the empty target runs), or by using the Al frame target. A comparison of the empty target and Al frame data, as seen in Fig. 3.22, for these cuts show agreement. However, the asymmetry contribution from these runs cannot account for the 

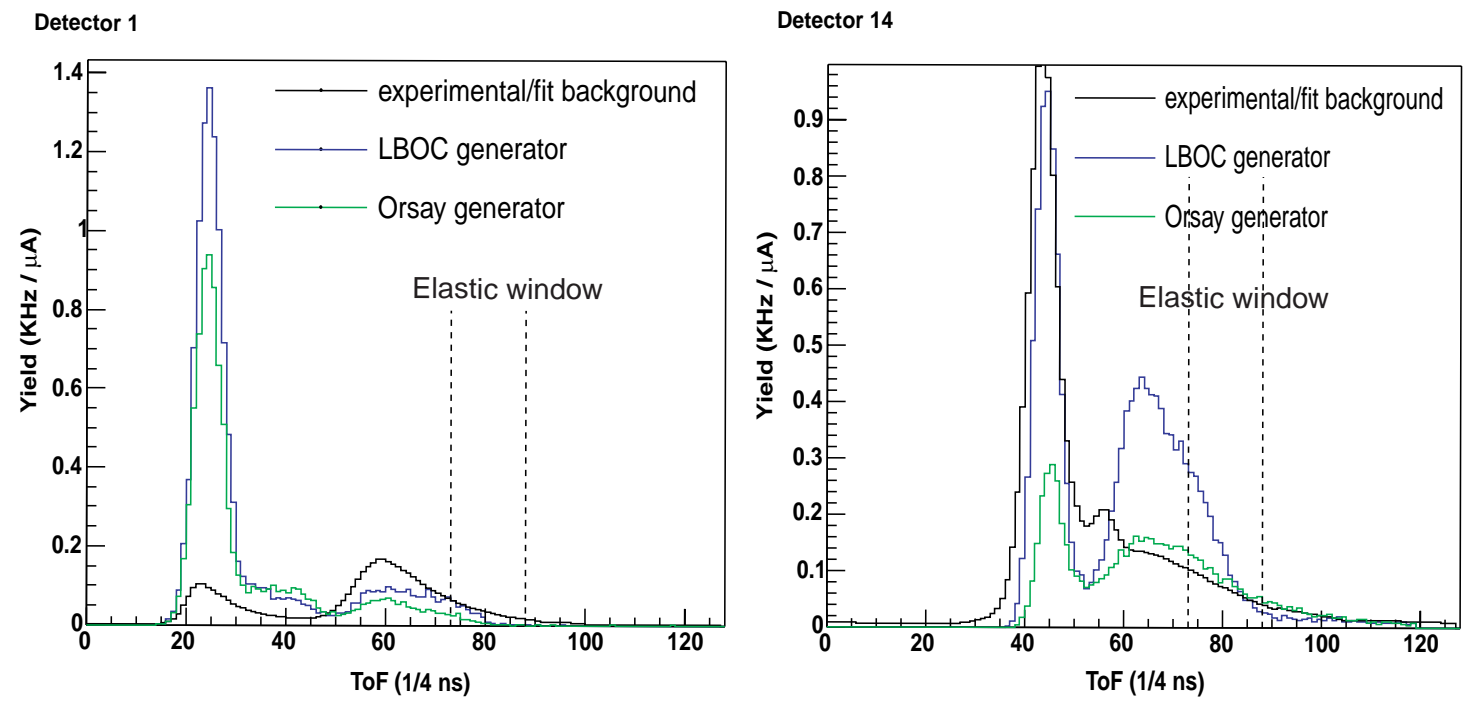

Figure 3.20: Detectors 1 and 14 empty target data and simulation. LBOC generator over estimates the background in the higher detectors, and the Orsay generator under estimates in the lower detectors. Dashed lines refer to the tof range where the elastic peak resides.

large positive asymmetries seen in Fig. 3.21. This suggests that the LH2 is directly responsible for the background asymmetry.

\section{Background Contributions from Hyperon Production}

In the end, the positive asymmetries were determined to be coming from hyperon production from the hydrogen target itself. The $\Lambda$ and $\Sigma^{+, 0}$ hyperons can be produced through electron-proton scattering via photo- or electro-production. For the $G^{0}$ forward angle kinematics, the dominant 

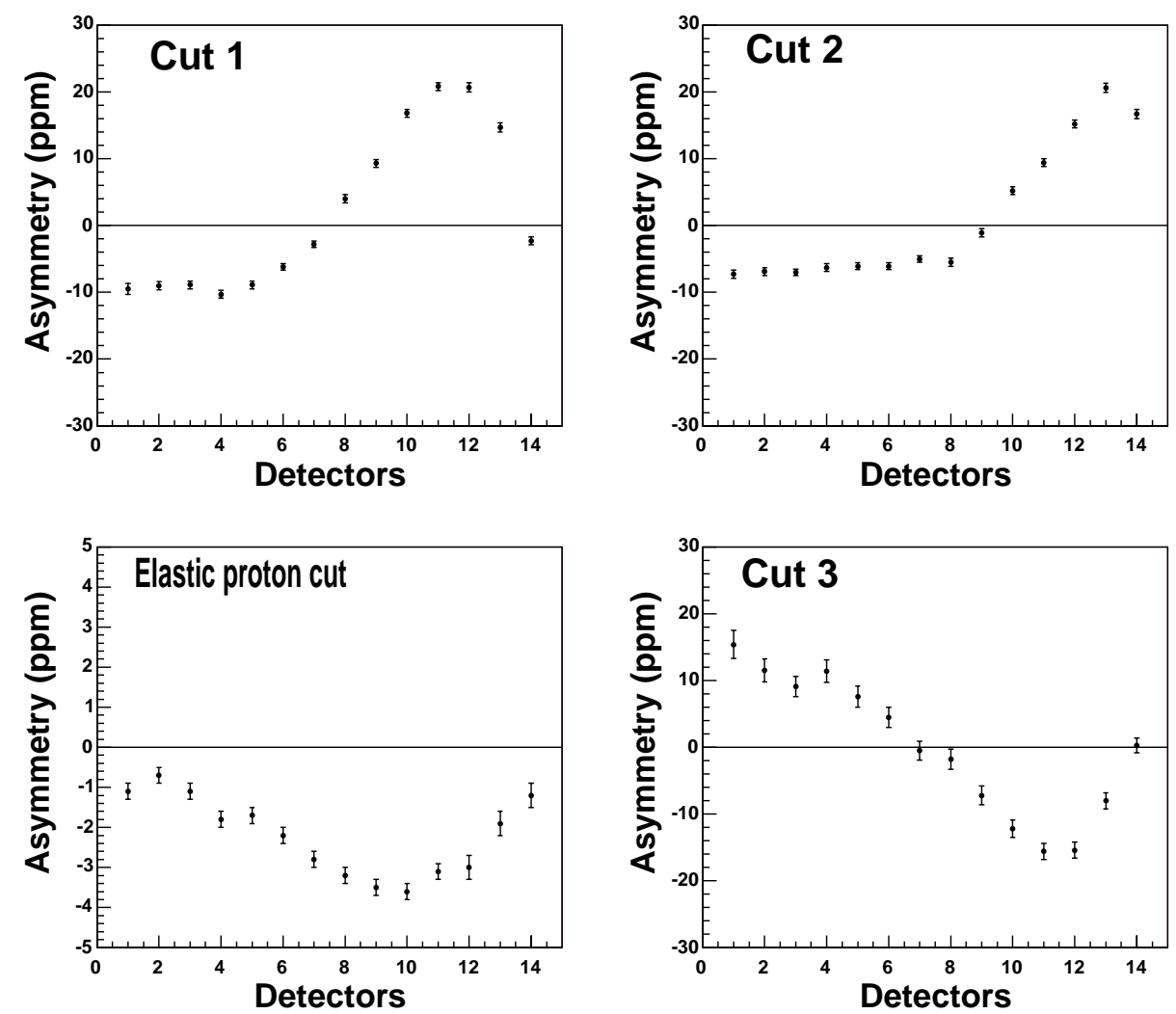

Figure 3.21: LH2 asymmetries as a function of detector. Cuts 1 and 2 show positive asymmetries for the higher detectors.

hyperon production channels are

$$
\begin{aligned}
\gamma+p \rightarrow K^{+}+ & \Lambda \\
& \left\llcorner p+\pi^{-}\right. \\
& \left\llcorner n+\pi^{0}\right. \\
\gamma+p \rightarrow K^{0}+\Sigma^{+} & \left\llcorner p+\pi^{0}\right. \\
& \left\llcorner n+\pi^{+}\right. \\
\gamma+p \rightarrow K^{+}+ & \Sigma^{0} \\
& \llcorner\Lambda+\gamma
\end{aligned}
$$



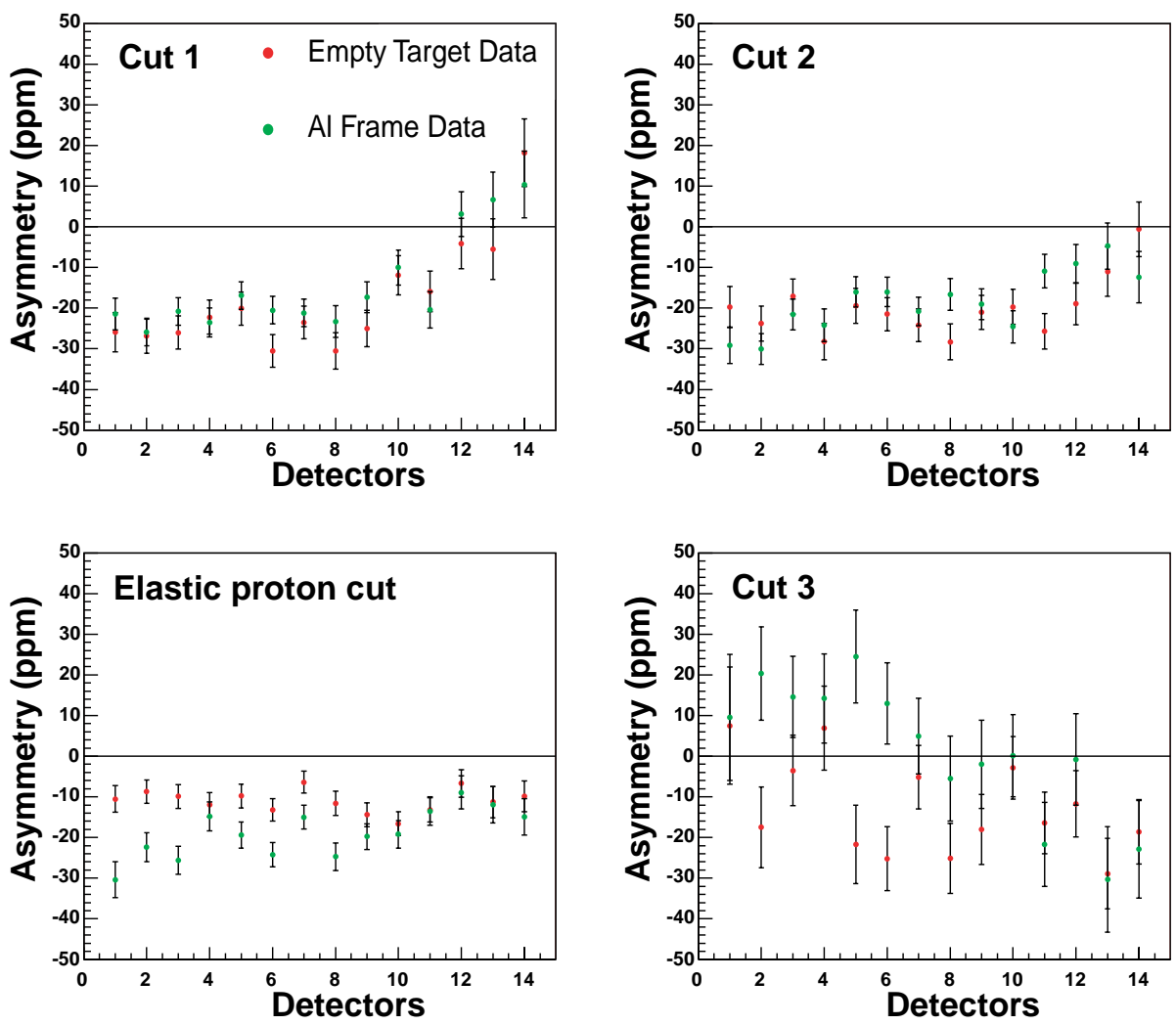

Figure 3.22: Empty target and aluminum frame data. Results show general agreement between the two sets of data. However, the asymmetries are too small to account for the large positive background asymmetries seen in the LH2 data.

with the $\Lambda$ and $\Sigma^{+}$hyperons subsequently decaying into protons and neutrons, while the $\Sigma^{0}$ decays into a $\Lambda$ before further decaying into nucleons. The $\Lambda$ decay is a weak interaction, which means it maximally violates parity (i.e. a polarized $\Lambda$ will decay preferentially in a particular direction). Therefore, we must understand how much of the beam polarization is transferred to the hyperon for this weak decay to manifest itself as a false asymmetry in our experiment.

In order to simulate the yields from hyperon production, the KAON-MAID model is used to calculate the differential cross-section of the production of hyperons, and is incorporated into G0GEANT. The results agree well with the experimental results from SAPHIR [85] as seen in Fig. 3.23. The same SAPHIR experiment has measured the hyperon polarization transfer, and was seen to be about $75 \%$.

Fig. 3.24 shows the simulated hyperon rate for the $G^{0}$ kinematics. This rate is four orders of 


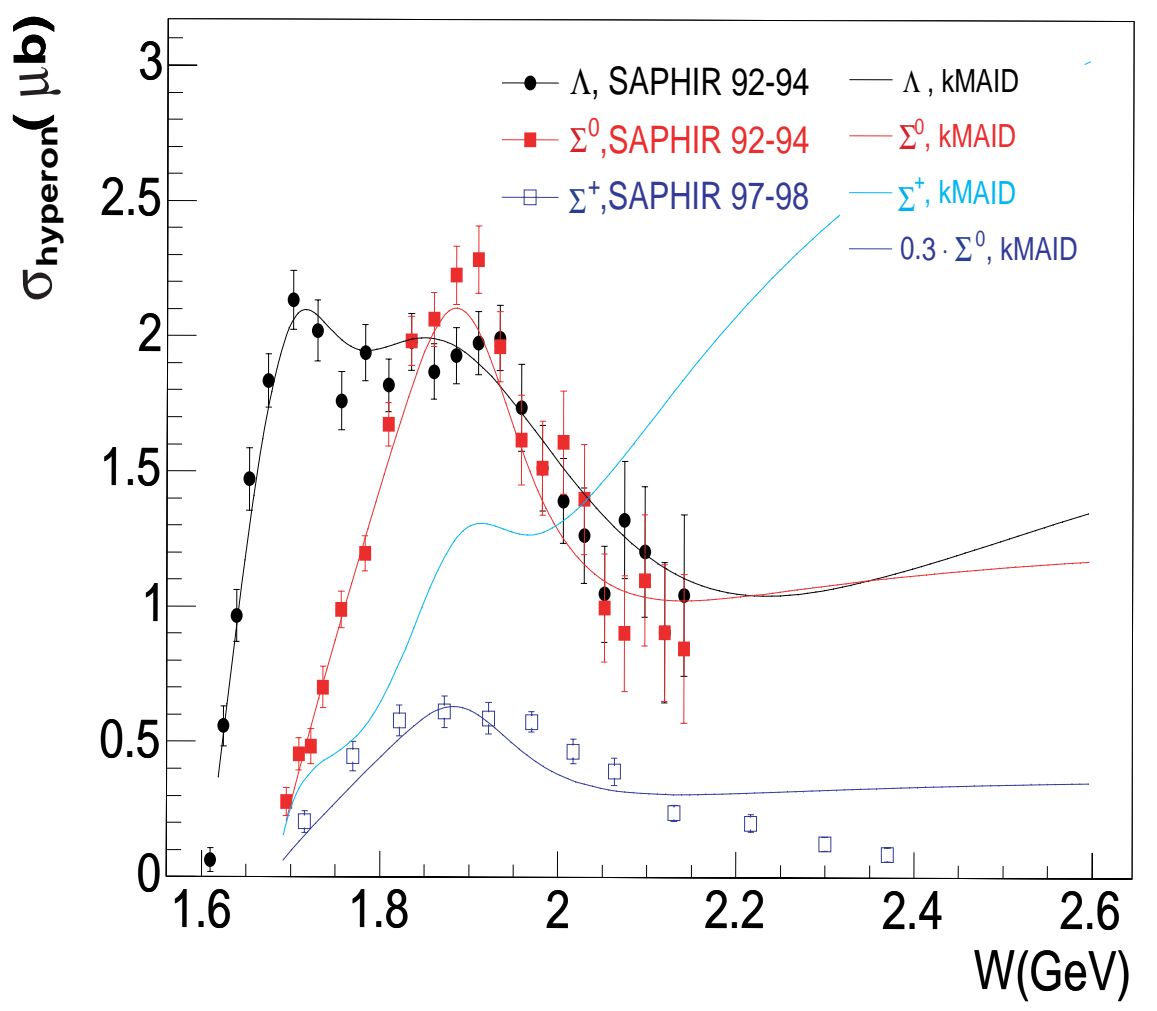

Figure 3.23: Comparison between simulation and SAPHIR data. The KAON-MAID model for the $\Sigma^{+}$hyperon is scaled by 0.3 , and reproduces the data reasonably well. The KAON-MAID model includes parameters that are determined empirically. The SAPHIR data for the $\Sigma^{+}$shown here are more recent than the KAON-MAID model, which is the reason for the initial discrepancy.

magnitude smaller than the total rate seen in each detector. However, the asymmetry (of $75 \%$ ) is large enough such that the hyperons produce a large positive background asymmetry.

A simulation of the hyperon asymmetry is performed through G0GEANT by considering the three scattering planes involved in the creation and decay of the hyperon. They include the plane with the incident and scattered electron (electron plane), the plane with the virtual photon and the hyperon (hyperon production plane), and the plane (hyperon decay plane) which consists of the decay products (the nucleon and the pion). The asymmetry can be written as

$$
A=\alpha P_{b} P_{z}^{\prime} \cos \left(\theta_{\hat{z}, \overrightarrow{k_{N}}}^{R F}\right)
$$




\section{Detector 14}

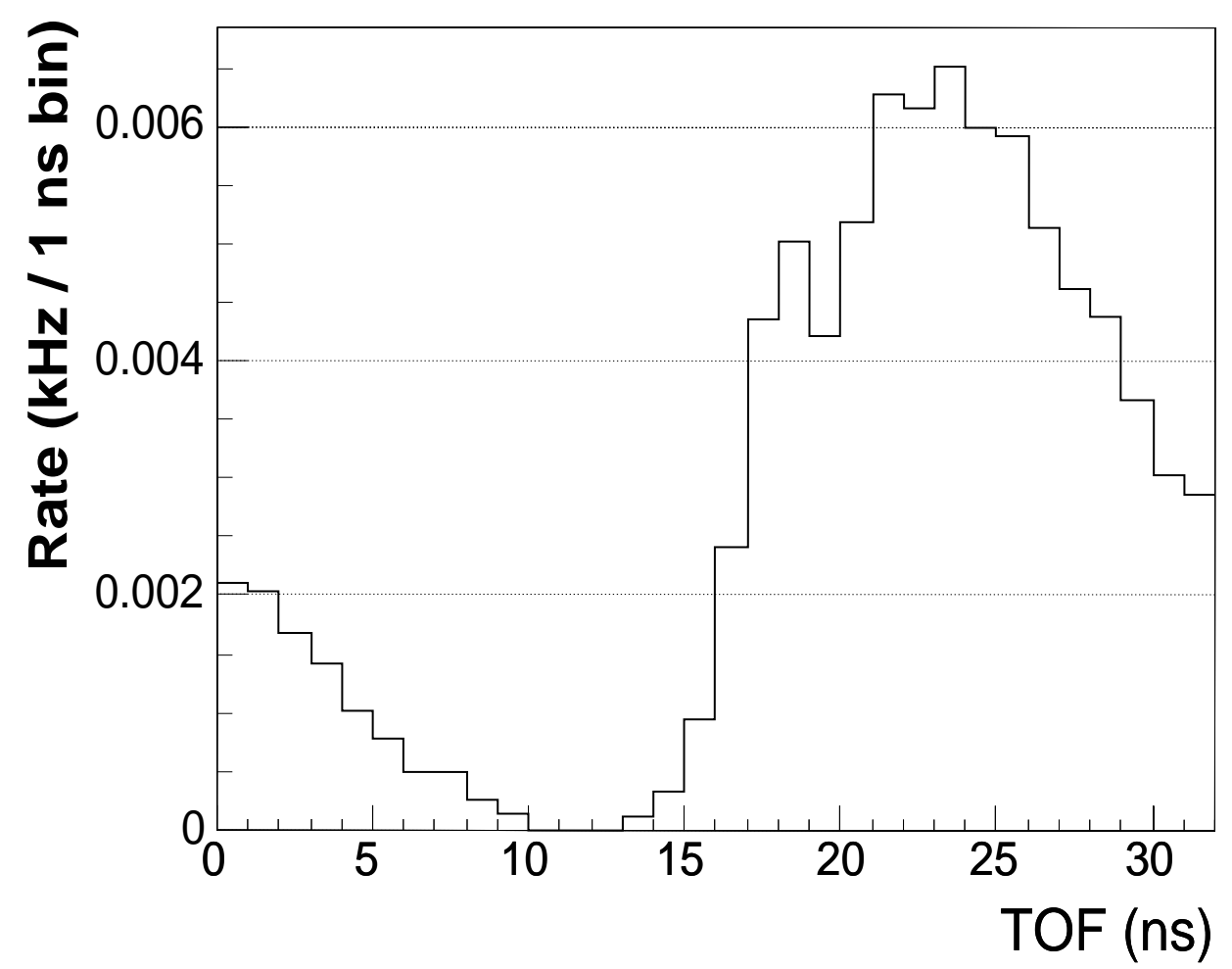

Figure 3.24: Proton rate from hyperon decay simulated through G0GEANT. The rate under the elastic peak $(\sim 18-25 \mathrm{~ns})$ is four orders of magnitude smaller than the elastic rate.

where $\alpha$ characterize the parity-violating nature of the hyperon decay, $P_{b}$ is the beam polarization, $P_{z}^{\prime}$ is the polarization transferred to the virtual photon along the virtual photon momentum, and $\theta_{\hat{z}, \overrightarrow{k_{N}}}^{R F}$ is the angle between the momentum of the hyperon and that of the nucleon which results from its decay (in the reference frame of the hyperon) [86] [87]. The polarization of the hyperon is implicitly included in Eqn. 3.35 due to studies in [88] from which we deduce that the polarization transfer from the virtual photon to the hyperon is estimated to be about 100\%. An asymmetry can be calculated by incorporating Eqn. 3.35 into G0GEANT, and the results can be weighed by the hyperon yield to generate an asymmetry spectrum. The results can be seen in Fig. 3.25. In detector 14, the effect of the hyperon asymmetry can clearly be seen. In the 18 to 25 ns region where the elastic peak resides, the hyperon asymmetry has a positive peak, whereas the elastic asymmetry is expected to be negative and of order 10's of ppm. 

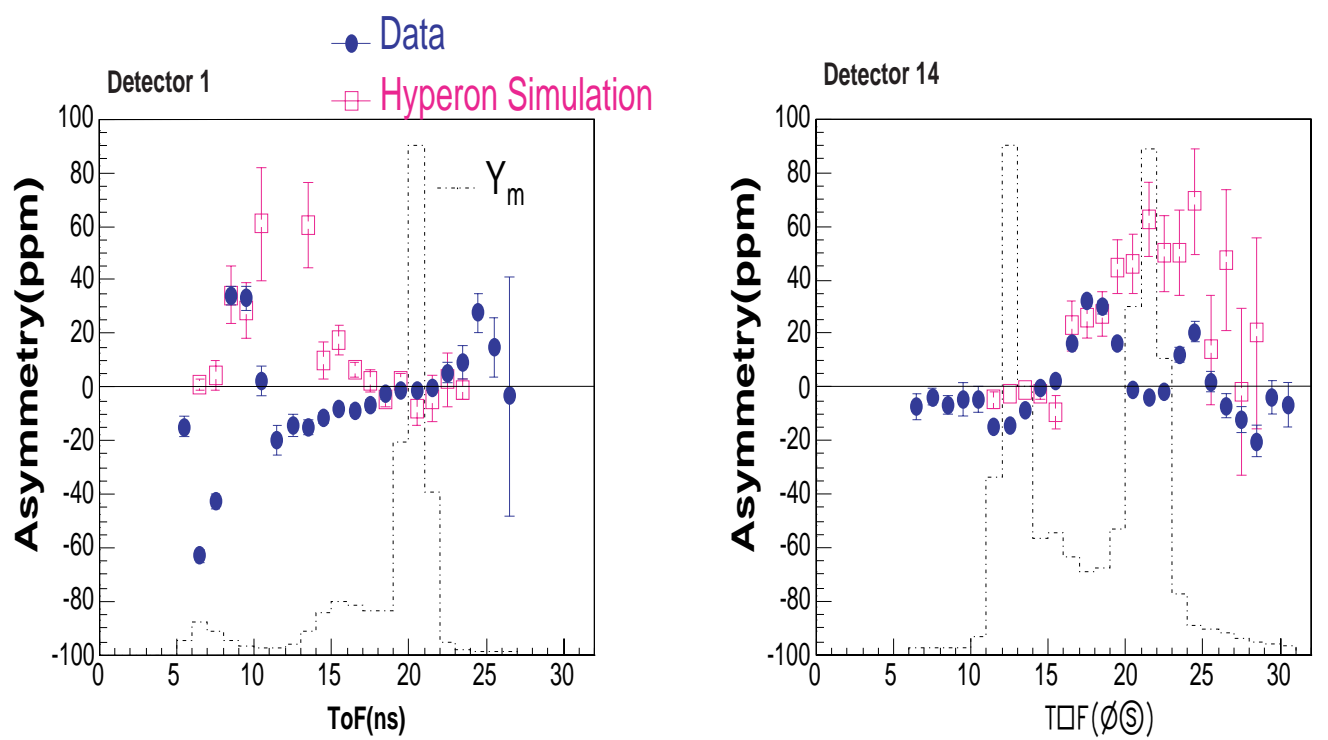

Figure 3.25: Raw measured asymmetries and simulated hyperon asymmetries.

\section{Background Correction}

The investigations of the background yield and asymmetry described above were undertaken to show that we understand the sources of the background and the possible range of their sizes in the region of the elastic peak in the tof spectrum. However, the actual background correction to the elastic asymmetry is made using only data from nearby regions of the tof spectrum as measured in the main experiment. The auxiliary measurements are used principally to inform the estimates of the systematic uncertainties associated with the background correction.

The background correction scheme must eliminate both the dilution effect and the background asymmetry from the elastic proton peak. The contributions of the background yield and asymmetry to the parity-violating physics asymmetry can be expressed in terms of measured and background rates and asymmetries

$$
A_{\text {meas }}(t)=\frac{Y_{e l}(t) A_{e l}(t)+Y_{b k g}(t) A_{b k g}(t)}{Y_{e l}(t)+Y_{b k g}(t)}=\left(1-f_{b}(t)\right) A_{e l}(t)+f_{b}(t) A_{b k g}(t)
$$

where $Y_{e l}(t)$ and $A_{e l}(t)$ are the elastic yields and asymmetries, $Y_{b k g}(t)$ and $A_{b k g}(t)$ are the background yields and asymmetries, and the background fraction, $f_{b}(t)$, is defined as the ratio between the background yield and the total yield. The correction of the background asymmetry is performed using a two-step elastic sideband fitting procedure. However, the specific implementation 
depends on the detector. Since there are four tof distributions that must be determined $\left(A_{e l}(t)\right.$, $Y_{e l}(t), A_{b k g}(t)$, and $\left.Y_{b k g}(t)\right)$, a choice must be made in terms of what functional form should be used for each. A significant difference exists in the choice of such functions suggested by the different shapes of the low and high $Q^{2}$ data. Detectors 1-14a use one set of functional forms, and 15a, 15b, $15 \mathrm{c}$, and $14 \mathrm{~b}$ use their own set of models to parameterize the yield. Here, 14a and 14b refer to the low and high $Q^{2}$ elastic peaks in detector 14 (Fig. C.14), and 15a, 15b, and 15c refer to the three $Q^{2}$ bins in detector 15 (Fig. C.15).

\section{Detectors 1 through 14a}

The first step in the two-step fitting procedure involves determining $Y_{e l}(t)$ and $Y_{b k g}(t)$ by fitting the yield distribution with

$$
Y_{\text {total }}(t)=Y_{e l}(t)+Y_{b k g}(t)
$$

where $Y_{\text {total }}(t)$ is the total measured yield. In such a fit, a functional form is chosen for $Y_{e l}(t)$ and $Y_{b k g}(t)$, and the fit is allowed to determine the appropriate parameters for each function. As seen in Appendix C.1, detectors 1 to 14 all show elastic yield peaks that are well represented by Gaussians. Therefore, for those detectors, a gaussian is chosen for $Y_{e l}(t)$, and $Y_{b k g}(t)$ is modeled with a fourth order polynomial. The fitting range is chosen such that a large portion of the background region is included allowing the best interpolation of the background yield under the elastic peak. For detectors 1-14a, the fitting range is $7 \mathrm{~ns}$ below the elastic window to $5 \mathrm{~ns}$ above the elastic window. A sample fit of the yields can be seen in Fig. 3.26. For the purpose of the fit, the error on the yields is the standard deviation of the yields over runs and is not determined from counting statistics. The reason is equivalent to that given for the uncertainty of the linear regression slopes. With the large count rate in our detectors, the statistical uncertainty is five to six orders of magnitude smaller than the yields themselves. Therefore, the uncertainty in the yields is dominated by systematics. We therefore estimate this systematic uncertainty by observing the variation of the yields over time. From the fit, the background fraction averaged over the elastic range can be calculated

$$
<f_{b}>=\frac{\int_{e l 1}^{e l 2} Y_{b k g}(t) d t}{\int_{e l 1}^{e l 2}\left(Y_{e l}(t)+Y_{b k g}(t)\right)}
$$


Octant 2 Detector 8

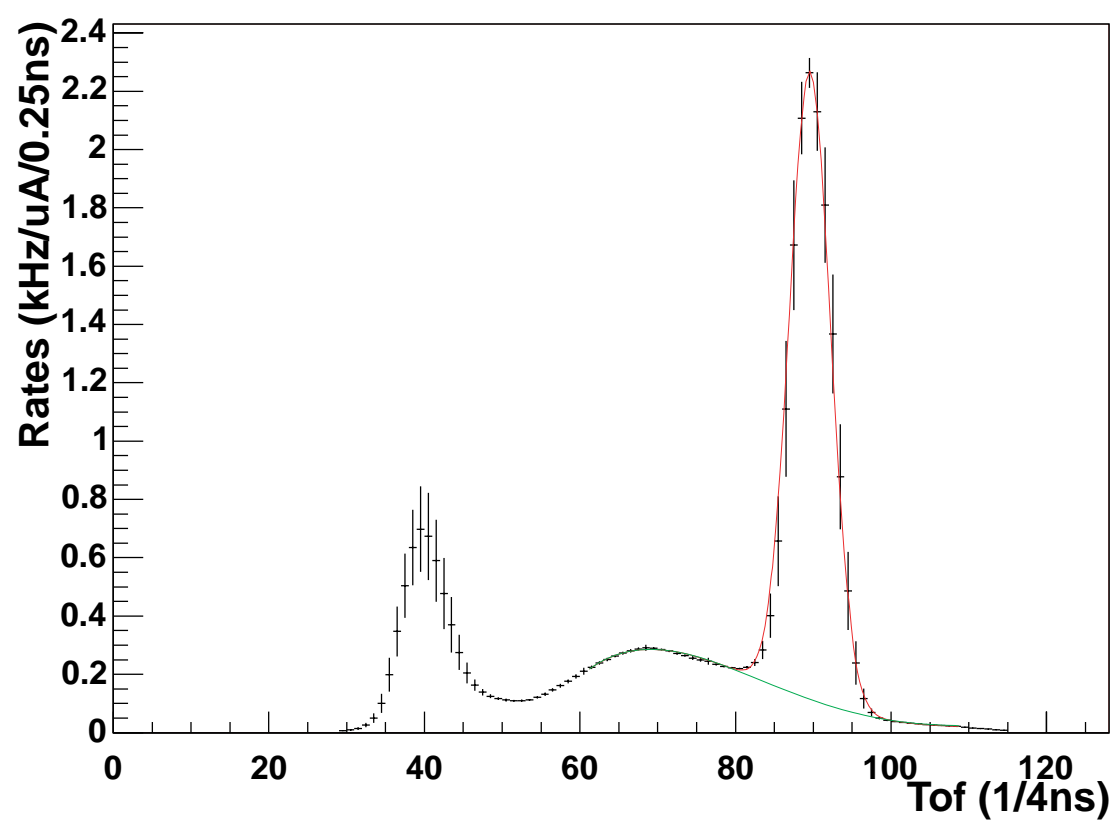

Figure 3.26: Yield fit for octant 2, detector 8. The red curve shows the combined gaussian $Y_{e l}(t)$ + fourth order polynomial $Y_{b k g}(t)$ fit. The green curve is the fourth order polynomial background yield.

where $e l 1$ and $e l 2$ are the low and high limits of the elastic time window. Once the background fraction is determined, the second step of the procedure involves fitting the asymmetry distribution using Eqn. 3.36. Once again, a suitable functional form must be chosen for $A_{e l}(t)$ and $A_{b k g}(t)$. Since one average elastic asymmetry value is measured for each detector for FPDs 1-14a, $A_{e l}(t)$ is modeled as a constant. The choice of the functional form for the background asymmetry is made based on the shape and behavior of the asymmetry between the pion and elastic peaks.

The positive background asymmetry peak moves from the higher edge of the pion peak toward the elastic proton peak as the detector number is counted up, and completely overlaps the elastic peak in detectors 14 and 15. In order to model this bump, a quadratic form $\left(A_{b k g}(t)=p 1+p 2 t+p 3 t^{2}\right.$, where $p 1, p 2$, and $p 3$ are coefficients to be determined by fitting the measured asymmetry) is used to fit the background asymmetry. Fig. 3.27 shows a sample fit of the asymmetry.

Table 3.3 shows the quality of the fits for all 14 detectors. With the exception of detector 14a, all fits show good reduced $\chi^{2}$. Whereas only one $Q^{2}$ elastic peak is fit for detectors $1-13$, the fitting 


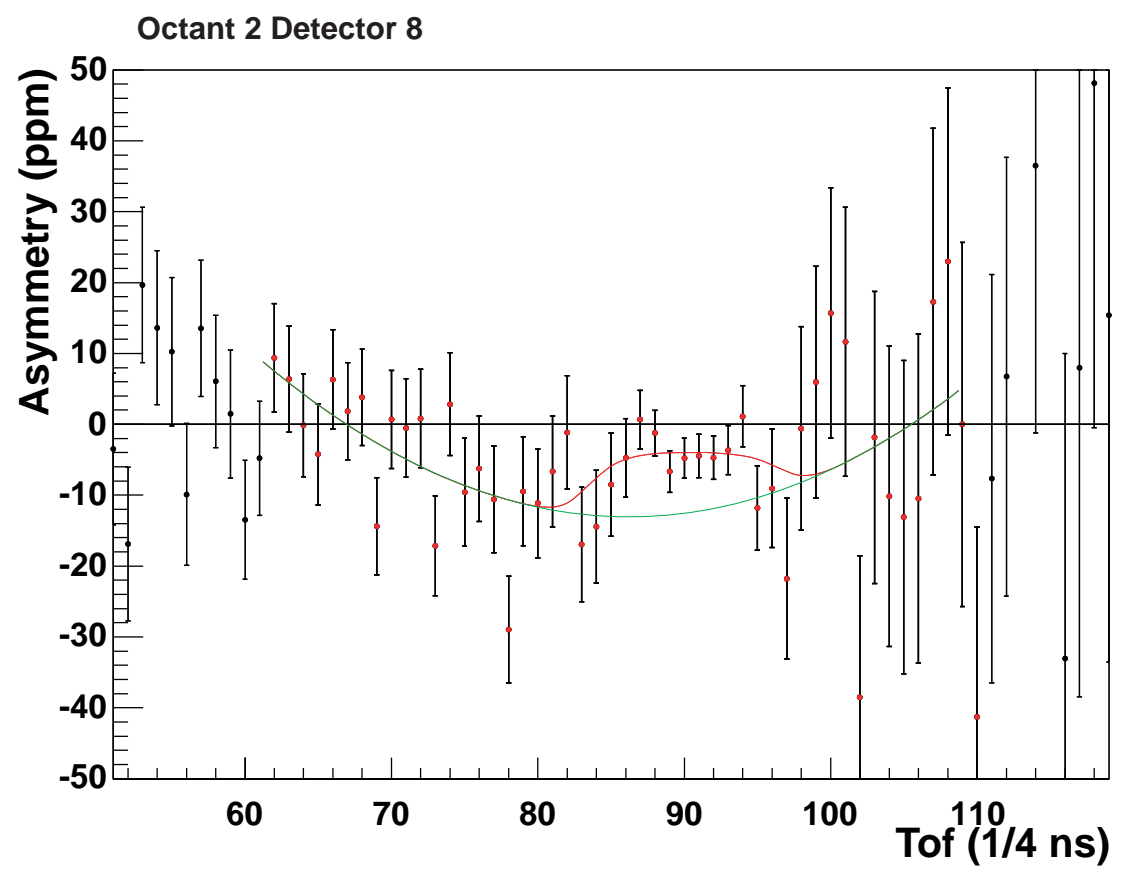

Figure 3.27: Asymmetry fit for octant 2, detector 8. The red curve shows the combined constant $A_{e l}+$ quadratic $A_{b k g}$ fit. The green curve is the quadratic background asymmetry. The red points are data included in the fit.

in detector 14 is complicated by the existence of a second elastic peak. In this detector, the higher $Q^{2}$ peak (which is lower in tof; see Fig. C.14 in Appendix C.1) is treated as a background for the purpose of fitting the lower $Q^{2}$ peak. However, a simple polynomial may not be sufficient to characterize the shape of the yield or the asymmetry distribution in this detector, resulting in a larger $\chi^{2}$. The background fractions and asymmetries extracted from this method can be found in Table C.5 in Appendix C.3, where all of the correction factors needed to determine the final parity-violating asymmetry can also be found.

In order to determine the uncertainty, we re-write Eqn. 3.36

$$
A_{e l}=\frac{A_{\text {meas }}-f_{b} A_{b k g}}{1-f_{b}}
$$

to derive the elastic asymmetry. The error on the asymmetry, $\delta A_{e l}$, can thus be written as a sum 


\begin{tabular}{|c|c|}
\hline Detector & $\chi^{2} / \nu$ \\
\hline 1 & $236 / 208$ \\
\hline 2 & $265 / 208$ \\
\hline 3 & $193 / 208$ \\
\hline 4 & $209 / 208$ \\
\hline 5 & $232 / 208$ \\
\hline 6 & $221 / 208$ \\
\hline 7 & $176 / 208$ \\
\hline 8 & $213 / 208$ \\
\hline 9 & $236 / 208$ \\
\hline 10 & $208 / 208$ \\
\hline 11 & $207 / 208$ \\
\hline 12 & $202 / 208$ \\
\hline 13 & $228 / 208$ \\
\hline $14 \mathrm{a}$ & $336 / 208$ \\
\hline
\end{tabular}

Table 3.3: Reduced $\chi^{2}$ from the asymmetry fits for detectors 1-14a.

of four terms

$$
\delta A_{e l}^{2}=\left(\frac{\delta A_{\text {meas }}}{1-f_{b}}\right)^{2}+\left(\frac{\left(A_{\text {meas }}-A_{b k g}\right) \delta f_{b}}{\left(1-f_{b}\right)^{2}}\right)^{2}+\left(\frac{f_{b} \delta A_{b k g}}{1-f_{b}}\right)^{2}+\frac{2\left(A_{\text {meas }}-A_{b k g}\right)}{\left(1-f_{b}\right)^{2}} \delta_{f_{b}, A_{b k g}}
$$

where $\delta A_{\text {meas }}, \delta A_{b k g}$, and $\delta f_{b}$ are uncertainties on the measured asymmetry, the background asymmetry, and the background fraction, and $\delta_{f_{b}, A_{b k g}}$ is the covariance of the background fraction and asymmetry. This covariance term arises from the fact that the two-step fitting procedure treats the yield and asymmetry distributions separately, and does not account for their correlation. For simplicity, we will define

$$
\begin{aligned}
& \delta A_{1}=\frac{\delta A_{\text {meas }}}{1-f_{b}} \\
& \delta A_{2}=\frac{\left(A_{\text {meas }}-A_{b k g}\right) \delta f_{b}}{\left(1-f_{b}\right)^{2}} \\
& \delta A_{3}=\frac{f_{b} \delta A_{b k g}}{1-f_{b}} \\
& \Delta=\frac{2\left(A_{\text {meas }}-A_{b k g}\right)}{\left(1-f_{b}\right)^{2}} \delta_{f_{b}, A_{b k g}}
\end{aligned}
$$

such that Eqn. 3.40 can be re-written as

$$
\delta A_{e l}{ }^{2}=\delta A_{1}^{2}+\delta A_{2}^{2}+\delta A_{3}^{2}+\Delta
$$


The fit directly outputs the elastic asymmetry with an error. However, this error not only includes the statistical uncertainty of the measured asymmetry, but is also related to the precision with which the sideband asymmetries are measured. Therefore, instead of using the error from the fit, the statistical uncertainty is calculated from counting statistics of the elastic and background asymmetries as discussed below, while the systematic uncertainty is determined by a separate study.

We first separate $\delta A_{3}$ as

$$
\delta A_{3}^{2}=\delta A_{3, s t a t}^{2}+\delta A_{3, s y s}^{2}
$$

where $\delta A_{3, \text { stat }}$ is the statistical uncertainty, and $\delta A_{3, s y s}$ is the systematic uncertainty. Since the counting rate is large in this experiment, the yield and the background fraction $f_{b}$, both have small statistical uncertainties. Therefore, the uncertainty on $f_{b}$ is dominated by its systematic uncertainty, and we safely neglect its statistical uncertainty.

We can derive from Eqn. 3.40

$$
\delta A_{e l, s t a t}=\sqrt{\delta A_{1}^{2}+\delta A_{3, \text { stat }}^{2}}
$$

where $\delta A_{e l, s t a t}$ is the statistical uncertainty of the elastic asymmetry. $\delta A_{3, \text { stat }}$ can be determined from counting statistics as

$$
\delta A_{3, \text { stat }}=\frac{f_{b}}{1-f_{b}} \delta A_{b k g, s t a t}=\frac{f_{b}}{1-f_{b}} \frac{1}{\sqrt{f_{b}}} \delta A_{\text {meas }}
$$

so Eqn. 3.44 reduces to

$$
\delta A_{e l, s t a t}=\frac{\sqrt{1+f_{b}}}{1-f_{b}} \delta A_{\text {meas }}
$$

where we have now determined the statistical uncertainty on the elastic asymmetry simply from counting statistics.

The systematic uncertainty on the elastic asymmetry is, from Eqn. 3.42,

$$
\delta^{2} A_{e l, s y s}=\delta A_{2}^{2}+\delta A_{3, s y s}^{2}+\Delta
$$

where we will, again, neglect the statistical uncertainty of $f_{b}$, and the contribution from $\delta A_{3}$ here 
does not include the statistical contribution as described above.

In order to determine the systematic error contributions from the background fraction $\left(\delta A_{2}\right)$ and the covariance term $(\Delta)$, we vary $Y_{b k g}(t)$ in the first step of the two-step fitting procedure, and observe the variance in $A_{e l}$. We constrain the shape of $Y_{b k g}(t)$ by creating a lozenge as seen in Fig. 3.28 with two of its opposite ends fixed at the background yields as measured at the ends of the elastic time window. Points are randomly chosen along a vertical line through the center of the lozenge, and each point is connected, by two straight lines with the background yields measured at the elastic cut boundaries and used as a model of $Y_{b k g}(t)$. This is iterated $\mathrm{N}$ times, and $A_{e l}$ is determined through the two-step fit for each choice of this $Y_{b k g}(t)$. The spread in the resulting

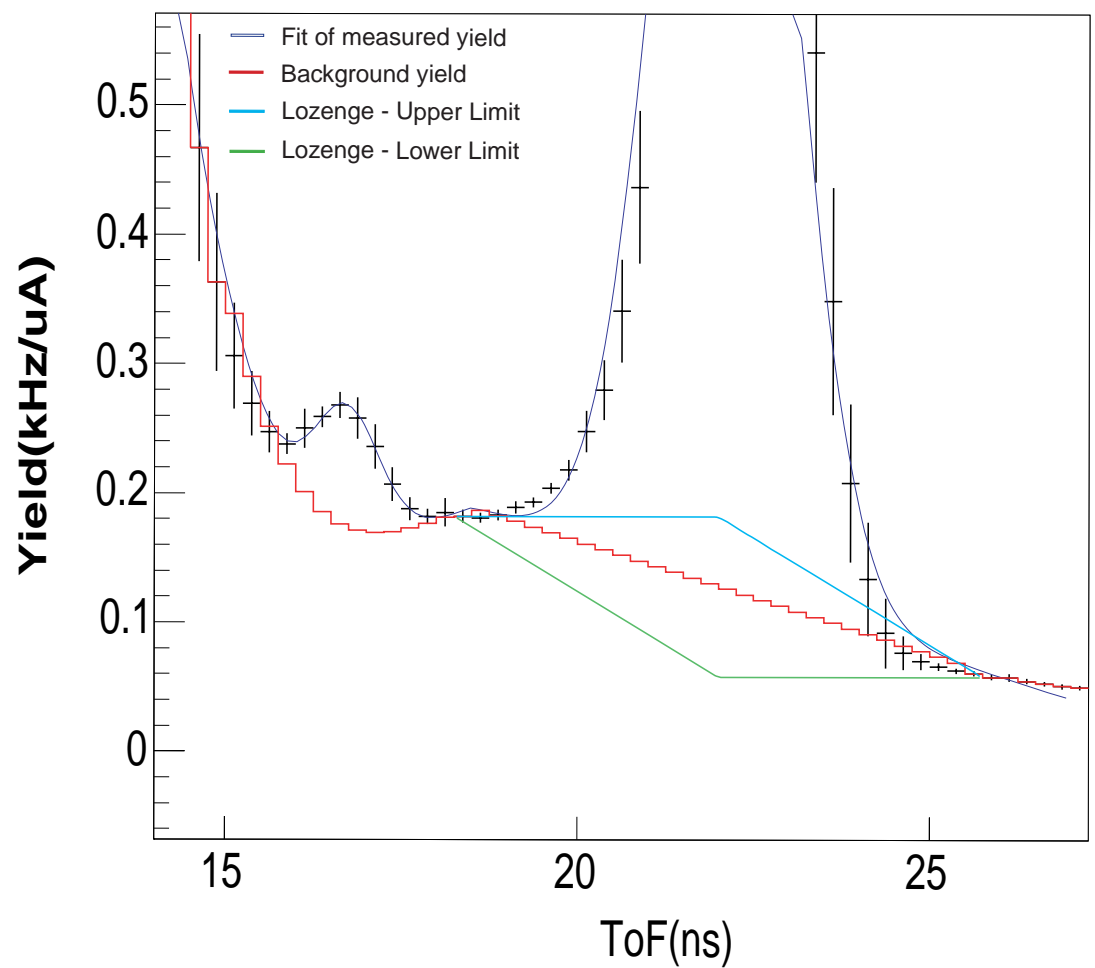

Figure 3.28: A lozenge shape is chosen as a conservative estimate of the upper and lower bounds of the shape of $Y_{b k g}(t)$. We allow $Y_{b k g}(t)$ to vary within this lozenge (as described in the text) in order to see the effect of such variations on the elastic asymmetry.

elastic asymmetry has the contributions from $\delta A_{2}$ and $\Delta$ naturally built in. However, in addition 
to $\delta A_{2}^{2}$ and $\Delta$ seen in Eqn. 3.47, one additional term, $A_{\text {induced }}$, contributes to this variance

$$
\delta^{2} A_{\text {el,variance }}=\delta A_{2}^{2}+\Delta+\delta A_{\text {induced }}^{2}
$$

where $\delta A_{\text {el,variance }}$ is the variance of the elastic asymmetry, and $\delta A_{\text {induced }}$ is the induced change in $A_{b k g}$ due to variations in $\delta f_{b}$ (whereas $\Delta$ is due to variations in $f_{b}$ ). Table 3.4 show the contributions from each term found from this study.

The term in Eqn. 3.47 associated with the systematic uncertainty of the background asymmetry, $\delta A_{3, s y s}^{2}$, is determined by varying both the functional form of the $A_{b k g}(t)$ and the range of time bins of the asymmetry fit. The spread of $A_{e l}$ resulting from these variations determines the contribution of the model-dependent uncertainties of $A_{b k g}(t)$. However, since the choice of the functional forms is somewhat arbitrary, the spread is calculated by weighing each value of $A_{e l}$ by the confidence of the fit. The resulting values of $\delta A_{3, \text { sys }}^{2}$ are also shown in Table 3.4. From Eqn. 3.42 and 3.48, we

\begin{tabular}{|c|c|c|c|c|c|}
\hline Detector & $\Delta$ & $\delta A_{\text {induced }}$ & $\delta A_{2}$ & $\delta A_{\text {el,variance }}$ & $\delta A_{3, \text { sys }}^{2}$ \\
\hline 1 & -0.0011 & 0.0387 & 0.041 & 0.059 & 0.16 \\
\hline 2 & -0.0003 & 0.0463 & 0.011 & 0.048 & 0.05 \\
\hline 3 & -0.0031 & 0.0530 & 0.033 & 0.027 & 0.06 \\
\hline 4 & -0.0125 & 0.0656 & 0.103 & 0.054 & 0.19 \\
\hline 5 & -0.0130 & 0.0759 & 0.094 & 0.049 & 0.22 \\
\hline 6 & -0.0338 & 0.1065 & 0.164 & 0.068 & 0.24 \\
\hline 7 & -0.0158 & 0.0562 & 0.152 & 0.011 & 0.17 \\
\hline 8 & -0.0353 & 0.1030 & 0.176 & 0.094 & 0.15 \\
\hline 9 & -0.0348 & 0.0961 & 0.188 & 0.105 & 0.16 \\
\hline 10 & 0.0054 & 0.0543 & 0.061 & 0.101 & 0.20 \\
\hline 11 & -0.0065 & 0.0944 & 0.039 & 0.051 & 0.09 \\
\hline 12 & -0.2142 & 0.1637 & 0.670 & 0.041 & 0.25 \\
\hline 13 & -0.2852 & 0.1680 & 0.869 & 0.059 & 0.33 \\
\hline $14 \mathrm{a}$ & -0.4792 & 0.2514 & 0.982 & 0.069 & 0.29 \\
\hline
\end{tabular}

Table 3.4: Contributions to the the systematic uncertainties for detectors 1-14a.

get

$$
\delta A_{\text {el,sys }}^{2}=\delta A_{\text {el,variance }}^{2}+\delta A_{3, s y s}^{2}-\delta A_{\text {induced }}^{2}
$$

where we subtract $\delta A_{\text {induced }}$ to avoid the contributions from this term.

Finally, we make a separation of systematic uncertainties that are unique to each detector (point-to-point uncertainties) from those that globally affect all detectors (correlated uncertainties). 
If, for example, the functional form of $Y_{b}$ or $A_{b k g}$ changes globally across all detectors, this will cause systematic uncertainties that are correlated across these detectors. We make an estimate of the separation based on the various functional forms used for both the background yield and asymmetry. As detailed above, we observe the variance of $A_{e l}$ as the models of the background yield and asymmetries are changed. From the various fits, we observe that the first and third order polynomial models of $Y_{b}$ give the least and most negative values of $A_{e l}$ for all detectors. Since this tendency is seen globally across all detectors suggest that this can be exploited to determine the correlation between the detectors. The ratio of the separation of $A_{e l}$ between the two models and the total variance of all models gives a correlation of $\mathrm{r} \sim 0.58$. Similar tendencies are seen with the first and third order polynomial models of $A_{b k g}$. We choose the second order polynomial model for $A_{b k g}$ as a reference point, and find the correlation between $\frac{\left|A_{p o l 3}-A_{\text {pol } 2}\right|}{A_{\text {pol } 2}}$ and $\frac{\left|A_{\text {pol } 1}-A_{\text {pol } 2}\right|}{A_{\text {pol } 2}}$, where $A_{p o l 1}, A_{p o l 2}$, and $A_{\text {pol3 }}$ are the elastic asymmetries from the first through third order polynomial models of $A_{b k g}$. A correlation coefficient of $\sim 0.45$ is found based on 14 datapoints (one from each detector).

We estimate that the correlated systematic uncertainty is

$$
\delta A_{\text {el,sys }}^{\text {correlated }}=50 \% \delta A_{\text {el,sys }}
$$

which gives the corresponding point-to-point uncertainty as

$$
\delta A_{e l, s y s}^{p t-p t}=\sqrt{1-50 \%^{2}} \delta A_{e l, s y s}=87 \% \delta A_{e l, s y s}
$$

\section{Detector 15}

The background correction in detector 15 is studied using two different methods. One is a method similar to those for detectors 1-14a; a two-step fitting technique. However, unlike the lower detectors, detector 15 covers a large kinematic range from $0.41<Q^{2}<0.9(\mathrm{GeV} / \mathrm{c})^{2}$, so this detector is separated into three regions of $Q^{2}$. Furthermore, the background yield is no longer interpolated through a sideband fit. It has been seen that the detector 16 yield, which includes only background 
rates, can be scaled to match the detector 15 background yield. The elastic yield can then be determined by subtracting this background yield from the raw measured yield. Once the background yield is determined, two different methods were considered in order to correct for the background asymmetry.

The first method is similar to the method used for detectors 1-14a. But unlike the lower detectors, the elastic asymmetry in detector 15 is modeled with three constants (one for each $Q^{2}$ bin), and a third order polynomial is used for the background asymmetry. Linear and quadratic models were also used for $A_{e l}(t)$, and were seen to give consistent results. Fig. 3.29 shows a sample fit of the raw asymmetry in detector 15 .

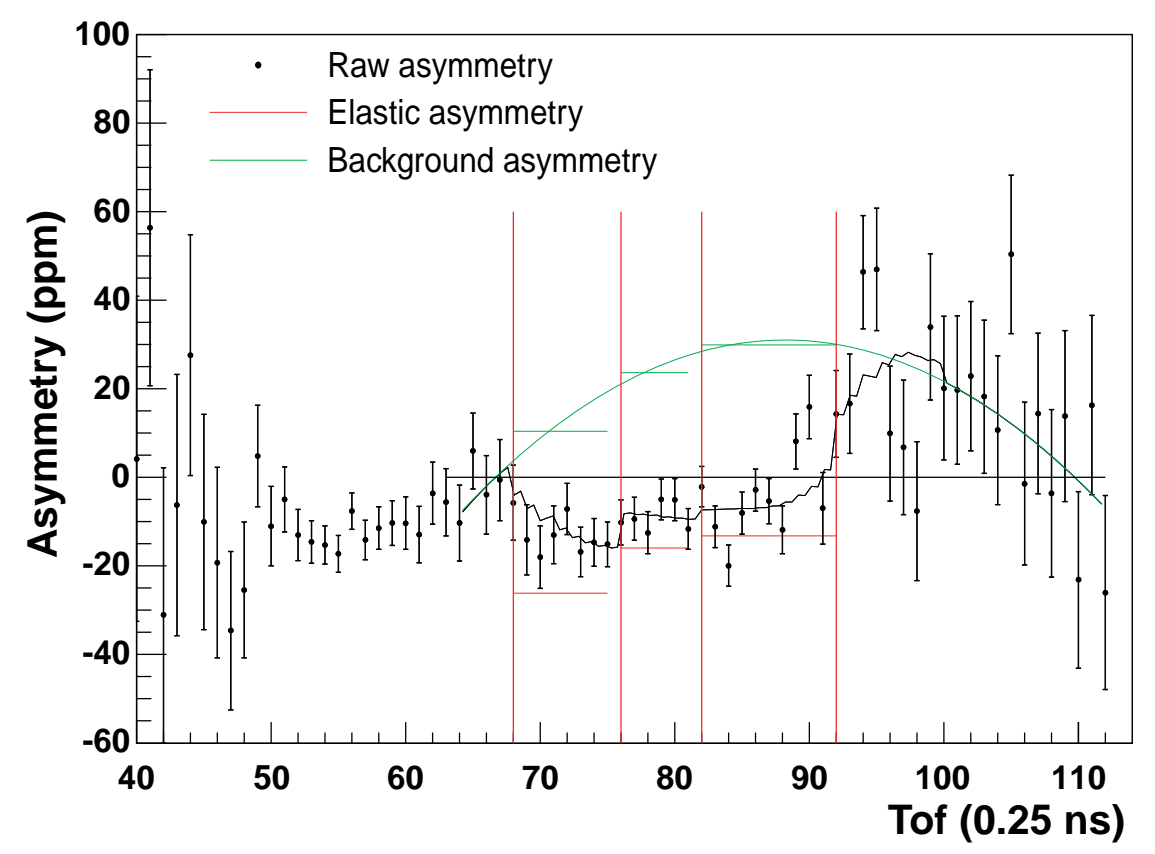

Figure 3.29: Asymmetry fit for octant 2, detector 15. The black points are the measured asymmetries. The red vertical lines show the three $Q^{2}$ bins to which the elastic peak is separated. The green line shows the interpolated background asymmetry from the fit, and the red horizontal lines show the average elastic asymmetry in each of the three $Q^{2}$ bins.

The second method used to correct the detector 15 background is the interpolation across detectors, both for the yield and the asymmetry. We extract the detectors 1 through 14a background asymmetry from the two-step fit, and overlay them as can be seen in Fig. 3.30. What we see is a background asymmetry which tend to increase with detector number. Using this property, 
both the background asymmetry and yield are interpolated across detectors time bin-by-time bin. The interpolated background asymmetry for detector 15 resides between the detectors $14 \mathrm{a}$ and 16 background asymmetries. By contrast, the detector 15 background asymmetry from the two-step fitting technique does not agree well with the other detectors.

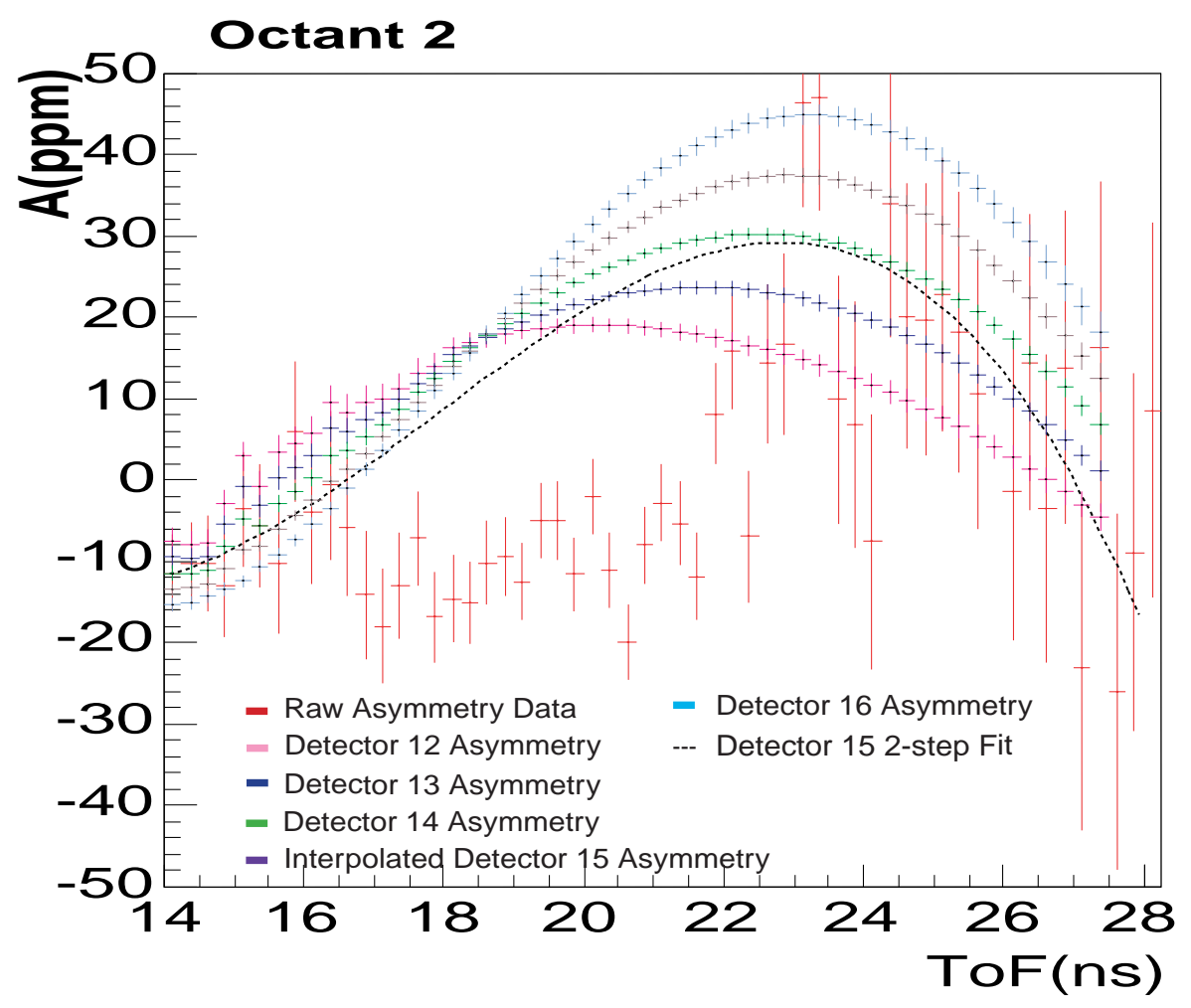

Figure 3.30: Background asymmetries for detectors 12 through 14a, and detector 16. For each time bin, the background asymmetry can be interpolated across these detectors into detector 15 .

Shown in Table 3.5 are the reduced $\chi^{2}$ for the two methods. In the case of the two-step fit, various different functional forms were used to find the optimal fit. We see that the fit is worse for the

\begin{tabular}{|c|c|}
\hline Model & $\chi^{2} / \nu$ \\
\hline two-step $\left(A_{b k g}=\right.$ pol2 $)$ & $423.9 / 407$ \\
\hline Detector-interpolation & $489.2 / 439$ \\
\hline
\end{tabular}

Table 3.5: Reduced $\chi^{2}$ for the two-step fitting technique and the detector-interpolation technique. The $\chi^{2}$ are for all 8 octants combined.

detector-interpolation technique. However, we choose the interpolation technique over the two-step fitting technique for several reasons. First, unlike the lower detectors, the yields themselves (like the asymmetries) are determined by interpolating them across detectors. Also, the interpolation 
technique has additional constraints in that the interpolated background asymmetry must be consistent with what is seen in the other detectors. Furthermore, a study of the octant-dependence of the extracted elastic asymmetry from both methods show a considerably more statistically consistent picture for the interpolation technique as seen in Fig. 3.31. The large positive excursion seen
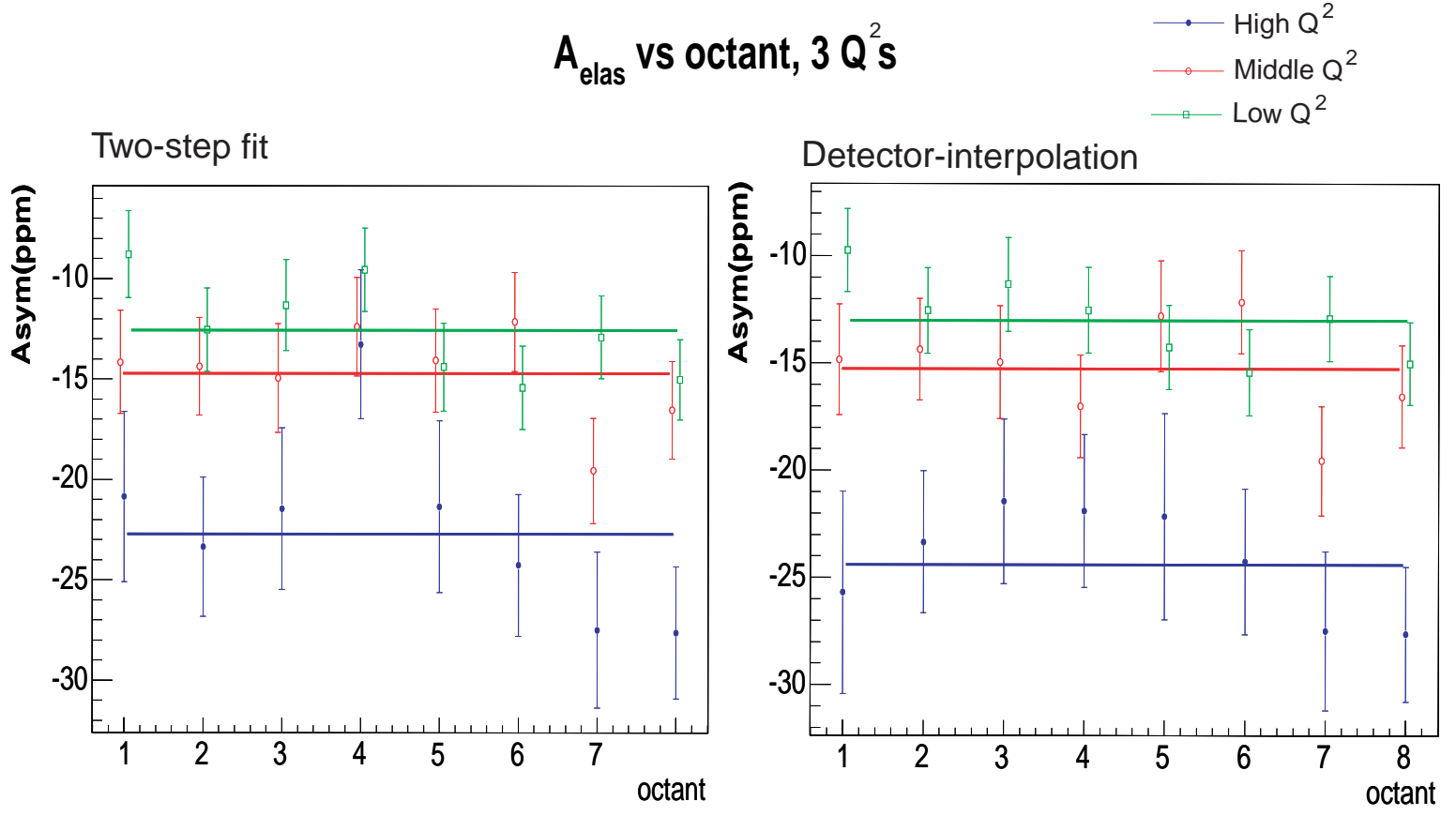

Figure 3.31: Detector 15 elastic asymmetry vs octant. The octant 4 elastic asymmetry shows a large excursion for the two-step technique, particularly for the high $Q^{2}$ points. The interpolation technique shows a more statistically consistent picture.

in octant 4 for the two-step technique is eliminated in the detector-interpolation technique.

For detector 15, Eqn. 3.40 can still be applied to determine the appropriate error that should be assigned. Like the lower detectors, the assignment of the systematic uncertainty in detector 15 involve studies that decouple the correlation between the background yield and asymmetries. As in the lower detectors, the statistical uncertainty is taken from counting statistics. Since the background yield is interpolated across detectors, the upper and lower bounds on the error contribution from the background yield are determined from the yields in detectors 14a and 16

$$
\begin{aligned}
Y_{b k g}^{u p}(15, t) & =0.75 Y_{b k g}(14 a, t)+0.25 Y_{b k g}(16, t) \\
Y_{b k g}^{l o w}(15, t) & =0.25 Y_{b k g}(14 a, t)+0.75 Y_{b k g}(16, t)
\end{aligned}
$$


where $Y_{b k g}(14 a, t)$ and $Y_{b k g}(16, t)$ are the detectors 14a and 16 background yields, and $Y_{b k g}^{u p}(15, t)$ and $Y_{b k g}^{l o w}(15, t)$ are the interpolated upper and lower limits on the detector 15 background yield under the elastic peak. This is similar to using a $\pm \frac{1}{2}$ detector for the uncertainty from the yields. The corresponding systematic uncertainty on the background asymmetry is determined by assigning the sideband-interpolated background asymmetries in detectors $14 \mathrm{a}$ and 16 as the $\pm 1 \sigma$ bound. This error band is enlarged such that it covers the variation when the background asymmetry in detector 15 is shifted in time by $\pm 0.5 \mathrm{~ns}$.

Once the systematic uncertainty on the elastic asymmetry is determined, it can be separated into point-to-point and correlated uncertainties. The elastic asymmetries extracted from the three $Q^{2}$ bins in detector 15 are correlated due to the fact that any particular background correction method uses a specific model for $A_{b k g}(t)$ and $Y_{b k g}(t)$. In order to gain insight into how the two contributions can be separated, the correlated systematic uncertainty is first characterized by an overall scaling variable $S_{i}$

$$
S_{i}=\Sigma_{i} A_{\text {elas }, i}
$$

where $\mathrm{i}=1,2,3 Q^{2}$ bins in detector 15 . The correlation, $R_{i}$, between $A_{\text {elas }, i}$ and $S_{i}$ is then

$$
R_{i}=\frac{\sigma_{A_{e l a s, i}, S_{i}}}{\sigma_{A_{\text {elas }, i}} \sigma_{S_{i}}}
$$

where $\sigma_{A_{\text {elas }, i}, S_{i}}$ is the covariance between the two variables. $R_{i}$ is determined empirically by calculating a set of $A_{\text {elas }, i}$ for a set of $\mathrm{N}$ variations of $A_{b k g}(t)$. From the $\mathrm{N}$ sets of $A_{\text {elas }, i}$, the covariance and hence the correlation terms in Eqn. 3.54 can be calculated. Once this correlation is calculated, the point-to-point and correlated systematic uncertainties can be derived

$$
\begin{gathered}
\sigma_{A_{i, p t-p t}}^{2}=\left(1-R_{i}^{2}\right) \sigma_{A_{i, s y s}}^{2} \\
\sigma_{A_{i, \text { correlated }}}^{2}=R_{i}^{2} \sigma_{A_{i, s y s}}^{2}
\end{gathered}
$$

The models show in the end that the correlation $R_{i}$ is about $50 \%$. 


\section{Detector 14b}

Detector 14b is the elastic peak which resides approximately $4 \mathrm{~ns}$ after the pion peak (see Fig. C.14), and has an average $Q^{2}$ of $0.997(\mathrm{GeV} / \mathrm{c})^{2}$. The peak comes from the fact that the $Q^{2}$ of the elastic protons have a non-linear dependence on the detector number, resulting in two independent elastic peaks in detector 14 .

The determination of the detector $14 \mathrm{~b}$ asymmetry and uncertainty is very similar to those of detectors 1-14a. However, a fifth order polynomial is used to model the background yield, and second to fifth order polynomials are used to model the background asymmetry, and the average value of the elastic asymmetry is taken. Both elastic peaks are fit simultaneously to see their correlations with each other.

In order to determine the contribution of $f_{b}$ to the systematic uncertainty of the elastic asymmetry, $f_{b}(t)$ is scaled randomly with a gaussian, and the two-step fitting procedure is applied to see its effect on $A_{e l}$. Since the choice of $f_{b}(t)$ can affect both $A_{e l}(t)$ and $A_{b k g}(t)$, the variance from this method is equal to

$$
\text { Variance } \left.=\sqrt{(} \delta A_{2}^{2}+\Delta\right)
$$

as defined in Eqn. 3.41. The resulting variance is $7.10 \mathrm{ppm}$.

The effect of the variation of $A_{b k g}$ is studied by putting a \pm 1 detector error band around $A_{b k g}$. The error band is scaled randomly, and the two-step fitting procedure is applied. The resulting variance in $A_{e l}$ gives $\delta A_{3}$ as defined in Eqn. 3.41 for both elastic peaks. We find that $\delta A_{3, l o w Q^{2}}=$ $1.90 \mathrm{ppm}$, and $\delta A_{3, h i g h Q^{2}}=16.74 \mathrm{ppm}$, where $\delta A_{3, \text { low } Q^{2}}$ and $\delta A_{3, h i g h Q^{2}}$ are $\delta A_{3}$ for the low and high $Q^{2}$ elastic peaks, respectively. In order to make a realistic estimate of $\delta A_{3}$ for the high $Q^{2}$ peak, we make a model-dependence study of the lower $Q^{2}$ peak by varying the shape of $A_{b k g}(t)$ from second to fifth order polynomials. We see the variance (or $\delta A_{3}$ ) of the lower $Q^{2}$ elastic asymmetry to be $0.68 \mathrm{ppm}$. Since this method probes the sensitivity of $A_{e l}$ to variations in $A_{b k g}$, it directly gives $\delta A_{3}$. We note that the ratio between the two methods to be $\frac{0.68 p p m}{1.90 p p m} \approx \frac{1}{3}$, and simply scale $\delta A_{3, h i g h Q^{2}}$ using this ratio, giving $\delta A_{3}=5.58 \mathrm{ppm}$ for the high $Q^{2}$ peak. The overall systematic 
uncertainty for this detector is then, $\left.\sqrt{(} \delta A_{2}^{2}+\Delta+\delta A_{3}^{2}\right)=9.03 \mathrm{ppm}$.

Unlike the lower detectors, the uncertainties from either $f_{b}$ or $A_{b k g}$ in detector $14 \mathrm{~b}$ are seen not to be strongly correlated with other detectors or to the 14 a elastic peak. Therefore, the systematic uncertainty from detector 14b background is estimated to be entirely point-to-point.

A summary of the systematic uncertainties for all detectors is presented in Table C.6 in Appendix C.3.

\subsection{Polarization correction}

\section{Longitudinal Polarization}

The longitudinal polarization correction of the asymmetry is, in principle, a simple multiplicative procedure

$$
A_{\text {corr }}=\frac{A_{\text {meas }}}{P}
$$

where $P$ is the polarization, and $A_{\text {corr }}$ and $A_{\text {meas }}$ are the corrected and measured asymmetries. The corresponding contribution to statistical precision in the measurement of the asymmetry due to any dilution of the longitudinal polarization is similar in form

$$
\sigma_{A_{\text {corr }}}^{2}=\frac{\sigma_{A_{\text {meas }}}^{2}}{P^{2}}
$$

where $\sigma_{A_{\text {corr }}}$ and $\sigma_{A_{\text {meas }}}$ are the uncertainties in the corrected and measured asymmetries. However, in addition to the loss of statistical accuracy, the measurement of the longitudinal polarization contributes to the systematic uncertainty assigned to the final parity-violating asymmetry [91]. The Møller systematics can roughly be separated into three categories; target-based systematics, beambased systematics, and systematic effects from the Møller configuration.

\section{Target-based systematics}

In addition to the target polarization discussed in Section 3.9, there are three target-related contributions to the polarization systematic uncertainty. One is the effect of the warping of the Møller target, and another is the heating of the iron target itself. The warping of the target is studied at a solenoidal field of $3 \mathrm{~T}$, and is determined to be about $2^{\circ}$. This gives rise to an uncertainty of 
about $0.37 \%$. The heating of the target due to the beam results in temperature variations of the target across the beam profile. With an asymmetric beam of about $30 \mu \mathrm{m}$ in $\mathrm{x}$ and $100 \mu \mathrm{m}$ in $\mathrm{y}$, the error contribution is about $0.2 \%$ and $0.15 \%$ to the polarization, respectively. A third source of error is the use of heavy iron atoms for the Møller target. Møller scattering off electrons from the internal atomic shells have different kinematics compared to those scattering off electrons from the external shells. The difference in polarimeter acceptance to these two types of events is a source of systematic error, and is called the Levchuk effect. The Levchuk effect is estimated to contribute about $0.3 \%$ to the systematic uncertainty.

\section{Beam-based systematics}

There are several beam-based systematics that can be studied. One is the effect of polarization dilution due to the leakage current. The leakage current is typically about $50 \mathrm{nA}$ out of the $\mathrm{G}^{0}$ beam of $40 \mu \mathrm{A}$, which means the dilution effect is about $0.1 \%$. This leads to a $0.2 \%$ fractional error when the polarization is $75 \%$.

Since the Møller measurements are performed at $2 \mu \mathrm{A}$, we need to determine the uncertainty involved in extrapolating to $40 \mu \mathrm{A}$. Current dependence is investigated by taking Møller measurements at various currents up to $10 \mu \mathrm{A}$. No current dependence is found at the $1 \%$ level, and there are no indications that the beam polarization should change at higher currents. However, a conservative estimate of the uncertainty of $1 \%$ is placed for the low to high current extrapolation.

The uncertainties of the beam position and angle at the Møller target are estimated to be 0.5 $\mathrm{mm}$ and $0.15 \mathrm{mrad}$, respectively. These uncertainties translate into an overall uncertainty in the polarization of about $0.15 \%$ for $\mathrm{x}$ position, $0.03 \%$ for $\mathrm{y}$ position, and $0.04 \%$ for both the $\mathrm{x}$ and $\mathrm{y}$ angles. There are uncertainties in the beam current due to the non-linearity of the gain calibration of the BCMs at low beam current. This non-linearity is directly measured by varying the current, and is deemed negligible. However, an uncertainty of about $10 \mathrm{kHz}$ in the offset of the BCM's gain calibration, which contributes about $0.02 \%$ to the systematic uncertainty.

Another source of systematics is the change in beam tune and accelerator configuration seen over time. Although these changes occurred frequently and resulted in polarization changes on the scale of a few $\%$ at most (see Fig. 3.32), the various Møller measurements are made in such a way 
as to minimize their potential impact (i.e. after extended periods of beam diagnostics or stable LH2 data-taking). Therefore, a systematic uncertainty contribution of about $0.5 \%$ is deemed a conservative estimate.

\section{Møller Configuration}

The systematic effect coming from the Møller polarimeter itself is studied to see how its various components can affect the measurement. The stability of the current inside the Møller magnets as well as the collimators which determine the acceptance of the scattered electrons are considered for systematics. All such effects are seen to contribute $0.1 \%$ or less as seen in Table 3.6. Furthermore, a simulation is performed to determine how well the Møller solenoid fields are known with statistical a uncertainty of about $0.28 \%$.

\section{Summary of Systematic Uncertainties in Polarization}

Table 3.6 shows the various contributions to the Møller systematic uncertainty. The polarization is measured every few days in order to determine its stability over time. Fig. 3.32 shows the measurements over the entire forward angle production run. The final polarization for the forward angle experiment is $73.4 \pm 1.32 \%$. Studies were performed to see whether a difference exists in applying a single average polarization correction to the entire dataset, or applying corrections using polarization measurements that are closest in time to each run [91]. The result of the study shows little difference between the two methods. We therefore used the average polarization to correct the entire dataset.

\section{Transverse Polarization}

The $G^{0}$ beam is not perfectly longitudinally polarized. The main contribution to transverse polarization is probably due to a misaligned Wien filter. Transverse polarization components can cause false asymmetries in parity-violating e-p scattering due to interference between the $1 \gamma$ and $2 \gamma$ exchange amplitudes. The imaginary term in the $2 \gamma$ exchange amplitude creates a vector analyzing power which can cause the cross section to be dependent on the polarization vector and the 


\begin{tabular}{|c|c|c|}
\hline Source & Contribution to Møller uncertainty & Total \\
\hline Target polarization & $0.2 \%$ & \\
\hline Target warping & $0.37 \%$ & \\
\hline Target heating (x/y) & $0.2 / 0.15 \%$ & \\
\hline Levchuk Effect & $0.30 \%$ & $0.55 \%$ \\
\hline Target-based total & $0.2 \%$ & \\
\hline \hline Leakage current & $1 \%$ & \\
\hline 2 to $40 \mu$ A extrapolation & $0.15 / 0.03 \%$ & \\
\hline Beam position (x/y) & $0.04 / 0.04 \%$ & $1.16 \%$ \\
\hline Beam angle (x,y) & $0.5 \%$ & \\
\hline Miscellaneous beam tune change & & \\
\hline Beam-based total & $0.10 \%$ & \\
\hline \hline Q1 magnet current & $0.07 \%$ & \\
\hline Q2 magnet current & $0.02 \%$ & \\
\hline Q2 magnet position & $0.12 \%$ & \\
\hline Multiple Scattering & $0.05 \%$ & \\
\hline Collimator position & $0.1 \%$ & \\
\hline Solenoid field simulation & $0.04 \%$ & \\
\hline Møller electronics deadtime & $0.2 \%$ & \\
\hline Charge asymmetry & $0.28 \%$ & \\
\hline Simulation statistics & & \\
\hline Møller configuration total & & \\
\hline \hline Total Møller systematics & & \\
\hline
\end{tabular}

Table 3.6: Møller systematics.

scattering plane [89]

$$
\sigma(\theta)=\sigma_{0}(\theta)\left[1+A_{n}(\theta) P \cdot \hat{n}\right]
$$

where $A_{n}(\theta)$ is the vector analyzing power, $P$ is the polarization vector, $\theta$ is the scattering angle, and $\sigma(\theta)$ and $\sigma_{0}(\theta)$ are the cross sections with and without the transverse polarization effect. The false asymmetry, $A_{\perp}(\theta)$, caused by this effect is

$$
A_{\perp}(\theta)=A_{n}(\theta)\langle P\rangle
$$

where $\langle P\rangle$ is the average transverse polarization of the beam. Since this polarization has a preferred transverse direction, the asymmetry is also dependent on the azimuthal scattering angle

$$
A_{\perp}(\theta)=A_{n}(\theta) P \sin (\phi)
$$



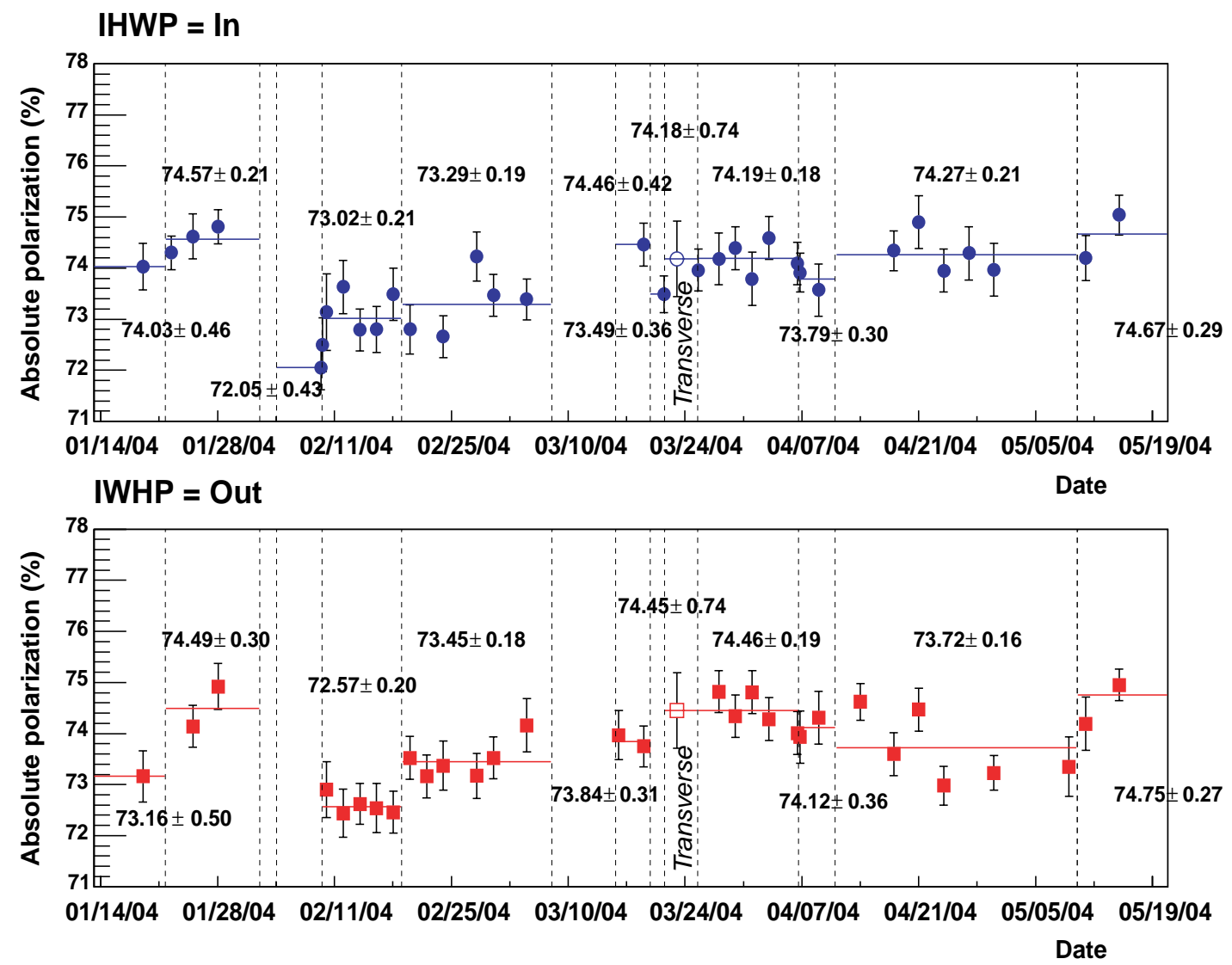

Figure 3.32: Polarization vs run during the $G^{0}$ forward angle measurement. Blue is for IHWP IN, and red is IHWP OUT.

where $\phi$ is the azimuthal angle, and $\delta$ is an overall phase. For parity-violation experiments, the transverse asymmetry alters the measured asymmetry by

$$
A_{\text {meas }}(\theta, \phi)=P \cos \left(\theta_{\text {spin }}\right) A_{P V}(\theta)+P \sin \left(\theta_{\text {spin }}\right) \sin \left(\phi-\phi_{\text {spin }}\right) A_{n}(\theta)
$$

where $A_{\text {meas }}(\theta, \phi)$ is the measured asymmetry, $A_{P V}(\theta)$ is the parity-violating asymmetry, $\theta$ and $\phi$ are the electron scattering angles, and $\theta_{\text {spin }}$ and $\phi_{\text {spin }}$ are the polarization angles.

Therefore, in order to determine the effect of transverse polarization on our measurement, we must know $\theta_{\text {spin }}$, and the vector analyzing power, $A_{n}(\theta)$. We do not need to know $\phi_{\text {spin }}$ due to the azimuthally symmetric nature of the $G^{0}$ spectrometer. As an estimate, we take $\theta_{\text {spin }}$ to be $3^{\circ}$, which is a conservative estimate of how well the Wien angle can be tuned to deliver longitudinally polarized 
beam. Taking the second term in Eqn. 3.64, this gives a false asymmetry that is approximately

$$
A_{\text {trans }}(\theta, \phi) \approx 0.05 \cdot P \sin \left(\phi-\phi_{\text {spin }}\right) A_{n}(\theta)
$$

Dedicated transverse polarization runs were taken in order to determine the vector analyzing power, $A_{n}(\theta)$ [89]. The Wien filter is adjusted in order to orient the beam spin in the transverse direction. The Møller polarimeter is only sensitive to the longitudinal component of beam polarization, so the Wien angle is altered until the measured longitudinal polarization is zero. The optimal Wien angle was determined to be $-85^{\circ}$, which corresponds to a $90^{\circ}$ spin direction in Hall C. Fig. 3.33 shows the asymmetry measured due to transverse beam polarization as a function of octant. Although the asymmetries shown in Fig. 3.33 are not corrected for the various systematic effects such as background or leakage, these sources of false asymmetry typically do not carry systematic azimuthal dependence ${ }^{1}$. The polarization angle is now $90^{\circ}$ (transverse), so the relation describing the curves in Fig. 3.33 is Eqn. 3.64 with $\theta_{\text {spin }}=90^{\circ}$, or

$$
A_{\text {false }}(\theta, \phi)=P \sin \left(\phi-\phi_{\text {spin }}\right) A_{n}(\theta)
$$

where $P \cdot A_{n}(\theta)$ is the approximately $2 \mathrm{ppm}$ amplitude seen in Fig. 3.33. Combining this result with Eqn. 3.65, we get a false asymmetry which azimuthally oscillates with a $0.1 \mathrm{ppm}$ amplitude. However, this is clearly an overestimate due to the fact that the asymmetry we measure will, in the end, be averaged over the octants reducing the overall effect by at least an order of magnitude. Furthermore, the degree to which the polarization direction may not be longitudinal by as much as $3^{\circ}$ is conservative. We therefore estimate that the false asymmetry contribution from transverse polarization to be of order $0.01 \mathrm{ppm}$, which is negligible.

\subsection{Radiative corrections}

This section will discuss the effect of ordinary electromagnetic radiative corrections [90]. These radiative corrections (RC) are common, unavoidable features of elastic electron scattering. Radiation

\footnotetext{
${ }^{1}$ The linear regression corrections, which do carry azimuthal dependence are about two orders of magnitude smaller than the asymmetry from the vector analyzing power of the transversely polarized beam.
} 

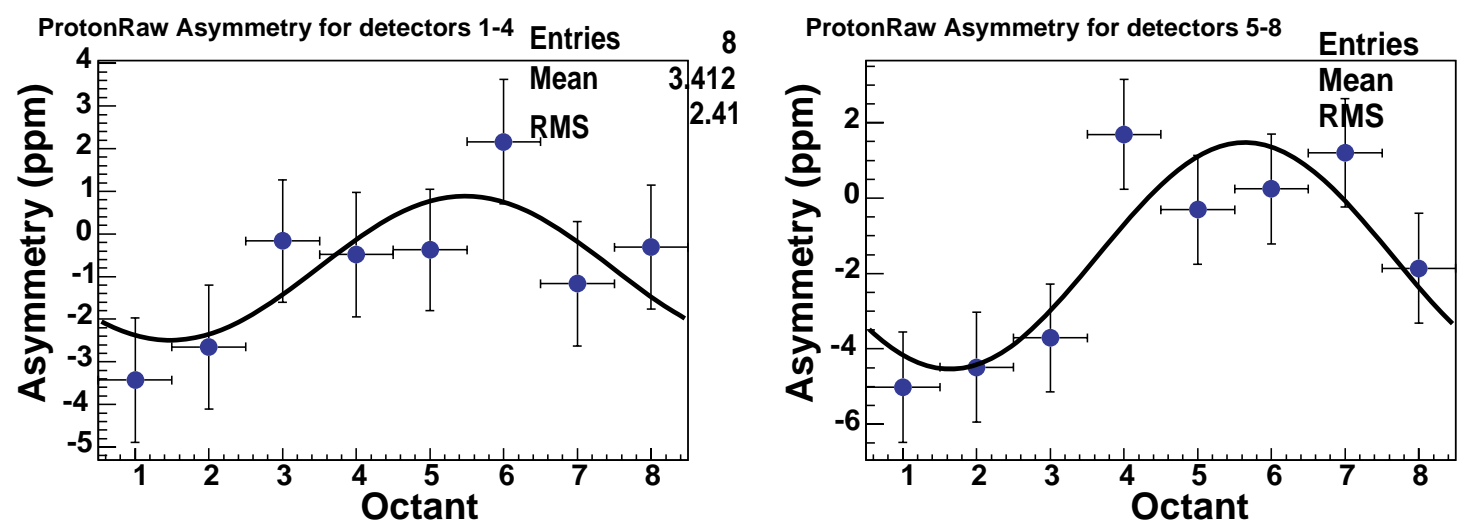

8
3.142

2.265
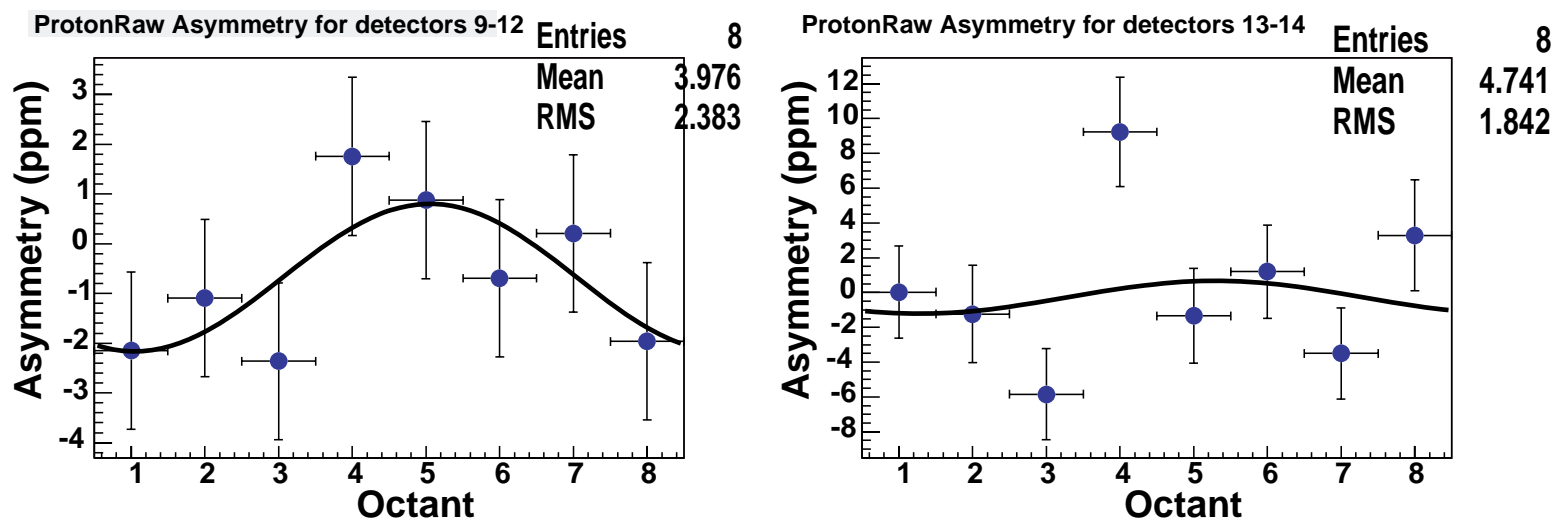

Figure 3.33: Transverse asymmetry measurement. Asymmetries are averaged by four detectors (except detectors 13 and 14, which are averaged together) in order to improve the statistical uncertainty. One can see the sinusoidal dependence of the asymmetry as expected.

of photons induces energy loss in the incident and scattered electrons, and affect the kinematics of the scattered particle. Therefore, the radiative corrections must correct for changes in proton rate and asymmetry, as well as the $Q^{2}$. G0GEANT is used to determine the appropriate corrections to apply.

There are four types of RC to consider. One is energy loss of the incident electron due to target ionization. The energy loss from G0GEANT shows an average energy loss at target midpoint of about 5 to $15 \mathrm{MeV}$ at target midpoint. Another source of energy loss is the emission of bremsstrahlung photons by the incident electron in nuclear interactions. The emission of the 
photon reduces the energy, and the result is a broad energy spectrum. The total average energy loss is about $40 \mathrm{MeV}$, but can reach as high as $3 \mathrm{GeV}$ (the total beam energy).

The two remaining $\mathrm{RC}$ to consider are the virtual and real internal radiative corrections which involve photon emission associated with the actual electron scattering vertex. Virtual internal corrections refer to the emission of a photon which is re-absorbed so that the final state is similar to that from elastic scattering. Although this does not modify the proton kinematics, it can affect the momentum transfer as well as the polarization of the electron at the scattering vertex. Fig. 3.34 shows the various scattering amplitudes that are calculated in addition to the tree level (Born) term. Real internal RC involve the emission of a real photon such that there is a three-body final state

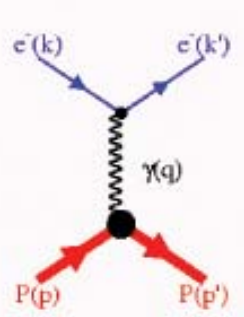

(a0)

Born

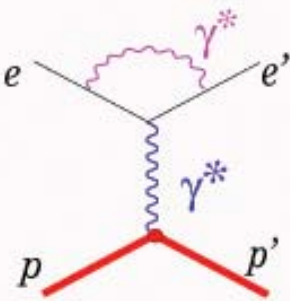

(a)

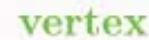

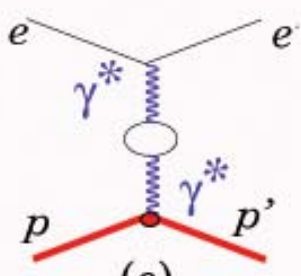

(c)

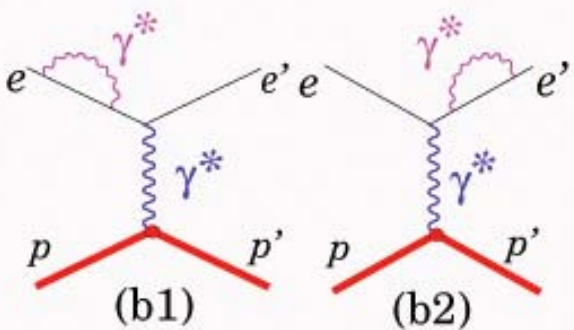

self energy

Figure 3.34: Amplitudes for virtual internal radiative corrections. The electrons emit and re-absorb virtual photons [90].

$(\mathrm{e}, \mathrm{p}, \gamma)$. This effect can again modify the momentum transfer and the electron polarization at the scattering vertex (in the case where the photon is emitted prior to the scattering). We neglect the real photon emission from the proton. The Feynman diagram showing this effect can be seen in Fig. 3.35. 


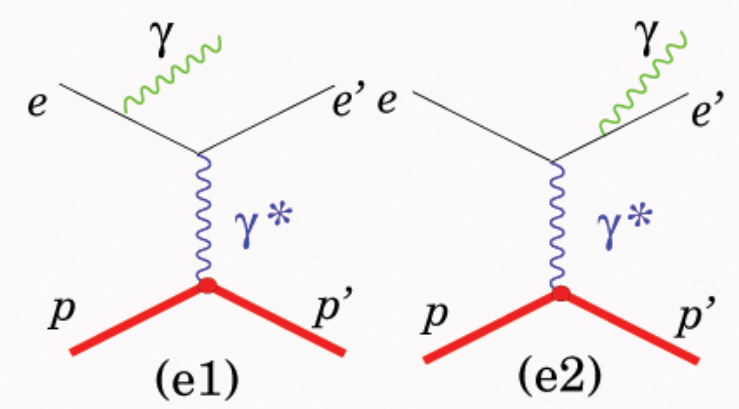

Figure 3.35: Amplitudes for real internal radiative corrections. The emission of a real photon results in a three-body final state which changes the kinematics of the recoil proton [90].

The corrections are simulated using G0GEANT. The incident energy of the electron is varied in order to see the overall effect of energy loss on the elastic rate. Although, in principle, the beam can lose all its energy through radiation, the probability that it loses more than $500 \mathrm{MeV}$ is only about $4 \%$. About $60 \%$ of the electrons lose $1 \mathrm{keV}$ or less. Therefore, the incident beam energy for the simulation is varied between 2.5 and $3.0 \mathrm{GeV}$. The simulation is run twice; with and without the RC. The overall effect is that correction decreases the size of the asymmetry.

The resulting correction to the asymmetry is put in the form of an overall multiplicative factor. Fig. 3.36 shows the effect of $\mathrm{RC}$ on the asymmetries. The plot shows that RC affects the asymmetry by at most $3 \%$.

The uncertainty in this correction is based on the conservative estimate that the elastic cuts in the tof spectra have a $10 \%$ uncertainty. We therefore calculate the correction factors for cuts that are $5 \%$ larger and smaller than the standard elastic cut. The resulting uncertainties are simulated to be at most $0.4 \%$ of the asymmetry.

\subsection{Summary}

The various corrections to the asymmetry detailed in this section are applied in a particular order such that double-corrections do not occur. The first correction is that for deadtime and is applied at the time the raw data are initially replayed. The deadtime corrections are applied first in 


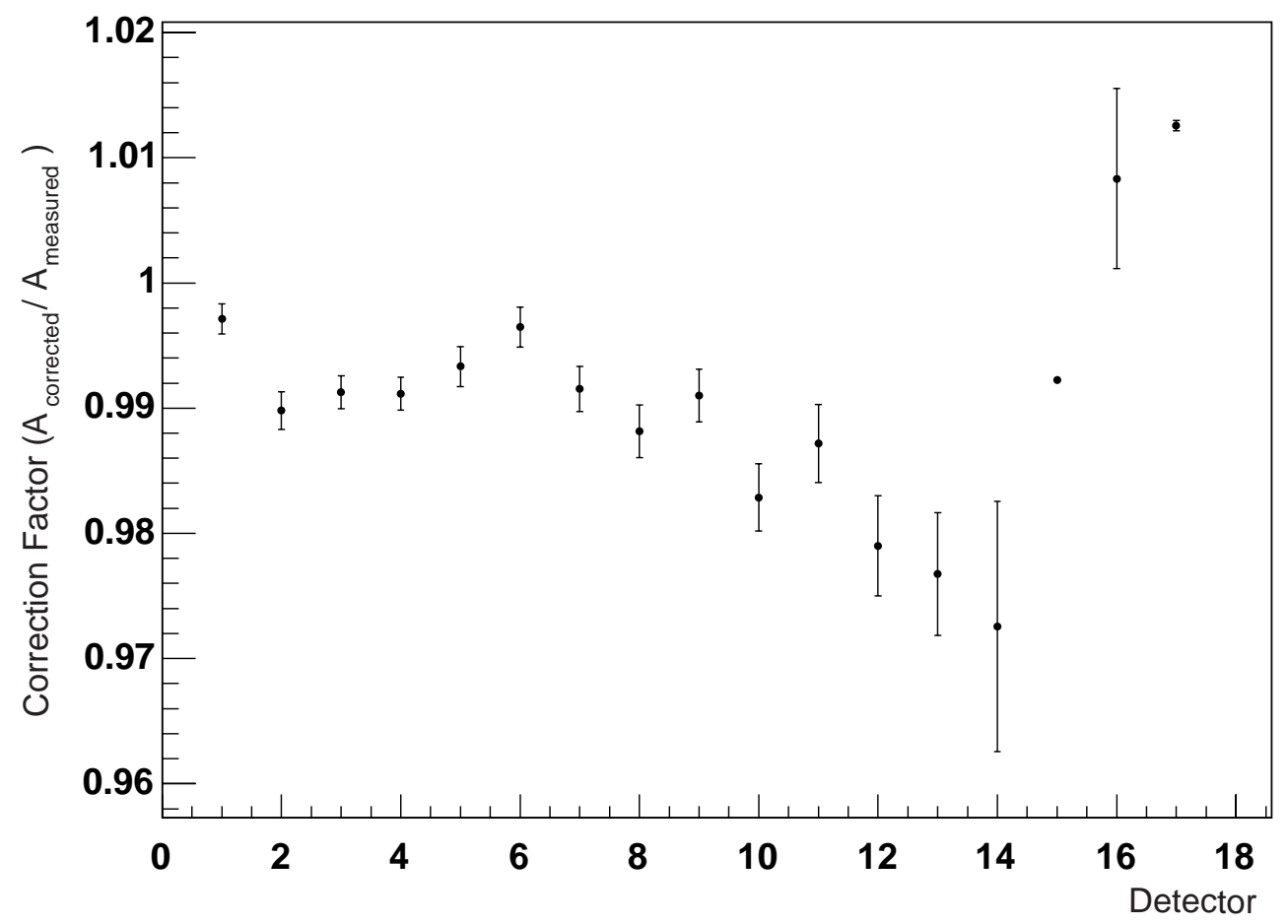

Figure 3.36: Effect of radiative corrections to the measured asymmetry. The simulation is analyzed with a \pm 2 ns cut defining the elastic peak, and using both the NA and FR tof resolution.

order to reduce the effect of charge asymmetry on the data before linear regression corrections are applied. The corrections are then applied in the order: beam leakage, background, residual deadtime, linear regression, and polarization. These corrections are made on an FPD-by-FPD basis. Once all the corrections are applied, the asymmetries are averaged over all octants, the blinding factor is removed, and the final physics asymmetry is revealed. A table of correction factors for each systematic and their uncertainties can be seen in Tables C.5 and C.6, respectively, in Appendix C.3. The systematics are clearly dominated by the background correction, particularly in the higher detectors. Table 3.7 and Fig. 3.37 show the measured asymmetries (not corrected for background) and final asymmetries for each detector and their $Q^{2}$. The blinding factor is removed from the physics asymmetry and, retroactively, from the measured asymmetry as well. The blinding factor, at the end of the analysis, was determined to be a multiplicative factor of 0.8056 . 


\begin{tabular}{|c|c|c|c|c|c|c|}
\hline Detector & $Q^{2}$ & $A_{\text {meas }}$ & $A_{\text {phys }}$ & $\sigma_{\text {stat }}$ & $\sigma_{\text {pt-pt }}$ & $\sigma_{\text {correlated }}$ \\
\hline 1 & 0.122 & -1.38 & -1.51 & 0.44 & 0.22 & 0.18 \\
\hline 2 & 0.128 & -1.07 & -0.97 & 0.41 & 0.20 & 0.17 \\
\hline 3 & 0.136 & -1.34 & -1.30 & 0.42 & 0.17 & 0.17 \\
\hline 4 & 0.144 & -2.67 & -2.71 & 0.43 & 0.18 & 0.18 \\
\hline 5 & 0.153 & -2.46 & -2.22 & 0.43 & 0.28 & 0.21 \\
\hline 6 & 0.164 & -3.13 & -2.88 & 0.43 & 0.32 & 0.23 \\
\hline 7 & 0.177 & -4.47 & -3.95 & 0.43 & 0.25 & 0.20 \\
\hline 8 & 0.192 & -5.01 & -3.85 & 0.48 & 0.22 & 0.19 \\
\hline 9 & 0.210 & -5.73 & -4.68 & 0.47 & 0.26 & 0.21 \\
\hline 10 & 0.232 & -6.08 & -5.27 & 0.51 & 0.30 & 0.23 \\
\hline 11 & 0.262 & -5.55 & -5.26 & 0.52 & 0.11 & 0.17 \\
\hline 12 & 0.299 & -5.40 & -7.72 & 0.60 & 0.53 & 0.35 \\
\hline 13 & 0.344 & -3.65 & -8.40 & 0.68 & 0.85 & 0.52 \\
\hline $14 \mathrm{a}$ & 0.410 & -1.70 & -10.25 & 0.67 & 0.89 & 0.55 \\
\hline $15 \mathrm{a}$ & 0.511 & -5.80 & -16.81 & 0.89 & 1.48 & 1.50 \\
\hline $15 \mathrm{~b}$ & 0.631 & -9.74 & -19.96 & 1.11 & 1.28 & 1.31 \\
\hline $15 \mathrm{c}$ & 0.788 & -12.66 & -30.8 & 1.9 & 2.6 & 2.59 \\
\hline $14 \mathrm{~b}$ & 0.997 & 4.21 & -37.9 & 7.2 & 9.0 & 0.52 \\
\hline
\end{tabular}

Table 3.7: Measured and corrected asymmetries. The blinding factor is taken off of both sets of asymmetries.

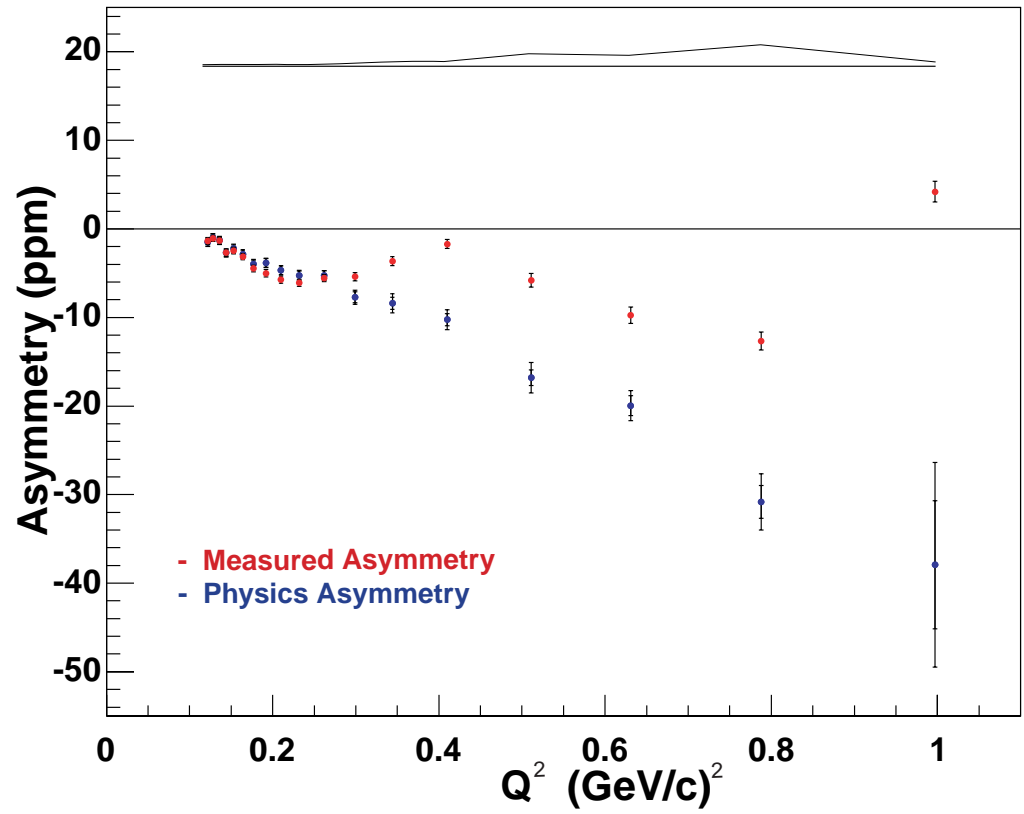

Figure 3.37: Measured and corrected asymmetries. The physics asymmetry show the statistical (inner) and statistical + point-to-point systematic (outer) error bars. The errors on the raw asymmetry are calculated from the widths of the raw asymmetry distribution over all runs. The band at the top shows the correlated uncertainties for each $\mathrm{Q}^{2}$ point. 


\section{Chapter 4}

\section{Results and Conclusions}

\section{Results}

The expression for the strange quark form factors of the proton can be directly derived from Eqn. 1.43

$$
G_{E}^{s}+\eta G_{M}^{s}=\frac{4 \sqrt{2} \pi \alpha\left(\eta\left(G_{E}^{\gamma}\right)^{2}+\tau\left(G_{M}^{\gamma}\right)^{2}\right)}{G_{F} Q^{2} \eta G_{E}^{\gamma}}\left(A_{p h y s}-A_{N V S}\right)
$$

where $\eta=\frac{\tau G_{M}^{p}}{\epsilon G_{E}^{p}}, A_{\text {phys }}$ is the final physics asymmetry measured by the $G^{0}$ forward angle measurement, and $A_{N V S}$ is the theoretical asymmetry assuming no vector strange quark contribution to proton structure.

The proton and neutron electromagnetic form factors $\left(G_{E}^{\gamma, p}, G_{M}^{\gamma, p}, G_{E}^{\gamma, n}, G_{M}^{\gamma, n}\right)$ are parameterizations from work by J.J.Kelly [92]. This parameterization of the electromagnetic form factors makes use of data taken from both the recent recoil polarization measurements as well as those from Rosenbluth separation. However, for $Q^{2}>1(\mathrm{GeV} / \mathrm{c})^{2}$, the Rosenbluth data are omitted due to discrepancies in results between the two methods. This high $Q^{2}$ region is beyond the kinematic range of $G^{0}$. Recent studies have suggested that the difference arises from two-photon exchange between the beam and the target, which manifests itself as a higher order correction which is not included in the usual set of radiative corrections [93]. Resolving this discrepancy is a work in progress; however, it appears that two-photon corrections have a much smaller effect on the polarization transfer data.

Table 4.1 and Fig. 4.1 show the $Q^{2}$ dependence of $G_{E}^{s}+\eta G_{M}^{s}$. The results from the HAPPEX experiment are also shown in the figure, and are in excellent agreement with the nearest $G^{0}$ datapoints. As seen in Eqn. 4.1, the linear combination of $G_{E}^{s}+\eta G_{M}^{s}$ is proportional to the difference 


\begin{tabular}{|c|c|c|c|c|c|c|c|}
\hline Detector & $Q^{2}$ & $\eta$ & $G_{E}^{s}+\eta G_{M}^{s}$ & $\sigma_{\text {stat }}$ & $\sigma_{\text {sys,pt-pt }}$ & $\sigma_{\text {sys,correlated }}$ & $\sigma_{\text {sys,model }}$ \\
\hline 1 & 0.122 & 0.098 & 0.037 & 0.036 & 0.019 & 0.015 & 0.003 \\
\hline 2 & 0.128 & 0.103 & 0.090 & 0.032 & 0.016 & 0.014 & 0.003 \\
\hline 3 & 0.136 & 0.109 & 0.074 & 0.032 & 0.013 & 0.013 & 0.003 \\
\hline 4 & 0.144 & 0.116 & -0.014 & 0.030 & 0.013 & 0.012 & 0.003 \\
\hline 5 & 0.153 & 0.123 & 0.034 & 0.028 & 0.019 & 0.014 & 0.003 \\
\hline 6 & 0.164 & 0.132 & 0.010 & 0.026 & 0.020 & 0.014 & 0.004 \\
\hline 7 & 0.177 & 0.143 & -0.028 & 0.024 & 0.014 & 0.011 & 0.004 \\
\hline 8 & 0.192 & 0.156 & 0.003 & 0.025 & 0.011 & 0.010 & 0.004 \\
\hline 9 & 0.210 & 0.170 & -0.010 & 0.022 & 0.012 & 0.010 & 0.004 \\
\hline 10 & 0.232 & 0.189 & -0.002 & 0.021 & 0.012 & 0.010 & 0.004 \\
\hline 11 & 0.262 & 0.214 & 0.038 & 0.019 & 0.004 & 0.006 & 0.005 \\
\hline 12 & 0.299 & 0.245 & 0.002 & 0.018 & 0.016 & 0.011 & 0.005 \\
\hline 13 & 0.344 & 0.283 & 0.033 & 0.018 & 0.022 & 0.014 & 0.005 \\
\hline $14 \mathrm{a}$ & 0.410 & 0.341 & 0.053 & 0.014 & 0.019 & 0.012 & 0.006 \\
\hline $15 \mathrm{a}$ & 0.511 & 0.431 & 0.019 & 0.014 & 0.024 & 0.024 & 0.006 \\
\hline $15 \mathrm{~b}$ & 0.631 & 0.543 & 0.060 & 0.014 & 0.016 & 0.016 & 0.007 \\
\hline $15 \mathrm{c}$ & 0.788 & 0.700 & 0.036 & 0.018 & 0.024 & 0.025 & 0.008 \\
\hline $14 \mathrm{~b}$ & 0.997 & 0.932 & 0.076 & 0.052 & 0.064 & 0.004 & 0.008 \\
\hline
\end{tabular}

Table 4.1: Final results of the $G^{0}$ forward angle measurement.

between $A_{\text {phys }}$ and $A_{N V S}$. Therefore, if the measured asymmetry were to show no strange quark content, the data would lie at $G_{E}^{s}+\eta G_{M}^{s}=0$. The green dashed and magenta dotted lines toward the bottom of the plot show how the $G_{E}^{s}+\eta G_{M}^{s}=0$ line would change with respect to the data when the Friedrich and Walcher (FW) [94] and Arrington [93] parameterizations, respectively, are used to calculate the electromagnetic form factors (e.g. for the FW parameterization, the value of $G_{E}^{s}+\eta G_{M}^{s}$ at $Q^{2}=0.63(G e V / c)^{2}$ increases from 0.059 to 0.072$)$.

To characterize the result with a single number, we tested the hypothesis that $G_{E}^{s}+\eta G_{M}^{s}=$ 0 by generating randomized data sets with this constraint, distributed according to our statistical and systematic uncertainties. The fraction of these with $\chi^{2}$ larger than that of our data set was $11 \%$. We therefore conclude that our results exclude the possibility of the no-strange hypothesis of the proton with $89 \%$ confidence.

At low $Q^{2}$ near $0.1(G e V / c)^{2}$, the results are consistent with previous experiments that $G_{M}^{s} \approx$ 0.5, with $\eta=0.1$ from Table 4.1, making $\eta G_{M}^{s}$ about 0.05 . Combining the results with previous experiments at $Q^{2}=0.1(\mathrm{GeV} / \mathrm{c})^{2}$ as seen in Fig. 4.2, we get $G_{M}^{s}\left(Q^{2}=0.1\right)=0.62 \pm 0.31$. Therefore, at this $Q^{2}$, we do see strange quarks contributing to the magnetic structure of the 


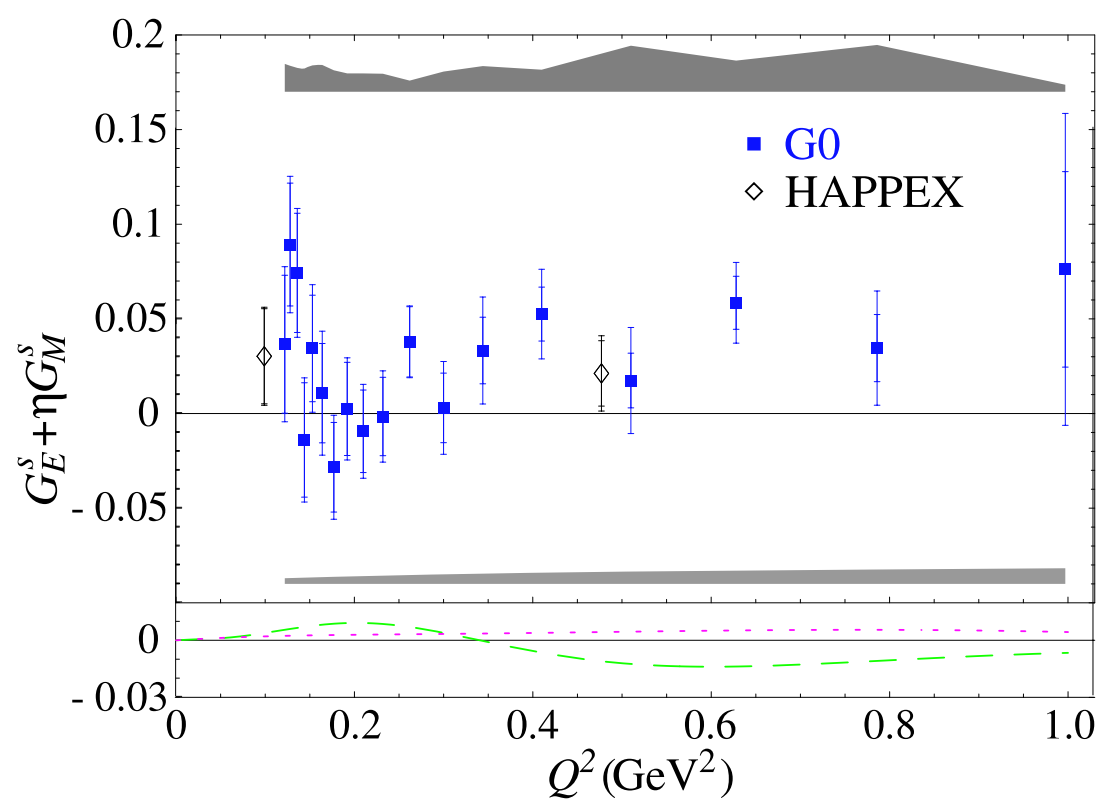

Figure 4.1: $G_{E}^{s}+\eta G_{M}^{s}$ vs $Q^{2}$. The data points have inner (statistical) and outer (statistical + point to point systematic) error bars. The gray bands indicate correlated (top band) and model (lower band) systematic uncertainties. The lines in the lower panel correspond to different electromagnetic nucleon form factor models.

proton at a $2 \sigma$ level.

But, perhaps more intriguing, is the $Q^{2}$ dependence seen in the results. The kinematic factor, $\eta$, increases approximately linearly with $Q^{2}$. The initial rise of this linear combination from $Q^{2}=0$ to about $Q^{2}=0.15$ is driven by this kinematic factor and the fact that $G_{M}^{s}>0$ as seen at $Q^{2}=0.1$ $(G e V / c)^{2}$. However, $G_{E}^{s}+\eta G_{M}^{s}$ appears to show a drop with a minimum around $0.23(G e V / c)^{2}$ despite the fact that $\eta$ continues to increase. This suggests that $G_{E}^{s}$ may itself have a significant $Q^{2}$ dependence, turning negative near this kinematic point. Although it is, in principle, possible that $\eta G_{M}^{s}$ undergoes a rapid change in the negative direction in this intermediate range, combining the $G^{0}$ results with that from PVA4 indicate that it is more likely that $G_{E}^{s}$ is negative (Fig. 4.3). Phenomenologically, a negative "bump" in $G_{E}^{s}$ suggests that there is spatial separation between the $s$ and $\bar{s}$ distributions, with the $\bar{s}$ quarks closer to the center of the proton and the $s$ distributed around this core. Additional backward angle measurements will further improve our knowledge at this $Q^{2}$.

At $Q^{2}=0.477(\mathrm{GeV} / \mathrm{c})^{2}$ (Fig. 4.4), the two existing measurements show excellent agreement, 
but more measurements are needed to separate $G_{E}^{s}$ and $G_{M}^{s}$. At higher $Q^{2}$, above about 0.5 $(G e V / c)^{2}$, there is a significant trend favoring a positive value for $G_{E}^{s}+\eta G_{M}^{s}$. However, the behavior of either strange form factors by themselves cannot be determined or even deduced at present, due to the fact that no measurements have been made at such high $Q^{2}$ other than those by $G^{0}$.

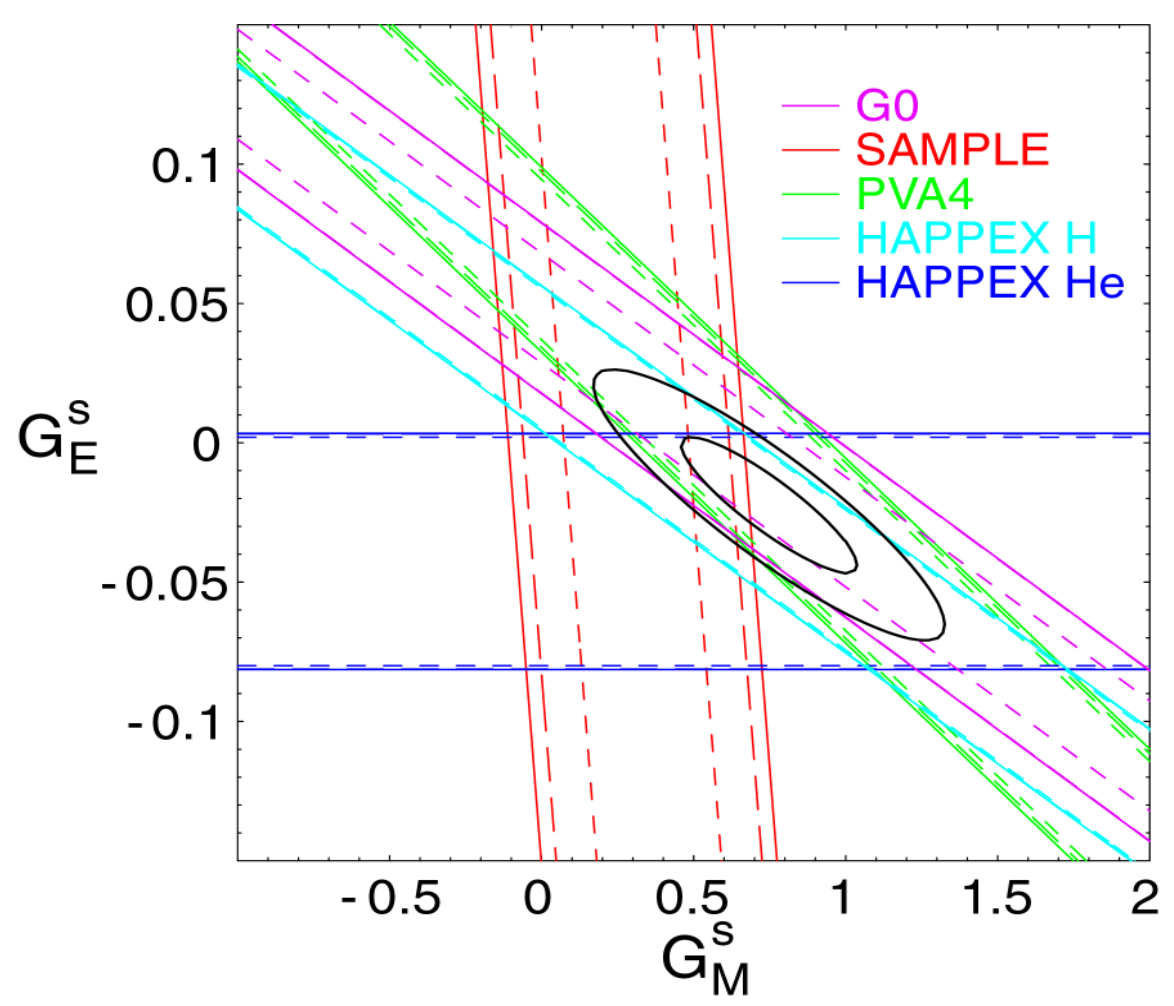

Figure 4.2: Band plot of all world data at $Q^{2}=0.1(\mathrm{GeV} / \mathrm{c})^{2}$. Combining experiments with different angular kinematics but the same $Q^{2}$ allows for the separation of $G_{E}^{s}$ and $G_{M}^{s}$. The ellipses are $68.3 \%$ (inner) and $95.5 \%$ (outer) confidence intervals.

\section{Outlook}

Future experiments, combined with the present $G^{0}$ results, will give further insight into the behavior of $G_{E}^{s}$ and $G_{M}^{s}$. $G^{0}$ backward angle measurements as well as experiments scheduled to take place at MAMI (Mainz) will directly separate the two form factors in the regions of interest in Fig.4.1. The HAPPExII experiment will add tighter constraints on $G_{E}^{s}$ and $G_{M}^{s}$ at $Q^{2}=0.1(G e V / c)^{2}$.

The $G^{0}$ backward angle measurement involves physically rotating the spectrometer $180^{\circ}$ around the beamline such that the FPDs are located upstream of the target and the magnet. Unlike in 


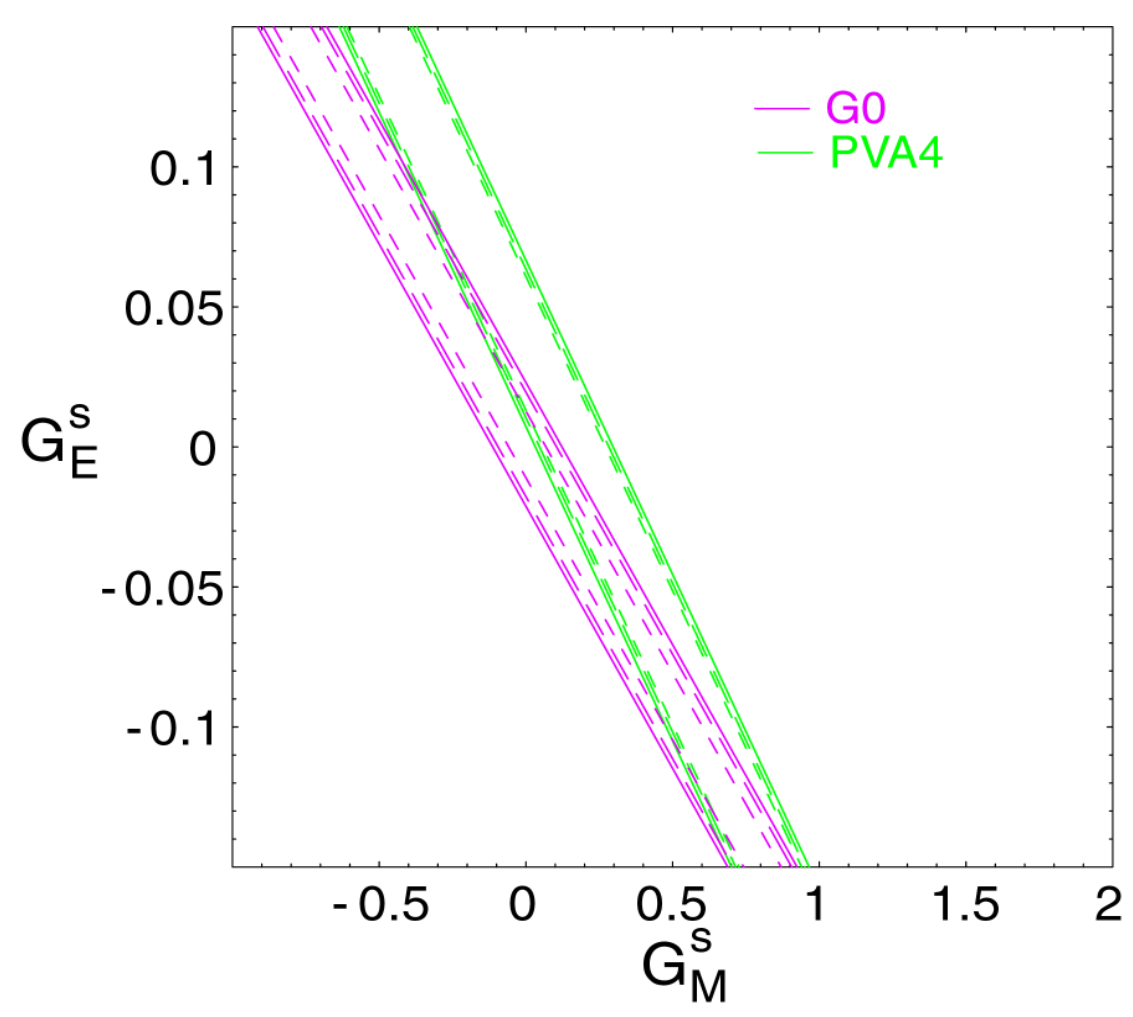

Figure 4.3: Band plot of all world data at $Q^{2}=0.23(\mathrm{GeV} / \mathrm{c})^{2}$. There are less measurements taken at this $Q^{2}$, and the kinematics are relatively close between the existing two measurements. Although the overlap region of the two bands seem to favor a negative $G_{E}^{s}$, a backward angle measurement at this $Q^{2}$ would allow for a much improved separation between $G_{E}^{s}$ and $G_{M}^{s}$.

the forward angle mode, the backward angle mode measures back-scattered electrons in parityviolating electron-proton and quasi-elastic electron-deuteron scattering. A set of nine Cryostat Exit Detectors (CED) are placed between the FPDs and the magnet for each octant. Particles are no longer identified through tof due to the fact that the fast moving electrons are indistinguishable in time from pions and gammas. Identification is made by observing coincidence hits between FPDs and CEDs, and creating a $9 \times 16$ CED-FPD matrix detector/electronics system. With this system, the particle momenta and angles are measured allowing for separation of elastic and inelastic electrons. Furthermore, Cerenkov detectors are placed between the CEDs and FPDs for the purpose of background (mostly pions) rejection. Although the backward angle scheme allows only one $Q^{2}$ measurement at a time, it has the advantage that it will be using the nominal $499 \mathrm{MHz}$ JLab beam structure. This provides an advantage compared to the forward angle measurement in that the experiment will not be susceptible to leakage from other halls, since the beam structures between 


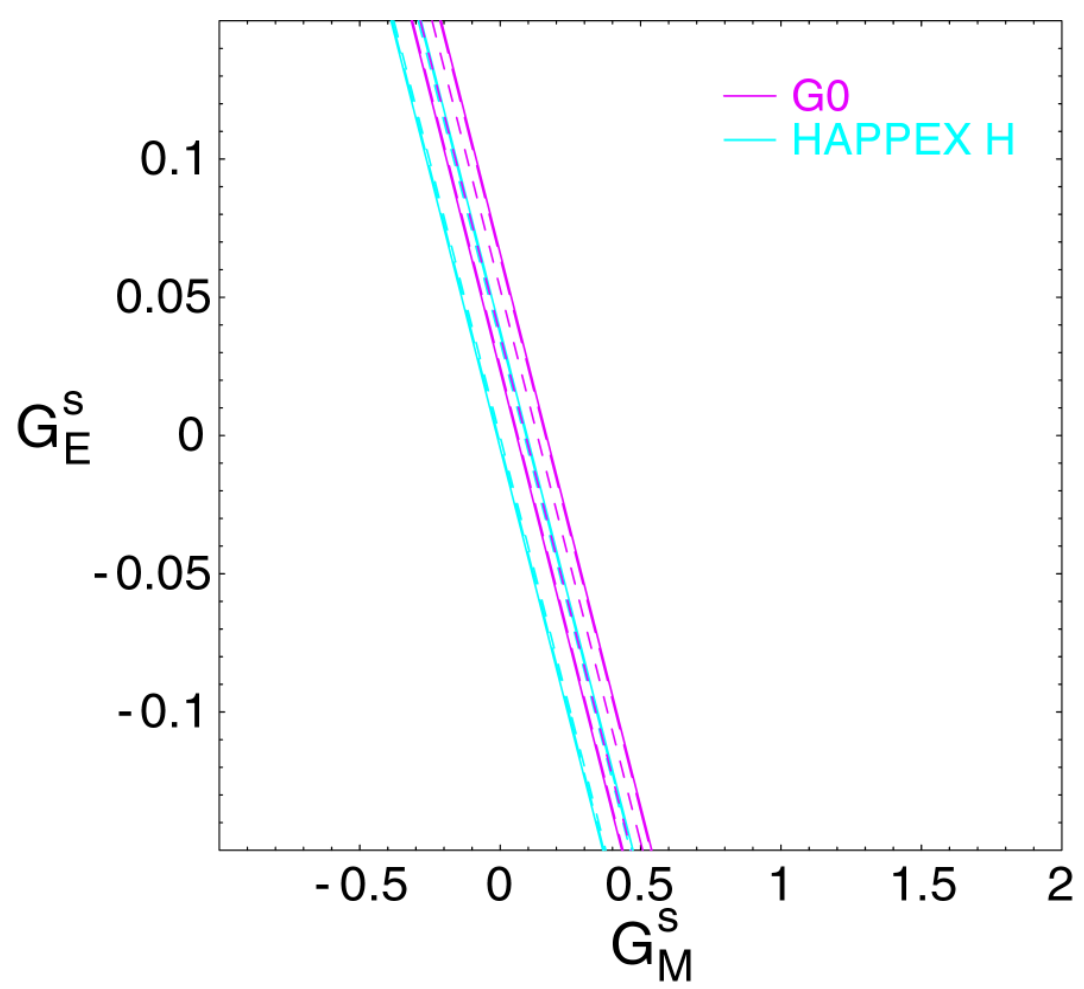

Figure 4.4: Band plot of all world data at $Q^{2}=0.477(\mathrm{GeV} / \mathrm{c})^{2}$. The kinematics for the two experiments are very close, making the two bands near-parallel. They are in good agreement with each other. A backward angle measurement is needed in order to separate $G_{E}^{s}$ and $G_{M}^{s}$.

the three halls will be identical. The backward angle experiment is currently scheduled to run starting spring of 2006 at $Q^{2}=0.63(\mathrm{GeV} / \mathrm{c})^{2}$, and additional measurements at $Q^{2}=0.23(\mathrm{GeV} / \mathrm{c})^{2}$ are expected over the summer.

The PVA4 collaboration at Mainz, which has concluded their forward angle measurements at $Q^{2}=0.108$ and $0.230(\mathrm{GeV} / \mathrm{c})^{2}$ (Fig. 4.2 and 4.3), plan to rotate their calorimeter by $180^{\circ}$ and conduct backward angle measurements at $Q^{2}=0.23$ and $0.477(\mathrm{GeV} / \mathrm{c})^{2}$. This, in addition to the $G^{0}$ backward angle run, would further facilitate the separation of the strange form factors.

The HAPPEx collaboration is currently conducting the second forward angle measurement at $0.1(\mathrm{GeV} / \mathrm{c})^{2}$. The experiment includes two measurements, with a $3.2 \mathrm{GeV}$ polarized electron beam incident on a ${ }^{4} \mathrm{He}$ and a hydrogen target, and an elastic electron scattering angle of $6^{o}$. The measurement with the hydrogen target measures the linear combination of $G_{E}^{s}+\eta G_{M}^{s}$ as is the case in the $G^{0}$ forward angle measurement. However, the asymmetry measured with a ${ }^{4} \mathrm{He}$ target has the advantage that it is sensitive only to $G_{E}^{s}$. This comes from the fact that elastic electron scattering 
with ${ }^{4} \mathrm{He}$ is an isoscalar $0^{+}$to $0^{+}$transition. The asymmetry, therefore, has no contributions from magnetic or axial-vector terms

$$
A_{4}^{P V}=-\frac{A_{0}}{2}\left(2 \sin ^{2} \theta_{w}+\frac{G_{E}^{s}}{G_{E}^{p \gamma}+G_{E}^{n \gamma}}\right)
$$

where $A_{4}^{P V} \mathrm{He}$ is the asymmetry measured from the ${ }^{4} \mathrm{He}$, and $A_{0}=\frac{G_{F} M_{p}^{2}}{\sqrt{2} \pi \alpha}$. A fraction of the statistics has been accumulated during a summer 2004 run, and the results have been published. As can be seen on Fig. 4.2, this adds a significant constraint to the determination of $G_{E}^{s}$. The remaining statistics have been accumulated during the fall/winter 2005 run. The measurements that are presently being analyzed should reduce the uncertainties in the two HAPPEx bands on Fig. 4.2 by a factor of 2-3.

\section{Conclusion}

Parity-violating electron-proton scattering has been used to probe the structure of the proton in the non-perturbative regime of QCD. The parity-violating asymmetries are measured over a $Q^{2}$ range of 0.12 to $1.0(\mathrm{GeV} / \mathrm{c})^{2}$, with a beam energy of $3.028 \mathrm{GeV}$ and elastic proton scattering angles ranging from $52^{\circ}$ to $76^{\circ}$. The forward angle kinematics make the measured asymmetry relatively insensitive to axial-vector currents, and thus can be used to determine a linear combination of the charge and magnetization distribution of the strange quarks within the proton. Combining the present results with results from previous experiments provides significant constraints on the values of $G_{E}^{s}$ and $G_{M}^{s}$ at various $Q^{2}$.

The experimental results show strange quarks contributing to the charge and magnetization distribution of the proton at an $89 \%$ confidence level. All values of $G_{E}^{s}+\eta G_{M}^{s}$ measured in the present experiment can be found in Table 4.1. The strange quark contribution to the magnetization distribution of the proton at $Q^{2}=0.1(\mathrm{GeV} / \mathrm{c})^{2}$ is consistent with those from previous experiments and is measured to be $G_{E}^{s}\left(Q^{2}=0.1\right)=0.62 \pm 0.31$. The $Q^{2}$ dependence of $G_{E}^{s}+\eta G_{M}^{s}$ indicate a negative value for $G_{E}^{s}$ in the intermediate $Q^{2}$ region near $0.23(\mathrm{GeV} / \mathrm{c})^{2}$, and a positive linear combination at $Q^{2}$ above $0.5(\mathrm{GeV} / \mathrm{c})^{2}$. 


\section{Appendix A}

\section{Beam}

\section{A.1 Lasertable Studies}

During the summer-fall of 2003, tests of the IA and PZT were done on a mock laser table setup in the JLab Testlab in order to probe the stability of these two devices. A schematic of this setup can be seen in Fig. A.1. The Testlab laser table setup uses a Ti-Sapphire laser of the same model used in the injector. The IA and the PZT are set up such that the intensity and position modulations they induce can be monitored by a quadrant photo-diode (QPD). The QPD is a photodiode with breaks to divide it to form four independent quadrants (See Fig. A.1). When the laser spot hits the QPD, differing amounts of the spot can be distributed amongst the four quadrants. By measuring the relative intensities seen by each of the quadrants, the position of the beam with respect to the quadrants can be determined. The total intensity of the laser is simply the summed intensity of the four quadrants. The modulation frequency of the IA and PZT is $30 \mathrm{~Hz}$ as it is in the injector. Multiple measurements of the IA and PZT slopes over the course of weeks were made, and they were seen to be stable. Fig. A.2 shows these slopes. This result indicated that the source of the instability, particularly in the PZT-induced position differences, came from a change in beam tune over time. 

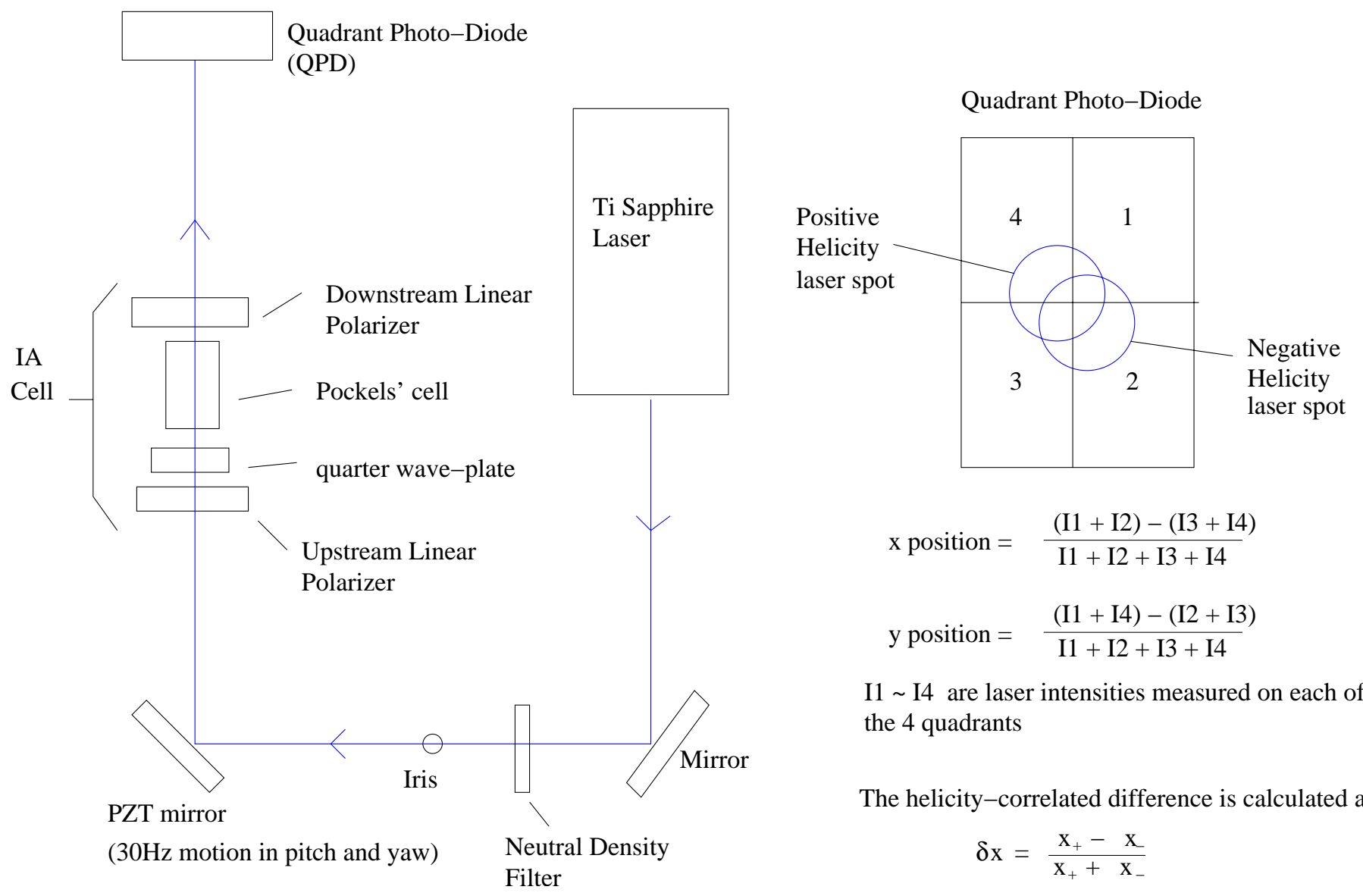

$$
\begin{aligned}
& \mathrm{x} \text { position }=\frac{(\mathrm{I} 1+\mathrm{I} 2)-(\mathrm{I} 3+\mathrm{I} 4)}{\mathrm{I} 1+\mathrm{I} 2+\mathrm{I} 3+\mathrm{I} 4} \\
& \mathrm{y} \text { position }=\frac{(\mathrm{I} 1+\mathrm{I} 4)-(\mathrm{I} 2+\mathrm{I} 3)}{\mathrm{I} 1+\mathrm{I} 2+\mathrm{I} 3+\mathrm{I} 4} \\
& \mathrm{I} 1 \sim \mathrm{I} 4 \text { are laser intensities measured on each of } \\
& \text { the } 4 \text { quadrants }
\end{aligned}
$$

The helicity-correlated difference is calculated as

$$
\delta x=\frac{x_{+}-x_{-}}{x_{+}+x_{-}}
$$

Figure A.1: The Testlab lasertable setup. The PZT and IA modulates the laser intensity and position at $30 \mathrm{~Hz}$. The laser then is monitored using a quadrant photo-diode.

\section{A.2 Beam Orthogonality}

Orthogonality is a measure of the transverse motion of the beam. In principle, if horizontal and vertical motions are to be induce on the beam at the cathode (as is the case with the PZT), the two motions should appear as perpendicular motions at the target in Hall C. However, if the two motions appear parallel to each other in the Hall, the PZT would lose its dynamic range along one of the transverse directions. This condition is called "orthogonality breaking". During the fallwinter 2003 test run, the orthogonality was measured both in the injector and in the Hall. Fig. A.3 shows the orthogonality of the beam as seen in the $100 \mathrm{keV}$ and $5 \mathrm{MeV}$ sections of the injector. Looking at the first BPM after the cathode, orthogonality is maintained. However, toward the end of the $100 \mathrm{keV}$ region to the transition between the $100 \mathrm{keV}$ and $5 \mathrm{MeV}$ region, there is a relative 

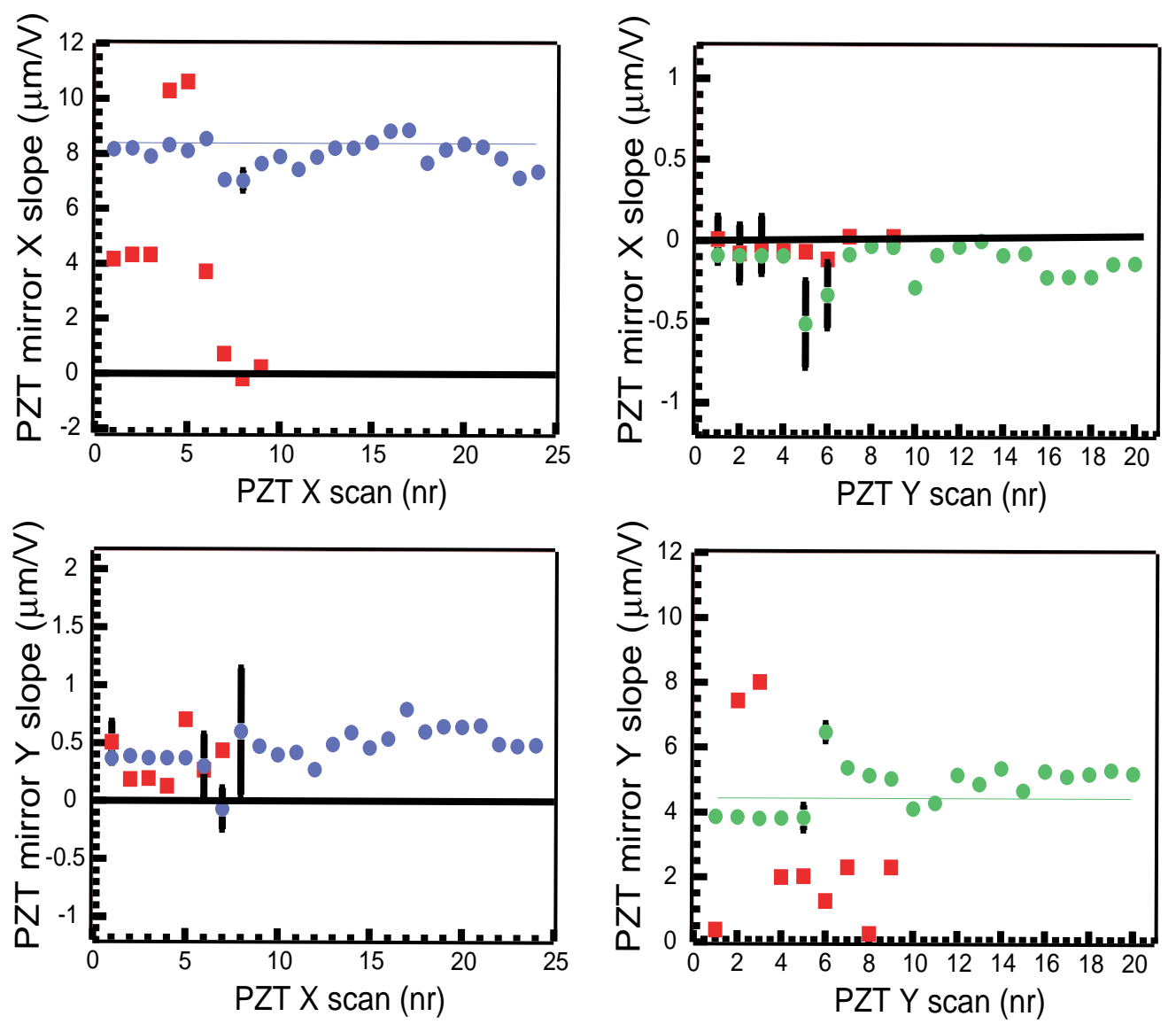

Figure A.2: PZT slopes measured over time on the Testlab laser table.. Red points indicate measurements taken when the setup is significantly altered, such as changes in distance from the PZT to the QPD, as well as re-alignment of the IA cell.

rotation between the two vectors, causing orthogonality breakdown. Asymmetric magnetic fields along the beamline (caused by poorly centered elements, for example) can cause such a problem. However, as in the case of the lack of adiabatic damping, the $100 \mathrm{keV}$ to $5 \mathrm{MeV}$ region of the machine is not understood well enough to explain this rotation. At the $G^{0}$ target in Hall $\mathrm{C}$, the orthogonality is somewhat better than what is seen in the $5 \mathrm{MeV}$ region of the injector. Fig. A.4 shows the orthogonality of the PZT signals in the Hall. 

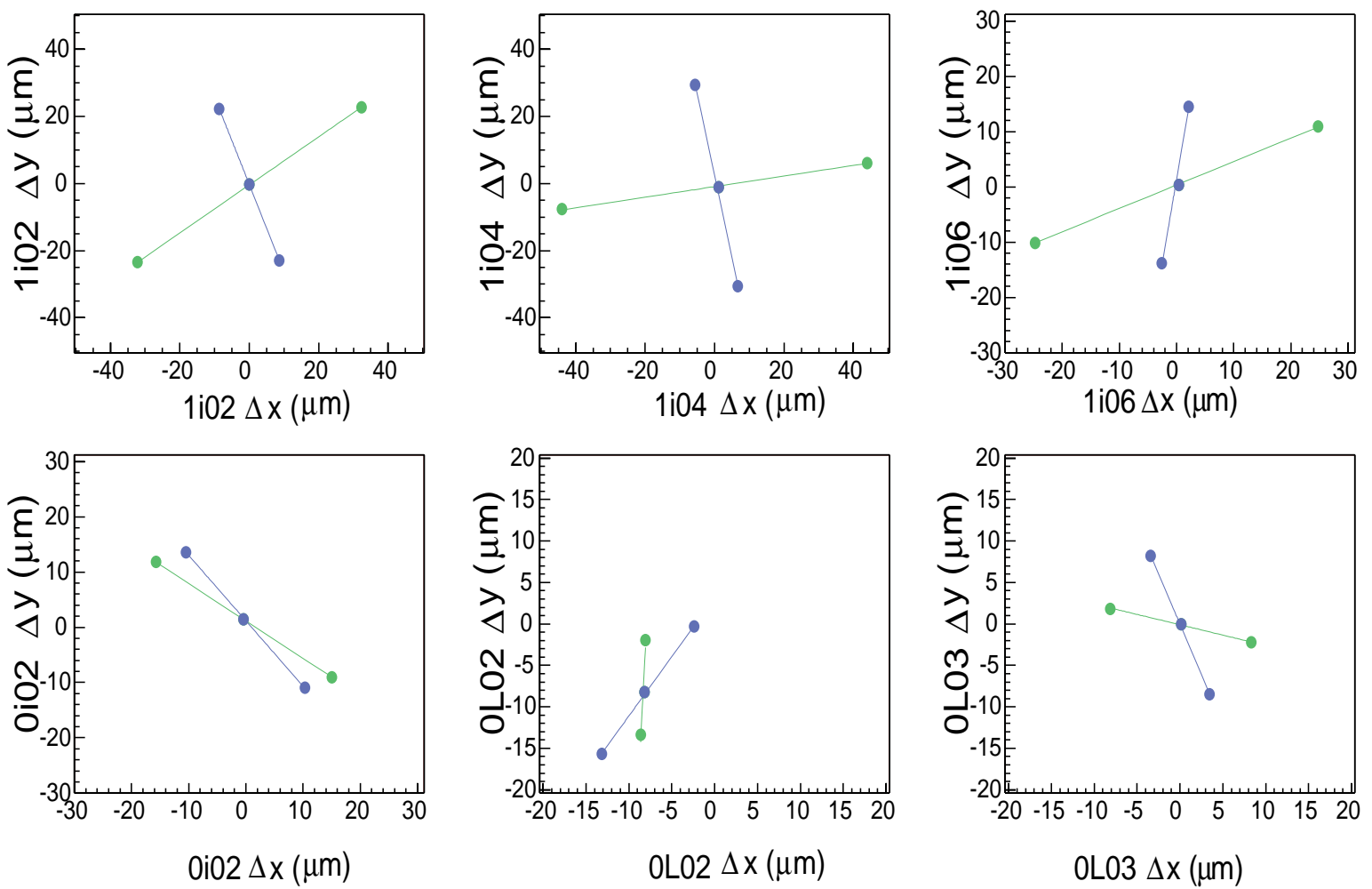

Figure A.3: Beam orthogonality as seen in the injector. The BPMs are left to right, top to bottom in the order they appear in the injector. Green shows $\mathrm{x}$ and y position differences induced with PZTx motion, and blue is that of PZTy motion. The first four BPMs (1i02, 1i04, 1i06, and 0i02) are located in the $100 \mathrm{keV}$ section, and the last two (0L02, 0L03) are located in the $5 \mathrm{MeV}$ region of the injector.

\section{A.3 Quadrupole Adjustments}

Quadrupoles located in Hall $\mathrm{C}$ are used to focus the beam in order to bring the existing position differences down to the point where they can be fed back with the PZT. Since quadrupoles focus along one transverse plane and defocus along the transverse plane perpendicular to the first, quadrupole adjustments are typically made in pairs in order to induce a net focusing along both planes.

An underlying assumption in the use of quadrupoles to reduce position differences is that they do not in themselves induce additional helicity-correlated steering as the electron passes through it. Strictly speaking, this is not true due to the opposing spin (and hence, magnetic moment) of the two helicity states. Since the wien filter forces the electron spin to be (anti-)parallel to the direction of motion only at the target, any transverse spin components that the electron may possess when it 


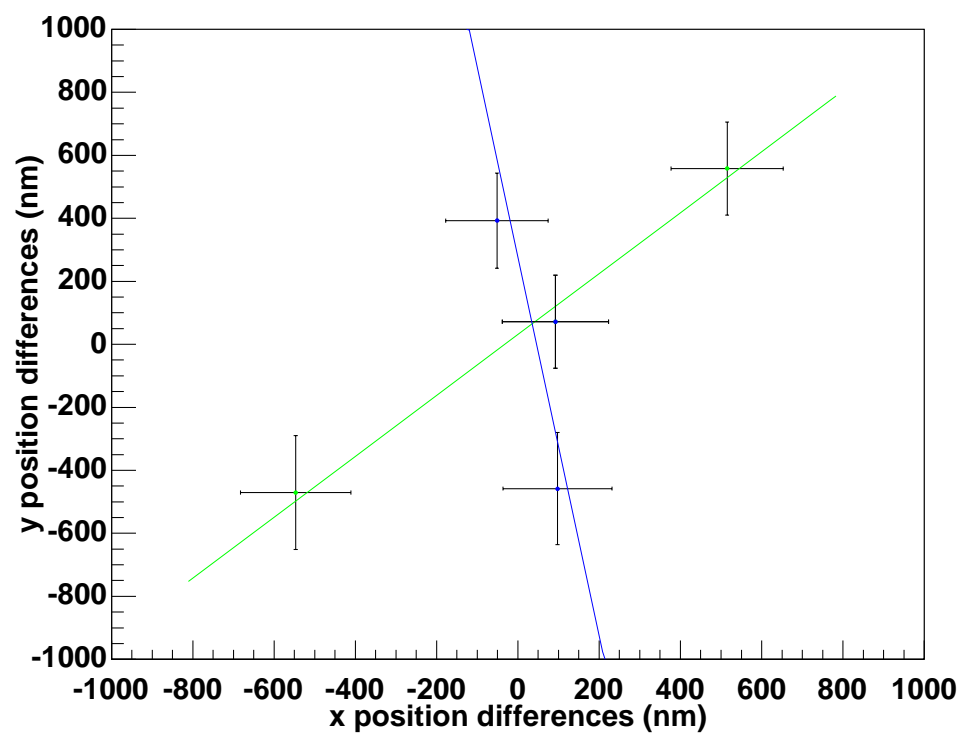

Figure A.4: Orthogonality of the PZT signals in Hall C. Green is PZTx and blue is PZTy. The angle made by the two signals here is 80.9 degrees.

passes through the quadrupole could couple to the magnetic field gradient. The force experienced by the electron due to its spin in such a case would be

$$
F= \pm \mu_{e} \frac{d B}{d x}
$$

where $\frac{d B}{d x}$ is the field gradient, and $\pm \mu_{e}$ is the magnetic moment of the electron along the field gradient. However, such an effect is suppressed by the fact that the quadrupoles used in the hall are near the target, where the beam polarization is longitudinal.

The effect of the quadrupole magnets on the position differences can be best seen by observing the RMS noise in these position differences. Fig. A.5 shows a case where two quadrupole magnets are adjusted in order to reduce the noise in the Y position differences. The quadrupoles are adjusted to minimize the RMS noise in the position differences. The results are that the statistical uncertainty on the position differences are smaller during each iteration of PZT feedback (resulting in a more effective feedback), and that the average position differences themselves decrease (requiring less work from the PZT). 

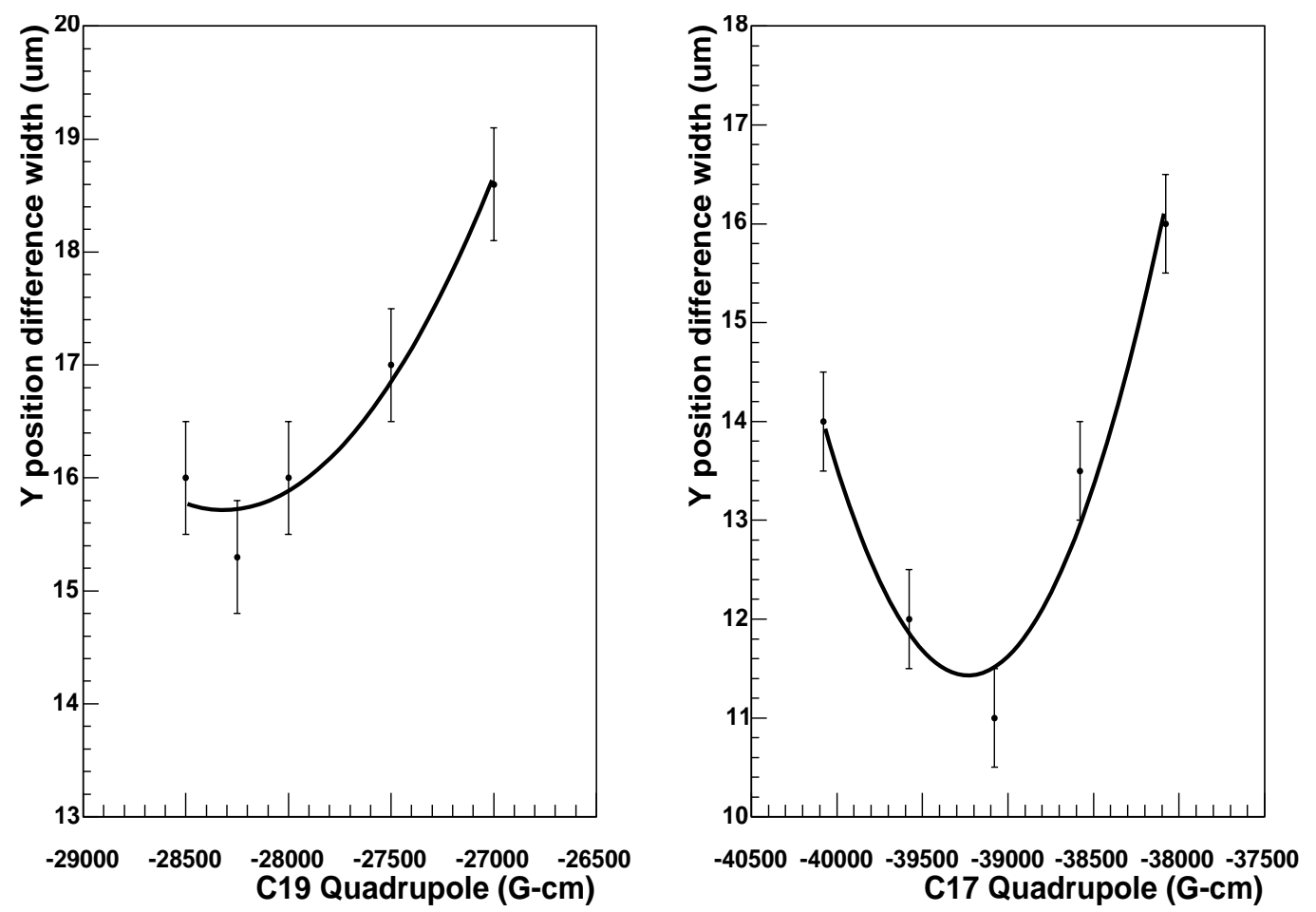

Figure A.5: Variation of the width of the y position differences as two quadrupole magnets (3C17 and $3 \mathrm{C} 19)$ are adjusted. The quadrupole fields of each are set to minimize the width of the position differences.

\section{A.4 Beam Correlations}

During calibrations of the IA and the RHWP, a correlation between $A_{q}$ and $\delta_{x(y)}$ that is independent of either devices is seen. Both the IA and the RHWP induce charge asymmetry in the beam, albeit through completely different mechanisms. The IA directly modulates the intensity of the laser, while the RHWP changes the orientation of the residual linear polarization of the laser and relies on the analyzing power of the cathode to change the resulting beam intensity. Since these two mechanisms are different, the position differences induced by these two devices should not be correlated. However, the correlation between the charge asymmetry and position difference produced by these two devices were measured to be the same as seen in Fig. A.6. Thus, this correlation is thought to originate after the cathode, and is expected to be through scraping.

This correlation directly affects the first row of the $3 \times 3$ feedback matrix which characterizes the IA. Since the feedback matrix assumes there are no direct correlations between charge asymmetry and position differences, this additional correlation will interfere with the operation of the feedback 

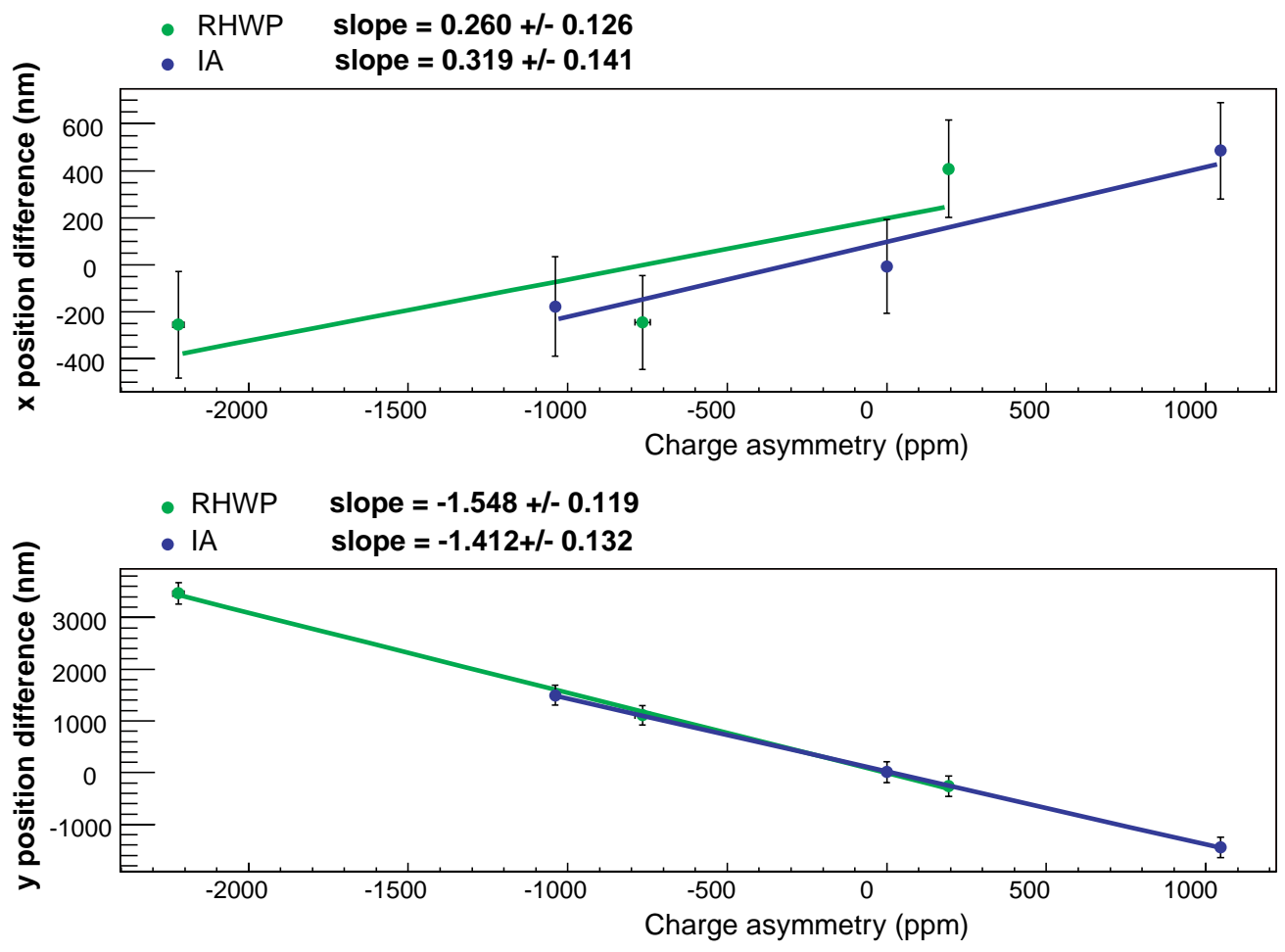

Figure A.6: Correlation between $\mathrm{x}$ and $\mathrm{y}$ position differences and charge asymmetry for an IA and an RHWP scan. Blue is from the IA calibration, while green is from that of the RHWP.

system. Therefore, the parts of the matrix which characterize the IA induced position differences must be corrected for this additional correlation that the beam acquires in the machine. The original slopes are measured through an IA scan as

$$
\frac{d I A}{d \delta x}_{\text {measured }}=\frac{V_{1}-V_{2}}{\delta x_{1}-\delta x_{2}}
$$

where $\delta x_{1(2)}$ the position difference induced when a voltage $V_{1(2)}$ is applied to the IA. In order to correct for the additional position differences induced by charge asymmetry, the correlation as 
measured by the RHWP is used to calculate the new IA slopes.

$$
\frac{d I A}{d \delta x} \text { corrected }^{=} \frac{V_{1}-V_{2}}{\left(\delta x_{1}-\frac{d \delta x}{d A_{q}} A_{q 1}\right)-\left(\delta x_{2}-\frac{d \delta x}{d A_{q}} A_{q 2}\right)}
$$

where $\frac{d \delta x}{d A_{q}}$ is the charge asymmetry-position difference correlation measured by the RHWP.

Fig. A.7 and A.8 shows the performance of the feedback mechanism immediately before and after this correction was made to the feedback matrix.
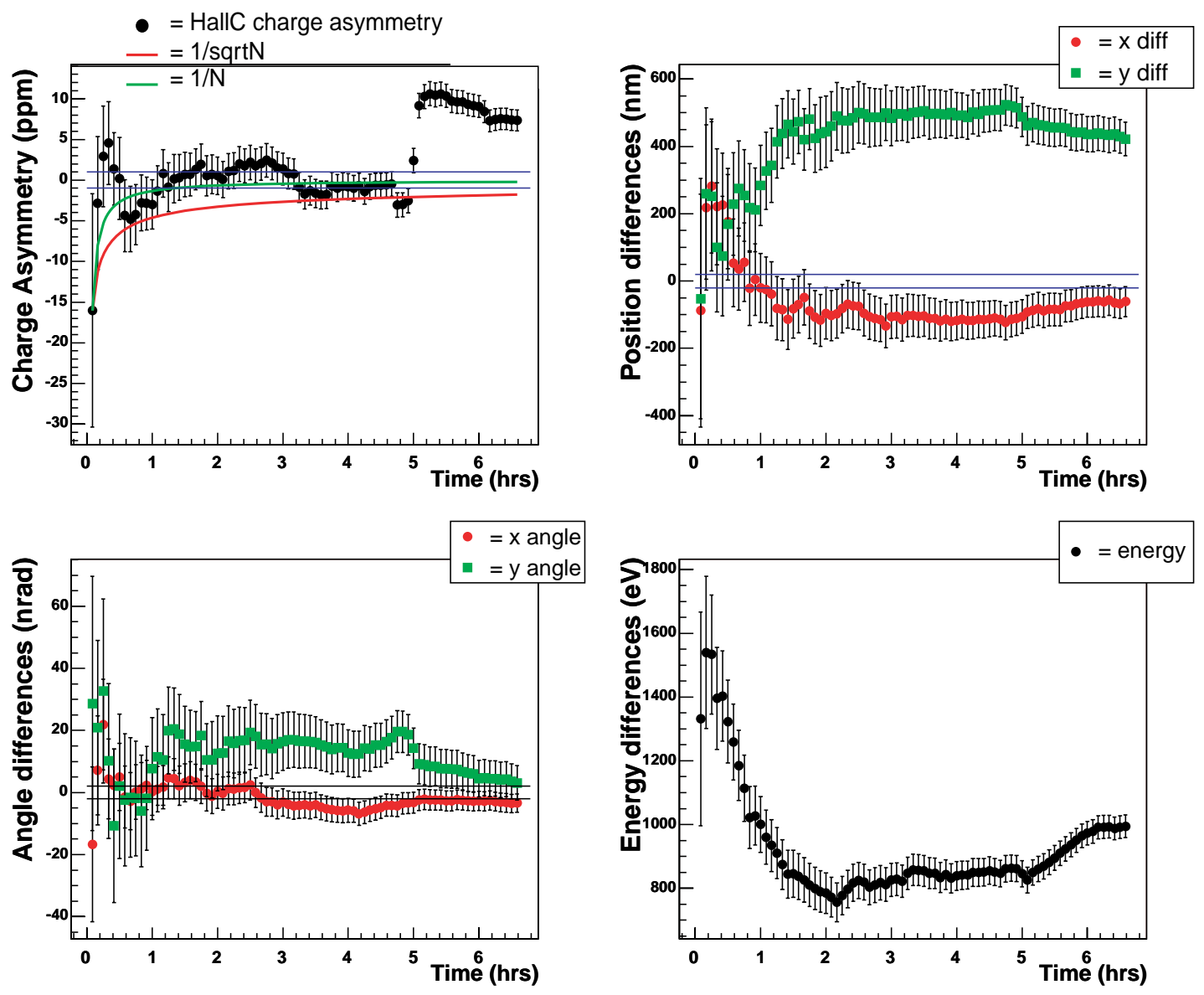

Figure A.7: Feedback performance before the correction of the feedback matrix. The position difference is clearly not converging. The feedback software tried to set the IA and PZT beyond their voltage range 5 hours after the algorithm was started. This prompted a manual restart of the feedback code as indicated by the sudden spike in charge asymmetry. 

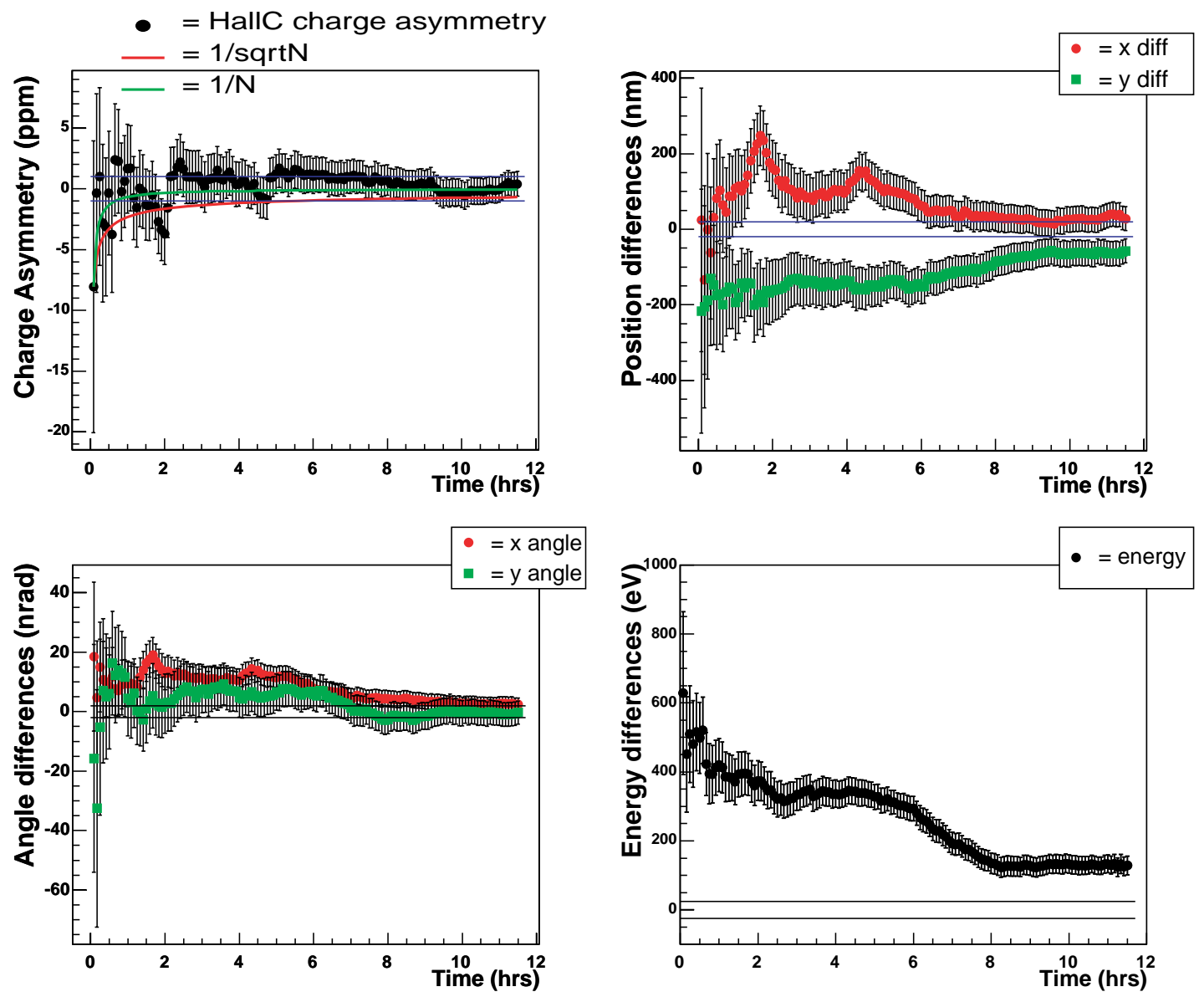

Figure A.8: Feedback performance after the correction of the feedback matrix. The feedback system is seen to be clearly reducing the position differences. 


\section{Appendix B}

\section{Electronics}

\section{B.1 Musson box systematics}

As in the case of the HC beam parameters, the signals coming from the Musson box must also be independent of the helicity signal. Since the $Y_{0}$ and CLK signals are both derived from the 1499 $\mathrm{MHz}$ RF cavity signal, there is a possibility that the Musson box signals can couple to one of the HC beam parameters. This can cause helicity-correlated time offsets to the Musson box signals. Sensitivity to beam parameters is measured by using a TDC to compare the $Y_{0}$ time with the time of a $31 \mathrm{MHz}$ signal produced from the master oscillator, which provides the reference frequencies for the RF cavities. The bin width of the TDC is approximately $35 \mathrm{ps}$ and 31 measurements are made and averaged together for each MPS. This $Y_{0}$ TDC time is then plotted against the beam current and positions to see if there are any correlations. Fig. B.1 and B.2 shows that there is no visible dependence. The charge correlation is about $1.2 \mathrm{ps} / \mu \mathrm{A}$, which corresponds to a helicity-correlated time offset of $1.2 \mathrm{ps} / \mu \mathrm{A} \times 0.14 \mathrm{ppm} \times 40 \mu \mathrm{A}=0.007 \mathrm{fs}$. The corresponding position correlation is about $20 \mathrm{ps} / \mathrm{mm}$, which translates to a helicity-correlated time offset of $20 \mathrm{ps} / \mathrm{mm} \times 4 \mathrm{~nm}=0.08$ fs. These time offsets are small enough to be negligible systematics.

Further tests have been performed by directly changing the path-length of the electron orbit (using a dogleg), and determining the resulting change in the beam arrival time is reflected by a change in time of the $Y_{0}$ and CLK signals. The result of this test showed that the Musson box responds as expected. 


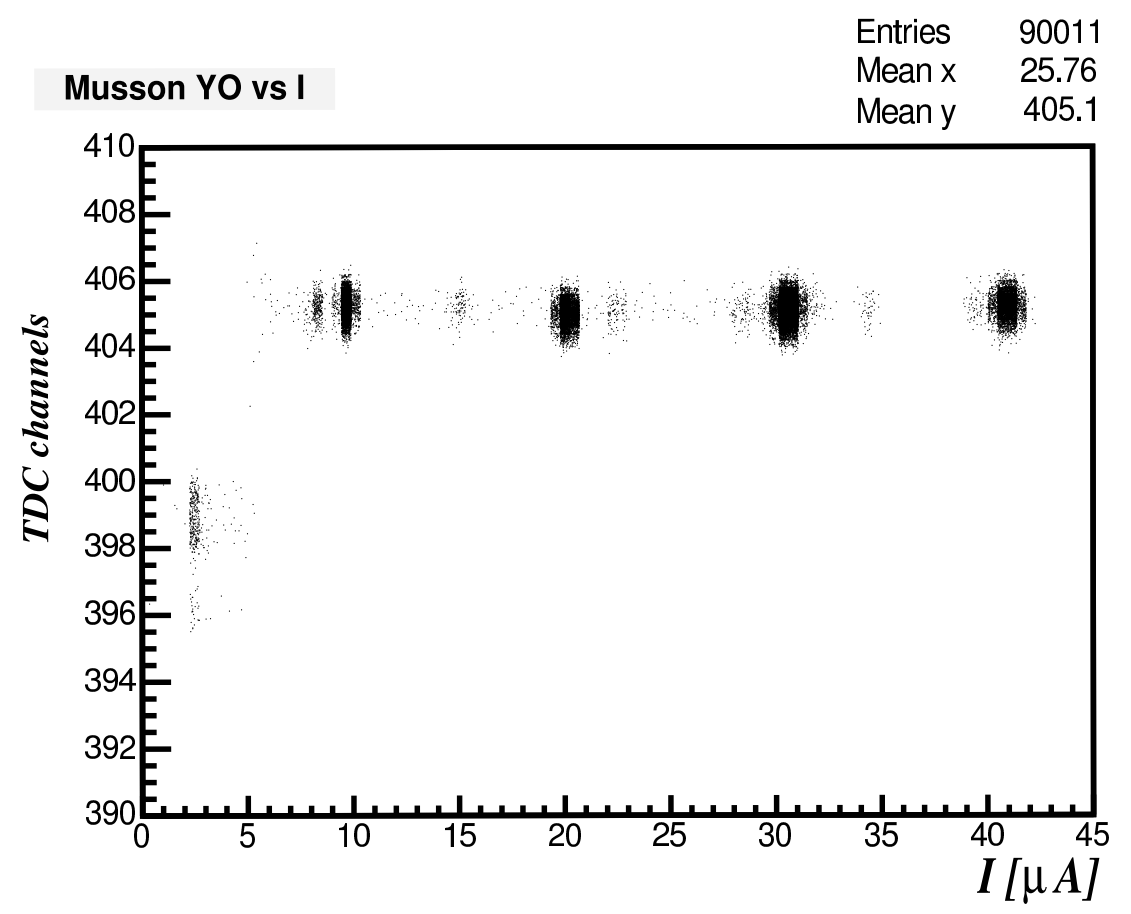

Figure B.1: $Y_{0}$ vs Beam current. No visible correlation is seen.
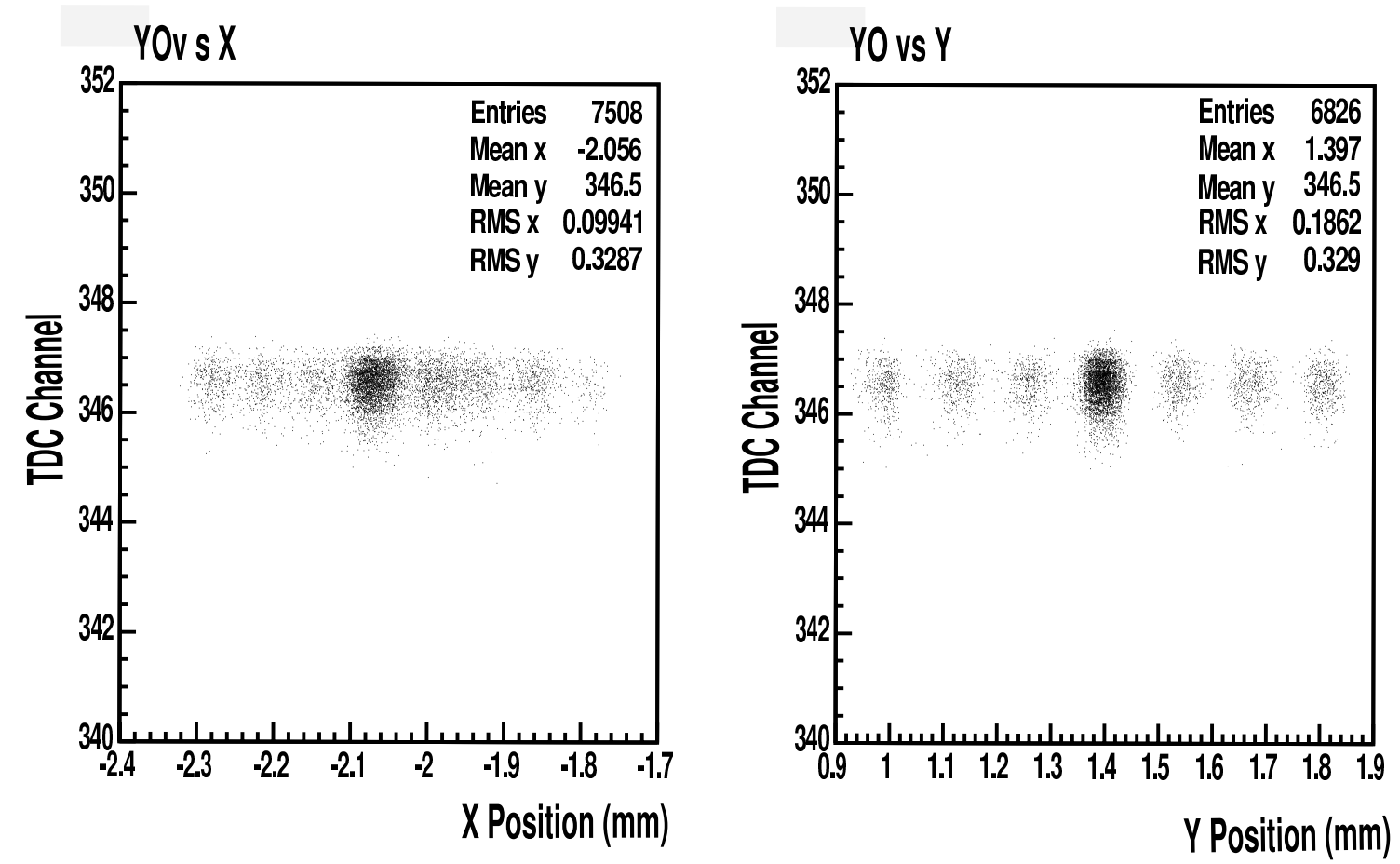

Figure B.2: $Y_{0}$ vs Beam position. No visible correlation is seen. 


\section{B.2 DNL}

The differential non-linearity measured during the first engineering run was seen to fluctuate over time. The impact of this effect is two-fold. On one hand, the PID cuts as well as the dilution of the asymmetry by the background contribution were time dependent and would have required repetitive calibrations. In addition, the precision of the binning in $Q^{2}$ of the data of detector 15 would have been significantly reduced. Here, the use of a more stable $Y_{0}$ signal significantly reduced this effect for the production run.

In order to determine how much the bin widths shift relative to each other, a tof spectrum is constructed for each detector with a white noise source. In principle, the spectrum from such a source should be flat. However, any non-zero DNL would result in bin width-dependent fluctuations. Fig. B.3 shows a tof spectrum seen from such a source. The DNL is thus measured intermittently

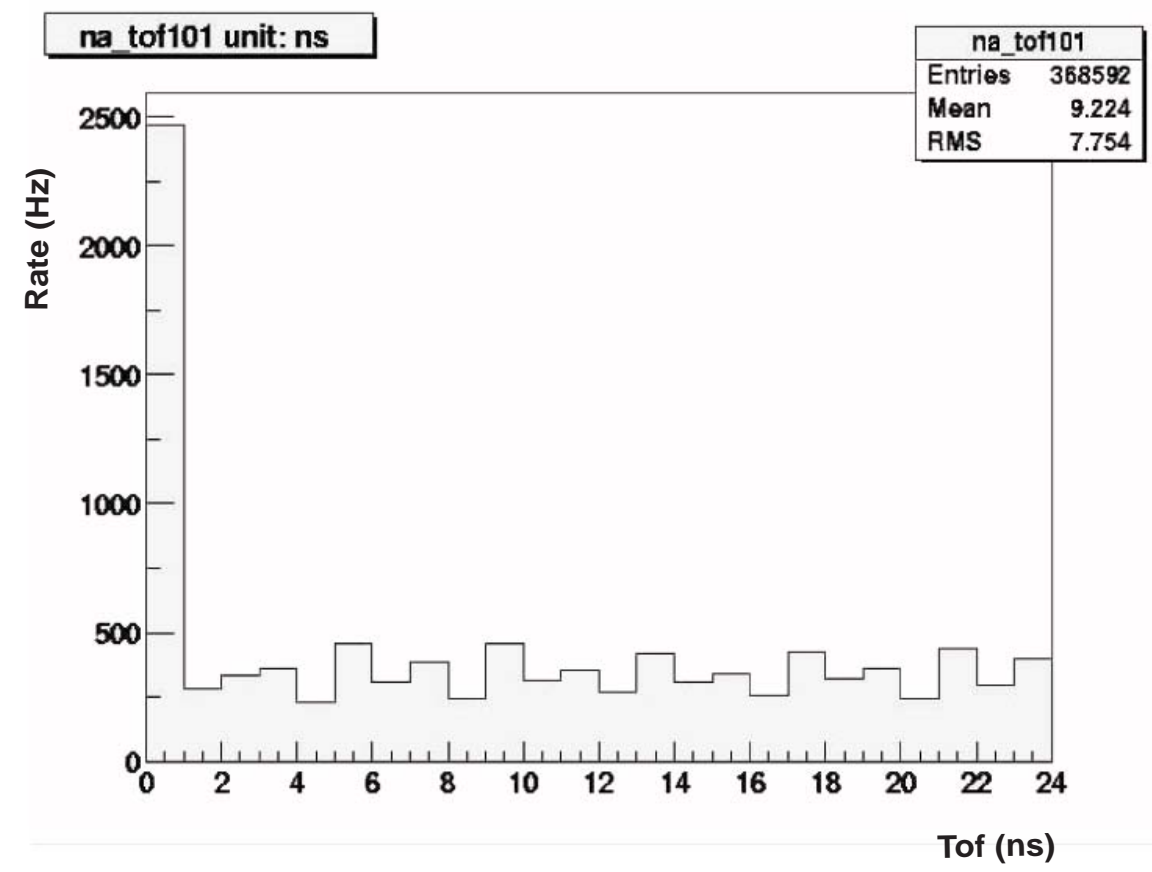

Figure B.3: White noise spectra for one of the NA detectors. Fluctuations result from uneven bin widths across the spectra. The large rate at $0 \mathrm{~ns}$ is artificial. Since the NA detectors only look at 24 of the $32 \mathrm{~ns}$ time spectrum, all rates from the remaining $8 \mathrm{~ns}$ are seen in the first bin.

over the entire production run, and the bin widths were corrected according to the fluctuations seen in each spectrum. In order to assess the stability of DNL, the asymmetry of the yield seen in 
even and odd bins were determined and tracked over time. Fig. B.4 shows the improvement seen in the DNL (due to the improved $Y_{0}$ ) between the first and second engineering runs.

DNL Octant 7 Detector 9

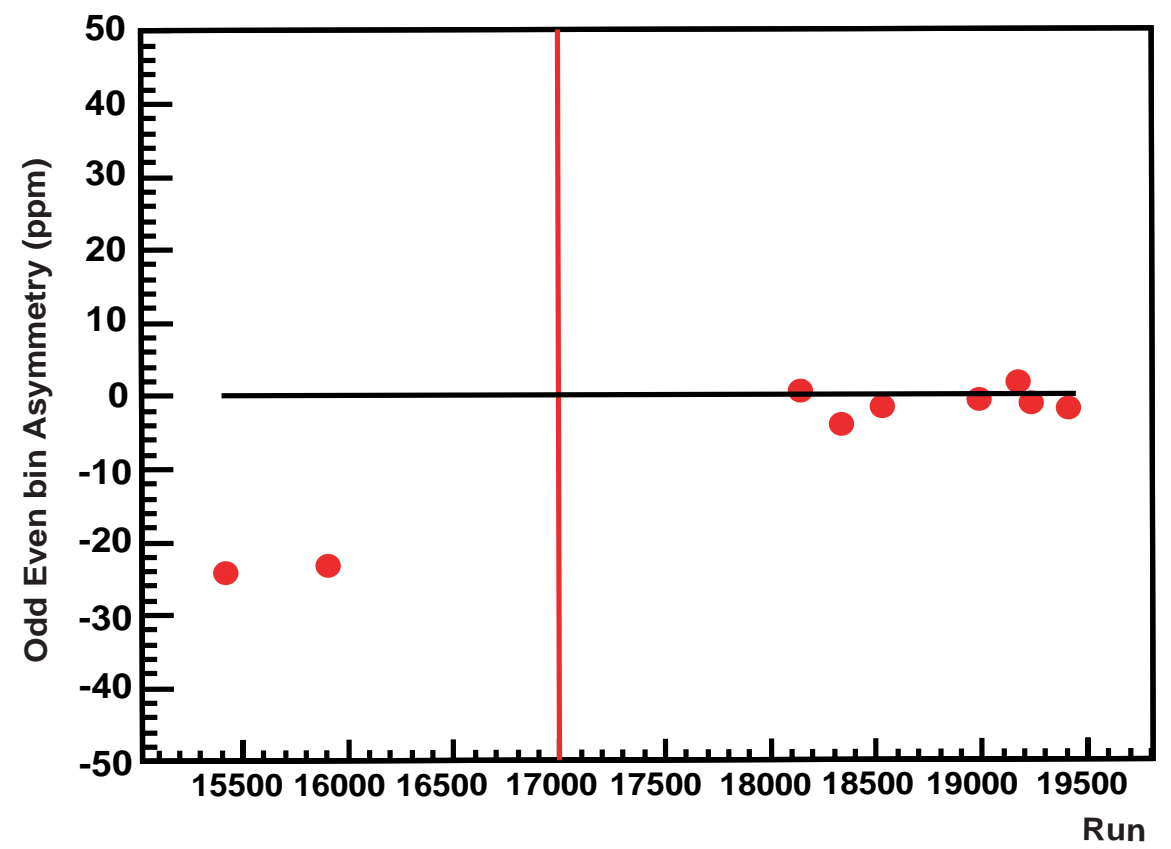

Figure B.4: Improvement of DNL from the first to the second engineering run. The points show the asymmetry in the yield between even and odd bins. The red line separates runs taken during the two runs. Marked improved is seen between the first and second engineering runs.

\section{B.3 Electronics Efficiency}

At each reading of the TEE, the modules produce a bit signaling their performance during the last integration period. The malfunctions that triggers the bit can be due to bad external timing (from a bad $Y_{0}$ signal), marginal pulse structure for the NA electronics, and unacceptably high instantaneous rates for the FR electronics.

An unstable $Y_{0}$ or CLK signal would cause problems for the NA LTDs, since the shift registers for the LTDs rely on the CLK signal, and the tof start time is dependent on the $Y_{0}$. Any significant deviation in the rates seen in the LTD channels trigger the aforementioned bit, indicating a problem. This problem was mitigated between the first and second engineering runs by providing a better $Y_{0}$ and CLK signal via the Musson box, and implementing a more stable level translator for the 
NA electronics.

One of the issues seen in the FR electronics is that the FR electronics occasionally sees high instantaneous rates in one or more of their time bins. Fig. B.5 shows an example of this "spike" effect. The error bits in the FR electronics detects the presence of these spikes in the datastream.

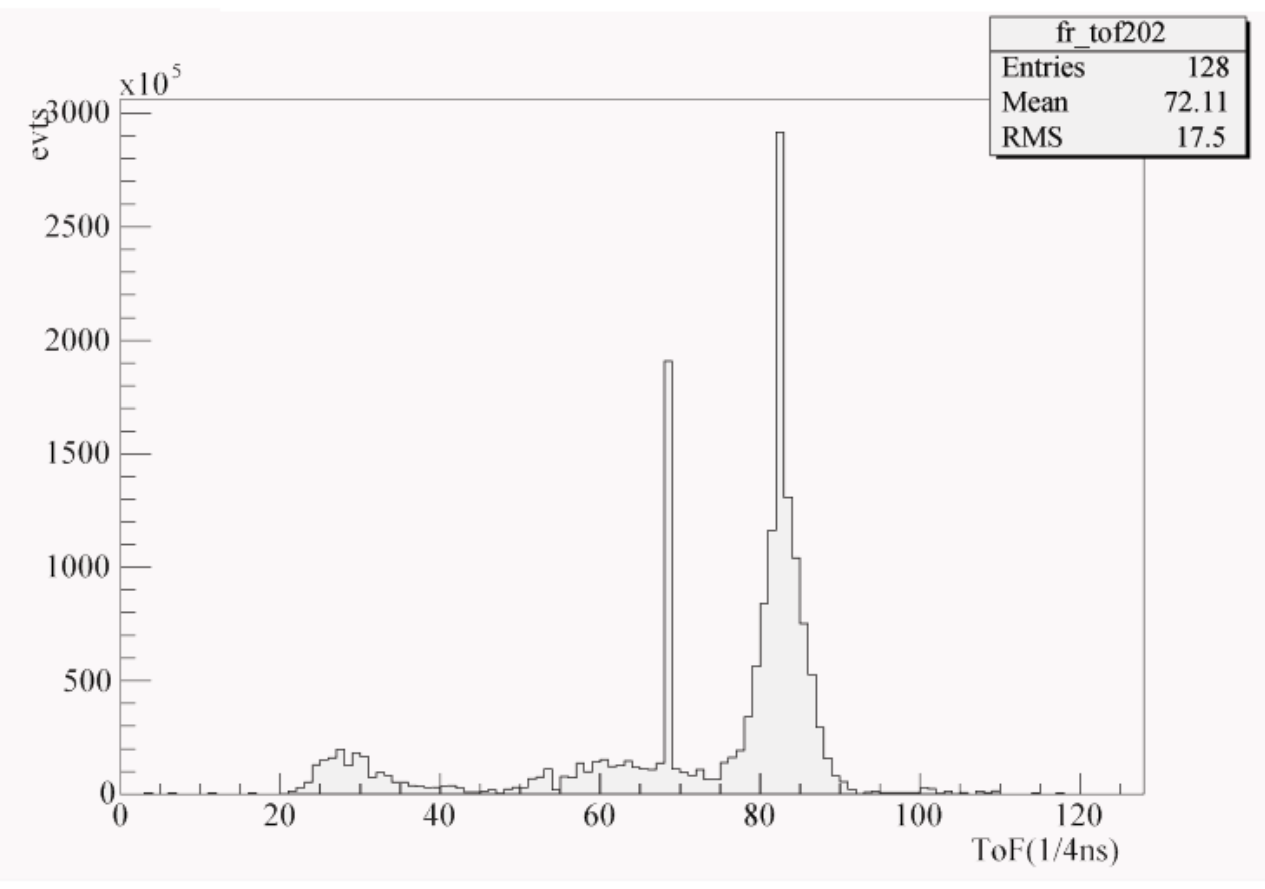

Figure B.5: Example of a spike in the FR electronics. Two prominent spikes can be seen in this plot. One at time bin 63 , and another at time bin 82 (on top of the elastic peak).

The detector efficiency is then defined to be the ratio between the number of readings without errors to the total number of readings. Fig. B.6 shows this efficiency for a set of FR and NA detectors over several runs. Overall, the detector efficiencies are seen to be stable. Data where error bits were seen have been cut at the analysis level to ensure they do not affect the results. 

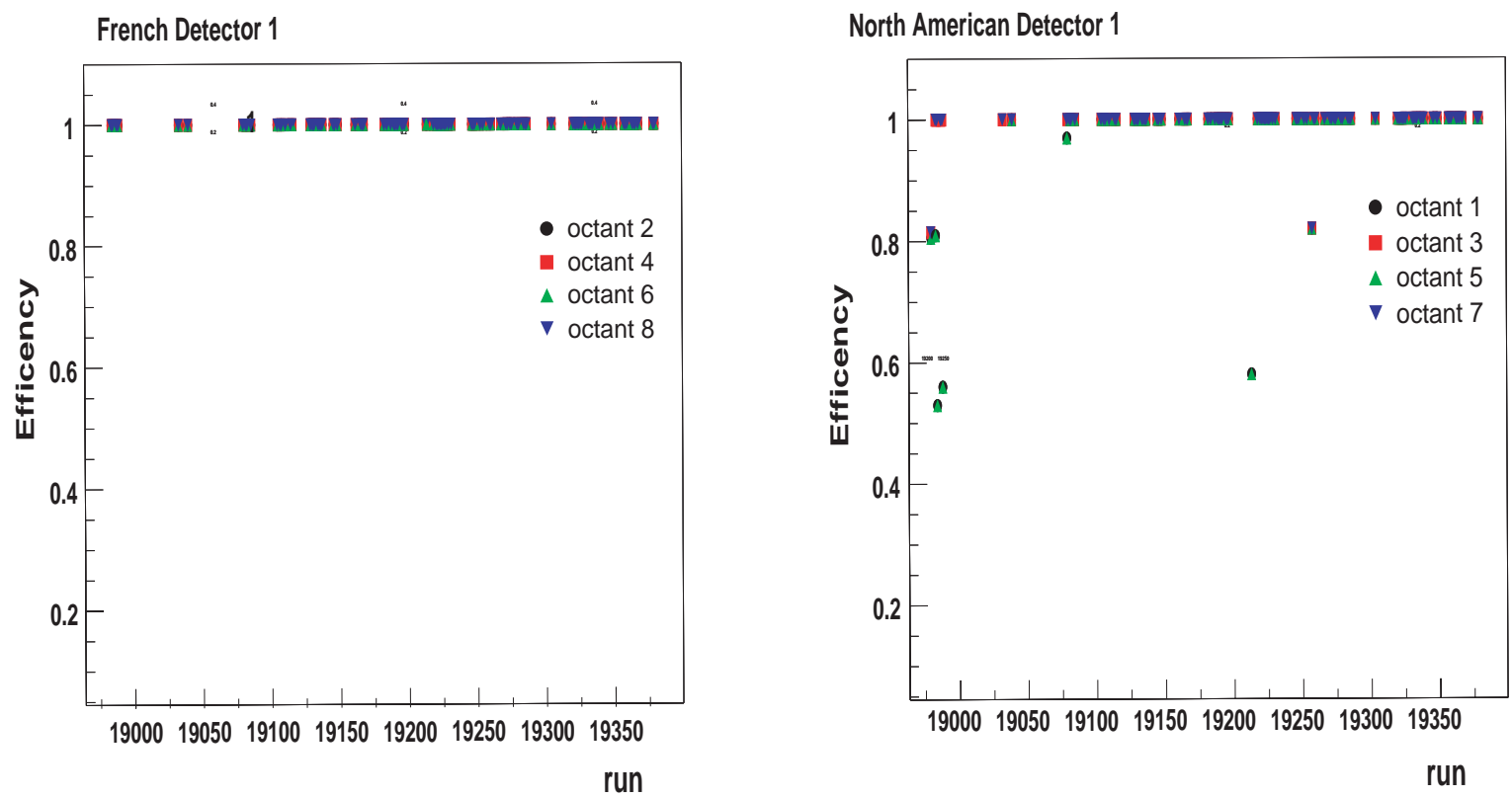

Figure B.6: FR and NA detector efficiency. Deviations from one show runs where the error bit is triggered. 


\section{Appendix $\mathrm{C}$}

\section{Analysis}

\section{C.1 Tof Spectra}

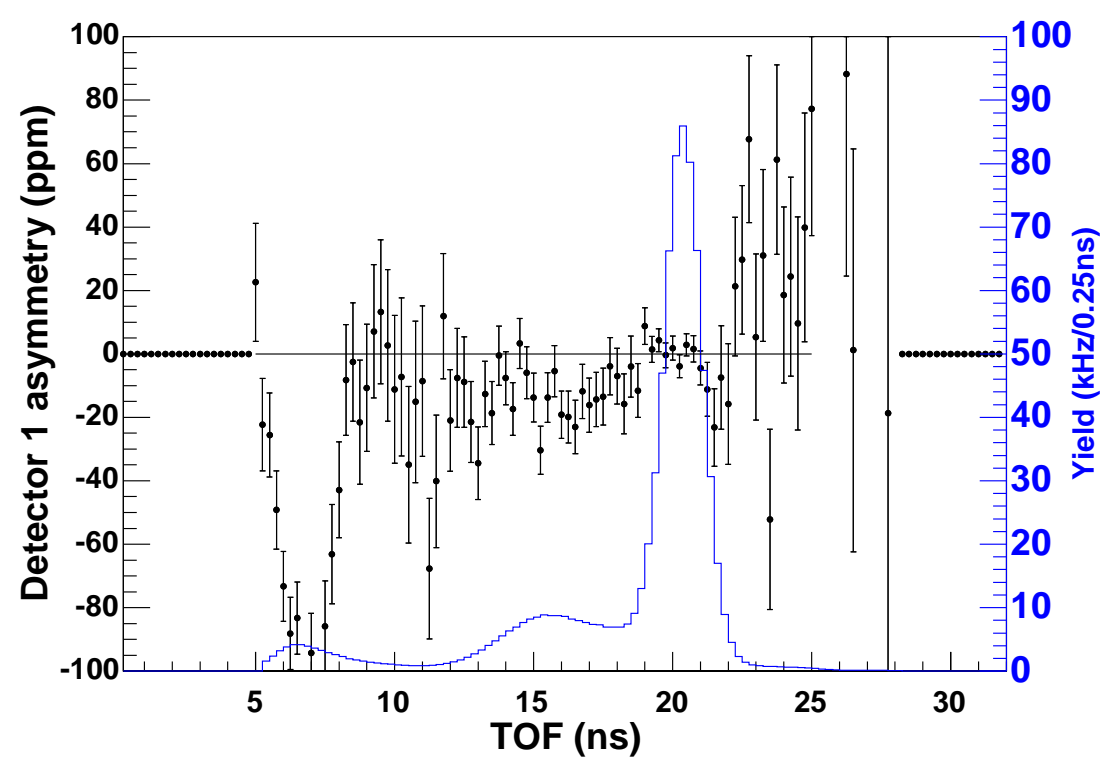

Figure C.1: Octant 2 Detector 1. Asymmetries (black), and yields (blue). 


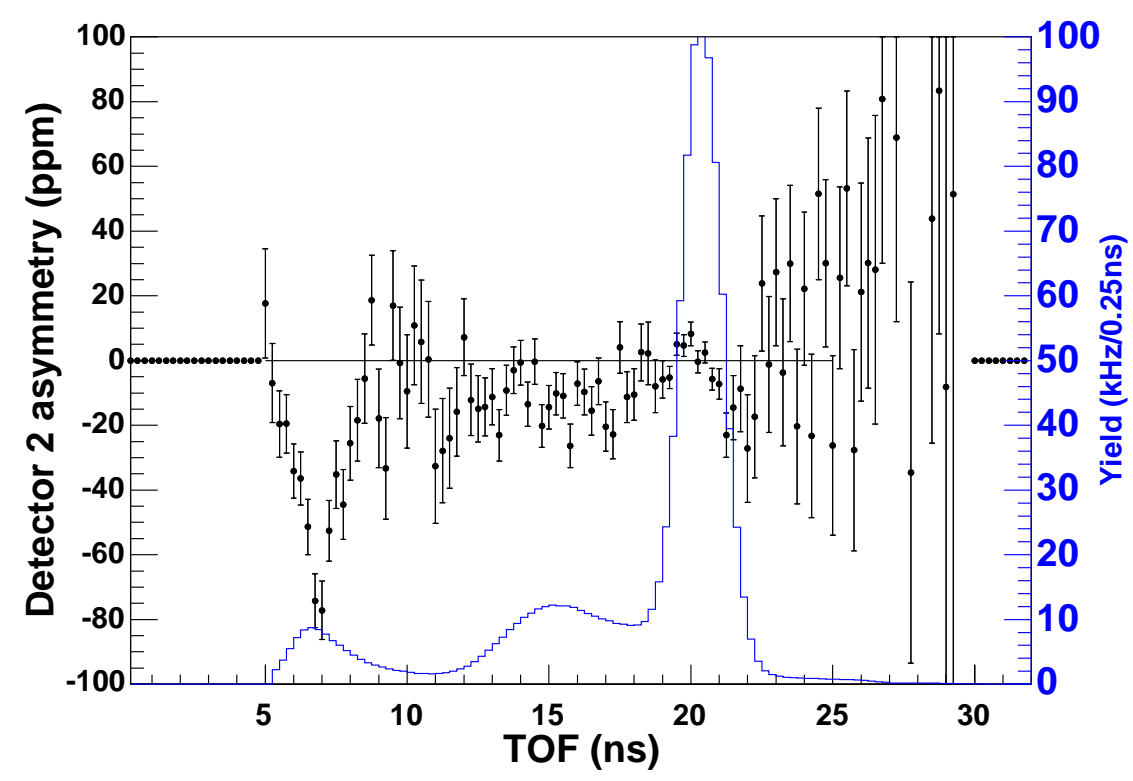

Figure C.2: Octant 2 Detector 2. Asymmetries (black), and yields (blue).

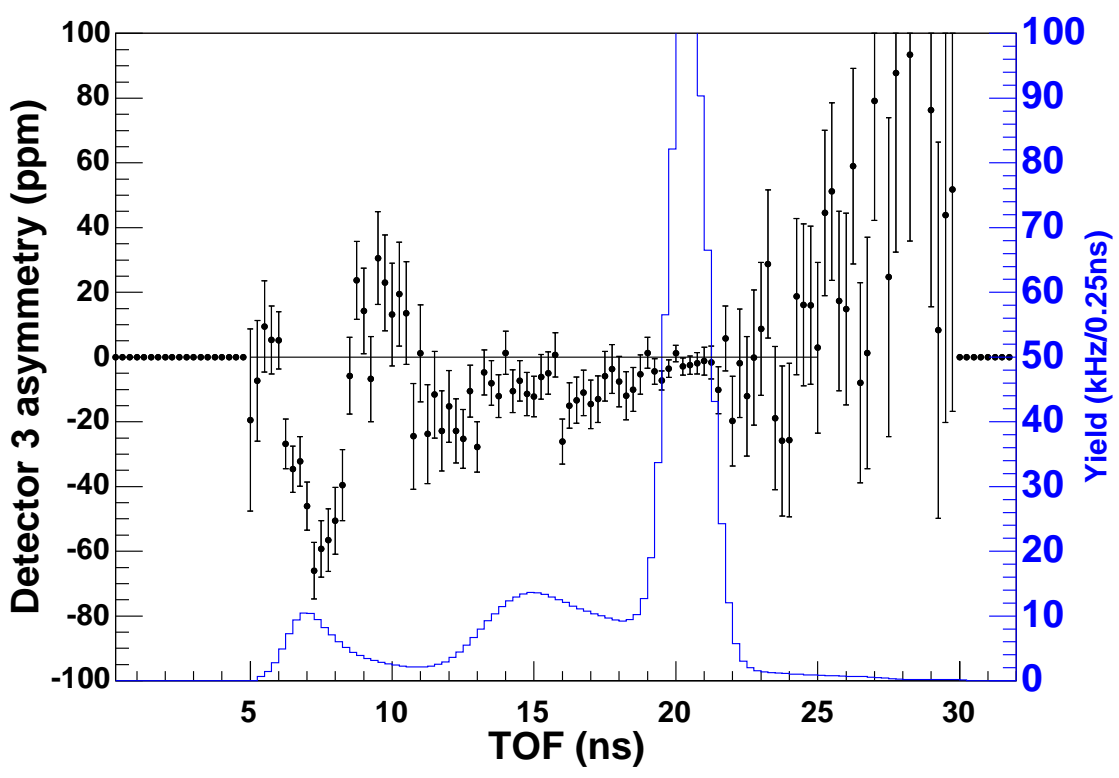

Figure C.3: Octant 2 Detector 3. Asymmetries (black), and yields (blue). 


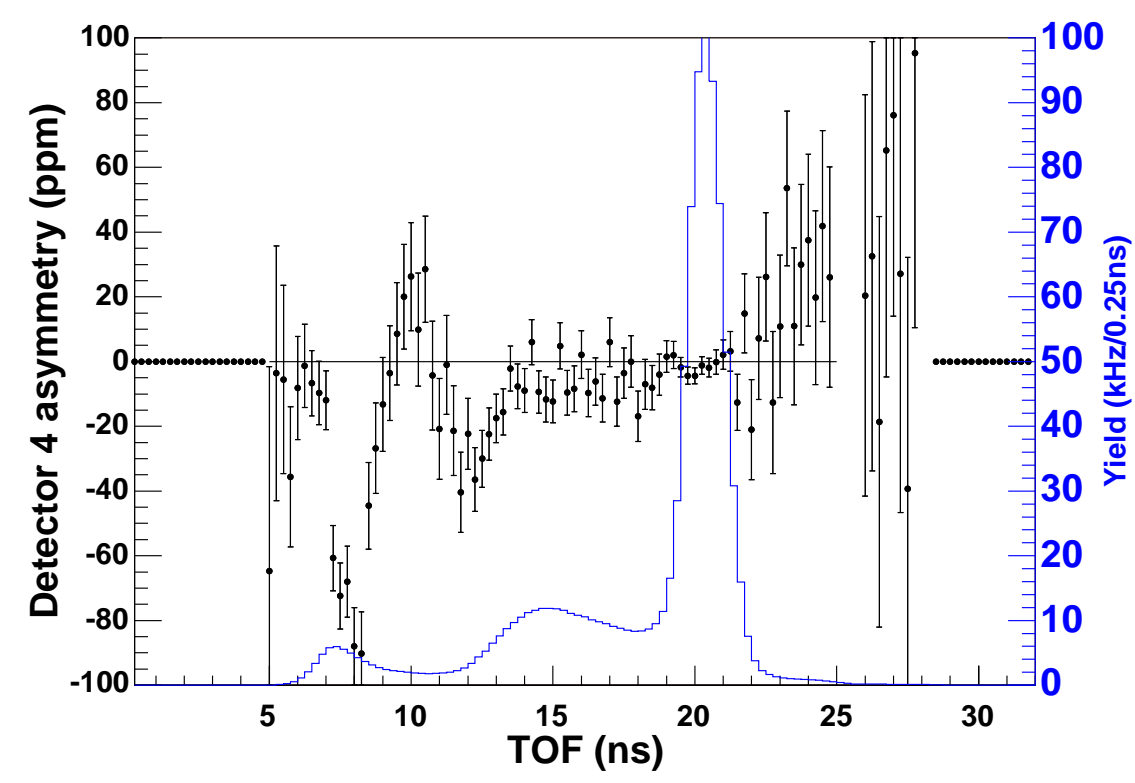

Figure C.4: Octant 2 Detector 4. Asymmetries (black), and yields (blue).

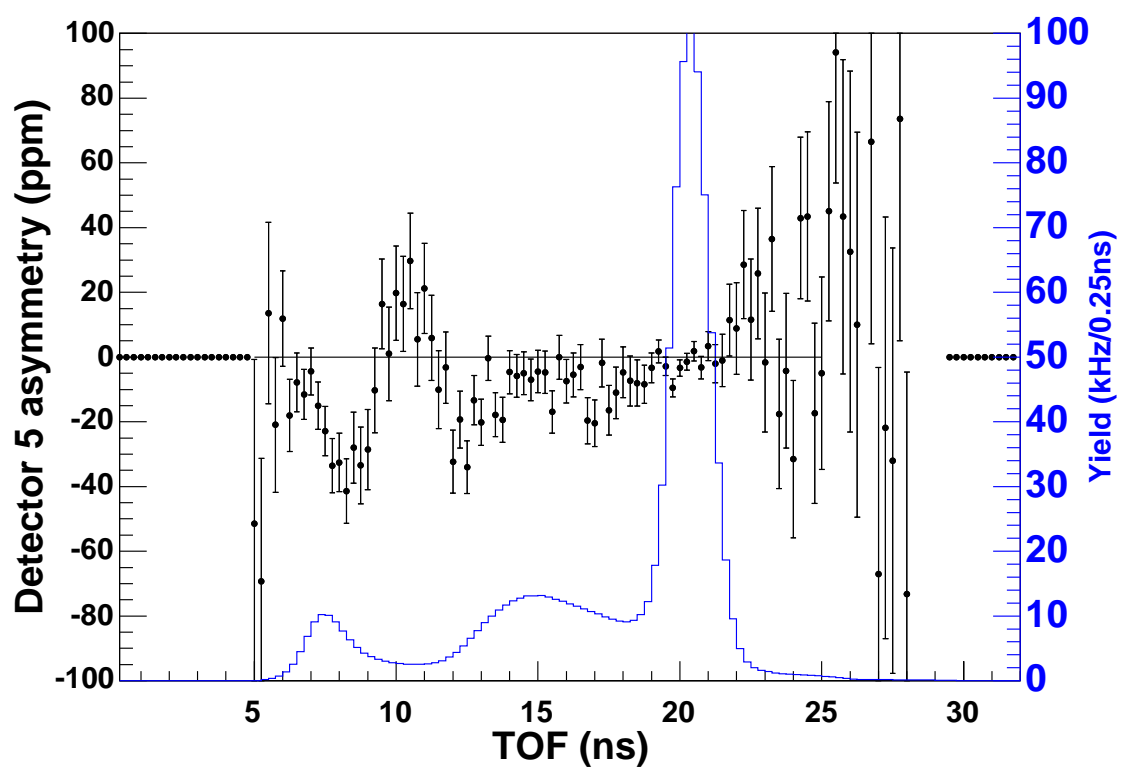

Figure C.5: Octant 2 Detector 5. Asymmetries (black), and yields (blue). 


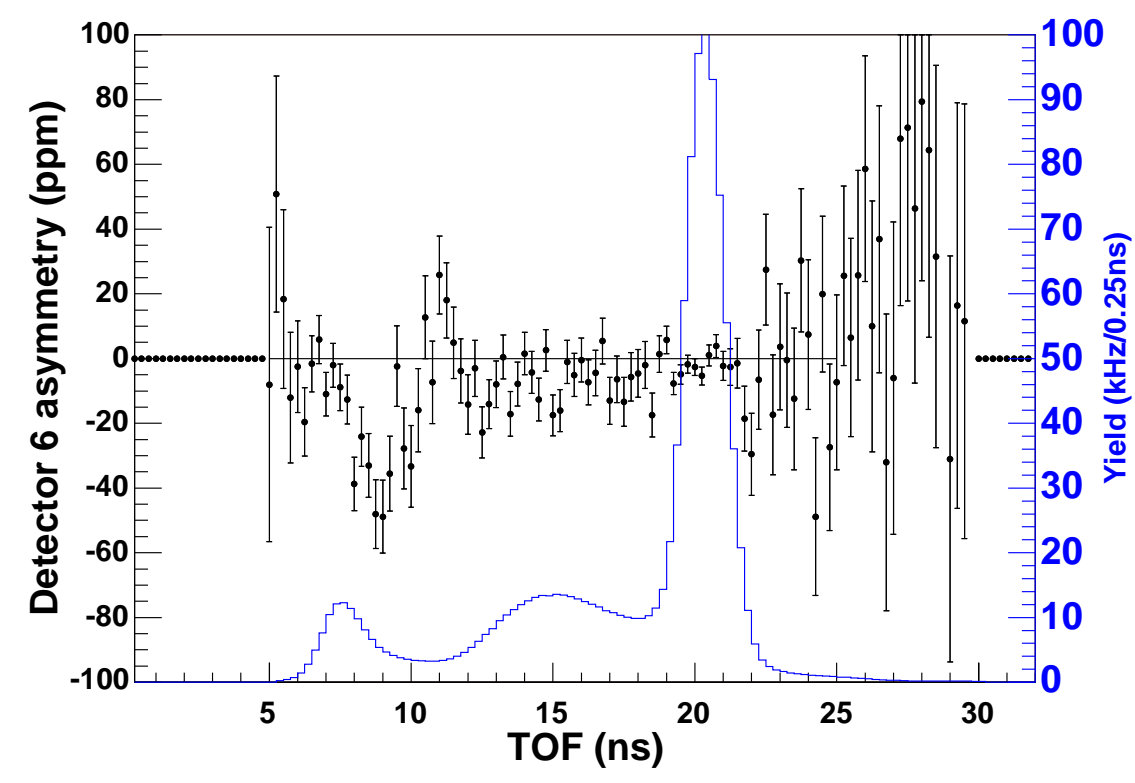

Figure C.6: Octant 2 Detector 6. Asymmetries (black), and yields (blue).

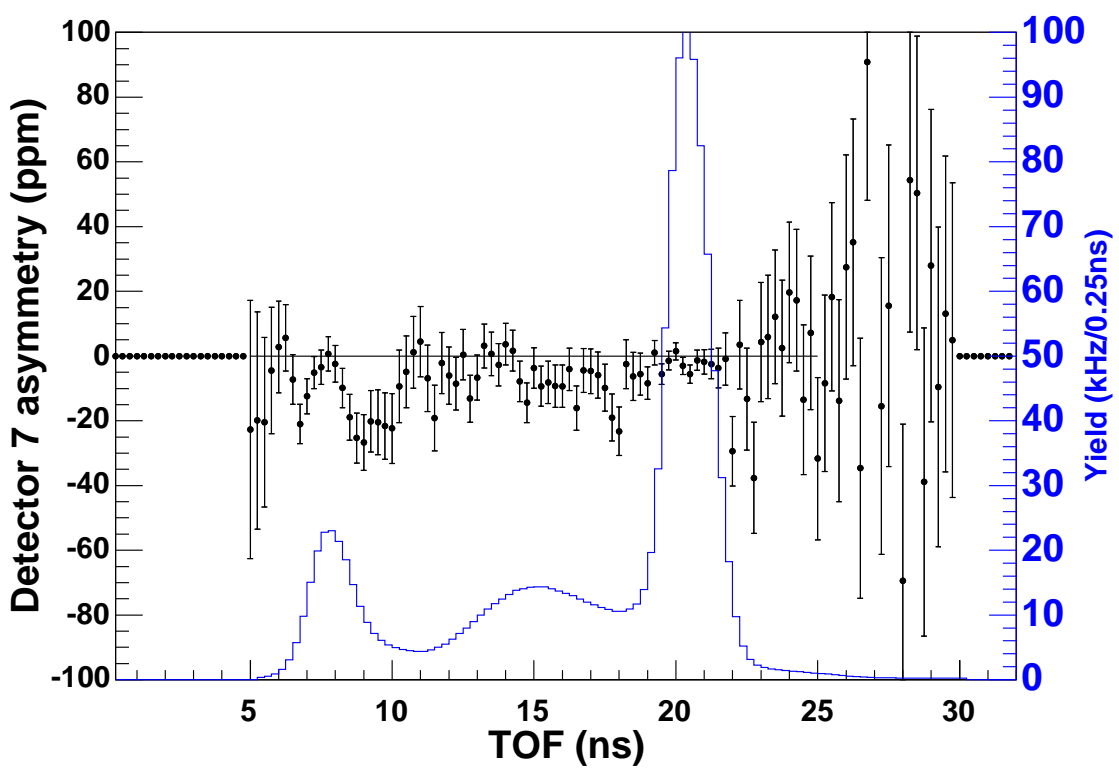

Figure C.7: Octant 2 Detector 7. Asymmetries (black), and yields (blue). 


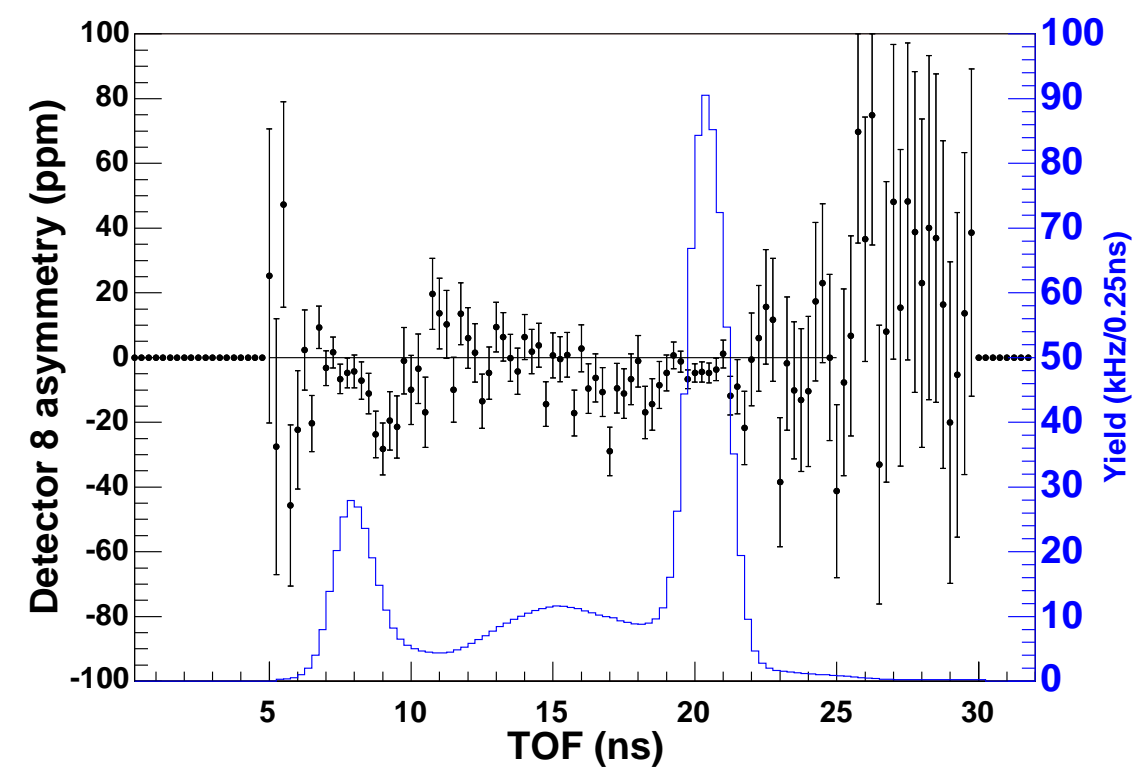

Figure C.8: Octant 2 Detector 8. Asymmetries (black), and yields (blue).

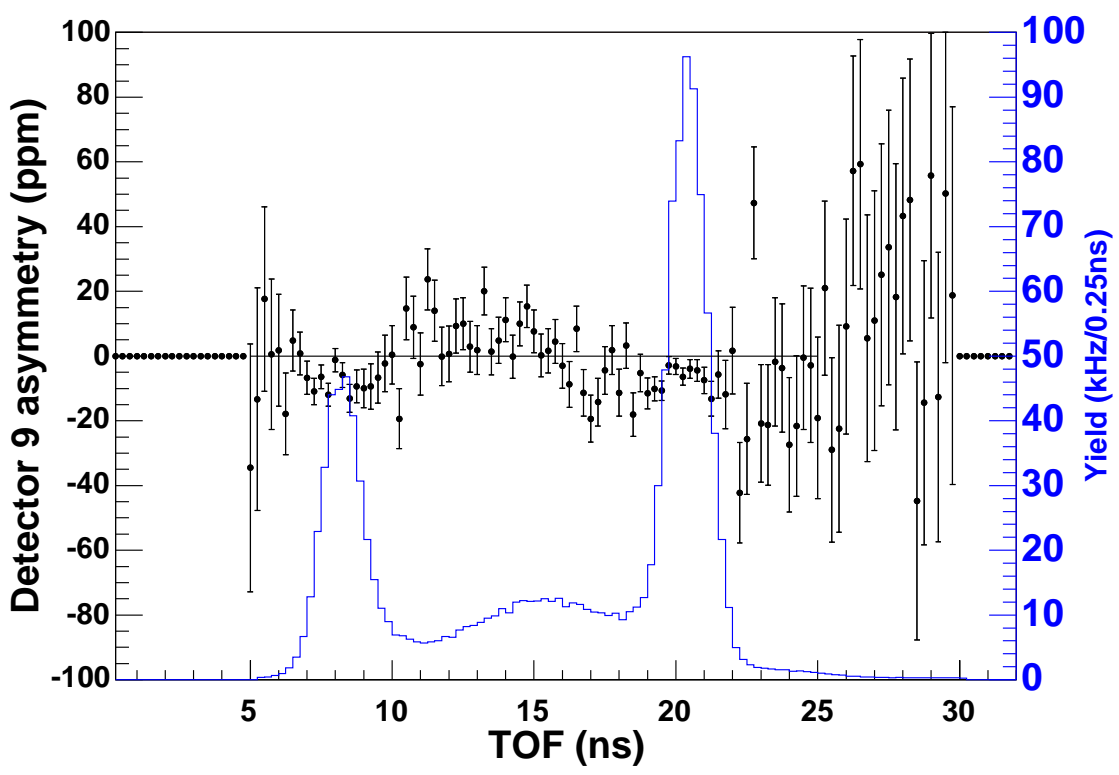

Figure C.9: Octant 2 Detector 9. Asymmetries (black), and yields (blue). 


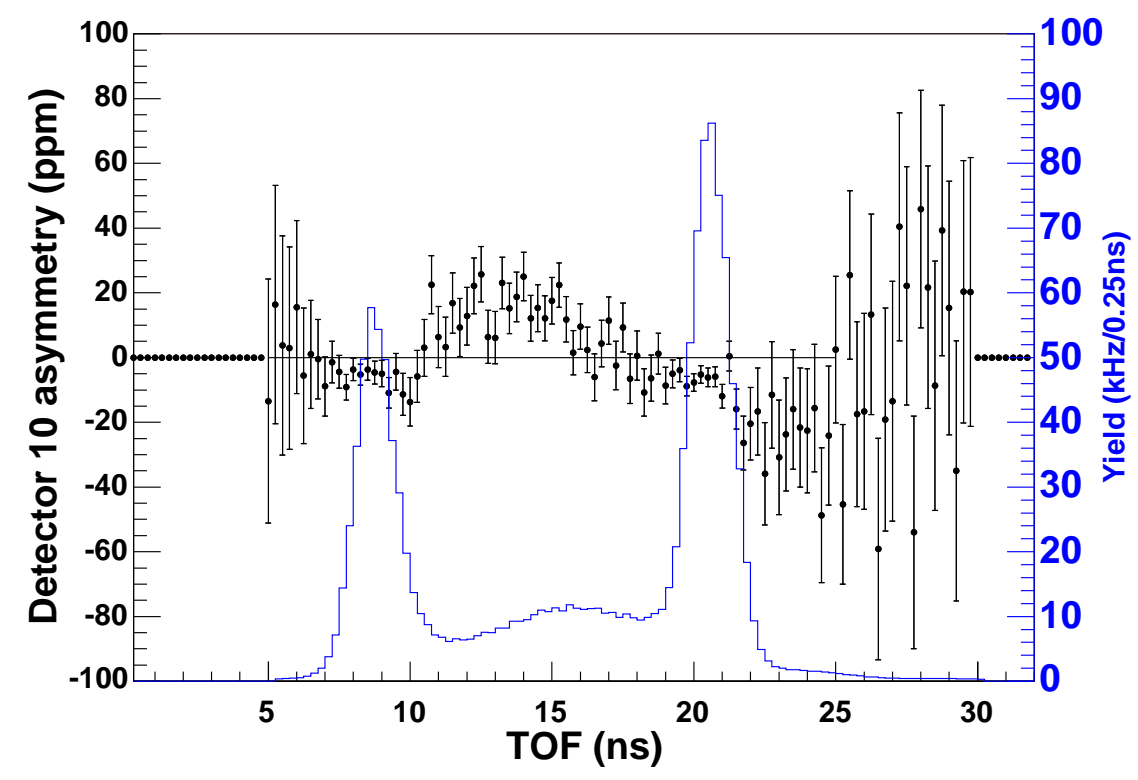

Figure C.10: Octant 2 Detector 10. Asymmetries (black), and yields (blue).

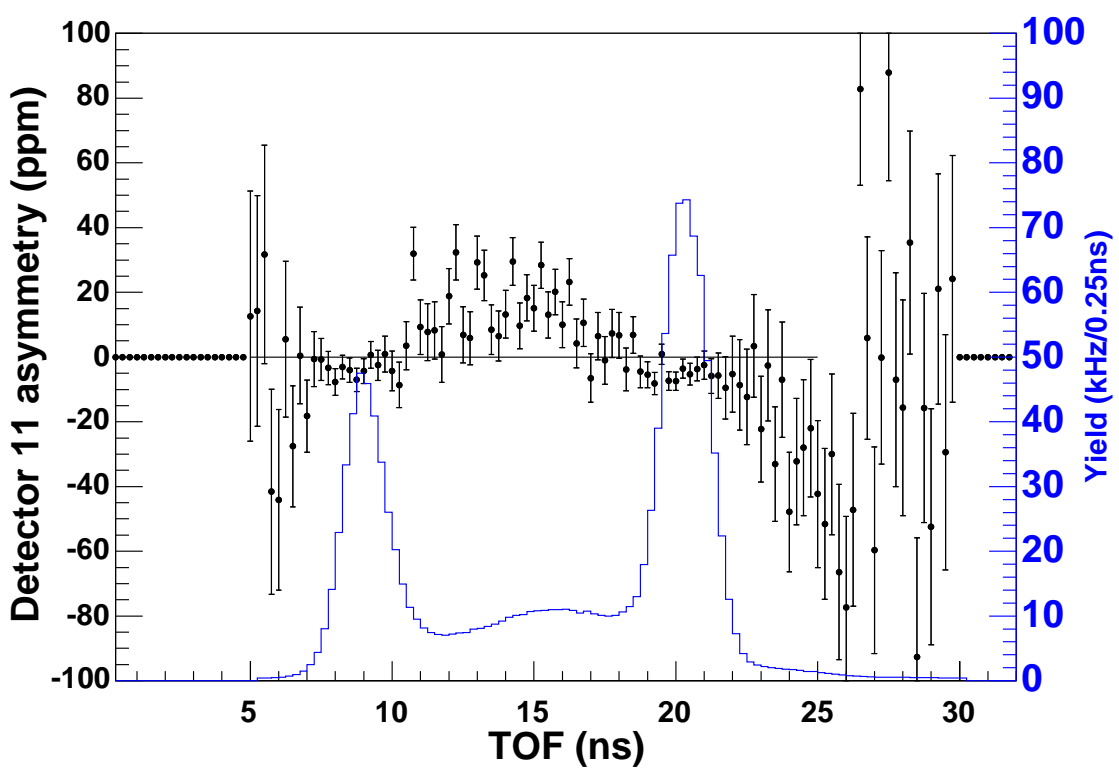

Figure C.11: Octant 2 Detector 11. Asymmetries (black), and yields (blue). 


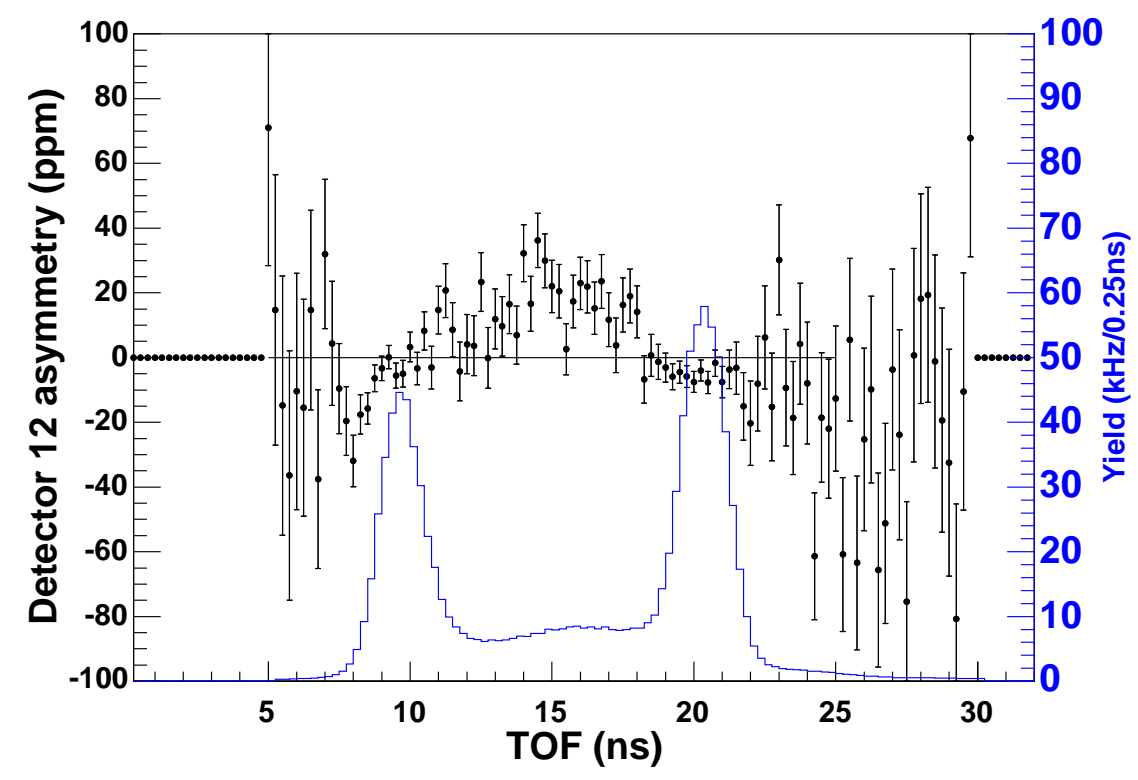

Figure C.12: Octant 2 Detector 12. Asymmetries (black), and yields (blue).

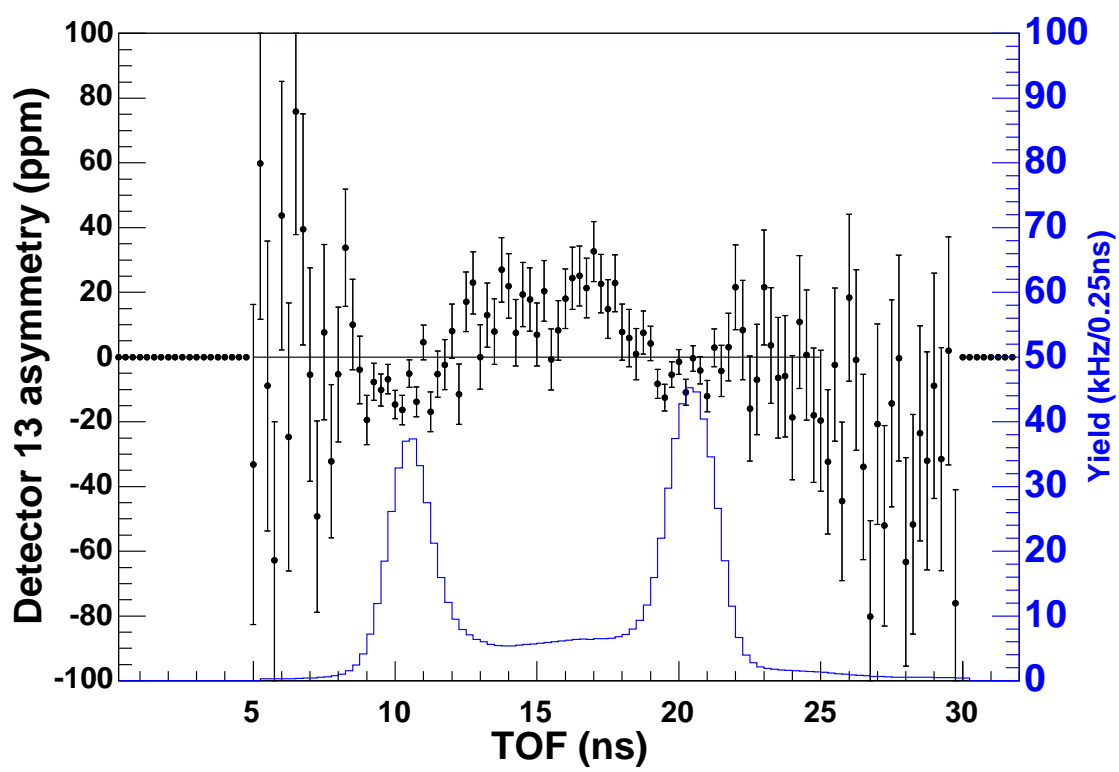

Figure C.13: Octant 2 Detector 13. Asymmetries (black), and yields (blue). 


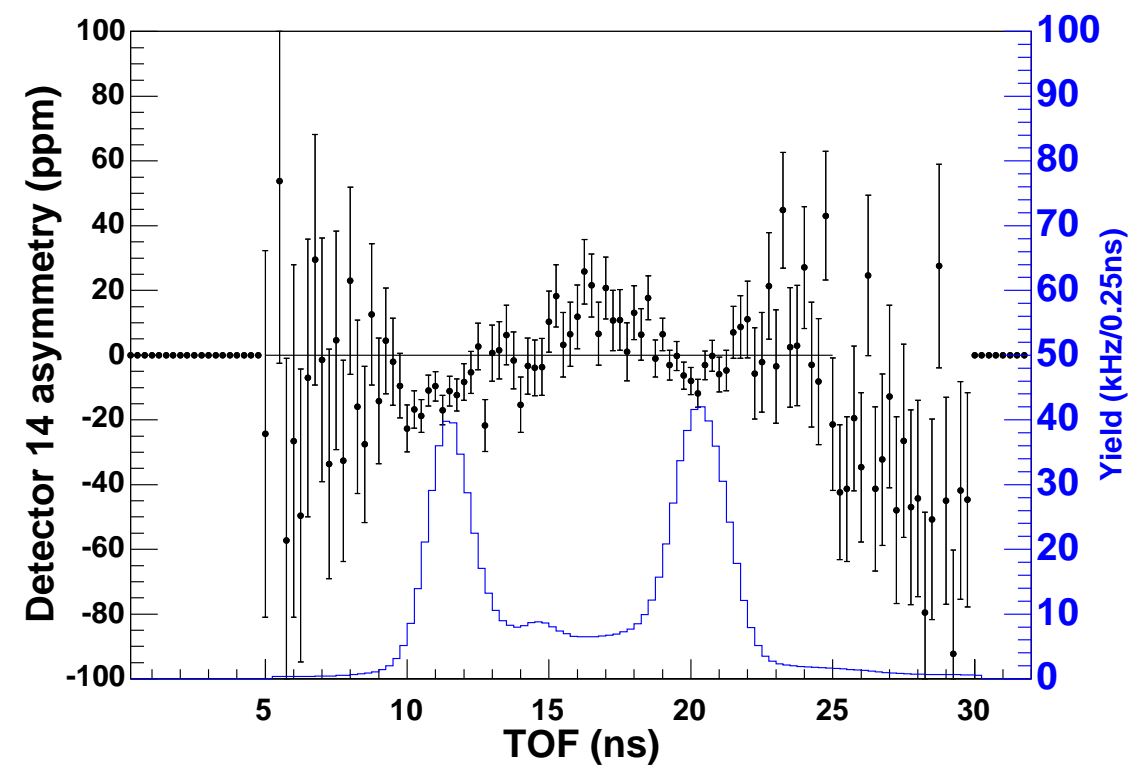

Figure C.14: Octant 2 Detector 14. Asymmetries (black), and yields (blue). The small peak (at around $15 \mathrm{~ns}$ ) immediately after the pion peak is the high- $Q^{2}$ elastic peak, whereas the peak at approximately $20 \mathrm{~ns}$ is the low- $Q^{2}$ elastic peak.

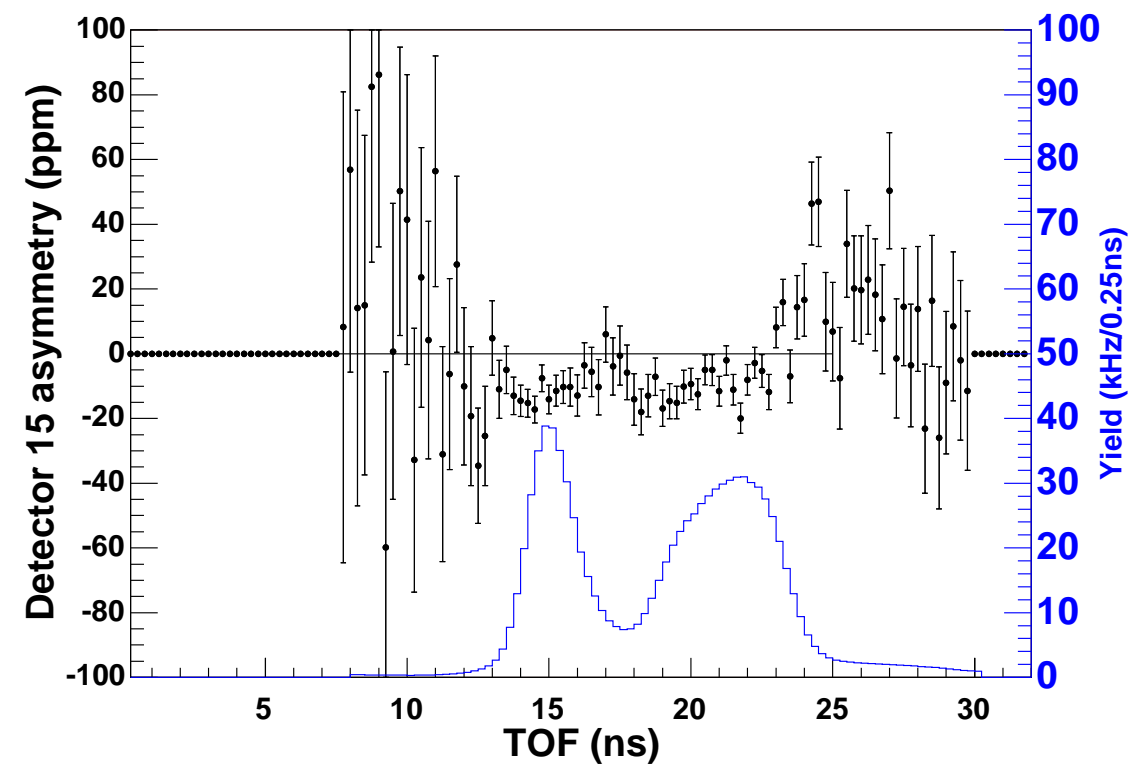

Figure C.15: Octant 2 Detector 15. Asymmetries (black), and yields (blue). The elastic proton peak is a wide distribution due to the fact that this detector is sensitive to a large range of $Q^{2}$. 


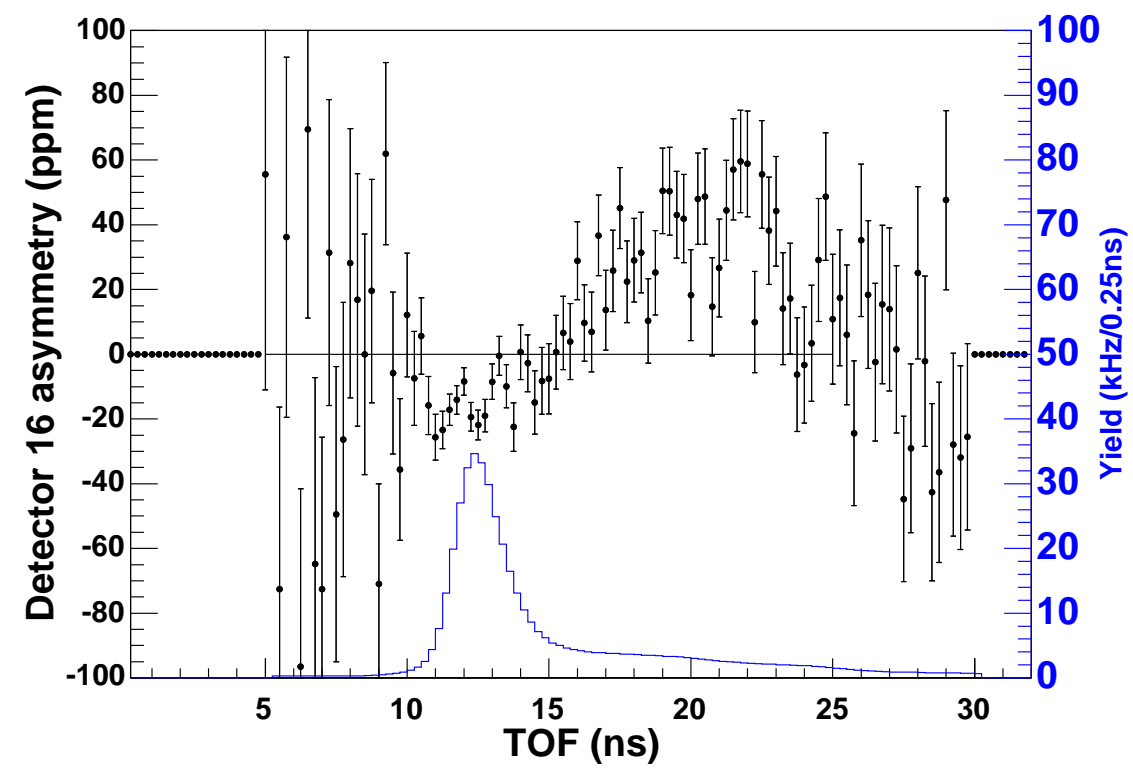

Figure C.16: Octant 2 Detector 16. Asymmetries (black), and yields (blue). 


\section{C.2 Linear Regression Slopes}

\begin{tabular}{|c|c|c|c|c|}
\hline Detector & $\begin{array}{c}\frac{1}{<Y>} \frac{d Y}{d x} \\
(\% / m m) \times 10^{-1}\end{array}$ & $\begin{array}{c}\frac{1}{<Y>} \frac{d Y}{d y} \\
(\% / m m) \times 10^{-2}\end{array}$ & $\begin{array}{c}\frac{1}{<Y>\frac{d Y}{d \theta_{x}}} \\
(\% / m r a d) \times 10^{-1}\end{array}$ & $\begin{array}{c}\frac{1}{<Y>\frac{d Y}{d \theta_{y}}} \\
(\% / m r a d) \times 10^{-1}\end{array}$ \\
\hline 1 & $1.095(0.014)$ & $-2.860(0.139)$ & $-1.536(0.242)$ & $-1.804(0.197)$ \\
\hline 2 & $1.158(0.013)$ & $-3.882(0.125)$ & $1.859(0.219)$ & $-1.453(0.178)$ \\
\hline 3 & $1.201(0.013)$ & $-4.392(0.126)$ & $-2.184(0.220)$ & $-0.642(0.179)$ \\
\hline 4 & $1.318(0.014)$ & $-3.960(0.134)$ & $-2.034(0.233)$ & $-0.887(0.189)$ \\
\hline 5 & $1.326(0.013)$ & $-4.627(0.130)$ & $-1.199(0.227)$ & $-0.488(0.184)$ \\
\hline 6 & $1.417(0.013)$ & $-4.195(0.129)$ & $-7.636(0.226)$ & $-0.376(0.183)$ \\
\hline 7 & $1.486(0.013)$ & $-4.371(0.127)$ & $-7.201(0.221)$ & $-0.391(0.179)$ \\
\hline 8 & $1.620(0.014)$ & $-4.024(0.141)$ & $-10.616(0.245)$ & $-0.306(0.200)$ \\
\hline 9 & $1.565(0.014)$ & $-4.072(0.136)$ & $-12.997(0.238)$ & $-0.606(0.193)$ \\
\hline 10 & $1.505(0.014)$ & $-3.901(0.141)$ & $-15.352(0.245)$ & $-1.138(0.199)$ \\
\hline 11 & $1.527(0.014)$ & $-3.600(0.141)$ & $-13.948(0.247)$ & $-1.202(0.199)$ \\
\hline 12 & $1.439(0.016)$ & $-3.915(0.158)$ & $-13.899(0.274)$ & $-1.563(0.223)$ \\
\hline 13 & $1.496(0.018)$ & $-3.949(0.175)$ & $-11.684(0.306)$ & $-0.858(0.248)$ \\
\hline 14 & $1.404(0.018)$ & $-3.455(0.178)$ & $-12.516(0.315)$ & $-1.215(0.253)$ \\
\hline 15 & $1.280(0.016)$ & $-0.829(0.155)$ & $-25.290(0.270)$ & $-1.003(0.219)$ \\
\hline 16 & $11.294(0.045)$ & $-40.702(0.447)$ & $-102.690(0.782)$ & $21.075(0.634)$ \\
\hline
\end{tabular}

Table C.1: IHWP OUT linear regression slopes for the transverse positions and angles averaged over all octants.

\begin{tabular}{|c|c|c|}
\hline Detector & $\begin{array}{c}\frac{1}{<Y>} \frac{d Y}{d E} \\
(\% / M e V) \times 10^{-2}\end{array}$ & $\begin{array}{c}\frac{1}{<Y>} \frac{d Y}{d I} \\
(\% / n C) \times 10^{-4}\end{array}$ \\
\hline 1 & $2.174(0.058)$ & $-4.502(0.018)$ \\
\hline 2 & $2.265(0.053)$ & $-4.848(0.016)$ \\
\hline 3 & $2.306(0.053)$ & $-3.509(0.016)$ \\
\hline 4 & $2.490(0.056)$ & $-4.376(0.017)$ \\
\hline 5 & $2.550(0.055)$ & $-6.094(0.017)$ \\
\hline 6 & $2.446(0.055)$ & $-5.571(0.017)$ \\
\hline 7 & $2.486(0.053)$ & $-7.439(0.016)$ \\
\hline 8 & $2.460(0.060)$ & $-5.816(0.018)$ \\
\hline 9 & $2.470(0.057)$ & $-8.420(0.018)$ \\
\hline 10 & $2.324(0.059)$ & $-7.405(0.018)$ \\
\hline 11 & $2.193(0.059)$ & $-9.936(0.018)$ \\
\hline 12 & $2.337(0.066)$ & $-3.282(0.020)$ \\
\hline 13 & $1.954(0.074)$ & $-7.978(0.023)$ \\
\hline 14 & $-0.223(0.076)$ & $-7.400(0.023)$ \\
\hline 15 & $15.640(0.065)$ & $-3.274(0.020)$ \\
\hline 16 & $10.624(0.189)$ & $14.698(0.058)$ \\
\hline
\end{tabular}

Table C.2: IHWP OUT linear regression slopes for energy and beam current averaged over all octants. 


\begin{tabular}{|c|c|c|c|c|}
\hline Detector & $\begin{array}{c}\frac{1}{<Y>} \frac{d Y}{d x} \\
(\% / m m) \times 10^{-1}\end{array}$ & $\begin{array}{c}\frac{1}{<Y>} \frac{d Y}{d y} \\
(\% / m m) \times 10^{-2}\end{array}$ & $\begin{array}{c}\frac{1}{<Y>\frac{d Y}{d \theta_{x}}} \\
(\% / m r a d) \times 10^{-1}\end{array}$ & $\begin{array}{c}\frac{1}{<Y>\frac{d Y}{d \theta_{y}}} \\
(\% / m r a d) \times 10^{-1}\end{array}$ \\
\hline 1 & $1.099(0.017)$ & $0.790(0.140)$ & $-6.4496(0.296)$ & $-0.494(0.210)$ \\
\hline 2 & $1.143(0.016)$ & $-0.372(0.126)$ & $-1.817(0.267)$ & $-0.618(0.189)$ \\
\hline 3 & $1.178(0.016)$ & $-0.861(0.127)$ & $-7.241(0.270)$ & $0.625(0.191)$ \\
\hline 4 & $1.314(0.017)$ & $-0.310(0.135)$ & $-6.448(0.285)$ & $0.696(0.202)$ \\
\hline 5 & $1.335(0.016)$ & $-0.972(0.131)$ & $-6.441(0.278)$ & $0.833(0.197)$ \\
\hline 6 & $1.384(0.016)$ & $-1.430(0.130)$ & $-12.493(0.278)$ & $1.574(0.195)$ \\
\hline 7 & $1.505(0.016)$ & $-1.216(0.128)$ & $-14.554(0.271)$ & $1.213(0.192)$ \\
\hline 8 & $1.664(0.017)$ & $-1.486(0.141)$ & $-19.718(0.299)$ & $1.080(0.212)$ \\
\hline 9 & $1.649(0.017)$ & $-1.134(0.138)$ & $-22.485(0.292)$ & $0.836(0.207)$ \\
\hline 10 & $1.545(0.018)$ & $-0.532(0.142)$ & $-24.317(0.302)$ & $0.811(0.213)$ \\
\hline 11 & $1.643(0.018)$ & $-0.656(0.143)$ & $-21.242(0.305)$ & $0.575(0.214)$ \\
\hline 12 & $1.535(0.020)$ & $-0.996(0.158)$ & $-20.132(0.336)$ & $0.059(0.238)$ \\
\hline 13 & $1.596(0.0218)$ & $-1.026(0.176)$ & $-17.425(0.374)$ & $0.172(0.265)$ \\
\hline 14 & $1.666(0.0223)$ & $-0.189(0.179)$ & $-16.453(0.381)$ & $-0.971(0.270)$ \\
\hline 15 & $1.230(0.019)$ & $0.359(0.156)$ & $-29.613(0.330)$ & $0.252(0.234)$ \\
\hline 16 & $11.191(0.055)$ & $-34.911(0.450)$ & $-133.460(0.950)$ & $23.885(0.676)$ \\
\hline
\end{tabular}

Table C.3: IHWP IN linear regression slopes for the transverse positions and angles averaged over all octants.

\begin{tabular}{|c|c|c|}
\hline Detector & $\begin{array}{c}\frac{1}{<Y>} \frac{d Y}{d E} \\
(\% / M e V) \times 10^{-2}\end{array}$ & $\begin{array}{c}\frac{1}{<Y>} \frac{d Y}{d I} \\
(\% / n C) \times 10^{-4}\end{array}$ \\
\hline 1 & $-1.194(0.063)$ & $-4.309(0.018)$ \\
\hline 2 & $-1.329(0.056)$ & $-4.644(0.017)$ \\
\hline 3 & $-0.958(0.057)$ & $-3.363(0.017)$ \\
\hline 4 & $-1.178(0.060)$ & $-4.294(0.018)$ \\
\hline 5 & $-0.969(0.059)$ & $-6.022(0.017)$ \\
\hline 6 & $-0.888(0.059)$ & $-5.299(0.017)$ \\
\hline 7 & $-0.877(0.057)$ & $-7.204(0.017)$ \\
\hline 8 & $-0.892(0.0631)$ & $-5.744(0.018)$ \\
\hline 9 & $-0.682(0.062)$ & $-8.485(0.018)$ \\
\hline 10 & $-0.943(0.065)$ & $-7.652(0.019)$ \\
\hline 11 & $-0.710(0.065)$ & $-9.935(0.019)$ \\
\hline 12 & $-0.611(0.071)$ & $-2.934(0.021)$ \\
\hline 13 & $-1.244(0.079)$ & $-7.887(0.023)$ \\
\hline 14 & $-2.662(0.081)$ & $-7.131(0.024)$ \\
\hline 15 & $13.519(0.070)$ & $-2.578(0.021)$ \\
\hline 16 & $6.791(0.202)$ & $15.729(0.059)$ \\
\hline
\end{tabular}

Table C.4: IHWP IN linear regression slopes for energy and beam current averaged over all octants. 


\section{C.3 Systematics}

Table C.5 shows factors involved in the correction of the measured asymmetry. All except the background factors are either false asymmetries that can be subtracted, or multiplicative factors that simply scale the measured asymmetry.

\begin{tabular}{|c|c|c|c|c|c|c|}
\hline Detector & $\begin{array}{c}\text { Residual } \\
\text { Deadtime }(\mathrm{ppm})\end{array}$ & $\begin{array}{c}\text { Linear } \\
\text { Regression }(\mathrm{ppm})\end{array}$ & $\begin{array}{c}f_{b k g} \\
(\%)\end{array}$ & $\begin{array}{c}A_{b k g} \\
(\mathrm{ppm})\end{array}$ & $\begin{array}{c}\text { Leakage } \\
(\mathrm{ppm})\end{array}$ & $\begin{array}{c}\text { Polarization } \\
(\%)\end{array}$ \\
\hline 1 & 0.095 & -0.017 & 6.1 & -3.69 & -0.71 & 73.7 \\
\hline 2 & 0.061 & -0.019 & 8.4 & -4.36 & -0.71 & 73.7 \\
\hline 3 & -0.007 & -0.018 & 8.5 & -5.49 & -0.71 & 73.7 \\
\hline 4 & 0.014 & -0.021 & 7.7 & -4.05 & -0.71 & 73.7 \\
\hline 5 & -0.041 & -0.026 & 9.6 & -6.13 & -0.71 & 73.7 \\
\hline 6 & -0.027 & -0.024 & 10.0 & -7.94 & -0.71 & 73.7 \\
\hline 7 & -0.034 & -0.028 & 11.0 & -9.76 & -0.71 & 73.7 \\
\hline 8 & -0.027 & -0.024 & 11.0 & -15.39 & -0.71 & 73.7 \\
\hline 9 & -0.007 & -0.029 & 11.6 & -13.53 & -0.71 & 73.7 \\
\hline 10 & -0.007 & -0.026 & 13.6 & -9.73 & -0.71 & 73.7 \\
\hline 11 & -0.007 & -0.032 & 15.4 & -5.35 & -0.71 & 73.7 \\
\hline 12 & 0.054 & -0.013 & 17.4 & 8.33 & -0.71 & 73.7 \\
\hline 13 & 0.027 & -0.027 & 18.2 & 18.37 & -0.71 & 73.7 \\
\hline $14 \mathrm{a}$ & 0.054 & -0.022 & 18.0 & 36.49 & -0.71 & 73.7 \\
\hline $15 \mathrm{a}$ & 0.081 & -0.006 & 19.0 & 40.86 & -0.71 & 73.7 \\
\hline $15 \mathrm{~b}$ & 0.081 & -0.006 & 20.0 & 31.54 & -0.71 & 73.7 \\
\hline $15 \mathrm{c}$ & 0.081 & -0.006 & 40.0 & 15.65 & -0.71 & 73.7 \\
\hline $14 \mathrm{~b}$ & 0.081 & -0.022 & 78.0 & 16.08 & -0.71 & 73.7 \\
\hline
\end{tabular}

Table C.5: Table of correction factors.

Table C.6 shows a summary of systematic uncertainties for the $G^{0}$ forward angle asymmetries. The residual deadtime and linear regression are point to point uncertainties which are independent for each detector. The leakage and polarization corrections are correlated systematic uncertainties, which are uncertainties that can shift the asymmetry of all detector in the same direction. Background corrections involve both types of systematic uncertainties. 


\begin{tabular}{|c|c|c|c|c|c|c|}
\hline FPD & $\begin{array}{c}\text { Residual } \\
\text { Deadtime } \\
(\mathrm{ppm})\end{array}$ & $\begin{array}{c}\text { Linear } \\
\text { Regression } \\
(\mathrm{ppm})\end{array}$ & $\begin{array}{c}\text { Background } \\
(\mathrm{pt}-\mathrm{pt}) \\
(\mathrm{ppm})\end{array}$ & $\begin{array}{c}\text { Background } \\
(\text { correlated }) \\
(\mathrm{ppm})\end{array}$ & Leakage & Polariz. \\
$(\mathrm{ppm})$ & $(\%)$ \\
\hline 1 & 0.118 & $7.2 \times 10^{-3}$ & 0.190 & 0.110 & 0.136 & 0.01 \\
\hline 2 & 0.076 & $7.6 \times 10^{-3}$ & 0.183 & 0.105 & 0.136 & 0.01 \\
\hline 3 & 0.008 & $6.4 \times 10^{-3}$ & 0.173 & 0.100 & 0.136 & 0.01 \\
\hline 4 & 0.017 & $7.6 \times 10^{-3}$ & 0.182 & 0.105 & 0.136 & 0.01 \\
\hline 5 & 0.051 & $9.6 \times 10^{-3}$ & 0.280 & 0.161 & 0.136 & 0.01 \\
\hline 6 & 0.034 & $9.3 \times 10^{-3}$ & 0.323 & 0.186 & 0.136 & 0.01 \\
\hline 7 & 0.042 & $11.0 \times 10^{-3}$ & 0.247 & 0.143 & 0.136 & 0.01 \\
\hline 8 & 0.034 & $10.4 \times 10^{-3}$ & 0.215 & 0.124 & 0.136 & 0.01 \\
\hline 9 & 0.008 & $13.6 \times 10^{-3}$ & 0.258 & 0.149 & 0.136 & 0.01 \\
\hline 10 & 0.008 & $12.6 \times 10^{-3}$ & 0.301 & 0.174 & 0.136 & 0.01 \\
\hline 11 & 0.008 & $11.8 \times 10^{-3}$ & 0.108 & 0.062 & 0.136 & 0.01 \\
\hline 12 & 0.067 & $7.8 \times 10^{-3}$ & 0.527 & 0.304 & 0.136 & 0.01 \\
\hline 13 & 0.034 & $12.8 \times 10^{-3}$ & 0.849 & 0.490 & 0.136 & 0.01 \\
\hline $14 \mathrm{a}$ & 0.067 & $12.0 \times 10^{-3}$ & 0.729 & 0.729 & 0.136 & 0.01 \\
\hline $15 \mathrm{a}$ & 0.101 & $9.9 \times 10^{-3}$ & 1.475 & 1.475 & 0.136 & 0.01 \\
\hline $15 \mathrm{~b}$ & 0.101 & $9.9 \times 10^{-3}$ & 1.273 & 1.273 & 0.136 & 0.01 \\
\hline $15 \mathrm{c}$ & 0.101 & $9.9 \times 10^{-3}$ & 2.554 & 0.350 & 0.136 & 0.01 \\
\hline $14 \mathrm{~b}$ & 0.067 & $12.0 \times 10^{-3}$ & 9.0 & 0 & 0.136 & 0.01 \\
\hline
\end{tabular}

Table C.6: Table of systematic uncertainties. All values have the blinding factor removed. 


\section{References}

[1] Frisch and O.Stern. Z. Phys 855 (1933)

[2] J.Gasser, H.Leutwyler, and M. Saino. PHys. Lett. B253 260 (1991)

[3] J. Ashman et al. Nucl. Phys. B328 (1989)

[4] SLAC-Yale E130, G. Baum et al., Phys. Rev. Lett. 51, 1135 (1983)

[5] D. Mason et al., hep-ex/0405037

[6] D. Kaplan and A. V. Manohar, Phys, Lett. B310, 527 (1988)

[7] R. D. McKeown, Phys. Lett. B219, 140 (1989)

[8] D. H. Beck, Phys. Rev. D39, 3248 (1989)

[9] I. Sick, Phys. Lett. B576, 62-67 (2003)

[10] F. Halzen and A. Martin, Quarks and Leptons: An Introductory Course in Modern Particle Physics, John Wiley \& Sons, New York (1984)

[11] Gerry Miller, Phys. Rev. C57, 1492 (1998)

[12] P. Renton, Electroweak Interactions An Introduction to the Physics of Quarks \& Leptons, Cambridge University Press, Cambridge (1990)

[13] M. J. Musolf et al., Phys. Rep 239, 1 (1994)

[14] Particle Data Group 2004

[15] D. B. Kaplan, and A. Manohar, Nucl. Phys. B310 527 (1988) 
[16] W. J. Marciano and J.L. Rosner, Phys. Rev. Lett. 652963 (1990)

[17] J. Arvieux, Anapole corrections, Internal G0 Report, G0 Analysis Log: Analysis Notes 73

[18] Shi-Lin Zhu, S. J. Puglia, B. R. Holstein, and M. J. Ramsey-Musolf, Phys. Rev. D62, 033008 (2000)

[19] C. S. Wood et al., Science 275, 1759 (1997)

[20] C.M. Maekawa, J.S. Veiga and U. Van Kolck, Phys. Lett. B488 167 (2000)

[21] D. O. Riska, hep-ph/0003132

[22] D. H. Beck and B. R. Holstein, Int. J. Mod. Phys. E10, 1 (2001)

[23] M. J. Musolf and H. Ito, nucl-th/9607021 (1996)

[24] M. Ramsey-Musolf and H. Ito, Phys. Rev. C55 3066 (1997)

[25] M. J. Musolf and M. Burkardt, Z. Phys. C61, 433 (1994)

[26] J. F. Donoghue and B. R. Holstein, Phys. Lett. B436, 331 (1998)

[27] W. Koepf, E. M. Henley, and S. J. Pollock, Phys. Lett. B288 1 (1992)

[28] P. Geiger and N. Isgur, Phys. Rev. D55 299 (1997)

[29] T. Cohen, H. Forkel, and M. Nielsen, Phys. Lett. B 316, 1 (1993)

[30] H. A. Bethe and F. deHoffman, Mesons and Fields (Row and Peterson, Evanston, 1955), Vol II.

[31] S. D. Drell, H. R. Pagels, Phys. Rev. 140, B397-B407 (1965)

[32] R. L. Jaffe, Phys. Lett. B229, 275 (1989)

[33] U.-G. Meissner et al., preprint hep-ph/9701296

[34] H. Weigel, et al., Phys. Lett. B353, 20 (1995)

[35] V. E. Lyubovitskij et al., Phys. Rev. C66, 055204 
[36] S. J. Dong, K. F. Liu, and A. G. Williams, Phys. Rev. D58, 074504 (1998)

[37] R. Lewis, et al., Phys. Rev. D67, 013003 (2003)

[38] D. Leinweber, et al., Phys. Rev. Lett. 94, 212001 (2005)

[39] N. W. Park, J. Schechter, and H. Weigel, Phys. Rev. D43, 869 (1991)

[40] S. Hong and B. Park, Nucl. Phys. A561, 525 (1993)

[41] D. Leinweber, Phys. Rev. D53, 5115 (1996)

[42] M. J. Musolf, H.-W. Hammer, and D. Drechsel, Phys. Rev. D55 2741 (1997)

[43] D. T. Spayde et al., Phys. Lett. B 583, 79 (2004)

[44] T. M. Ito et al., Phys. Rev. Lett. 92102003 (2004)

[45] K. A. Aniol et al., Phys. Rev. C 69, 065501 (2004)

[46] K. A. Aniol et al., nucl-ex/0506010 (2005)

[47] K. A. Aniol et al., nucl-ex/0506011 (2005)

[48] F. E. Maas et al., Phys. Rev. Lett. 93022002 (2004)

[49] F. E. Maas et al., nucl-ex/0412030 (2004)

[50] D. S. Armstrong et al., Phys. Rev. Lett. 95092001 (2005)

[51] http://www.npl.uiuc.edu/exp/G0

[52] http://www.jlab.org

[53] M. Poelker and J. Grames, DOE Science and Technology Review, August 31, 2005

[54] Bandwidth Semiconductor, LLC 25 Sagamore Park Drive, Hudson, NH 03051 (http://www.bandwidthsemi.com)

[55] Time-Bandwidth Products, Inc. Halle Zeppelin 1.0G Technoparkstrasse 1 CH-8005 Zurich (http://www.tbwp.com) 
[56] Thorlabs, Inc. 435 Route 206 North, Newton, NJ 07860 (http://www.thorlabs.com)

[57] J. Grames, Presented at JLab Graduate Student Seminar (2004)

[58] M. Poelker and J. Hansknecht, A High Power and High Repetition Rate Modelocked TISapphire Laser for Photoinjectors PAC paper (2001)

[59] F. L. Pedrotti and L. S. Pedrotti Introduction to Optics Prentice Hall, New Jersey, 1993

[60] P. Saez et al., Polarization studies of strained GaAs photocathodes at the SLAC Gun Test Laboratory

[61] T. Maruyama et al., Phys. Rev. B 46, 4261 (1992)

[62] C. Hyde-Write et al. Beam Position Studies for E93050 1999

[63] R.Kazimi et al., CEBAF Injector Achieved World's Best Beam Quality for Three Simultaneous Beams with a Wide Range of Bunch Charges, Proceedings of EPAC (2004)

[64] C. Møller. Ann. Phys. 14531 (1932)

[65] S.D. Covrig et al., nucl-ex/0502019 (2005)

[66] Barber-Nichols Inc. 6325 West 55th Avenue, Arvada, CO 80002 (http://www.barbernichols.com)

[67] Steven Williamson, Andy Kenyon, Kazutaka Nakahara, and Damon Spayde, G0 Superconducting Magnet System: Operational Safety Procedure (2002)

[68] Dynapower Co. 85 Meadowland Drive, South Burlington, VT 05403 (http://www.dynapower.com)

[69] MKS Inc. 410 Albert Street, Waterloo, ON Canada N2L 3V3 (http://www.mks.com)

[70] Proteus Industries, 340 Pioneer Way, Mountain View, CA 94041 (http://www.proteusind.com)

[71] Automationdirect.com, 3505 Hutchinson Road, Cumming, GA 30040 (http://www.automationdirect.com) 
[72] Lake Shore Cryotronics, Inc. 575 McCorkle Blvd, Westerville, $\quad$ OH 43082 (http://www.lakeshore.com)

[73] Philips, Groenewoudseweg 15621 BA, Eindhoven Netherlands

[74] Photonis, Dwazziewegen 2, 9301 ZR Roden, PO Box 60, 9300 AB Roden, Netherlands

[75] D. Marchand and P. King, Electronics and Data Acquisition for $G^{0}$ Forward-angle Measurements, Proceedings to the PAVI/2002 Conference (2002)

[76] R. Clark and B. Quinn, Internal G0 Report G0-98-010

[77] http://root.cern.ch

[78] http://www.mysql.com

[79] P.R. Bevington and D.K. Robinson, Data Reduction and Error Analysis for the Physical Sciences, WCB McGraw-Hill, Massachusetts, 1992

[80] L. Hannelius, Internal G0 Report G0-03-102

[81] L. Hannelius, Internal G0 Report G0-04-020

[82] B. Guillon, Internal G0 Report G0-04-062

[83] J. W. Lightbody and J. S. O’Connell, Computers in Physics, May/June (1988) 57-64

[84] J. Liu, $G^{0}$ Leakage Correction, Internal G0 Report, G0 Analysis Log: Analysis Notes 110

[85] D. Carman et al., Phys. Rev. Let. 90, 131804 (2003)

[86] J. Liu, $G^{0}$ Hyperon Simulation, Internal G0 Report, G0 Analysis Log: Analysis Notes 111

[87] J. Liu, Ph.D. thesis, University of Maryland (2006)

[88] D. Carman et al., Phys. Rev. Lett. 90, 131804 (2003)

[89] P. King and S. P. Wells et al., Measurement of the vector analyzing power in e-p scattering using the $G^{0}$ forwrd angle apparatus, G0 Document 437-v1 (2005) 
[90] Jacques Arvieux et al., Calculation of electromagnetic radiative corrections for $G^{0}$, Internal G0 Report, G0 Analysis Log: Analysis Notes 75

[91] S. Phillips, Beam Polarization Numbers, Internal G0 Report, G0 Analysis Log: Analysis Notes 84

[92] J. J. Kelly, Phys. Rev. C 70, 068202 (2004)

[93] J. Arrington, Phys. Rev. C 69, 022201 (2004)

[94] J. Friedrich and T. Walcher, Eur. Phys. J. A 17, 607 (2003) 


\section{Author's Biography}

Kazutaka Nakahara was born in Japan, but moved to the United States soon after birth. He attended Ridgewood High School, after which he enrolled and studied physics at Carnegie-Mellon University. After receiving his B.S in physics, he proceeded to attend the University of Illinois at Urbana-Champaign to study nuclear physics.

\section{Publications}

Strange Quark Contribution to Parity-Violating Asymmetries in the Forward G0 Electron Proton Scattering Experiment, The G0 Collaboration, D. S. Armstrong et at., Phys. Rev. Lett. 95, $092001(2005)$

G0 Beam Quality and Multiple Linear Regression Corrections, proceedings to presentation at PAVI'04 